UNIVERSIDADE DE SÃO PAULO

INSTITUTO DE FÍSICA

\title{
Grupo de Renormalização na Aproximação de Potencial Local para o Modelo $O(N)$ de Heisenberg Hierárquico: Trajetória Crítica e Somabilidade da Expansão $1 / N$
}

\section{William Remo Pedroso Conti}

Orientador: Prof. Dr. Domingos Humberto Urbano Marchetti

Tese de Doutorado
apresentada ao Instituto de Física
da Universidade de São Paulo
para a obtenção do título de
Doutor em Ciências.

Banca Examinadora:

Prof. Dr. Carlos Eugênio Imbassahy Carneiro - IFUSP

Prof. Dr. Gastão de Almeida Braga - UFMG

Prof. Dr. João Carlos Alves Barata - IFUSP

Prof. Dr. Paulo Domingos Cordaro - IMEUSP

Prof. Dr. Silvio Roberto de Azevedo Salinas (presidente) - IFUSP

São Paulo 



\section{Agradecimentos}

À minha amada família - Sra. Maria Cristina Pedroso Conti, Sr. Leonildo Remo Conti, Thiago Vinícius Pedroso Conti e Mayara Cristina Pedroso Conti - pelo apoio e incentivo.

Ao meu orientador, Prof. Dr. Domingos Humberto Urbano Marchetti, pelo modo como conduziu meus passos; por toda sua atenção, em todos os momentos deste projeto; pela confiança em mim depositada.

Ao Prof. Dr. Silvio Roberto de Azevedo Salinas, que aceitou ficar por mim responsável nos meus últimos meses de doutorado, por conta da viagem de meu orientador.

Aos meus amigos do Grupo de Mecânica Estatística, meus companheiros de caminhada. Às secretárias e funcionários do Departamento de Física Geral, pelo suporte e prestividade.

À Fundação de Amparo à Pesquisa do Estado de São Paulo (FAPESP), pelo apoio financeiro. 



\section{Resumo}

$\mathrm{Na}$ aproximação de potencial local $(L \downarrow 1)$ a transformação de grupo de renormalização para o modelo $O(N)$ de Heisenberg hierárquico na criticalidade (sistema à temperatura inversa crítica $\left.\beta_{c}(N, d)\right)$ é descrita pela equação

$$
u_{t}^{(N)}=\frac{2}{N} x u_{x x}^{(N)}+u_{x}^{(N)}-2 x\left(u_{x}^{(N)}\right)^{2}-(d+2) x u_{x}^{(N)}+d u^{(N)}-u_{x}^{(N)}(t, 0),
$$

sendo que a condição inicial definida pelo modelo é dada por

$$
u^{(N)}(0, x)=-\frac{1}{N} \ln \left[\frac{\Gamma(N / 2)}{(i \sqrt{\beta x} N / 2)^{N / 2-1}} J_{N / 2-1}(i \sqrt{\beta x} N)\right] .
$$

O objetivo deste trabalho é investigar a somabilidade da série formal em potências de $1 / N$ da equação de evolução satisfeita por $u_{x}^{(N)}(t, x)$ quando o sistema encontra-se na criticalidade $\left(\beta=\beta_{c}(N, d)\right)$. Considerando a função inversa $v^{(N)}(t, p)=\left(u_{x}^{(N)}\right)^{-1}(t, p)$, mostramos que na criticalidade a série formal

$$
\hat{v}^{(N)}(t, p)=\sum_{i=0}^{\infty} v^{[i]}(t, p)(2 / N)^{i}
$$

é $s$-Gevrey, para todo $p$ fixo pertencente ao disco fechado centrado em $p=-1$ e raio $\varsigma$ (denotado por $\left.\overline{D_{\varsigma}(-1)}\right)$, com $s>2$ e $0<\varsigma<1 / 2$. Mostramos também que a série formal

$$
\hat{u}_{x}^{(N)}(0, x)=\sum_{i=0}^{\infty} f_{i}(x)(2 / N)^{i}
$$

é 1-somável na direção $\theta=0$. 



\section{Abstract}

In the local potential approximation $(L \downarrow 1)$ the renormalization group transformation for the hierarchical $O(N)$ Heisenberg model at criticality (system at inverse critical temperature $\left.\beta_{c}(N, d)\right)$ is given by the evolution equation

$$
u_{t}^{(N)}=\frac{2}{N} x u_{x x}^{(N)}+u_{x}^{(N)}-2 x\left(u_{x}^{(N)}\right)^{2}-(d+2) x u_{x}^{(N)}+d u^{(N)}-u_{x}^{(N)}(t, 0),
$$

and the initial condition defined by the model is given by

$$
u^{(N)}(0, x)=-\frac{1}{N} \ln \left[\frac{\Gamma(N / 2)}{(i \sqrt{\beta x} N / 2)^{N / 2-1}} J_{N / 2-1}(i \sqrt{\beta x} N)\right] .
$$

The main goal of this work is to investigate the summability of the formal power series (in $1 / N)$ solution of the evolution equation satisfied by $u_{x}^{(N)}(t, x)$ when the system is at criticality $\left(\beta=\beta_{c}(N, d)\right)$. Considering the inverse function $v^{(N)}(t, p)=\left(u_{x}^{(N)}\right)^{-1}(t, p)$, we show that at criticality the formal series

$$
\hat{v}^{(N)}(t, p)=\sum_{i=0}^{\infty} v^{[i]}(t, p)(2 / N)^{i}
$$

is $s$-Gevrey, for every fixed $p$ in the closed disc centered at $p=-1$ and radius $\varsigma$ (denoted by $\left.\overline{D_{\varsigma}(-1)}\right)$, where $s>2$ and $0<\varsigma<1 / 2$. We also show that the formal series

$$
\hat{u}_{x}^{(N)}(0, x)=\sum_{i=0}^{\infty} f_{i}(x)(2 / N)^{i}
$$

is 1-summable in $\theta=0$ direction. 



\section{Sumário}

1 Motivações e Resultados 12

2 Trajetória Discreta: Resumo 31

2.1 O Modelo $O(N)$ de Heisenberg Hierárquico . . . . . . . . . . . . . . . . . 31

2.2 Transformação de Grupo de Renormalização . . . . . . . . . . . . . . . . 39

2.3 A Aproximação de Potencial Local $(L \downarrow 1)$. . . . . . . . . . . . . . . . 58

3 Definições e Ferramentas $\quad 64$

3.1 Teoria de Somabilidade: Definições . . . . . . . . . . . . . . . . 64

3.2 Normas e Estimativas . . . . . . . . . . . . . . . . . 67

3.3 Ingredientes Chave . . . . . . . . . . . . . . . . . . 72

3.4 Propriedades de $g(\varepsilon, w)$ a Partir das Propriedades de $f(\varepsilon, z)=g^{-1}(\varepsilon, z)$. 79

4 Estudo da Condição Inicial $\quad 88$

4.1 A Classe $P$ das Funções de Pick . . . . . . . . . . . . . . . . . . . 88

4.2 Raio de Univalência . . . . . . . . . . . . . . . . . . . . 96

4.3 1-Somabilidade na Direção $\theta=0$. . . . . . . . . . . . . . 102

4.3.1 A EDO Satisfeita por $u(\varepsilon, 0, x)=u^{(N)}(0, x) \ldots \ldots$. . . . . . 103

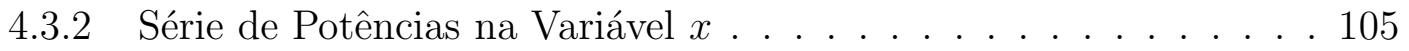

4.3.3 Série de Potências na Variável $\varepsilon=2 / N$. . . . . . . . . . . 108

4.3.4 Série Assintótica . . . . . . . . . . . . . . . . . . . 112

4.3.5 Concluindo a Seção: o Teorema . . . . . . . . . . . . . . . 118

5 O Caso $t>0$ na Criticalidade $\quad 120$

5.1 Transformada de Legendre de $u(\varepsilon, t, x)=u^{(N)}(t, x)$ com Respeito a $x$. . 120

5.2 Série de Potências na Variável $\varepsilon=2 / N \ldots$. . . . . . . . . . . 123 
5.2.1 Equação Satisfeita por Cada Coeficiente $v^{[i]}(t, p)$. . . . . . . . . 124

5.2 .2 A Criticalidade . . . . . . . . . . . . . . . . 129

5.2.3 Existência de uma Única Solução $v^{[i]}(t, p)$ na Criticalidade . . . . 130

5.2.4 Estimativas de Gevrey para $\left|v^{[i]}(t, p)\right|$ na Criticalidade . . . . . . 139

5.3 Série Assintótica . . . . . . . . . . . . . . . . . . . . . . . . 148

6 Conclusões e Comentários Finais $\quad 149$

A Apêndices 152

A.1 A Classe $P$ das Funções de Pick . . . . . . . . . . . . . . . . . . . . . 152

A.2 Frações Continuadas e o Teorema de Worpitzky . . . . . . . . . . . . 166 



\section{Capítulo 1}

\section{Motivações e Resultados}

A aproximação de potencial local é uma versão de escala contínua para uma transformação de grupo de renormalização. Como dito por Zumbach em [51], essa é a mais simples "boa aproximação" do grupo de renormalização, entendendo por "boa aproximação" aquela que é suficientemente simples para que possamos lidar com ela, e suficientemente sofisticada para que contenha a essência física do problema.

No ano de 1987, em um trabalho seminal, Felder [17] estudou a versão de escala contínua do modelo hierárquico de Dyson [14]. Ela é dada pela equação diferencial parcial

$$
\psi_{t}=\frac{1}{2} \psi_{y y}-\frac{1}{2}\left(\psi_{y}\right)^{2}-\frac{d-2}{2} y \psi_{y}+d \psi,
$$

que descreve o fluxo do potencial efetivo $\psi(t, y)(\psi(t, y)=-\ln \sigma(t, y)$, com $\sigma(t, y)$ uma medida de probabilidade) na escala $e^{-t}$ como função do campo (variável de spin) $y \in \mathbb{R}$, sendo que $d>2$. Tal equação pode ser derivada de duas formas, sendo que uma delas é pelo limite de passos infinitesimais $(L \downarrow 1)$ da relação de recorrência (transformação de grupo de renormalização) do modelo hierárquico, sendo $L$ um parâmetro associado ao tamanho dos blocos de spin - Felder considera o modelo hierárquico de Dyson na versão introduzida por Gallavotti [22] e mostra que com o limite $L \downarrow 1$ a relação de recorrência torna-se (1.0.1). Conforme observado por Felder, é possível impor uma condição de normalização à $\psi(t, y)$, por exemplo, $\psi(t, 0)=0$ para todo $t \geq 0$; para tanto, basta que introduzamos um multiplicador de Lagrange $\theta(t)$ na equação (1.0.1). 
Na aproximação de potencial local os pontos fixos da transformação de grupo de renormalização devem ser identificados com soluções globalmente estacionárias de (1.0.1). Assim, o problema de encontrar pontos fixos na aproximação de potencial local se reduz ao estudo de soluções globais (definidas para todo $y$ ) da seguinte equação diferencial ordinária:

$$
\psi^{\prime \prime}-\left(\psi^{\prime}\right)^{2}-(d-2) y \psi^{\prime}+2 d \psi=0
$$

O que Felder mostra é que, além do ponto fixo gaussiano (também chamado de ponto fixo trivial) $\psi^{\text {gauss }}(y)=0$ e do ponto fixo de altas temperaturas $\psi^{a t}(y)=y^{2}-1 / d$, a equação (1.0.1) possui soluções globalmente estacionárias não-triviais $\psi_{2 n}^{*}(y)$ com a forma de $n$ potenciais do "tipo poço" para $2<d<d_{n}=2+2 /(n-1), n=2,3, \ldots$ A estabilidade local dos pontos fixos triviais e algumas propriedades dinâmicas da equação de evolução correspondente ao sistema de spins de $N$-componentes foram estudadas analítica e numericamente por Zumbach [51]. Em [37], Lima refinou o estudo de Felder para o domínio de dimensões $d \geq 3$. Após isso, pouca atenção foi dada à equação (1.0.1), sendo que um dos principais obstáculos aos avanços está no fato de o espaço de configuração $\mathbb{R}^{N} \times \cdots \times \mathbb{R}^{N}=\mathbb{R}^{N . n}$ de $n$ spins ser não-compacto.

No ano de 2005 iniciamos nossos estudos sobre o modelo $O(N)$ de Heisenberg hierárquico. Inicialmente, como parte de um estudo preliminar ao projeto de mestrado, estendemos e complementamos para esse modelo os resultados obtidos por Kunz e Zumbach [34]: mostramos em [8] a convergência da energia livre e da função geratriz do modelo $O(N)$ de Heisenberg hierárquico para as do modelo esférico hierárquico quando os limites $n \rightarrow \infty$ (limite termodinâmico) e $N \rightarrow \infty$ são tomados, independentemente da ordem de tais limites. Para tanto, utilizamos uma adaptação do método empregado por Kac e Thompson [28], além do já citado artigo [34] de Kunz e Zumbach, e uma generalização $(L>1$ e $d \geq 1)$ da definição de positividade por reflexão para o laplaceano hierárquico encontrada em [49]. Devemos destacar que tais resultados valem para qualquer $\beta<\beta_{c}$; eles não incluem a criticalidade $\left(\beta=\beta_{c}\right)$. Além da extensão acima apontada, mostramos em [8] que o modelo esférico hierárquico exibe transição de fase para $d>2$.

A idéia de realizar esse estudo preliminar era a de que ele servisse como guia para a investigação dos modelos $O(N)$ de Heisenberg hierárquico e esférico hierárquico na 
criticalidade, o que requer a implementação do grupo de renormalização. Visando um entendimento mais claro e profundo de algumas questões, optamos por trabalhar com o grupo de renormalização na aproximação de potencial local. O estudo do modelo esférico hierárquico na criticalidade foi feito entre os anos de 2006 e 2008, resultando no artigo [41] e na dissertação de mestrado [7]. O presente projeto de pesquisa dá continuidade às investigações realizadas no mestrado, sendo o modelo $O(N)$ de Heisenberg hierárquico na criticalidade o nosso foco. Nossos estudos têm contribuído em algumas direções, que serão explicitadas ao longo deste capítulo tão logo o contexto em que aparecem e os conceitos envolvidos sejam introduzidos.

O Modelo $O(N)$ de Heisenberg Hierárquico. Dados os inteiros $L, K$ e $d$, com $L, K>1$ e $d \geq 1$, seja

$$
\Lambda_{K}=\left\{0,1, \ldots, L^{K}-1\right\}^{d} \subset \mathbb{Z}^{d}
$$

uma rede finita hipercúbica $d$-dimensional de cardinalidade $\left|\Lambda_{K}\right|=L^{K d}=n$. Por modelo $O(N)$ de Heisenberg hierárquico entende-se o modelo que associa a cada vértice de $\Lambda_{K}$ uma variável de spin clássica y que assume valores sobre a esfera de raio $\sqrt{\beta N}$ em $\mathbb{R}^{N}$, $\operatorname{com} \beta$ o inverso da temperatura. Essas variáveis são dependentes segundo uma matriz de acoplamento ferromagnética chamada matriz de interação hierárquica $\left(J_{H}\right)$. Tratase de uma matriz $n \times n$ real simétrica, positiva e positiva definida, que não obedece invariância por translação nem alcance finito, e cuja forma quadrática é dada por

$$
\begin{aligned}
\left(s, J_{H} s\right)_{\mathbb{R}^{n}} & =\sum_{k=1}^{K} L^{-2 k} \sum_{\boldsymbol{r} \in \Lambda_{K-k}}\left(B^{k} s\right)_{\boldsymbol{r}}^{2} \\
\left(B^{k} s\right)_{\boldsymbol{r}} & =\frac{1}{L^{d k / 2}} \sum_{\boldsymbol{j} \in \Lambda_{k}} s_{L^{k}} \boldsymbol{r}+\boldsymbol{j}
\end{aligned}
$$

$\operatorname{com}(r, s)_{\mathbb{R}^{n}}=\sum_{\boldsymbol{i} \in \Lambda_{K}} r_{\boldsymbol{i}} s_{\boldsymbol{i}}$. Vê-se assim que as variáveis de spin interagem segundo uma estrutura de blocos: a cada hierarquia são formados blocos de $L^{d}$ variáveis de spin da hierarquia anterior, de tal maneira que na hierarquia $k$ tem-se a rede $\Lambda_{K-k}$ de cardinalidade $L^{(K-k) d}$ (vide Figura 2.1 na página 36 - exemplo em que $L=2$ e $d=1$ ). 
Posto isso, temos que o modelo $O(N)$ de Heisenberg hierárquico é definido pela medida de equilíbrio (medida de Gibbs) $O(N)$ invariante

$$
d \nu_{n}^{(N)}(\boldsymbol{y})=\frac{1}{Z_{n}^{(N)}} \exp \left\{-\frac{1}{2} \mathcal{U}_{n}(\boldsymbol{y})\right\} \prod_{\boldsymbol{i} \in \Lambda_{K}} d \sigma_{0}^{(N)}\left(y_{\boldsymbol{i}}\right)
$$

sendo que $\boldsymbol{y}$ denota um elemento do espaço de configuração $\Omega_{n}=\mathbb{R}^{N} \times \cdots \times \mathbb{R}^{N}=\mathbb{R}^{N . n}$;

$$
\mathcal{U}_{n}(\boldsymbol{y})=-(L-1)\left(\boldsymbol{y},\left(J_{H} \otimes I_{N}\right) \boldsymbol{y}\right)_{\Omega_{n}}
$$

$\operatorname{com}(\boldsymbol{x}, \boldsymbol{y})_{\Omega_{n}}=\sum_{\boldsymbol{i} \in \Lambda_{K}} x_{\boldsymbol{i}} \cdot y_{\boldsymbol{i}}=\sum_{\boldsymbol{i} \in \Lambda_{K}} \sum_{q=1}^{N} x_{\boldsymbol{i}}^{(q)} y_{\boldsymbol{i}}^{(q)}$ e $I_{N}$ a matriz identidade de ordem $N$

$$
d \sigma_{0}^{(N)}(y)=\frac{1}{S_{\beta N}^{N}} \delta(|y|-\sqrt{\beta N}) d^{N} y
$$

é a medida "a priori" uniforme sobre a esfera $N$-dimensional de raio $\sqrt{\beta N}$ e $S_{\beta N}^{N}$ a área da superfície dessa esfera.

Transformação de Grupo de Renormalização. Seja $\sigma_{k}^{(N)}(y)$ a distribuição "a priori" da variável soma

$$
X_{k}^{(N)}=\frac{1}{\sqrt{m^{1+2 / d}}} \sum_{i \in \Lambda_{k}} y_{i}
$$

com $m=\left|\Lambda_{k}\right|=L^{k d}$. Se $\mathcal{M}$ denota o espaço das distribuições "a priori" (medidas de probabilidade) em $\mathbb{R}^{N}$ invariantes por transformações ortogonais $O(N)$, o modelo hierárquico permite estabelecer, em razão da estrutura auto-similar da energia $\mathcal{U}_{n}(\boldsymbol{y})$, um mapa discreto $\mathcal{R}: \mathcal{M} \rightarrow \mathcal{M}$ (vide Proposição 2.2.1):

$$
\sigma_{k}^{(N)}(y)=\frac{1}{C_{k}} e^{(L-1)|y|^{2} / 2} \underbrace{\sigma_{k-1}^{(N)} * \cdots * \sigma_{k-1}^{(N)}}_{L^{d}-\text { termos }}\left(L^{1+d / 2} y\right)
$$

sendo que $*$ denota o produto de convolução 


$$
\rho * \eta(y)=\int_{\mathbb{R}^{N}} \rho\left(y-y^{\prime}\right) d \eta\left(y^{\prime}\right)
$$

e $C_{k}$ uma normalização que garante que $\sigma_{k}^{(N)}$ é uma medida de probabilidade. A convolução de $L^{d}$ medidas "a priori" da hierarquia anterior decorre do fato que

$$
X_{k}^{(N)}=L^{-(1+d / 2)}(\underbrace{X_{k-1}^{(N)}+\ldots+X_{k-1}^{(N)}}_{L^{d}-\text { termos }})
$$

lembrando que $\sigma_{k-1}^{(N)}(y)$ é a distribuição da variável $X_{k-1}^{(N)}$; o termo $e^{(L-1)|y|^{2} / 2}$ vem da energia (termo de interação), e portanto traz a informação de que as variáveis de spin são dependentes (vide Observação 2.2.2).

Sendo $\left\{\sigma_{k}^{(N)}(y ; \beta)\right\}_{k=0}^{\infty}$ uma família de trajetórias parametrizada pelo inverso da temperatura $\beta$, e $\beta_{c}$ a temperatura inversa crítica, o propósito do projeto é investigar a convergência $\left(y=\left(y^{(1)}, \ldots, y^{(N)}\right) \in \mathbb{R}^{N}\right)$

$$
\lim _{k \rightarrow \infty} \sigma_{k}^{(N)}\left(y ; \beta_{c}\right)=\sigma^{\text {gauss }}(y)=\frac{1}{(2(L+1) \pi)^{N / 2}} \int_{-\infty}^{y^{(1)}} \cdots \int_{-\infty}^{y^{(N)}} e^{-\frac{1}{2(L+1)}\left|y^{\prime}\right|^{2}} d^{N} y^{\prime}
$$

da trajetória crítica $\left\{\sigma_{k}^{(N)}\left(y ; \beta_{c}\right)\right\}_{k=1}^{\infty}$ para a distribuição gaussiana $\sigma^{\text {gauss }}(y)$ quando se parte da condição inicial definida pelo modelo (integrando-se (1.0.3))

$$
\sigma_{0}^{(N)}(y)=\left\{\begin{array}{lll}
0 & \text { se } & |y|<\sqrt{\beta N} \\
1 & \text { se } & |y| \geq \sqrt{\beta N}
\end{array}\right.
$$

e $N$ é muito grande; em outras palavras, estabelecer o Teorema Central do Limite para o modelo $O(N)$ de Heisenberg hierárquico na criticalidade para $N$ suficientemente grande. Note que $\sigma^{\text {gauss }}(y)$ é um ponto fixo do mapa (1.0.5), chamado de ponto fixo gaussiano ou ponto fixo trivial.

Apesar de modelos hierárquicos terem sido extensivamente estudados nas décadas de 70 e 80, os trabalhos se restringem a uma análise local de (1.0.5), isto é, a dinâmica é estudada partindo-se de uma vizinhança do ponto fixo. Somente recentemente a análise 
global dessa equação tem sido empregada - vide [25] e [49], e mais recentemente nossa contribuição [41]. Essas referências estão alinhadas com a seguinte direção:

Direção 1.0.1 (Análise Global das Trajetórias) Estudo da trajetória do grupo de renormalização partindo da condição inicial definida pelo modelo. Em especial, estudo da trajetória crítica (quando $\beta=\beta_{c}$ ) - os modelos a serem tratados exibem transição de fase, existindo assim uma temperatura inversa crítica $\beta_{c}$ (que depende da dimensão espacial em que o modelo é definido) que separa comportamentos distintos do sistema. Devemos enfatizar que a determinação da superfície crítica por um único parâmetro (a temperatura) é bastante complicada, mesmo quando se investiga as trajetórias na vizinhança de um ponto fixo, vizinhança essa que, em geral, não contém a condição inicial original do modelo. O objetivo aqui é estabelecer critérios para determinar a trajetória crítica mesmo partindo de uma condição inicial distante do ponto fixo.

Teorema de Lee-Yang. Seja $d \nu_{n}(\boldsymbol{y})$ a medida de Gibbs (medida de equilíbrio) de um sistema ferromagnético clássico de $|\Lambda|=n$ spins, com $\boldsymbol{y}=\left(y_{1}, \ldots, y_{n}\right)$ um elemento do espaço de configuração $\Omega_{n}=\mathbb{R} \times \cdots \times \mathbb{R}=\mathbb{R}^{n}$, e consideremos a função característica (transformada de Fourier) dessa medida

$$
\Phi_{n}(\boldsymbol{z} ; \beta)=\int_{\Omega_{n}} \exp \left\{i(\boldsymbol{z}, \boldsymbol{y})_{\Omega_{n}}\right\} d \nu_{n}(\boldsymbol{y}),
$$

com $\boldsymbol{z}=\left(z_{1}, \ldots, z_{n}\right)$ a variável conjugada. Segundo Lee e Yang, as propriedades de equilíbrio termodinâmico do sistema são regidas pela distribuição dos zeros (zeros de Lee-Yang) de

$$
\varphi_{n}(x ; \beta):=\Phi_{n}(z, \ldots, z ; \beta), \quad x=-|z|^{2} .
$$

No artigo [40] de 1952 Lee e Yang calculam, por uma analogia com a eletrostática, a densidade de zeros do modelo de Ising unidimensional e derivam, a partir dessa, a energia livre e a magnetização. Embora o modelo não apresente transição de fase, o cálculo ilustra o adensamento dos zeros, pelo limite termodinâmico $n \rightarrow \infty$, sobre uma curva no plano complexo e explicita a maneira como as propriedades macroscópicas de 
equilíbrio do modelo são determinadas a partir da densidade (distribuição empírica) dos zeros de (1.0.6).

Em 1974 Newman [44] formulou esse problema da determinação dos zeros de LeeYang da seguinte maneira: se $\varphi_{n}(x ; 0)$ possui zeros sobre a reta real, então $\varphi_{n}(x ; \beta)$ mantém os zeros sobre $\mathbb{R}$ para $\beta>0$ e todo $n$, e esses zeros possivelmente se adensam no limite $n \rightarrow \infty$ - essa questão do adensamento somente foi levada em consideração por De Coninck [11], e será explicada mais adiante (vide páginas 26 e 27).

Teorema de Lee-Yang e o Modelo $O(N)$ de Heisenberg Hierárquico. As funções termodinâmicas do modelo $O(N)$ de Heisenberg hierárquico dependem das variáveis macroscópicas $X_{k}^{(N)}(1.0 .3)$. Para esse modelo temos a seguinte relação

$$
\begin{aligned}
\Phi_{m}^{(N)}\left(\frac{z}{\sqrt{m^{1+2 / d}}}, \ldots, \frac{z}{\sqrt{m^{1+2 / d}}}\right) & =\int_{\Omega_{m}} \exp \left\{i z \cdot X_{k}^{(N)}\right\} d \nu_{m}^{(N)}(\boldsymbol{y}) \\
& =\int_{\mathbb{R}^{N}} \exp \{i z \cdot y\} d \sigma_{k}^{(N)}(y)=\phi_{k}^{(N)}(z),
\end{aligned}
$$

com $d \nu_{m}^{(N)}(\boldsymbol{y})$ a medida de Gibbs para um bloco de $m=L^{k d}$ spins e $\sigma_{k}^{(N)}(y)$ a distribuição "a priori" da variável soma $X_{k}^{(N)}$. Para um estudo do modelo à luz do Teorema de Lee-Yang é portanto suficiente que se conheça os zeros da função característica $\phi_{k}^{(N)}(z)$, $k=0,1,2, \ldots$, quando escrita como função de $-|z|^{2}$. Define-se nesse contexto a chamada propriedade de Lee-Yang (vide Definição 2.2.8): diz-se que uma medida de probabilidade $\sigma$ em $\mathbb{R}^{N}$ invariante por transformações ortogonais $O(N)$ possui a propriedade de LeeYang se sua função característica $\phi(z)=\int_{\mathbb{R}^{N}} \exp \{i z \cdot y\} d \sigma(y)=\varphi(x), \operatorname{com} x=-|z|^{2}$, é tal que possui a representação

$$
\varphi(x)=\exp (\lambda x) \prod_{n=1}^{\infty}\left(1+\frac{x}{\gamma_{n}}\right)
$$

com $\lambda \geq 0$ e $\left\{-\gamma_{n}, n \geq 1\right\}$ números reais não-positivos tais que $-\gamma_{n+1} \leq-\gamma_{n} \leq 0$ para todo $n=1,2,3, \ldots e \sum_{n=1}^{\infty} \gamma_{n}^{-1}<\infty$; em outras palavras, $\varphi(x)$ pertence à classe

$$
\mathcal{L}^{(1)}=\{f \in \mathcal{L}: f(0)=1\},
$$


sendo $\mathcal{L}$ a classe das funções inteiras de Laguerre (vide Definição 2.2.7) - tal classe é formada por polinômios que possuem somente zeros reais não-positivos, bem como os limites uniformes desses polinômios sobre subconjuntos compactos de $\mathbb{C}$.

Convergência da Seqüência $\left\{\sigma_{k}^{(N)}\left(y ; \beta_{c}\right)\right\}_{k=1}^{\infty}$. A abordagem apresentada neste projeto, bem como nos trabalhos de Kozitsky [32] e Watanabe [49], envolve técnicas de grupo de renormalização, segundo as quais a convergência mencionada é investigada seguindo-se a trajetória crítica $\left\{\phi_{k}^{(N)}\left(z ; \beta_{c}\right)\right\}_{k=1}^{\infty}$ das funções características das medidas $\left\{\sigma_{k}^{(N)}\left(y ; \beta_{c}\right)\right\}_{k=1}^{\infty}$, partindo-se de alguma condição inicial. Em termos de $\phi_{k}^{(N)}(z),(1.0 .5)$ fica (vide Proposição 2.2.4)

$$
\phi_{k}^{(N)}(z)=\frac{1}{N_{k}} \exp \left\{-\frac{(L-1)}{2} \Delta\right\}\left(\phi_{k-1}^{(N)}\left(L^{-(1+d / 2)} z\right)\right)^{L^{d}}
$$

sendo $\Delta=\partial^{2} / \partial z_{1}^{2}+\cdots+\partial^{2} / \partial z_{N}^{2}$ o operador laplaceano $N$-dimensional e $N_{k}$ uma normalização tal que $\phi_{k}^{(N)}(0)=1$ para todo $k$. Note que

$$
\phi^{\text {gauss }}(z)=\int_{\mathbb{R}^{N}} \exp \{i z \cdot y\} d \sigma^{\text {gauss }}(y)=e^{-\frac{L+1}{2}|z|^{2}}
$$

é o ponto fixo gaussiano (ou trivial) de (1.0.8).

Um ingrediente chave no estudo da seqüência $\left\{\phi_{k}^{(N)}\left(z ; \beta_{c}\right)\right\}_{k=1}^{\infty}$ gerada por (1.0.8) decorre do seguinte resultado (vide referência [29]): seja $L^{d}$ um inteiro e $\chi$ uma medida de probabilidade que possui a propriedade de Lee-Yang; se $\chi$ é tomada como condição inicial para o mapa (1.0.5), então $\sigma_{k}^{(N)}(y)$, para todo $k=1,2,3, \ldots$, também possui a propriedade de Lee-Yang. Pela definição de propriedade de Lee-Yang, esse teorema nos diz que se

$$
h(z)=\int_{\mathbb{R}^{N}} \exp \{i z \cdot y\} d \chi(y)=g(x)
$$

com $g(x)$ pertencente à classe $\mathcal{L}^{(1)}$, é tomada como condição inicial para (1.0.8), então para todo $k=1,2,3, \ldots$ tem-se que

$$
\phi_{k}^{(N)}(z)=\int_{\mathbb{R}^{N}} \exp \{i z \cdot y\} d \sigma_{k}^{(N)}(y)=\varphi_{k}^{(N)}(x)
$$


com $\varphi_{k}^{(N)}(x)$ pertencente à classe $\mathcal{L}^{(1)}$. Agora, para essa classe de funções existem algumas desigualdades devidas à Newman (vide Teorema 6 de [44]): partindo da representação (1.0.7) temos que

$$
\varphi_{k}^{(N)}(x)=\exp \left(\sum_{j=1}^{\infty} \nu_{j, k}^{(N)} x^{j}\right)
$$

com

$$
\nu_{1, k}^{(N)}=\lambda_{k}^{(N)}+\sum_{n=1}^{\infty}\left(\gamma_{n, k}^{(N)}\right)^{-1} \quad \text { e } \quad \nu_{j, k}^{(N)}=\frac{(-1)^{j+1}}{j} \sum_{n=1}^{\infty}\left(\gamma_{n, k}^{(N)}\right)^{-j} \quad(j \geq 2)
$$

sendo que essa expansão é válida para todo $|x|<\gamma_{1, k}^{(N)}$; o teorema de Newman diz que

$$
0 \leq(-1)^{j+1} j \nu_{j, k}^{(N)} \leq\left(-2 \nu_{2, k}^{(N)}\right)^{j / 2}, \quad j \geq 3
$$

Se ao longo de uma trajetória $\left\{\varphi_{k}^{(N)}(x)\right\}_{k=1}^{\infty}$ o coeficiente $\nu_{2, k}^{(N)}$ convergir para zero, então todos coeficientes de ordem superior $\nu_{j, k}^{(N)}, j \geq 3$, convergem igualmente para zero. Nesse caso teríamos a convergência $\varphi_{k}^{(N)}(x) \longrightarrow e^{\nu_{1, \infty}^{(N)} x}$ (isto é, $\phi_{k}^{(N)}(z) \longrightarrow e^{-\nu_{1, \infty}^{(N)}|z|^{2}}$ ).

Apresentados esses fatos, vejamos as contribuições de Kozitsky e Watanabe:

Em [32], Kozitsky estabelece o Teorema Central do Limite para o modelo $O(N)$ de Heisenberg hierárquico na criticalidade $\left(\phi_{k}^{(N)}\left(z ; \beta_{c}\right) \rightarrow \phi^{\text {gauss }}(z)\right)$ para todo $N \geq 0$, $4<d<\infty, L^{d} \geq 2$ um inteiro, partindo de uma condição inicial $h(z)=g(x) \operatorname{com} g(x)$ pertencente a uma subclasse de $\mathcal{L}^{(1)}$ que depende de $d$ (vide Definição 2.2.17). Como já explicado, o fato de se ter $L^{d}$ um inteiro garante que a propriedade de Lee-Yang é preservada e, conseqüentemente, tem-se à disposição a desigualdade (1.0.9) para o controle da convergência dos cumulantes (vide Comentário 2.2.13). Um ponto importante esclarecido nesse artigo é o seguinte: a convergência da seqüência de funções características $\left\{\phi_{k}^{(N)}(z ; \beta)\right\}_{k=1}^{\infty}$ para o ponto fixo gaussiano ocorre somente se $\beta=\beta_{c}$; se $\beta>\beta_{c}$, a seqüência diverge; se $\beta<\beta_{c}$, a seqüência converge para a função identicamente igual a 1 (note que 1 é um ponto fixo de (1.0.8)).

Já em [49], Watanabe estabelece o Teorema Central do Limite para o modelo $O(N)$ de Heisenberg hierárquico na criticalidade para $N$ suficientemente grande, $4 \leq d<\infty$ 
(note a inclusão de $d=4$ ), $L^{d}=2$, partindo da função característica da medida "a priori" $d \sigma_{0}^{(N)}(y)$ (vide $\left.(1.0 .3)\right)$

$$
\begin{aligned}
\phi_{0}^{(N)}(z) & =\int_{\mathbb{R}^{N}} \exp \{i z \cdot y\} d \sigma_{0}^{(N)}(y) \\
& =\frac{\Gamma(N / 2)}{(\sqrt{\beta N}|z| / 2)^{N / 2-1}} J_{N / 2-1}(\sqrt{\beta N}|z|),
\end{aligned}
$$

que é a condição inicial definida pelo próprio modelo - se $\left\{ \pm \alpha_{n, N / 2-1}, n \geq 1\right\}$ denotam os zeros positivos e negativos de $J_{N / 2-1}(\xi)$ (função de Bessel de ordem $N / 2-1$ ), mostramos na Proposiãa 2.2 .9 que $\varphi_{0}^{(N)}(x)\left(\phi_{0}^{(N)}(z)\right.$ escrita em termos de $\left.x=-|z|^{2}\right)$ possui a representação

$$
\varphi_{0}^{(N)}(x)=\prod_{n=1}^{\infty}\left(1+\frac{x}{\frac{\alpha_{n, N / 2-1}^{2}}{\beta N}}\right)
$$

e portanto $\varphi_{0}^{(N)} \in \mathcal{L}^{(1)}$. Para controlar a trajetória $O(N)$, que parte de muito longe do ponto fixo gaussiano, Watanabe utiliza a trajetória exatamente solúvel $O(\infty)$ juntamente com dois ingredientes válidos para $L^{d}$ inteiro: o fato de o mapa preservar a propriedade de Lee-Yang (e por conseguinte podemos fazer uso da desigualdade (1.0.9)), e a propriedade de positividade por reflexão (o que permite o emprego de um resultado devido a Kupiainen [35] sobre a expansão $1 / N$, e que garante a convergência uniforme da trajetória $O(N)$ para a trajetória $O(\infty)$ - vide Comentário 2.2.20).

A Aproximação de Potencial Local $(L \downarrow 1)$. Embora a transformação (1.0.8) seja discreta, em ambos os artigos ([32] e [49]) parte da dinâmica é evoluída através da equação do calor. O presente trabalho diferencia-se desses dois pela utilização da chamada aproximação de potencial local: definindo a função

$$
U(t, z)=-\ln \phi_{k}^{(N)}(z)
$$

com parâmetro de escala 


$$
t=k \ln L
$$

toma-se conjuntamente os limites $k \rightarrow \infty$ e $L \downarrow 1$ de tal maneira que $k \ln L$ permaneça fixo em um número real positivo $t$. Com tal procedimento, a órbita discreta $\left\{-\ln \phi_{k}^{(N)}(z)\right\}_{k=0}^{\infty}$ torna-se contínua, e a dinâmica passa a ser gerada pela seguinte equação (vide Proposição 2.3.1):

$$
U_{t}=-\frac{1}{2}\left(\Delta U-\left|U_{z}\right|^{2}\right)-\frac{d+2}{2} z \cdot U_{z}+d U+\left.\frac{1}{2}\left(\Delta U-\left|U_{z}\right|^{2}\right)\right|_{z=0}
$$

$t \in \mathbb{R}_{+}$e $z \in \mathbb{R}^{N}$, com condição inicial

$$
U(0, z)=-\ln \left[\frac{\Gamma(N / 2)}{(\sqrt{\beta N}|z| / 2)^{N / 2-1}} J_{N / 2-1}(\sqrt{\beta N}|z|)\right]
$$

- $U(0, z)=-\ln \phi_{0}^{(N)}(z), \operatorname{com} \phi_{0}^{(N)}(z)$ dada por (1.0.10). O último termo no lado direito de (1.0.12) garante que $U(t, 0)=0$ para todo $t \geq 0$ - note que essa propriedade é satisfeita pela condição inicial, já que $\phi_{0}^{(N)}(0)=1$. Essa equação de evolução descreve a transformação de grupo de renormalização do modelo $O(N)$ de Heisenberg hierárquico em um espaço de dimensão $d$ a uma temperatura inversa $\beta$ : ela determina a função característica $\phi^{(N)}(t, z)=\exp (-U(t, z))$ da distribuição da variável de spin de bloco na escala $t$. Note que

$$
U^{\text {gauss }}(z)=\lim _{L \downarrow 1}\left\{-\ln \phi^{\text {gauss }}(z)\right\}=|z|^{2}
$$

é o ponto fixo gaussiano (ou trivial) de (1.0.12).

A função inicial (1.0.13) depende de $|z|$ e a simetria esférica é preservada por (1.0.12). Logo, é conveniente e suficiente que trabalhemos com a variável radial $x=-|z|^{2}$. Desse fato, e levando em conta que queremos tomar $N$ para infinito, definimos a função adequadamente escalada em $N$

$$
u^{(N)}(t, x)=\frac{1}{N} U(t, \sqrt{N} z)
$$


Em termos desse, (1.0.12)-(1.0.13) ficam (vide Proposição 2.3.3)

$$
u_{t}^{(N)}=\frac{2}{N} x u_{x x}^{(N)}+u_{x}^{(N)}-2 x\left(u_{x}^{(N)}\right)^{2}-(d+2) x u_{x}^{(N)}+d u^{(N)}-u_{x}^{(N)}(t, 0),
$$

aqui denominada de $\mathrm{E} u^{(N)}$, com

$$
u^{(N)}(0, x)=-\frac{1}{N} \ln \left[\frac{\Gamma(N / 2)}{(i \sqrt{\beta x} N / 2)^{N / 2-1}} J_{N / 2-1}(i \sqrt{\beta x} N)\right] .
$$

O último termo em (1.0.14) garante que $u^{(N)}(t, 0)=0$ para todo $t \geq 0$. Note que

$$
u^{\text {gauss }}(x)=\frac{1}{N} U^{\text {gauss }}(\sqrt{N} z)=-x
$$

é o ponto fixo gaussiano (ou trivial) de (1.0.14).

O problema de valor inicial (1.0.14)-(1.0.15) é o ponto de partida de nossos estudos. Nosso objetivo pode ser enunciado da seguinte maneira: fixado $\beta$ na temperatura inversa crítica $\beta_{c}=\beta_{c}(d, N)$, mostrar que a trajetória crítica $\left\{u^{(N)}(t, x), t \geq 0, \beta=\beta_{c}\right\}$ permanece sobre uma variedade estável e converge para o ponto fixo gaussiano $u^{\text {gauss }}(x)$ quando $t \rightarrow \infty$ (limite termodinâmico) se $N$ é suficientemente grande e $4 \leq d<\infty$. Devemos enfatizar que em decorrência do fato que no limite $L \downarrow 1$ a propriedade de Lee-Yang e a propriedade de positividade por reflexão deixam de valer, um método inteiramente novo tem sido desenvolvido em nossas análises.

O Caso $N=\infty$. Assim como no programa de Watanabe [49], também devemos lidar com a trajetória crítica no limite $N \rightarrow \infty$ - quando $N=\infty$ o modelo recebe o nome de modelo esférico hierárquico (vide [8]). O estudo desse caso foi realizado entre os anos de 2006 e 2008, e deu origem ao artigo [41] e à dissertação de mestrado [7]. Embora tenhamo-nos concentrado no caso $d=4$, todos os resultados são válidos para $4 \leq d<\infty$. O principal teorema diz que a trajetória crítica (exatamente solúvel)

$$
\left\{u^{(\infty)}(t, x), t \geq 0, \beta=\beta_{c}(4)=4\right\}
$$




$$
u^{(\infty)}(0, x)=\lim _{N \rightarrow \infty} u^{(N)}(0, x)=\int_{0}^{x} \frac{-\beta}{1+\sqrt{1+4 \beta x^{\prime}}} d x^{\prime}
$$

permanece sobre uma variedade estável e converge para o ponto fixo gaussiano $u^{\text {gauss }}(x)$ quando $t \rightarrow \infty$. Embora tenhamos usado a expressão "exatamente solúvel", (1.0.16) não foi estudada diretamente, mas pela trajetória crítica $\left\{v^{(\infty)}(t, p), t \geq 0, \beta=\beta_{c}(4)=4\right\}$, sendo $v^{(\infty)}(t, p)=w_{p}^{(\infty)}(t, p) \mathrm{e}$

$$
w^{(\infty)}(t, p)=\max _{x}\left(x p-u^{(\infty)}(t, x)\right)=\bar{x} p-u^{(\infty)}(t, \bar{x})
$$

a transformada de Legendre de $u^{(\infty)}(t, x)$ com respeito à variável $x$. Trabalhar com $v^{(\infty)}(t, p)$ mostrou-se bastante vantajoso. Um dos pontos que merece destaque é a determinação da temperatura inversa crítica a partir da EDP satisfeita por $v^{(\infty)}(t, p)^{1}$ : para $d>2$ qualquer

$$
\beta_{c}(d)=\frac{2 d}{d-2}
$$

Como já foi dito na Direção 1.0.1, detectar a superfície crítica em uma transformação de grupo de renormalização é uma tarefa bastante difícil, mesmo na vizinhança de $u^{\text {gauss }}(x)$. Vale lembrarmos que o método utilizado para modelos hierárquicos é devido a BleherSinai. Outro ponto que merece destaque é o fato de o estudo de $v^{(\infty)}(t, p)$ do ponto de vista da teoria geométrica de funções permitir a descrição precisa da dinâmica das singularidades de $u_{x}^{(\infty)}(t, x)$. Essa investigação diz respeito à seguinte direção:

Direção 1.0.2 (Soluções no Plano Complexo) Extensão da solução da equação a derivadas parciais para o plano complexo com o propósito de estudar a evolução de suas singularidades, cortes e zeros. Nesse panorama, temos interesse em saber se a classe $P$ das funções de Pick é preservada - trata-se de funções $f(x)$ analíticas no semi-plano superior $\mathbb{H}$ que apresentam parte imaginária positiva se o argumento está em $\mathbb{H}$, isto é, $\Im m(f) \geq 0$ se $\Im m(x)>0$. A referida classe de funções é suficientemente abrangente,

\footnotetext{
${ }^{1}$ Até então a temperatura inversa crítica tinha sido encontrada pelo método assintótico de Kac vide referência [8].
} 
incluindo funções meromorfas (isto é, possuem zeros e pólos) e funções que possuem cortes sobre o eixo real.

A classe $P$ aparece no estudo da condição inicial $u_{x}^{(N)}(0, x)=\sqrt{\frac{\beta}{4 x}} \frac{i J_{N / 2}(i \sqrt{\beta x} N)}{J_{N / 2-1}(i \sqrt{\beta x} N)}$ : pela definição de $u^{(N)}(t, x)$ e por (1.0.11) segue que

$$
\begin{aligned}
u_{x}^{(N)}(0, x) & =\frac{d}{d x}\left(-\frac{1}{N} \ln \varphi_{0}^{(N)}(N x)\right) \\
& =-\frac{1}{N} \sum_{n=1}^{\infty} \frac{1}{\frac{\alpha_{n, N / 2-1}^{2}}{\beta N^{2}}+x}
\end{aligned}
$$

que é a representação de uma função meromorfa com pólos de massa $1 / N$ localizados em $\left\{-\alpha_{n, N / 2-1}^{2} /\left(\beta N^{2}\right), n \geq 1\right\}$ - os zeros de $\varphi_{0}^{(N)}(N x)$ passam a ser os pólos de $u_{x}^{(N)}(0, x)$. Mostramos que a seqüência de condições iniciais $\left(u_{x}^{(N)}(0, x)\right)_{N \geq 3}$, bem como seu limite

$$
u_{x}^{(\infty)}(0, x)=\lim _{N \rightarrow \infty} u_{x}^{(N)}(0, x)=\frac{-\beta}{1+\sqrt{1+4 \beta x}}
$$

pertencem à classe $P$ - a interpretação que fizemos é que no limite $N \rightarrow \infty$ os pólos de $u_{x}^{(N)}(0, x)$ se adensam, formando o corte apresentado por $u_{x}^{(\infty)}(0, x)$. Para essa classe existe uma representação integral canônica; para $u_{x}^{(\infty)}(0, x) \operatorname{com} \beta=\beta_{c}(4)=4$ temos

$$
u_{x}^{(\infty)}(0, x)=\int_{-\infty}^{+\infty} \frac{1}{\lambda-x} d \mu^{(\infty)}(\lambda)
$$

com

$$
d \mu^{(\infty)}(\lambda)=\frac{4}{\pi} \frac{\sqrt{16(-\lambda)-1}}{16(-\lambda)} d \lambda
$$

uma medida de Borel positiva absolutamente contínua com respeito à medida de Lebesgue $d \lambda$, suportada sobre o intervalo $(-\infty,-1 / 16)$ (o corte de $u_{x}^{(\infty)}(0, x)$ ).

Com essas constatações, a pergunta seguinte foi: a classe $P$ das funções de Pick é preservada pela dinâmica de $u_{x}^{(\infty)}(t, x)$ ? A resposta encontra foi positiva, e demos a representação integral (não canônica) 


$$
u_{x}^{(\infty)}(t, x)=-1+\int_{-\infty}^{+\infty}\left(\frac{1}{\lambda-x}-\frac{1}{\lambda-1 / 2}\right) d \mu^{(\infty)}(t, \lambda)
$$

sendo que o modo de se determinar a medida $d \mu^{(\infty)}(t, \lambda)=\rho(t, \lambda) d \lambda$ é o mesmo da representação integral canônica. Essa medida é uma medida de Borel positiva absolutamente contínua com respeito à medida de Lebesgue $d \lambda$, suportada sobre $\Sigma(t)=\left(-\infty, x^{\dagger}(t)\right)$, o corte de $u_{x}^{(\infty)}(t, x)$. Mostramos que $x^{\dagger}(t)$ é uma função monótona decrescente de $t$, $\operatorname{com} x^{\dagger}(0)=-1 / 16$ e $\lim _{t \rightarrow \infty} x^{\dagger}(t)=-\infty$; desse limite temos a informação que o corte é expelido para o infinito negativo no limite $t \rightarrow \infty$. Assim, o suporte $\Sigma(t)$ vai para o conjunto vazio quando $t \rightarrow \infty$, de modo que a integral da representação converge para 0 uniformemente em todo compacto $K \in \mathbb{H}$. Em outras palavras, $u_{x}(t, x)$ converge para a função inteira $u_{x}^{\text {gauss }}(x) \equiv-1$ no limite (termodinâmico) $t \rightarrow \infty$.

Por fim, lembrando que $\phi^{(N)}(t, z)=\exp (-U(t, z))$ e fazendo $\varphi^{(N)}(t, x)=\phi^{(N)}(t, z)$, deparamo-nos com a seguinte questão: a função

$$
\exp \left(-u^{(\infty)}(t, x)\right)=\lim _{N \rightarrow \infty}\left(\varphi^{(N)}(t, N x)\right)^{1 / N}
$$

é a transformada de Fourier de uma medida positiva em $\mathbb{R}$ ? Nesse contexto é importante o conceito de distribuições de probabilidade infinitamente divisíveis. Tal questão contribui na seguinte direção:

Direção 1.0.3 (Divisibilidade Infinita) Uma distribuição de probabilidade sobre $\mathbb{R}_{+}$ é dita ser infinitamente divisível se, e somente se, para todo número natural $N$ sua transformada de Laplace (ou Fourier) elevada a $1 / N$ é a transformada de Laplace (ou Fourier) de uma distribuição de probabilidade. A função característica (transformada de Fourier da medida de probabilidade) de uma distribuição infinitamente divisivel é chamada de função característica infinitamente divisível. Um fato importante é que, se uma sequência de funções características infinitamente divisíveis converge para alguma função característica, então essa última é também infinitamente divisível ([45]). Esse é um dos pontos explorados em [11], no contexto de sistemas ferromagnéticos ddimensionais com interação entre pares, para uma classe ampla de distribuições de spin (livres) - são consideradas distribuições de spin que são simétricas, possuem suporte limitado e satisfazem a propriedade de Lee-Yang. O trabalho de De Coninck estabelece 
uma relação entre energia livre e o logaritmo de uma função característica infinitamente divisivel, válida inclusive no limite termodinâmico. Essa relação (como demonstramos para o modelo esférico hierárquico - vide [7] ou [41]) é preservada pela dinâmica de grupo de renormalização e pode ser expressa em termos da teoria da representação das funções de Pick.

Para respondermos a nossa questão lançamos mão do seguinte teorema, encontrado na Seção 7 do Capítulo XIII do livro "An Introduction to Probability Theory and Its Applications", Vol. 2, 2 ${ }^{\mathrm{a}}$ edição, Wiley, New York (1971), de William Feller (vide página 450, Teorema 1):

Teorema 1.0.4 Uma função $f(x)$ é a transformada de Laplace de uma distribuição de probabilidade infinitamente divisivel sobre $\mathbb{R}_{+}$se, e somente se, $f(x)=e^{-h(x)}$ com $h\left(0^{+}\right)=0$ e $(-1)^{m} \frac{d^{m} h^{\prime}(x)}{d x^{m}} \geq 0$ para $x \in \mathbb{R}_{+}$e todo $m=0,1,2, \ldots$

A nossa conclusão é que $F(t, x)=\exp \left(+u^{(\infty)}(t, x)\right)$ é a transformada de Laplace de uma distribuição de probabilidade infinitamente divisível sobre $\mathbb{R}_{+}$, haja vista que:

(i) $u^{(\infty)}(t, 0)=0$ para todo $t \geq 0$ (vide comentário abaixo de (1.0.14)-(1.0.15));

(ii) é possível verificar que $-u_{x}^{(\infty)}(t, x) \geq 0$ para todo $t \geq 0$ e $x \in \mathbb{R}_{+}$;

(iii) pela representação (1.0.17) (lembrando que a medida é positiva) segue que

$$
(-1)^{m} \frac{\partial^{m}-u_{x}^{(\infty)}(t, x)}{\partial x^{m}}=\int_{-\infty}^{+\infty} \frac{m !}{(x-\lambda)^{m+1}} d \mu^{(\infty)}(t, \lambda) \geq 0
$$

para todo $t \geq 0, x \in\left(x^{\dagger}(t), \infty\right)$ (e em particular em $\mathbb{R}_{+}$, pois $x^{\dagger}(t) \leq-1 / 16$ ) e $m=1,2,3, \ldots$.

O Caso $N>>1$ Finito. Sobre (1.0.14), trata-se de uma equação não-linear que apresenta o termo $1 / N$ na frente do termo de derivada segunda. A equação com $N$ finito é, por conseguinte, uma perturbação singular da equação com $N=\infty$ : com o limite $N \rightarrow \infty$ a ordem da equação é reduzida de segunda para primeira. Uma questão natural que se apresenta à nossa investigação é: qual o sentido da série formal da solução da $\mathrm{E} u^{(N)}$ em potências de 2/N? Acabamos por ter contato com as referências [3] e 
[39] entre tantas outras, as quais tratam da chamada somabilidade de séries formais; em especial, a primeira citada trata do estudo da somabilidade de séries formais que satisfazem uma dada EDO, EDO essa que apresenta uma perturbação singular. Tais questões estão alinhadas com a seguinte direção:

Direção 1.0.5 (Somabilidade da Série Formal em 2/N) Através de um método de somabilidade, transformar a série formal em $\varepsilon=2 / N$ da solução da equação de grupo de renormalização, aqui estendida para um setor do plano complexo, na única solução analítica. Como discutido na introdução do artigo [4], um método de somabilidade pode ser visto como um mapa linear $\mathcal{S}$ definido sobre algum espaço vetorial $X$ de séries; tomaremos séries formais de potências na variável complexa $\varepsilon$. A cada série formal dessas, $\mathcal{S}$ associa uma soma generalizada, que para séries de potências em $\varepsilon$ devem ser funções holomorfas nessa variável. Para ser útil em aplicações a equações diferenciais, o operador $\mathcal{S}$ deve ter algumas propriedades:

- O espaço $X$ deve conter todas as séries de potências convergentes, e $\mathcal{S}$ deve mapear cada série convergente em sua soma natural.

- O espaço linear $X$ deve ser uma álgebra diferencial; isto é, para cada série de potências em $X$, sua derivada formal deve também pertencer a $X$, e para quaisquer duas séries de potências em $X$, o produto delas deve também estar em $X$.

- O operador $\mathcal{S}$ deve ser um homomorfismo de álgebras diferenciais; assim, deve ser não apenas linear, mas deve mapear produtos em produtos, e derivadas em derivadas.

- Para qualquer série formal de potências $\hat{f} \in X$, a função $\mathcal{S} \hat{f}$ deve ser holomorfa em algum domínio setorial $S$, e assintótica a $\hat{f}$ quando $\varepsilon \rightarrow 0$ em $S$.

Uma variante da chamada somabilidade de Borel preenche todos esses requisitos - vide [1] $e[4]$.

Como foi dito acima, a função obtida pelo método de somabilidade é holomorfa em algum domínio setorial $S$, sendo que a unicidade dessa função é garantida se o setor $S$ tiver uma abertura ampla; além disso, a função holomorfa obtida é assintótica à série formal quando $2 / N=\varepsilon \rightarrow 0 \mathrm{em} S$, o que significa que o estudo da série formal através apenas de seus primeiros termos é justificada. Todos esses conceitos serão apresentados claramente no Capítulo 3. 


\section{Conteúdo desta Tese}

O presente trabalho é destinado ao estudo da condição inicial $u_{x}^{(N)}(0, x)$ e da função $v^{(N)}(t, p)$ para $t>0$, sendo $v^{(N)}(t, p)$ a função inversa de $u_{x}^{(N)}(t, x)$ com respeito à variável $x$, no que diz respeito à somabilidade de suas séries formais em potências de $2 / N$ - a razão de estudarmos $v^{(N)}(t, p)$, e não $u_{x}^{(N)}(t, x)$, para $t>0$ será elucidada posteriormente. Ao longo deste trabalho adotaremos a notação $\varepsilon=2 / N$, sendo que $\varepsilon$ sempre pertencerá a um setor do plano complexo. Quando utilizarmos a variável $\varepsilon$, passaremos a escrever $u_{x}^{(N)}(t, x)$ e $v^{(N)}(t, p)$ respectivamente como $u_{x}(\varepsilon, t, x)$ e $v(\varepsilon, t, p)$.

O Capítulo 2 é formado por três seções. Na Seção 2.1 definimos o modelo $O(N)$ de Heisenberg hierárquico: definimos de modo cuidadoso a interação hierárquica e a medida de Gibbs do modelo. Fazendo uso da estrutura da energia hierárquica, achamos na Seção 2.2 uma relação recursiva para a chamada medida "a priori" e, a partir dessa, uma relação recursiva para a transformada de Fourier da medida "a priori". É pela função característica da medida que se faz ponte com a teoria de Lee-Yang. Com esse próposito definimos propriedade de Lee-Yang. Para fechar essa seção expomos parte de um artigo de Kozitsky, além de fazermos um comentário sobre um trabalho de Watanabe. A Seção 2.3, que encerra o primeiro capítulo, trata da chamada aproximação de potencial local, e somos levados a uma equação a derivadas parciais para um potencial associado às funções características acima mencionadas.

No Capítulo 3 apresentamos algumas definições, um teorema básico e importante, e algumas ferramentas que serão de extrema importância neste trabalho. Basicamente, seguimos o Parágrafo 1 de [39]. Também apresentamos nosso primeiro teorema (Teorema 3.4.1): ele trata das propriedades que podem ser inferidas para uma função $g(\varepsilon, w)$ a partir das propriedades verificadas para sua inversa com respeito à variável $w, f(\varepsilon, z)=$ $g^{-1}(\varepsilon, z)$. Mais especificamente, as propriedades de ser analítica em $S \times D$ e Gevrey ordem $s$ assintoticamente expansiva em $S, S$ um setor e $D$ um disco do plano complexo. De todas as referências com as quais tivemos contato, nenhuma trata dessa questão. Entretanto, em nossos estudos, esse problema de se obter informações de $g(\varepsilon, w)$ a partir de $f(\varepsilon, z)=g^{-1}(\varepsilon, z)$ aparece naturalmente: queremos estudar a função $u_{x}(\varepsilon, t, x)$; para a condição inicial é mais simples e transparente (algumas questões ficam mais claras) trabalhar diretamente com $u_{x}(\varepsilon, 0, x)$; já para o caso $t>0$, só temos condições 
de trabalhar com $v(\varepsilon, t, p)$, que como já foi dito no parágrafo acima, é a inversa de $u_{x}(\varepsilon, t, x)$ com respeito à variável $x$. Assim sendo, nossa estratégia é que, encerradas as investigações sobre a função $v(\varepsilon, t, p)$, evoquemos o Teorema 3.4 .1 e tiremos conclusões sobre a função $u_{x}(\varepsilon, t, x)$. Como julgamos que esse teorema é interessante por si, estamos organizando-o em um artigo ([9]).

O Capítulo 4 é devotado à condição inicial $u_{x}(\varepsilon, 0, x)$, e o Capítulo 5 à $v(\varepsilon, t, p)$, que corresponde ao caso $t>0$. Maiores detalhes serão dados no início desses capítulos.

Um ponto que merece destaque, apresentado no Capítulo 5, é a questão de se encontrar a superfície crítica fixando o parâmetro $\beta$ : essa é abordada ordem a ordem em $\varepsilon$. Considerando a série (formal)

$$
\beta(\varepsilon)=\sum_{i=0}^{\infty} \beta_{i} \varepsilon^{i},
$$

damos um critério para se determinar o valor crítico $\beta_{i}=\beta_{c}^{[i]}(d)$ na $i$-ésima ordem.

Uma contribuição técnica muito importante deste trabalho diz respeito ao controle de crescimento do módulo de derivadas de ordem superior de composições utilizando indução: aqui reunimos duas ferramentas pouco conhecidas, a saber,

(a) os coeficientes $C_{l}=A l !^{\lambda} / l^{2}$, que satisfazem (3.3.3) e (3.3.4) (aprendemos em [50])

(b) a fórmula de Scott (3.3.15) para a derivada de ordem superior de composições (aprendemos em [18]),

para formar uma ferramenta inteiramente nova e bastante poderosa. O item (a), por satisfazer as relações citadas, é adequado para controlar convoluções; devemos enfatizar que nessa tecnologia o fator $1 / l^{2}$ é de fundamental importância. Com respeito ao item (b), esse se mostra adequado ao uso do item (a) por apresentar um termo de convoluções, diferentemente de outras fórmulas para a derivada de ordem superior de composições, como por exemplo a fórmula de Faà di Bruno.

Encontram-se no Capítulo 6 as conclusões deste trabalho, bem como os comentários finais e o apontamento dos problemas em aberto. 


\section{Capítulo 2}

\section{Trajetória Discreta: Resumo}

\subsection{O Modelo $O(N)$ de Heisenberg Hierárquico}

Por um postulado devido a Gibbs, a termodinâmica de um sistema de spins é derivada de uma distribuição de probabilidade definida sobre o espaço de configuração, denominada medida de equilíbrio ou medida de Gibbs.

Para o modelo $O(N)$ de Heisenberg ferromagnético considerado sobre uma rede finita $\Lambda_{K} \subset \mathbb{Z}^{d}$ de cardinalidade $\left|\Lambda_{K}\right|=n$ essa medida é dada por

$$
d \nu_{n}^{(N)}(\boldsymbol{y})=\frac{1}{Z_{n}^{(N)}} \exp \left\{-\frac{1}{2}(\boldsymbol{y}, A \boldsymbol{y})_{\Omega_{n}}\right\} \prod_{i \in \Lambda_{K}} d \sigma_{0}^{(N)}\left(y_{i}\right)
$$

Nesta expressão,

$$
Z_{n}^{(N)}=\int_{\Omega_{n}} \exp \left\{-\frac{1}{2}(\boldsymbol{y}, A \boldsymbol{y})_{\Omega_{n}}\right\} \prod_{i \in \Lambda_{K}} d \sigma_{0}^{(N)}\left(y_{i}\right)
$$

é a chamada função de partição, uma normalização necessária para que $d \nu_{n}^{(N)}(\boldsymbol{y})$ seja uma medida de probabilidade; $\boldsymbol{y}$ denota um elemento do espaço de configuração $\Omega_{n}=$ $\mathbb{R}^{N} \times \cdots \times \mathbb{R}^{N}=\mathbb{R}^{N . n} ;(\boldsymbol{x}, \boldsymbol{y})_{\Omega_{n}}$ é o produto interno sobre $\Omega_{n}$

$$
(\boldsymbol{x}, \boldsymbol{y})_{\Omega_{n}}=\sum_{\boldsymbol{i} \in \Lambda_{K}} x_{\boldsymbol{i}} \cdot y_{\boldsymbol{i}}=\sum_{\boldsymbol{i} \in \Lambda_{K}} \sum_{q=1}^{N} x_{i}^{(q)} y_{\boldsymbol{i}}^{(q)}
$$




$$
d \sigma_{0}^{(N)}(y)=\frac{1}{S_{\beta N}^{N}} \delta(|y|-\sqrt{\beta N}) d^{N} y
$$

é a medida "a priori" uniforme sobre a esfera $N$-dimensional

$$
\Sigma_{\beta N}^{N}=\left\{y \in \mathbb{R}^{N}:|y|^{2}=\sum_{q=1}^{N}\left(y^{(q)}\right)^{2}=\beta N\right\}
$$

de raio $\sqrt{\beta N}$, com $\beta$ o inverso da temperatura e

$$
S_{\beta N}^{N}=\int_{\mathbb{R}^{N}} \delta(|y|-\sqrt{\beta N}) d^{N} y=\frac{2 \pi^{N / 2}(\sqrt{\beta N})^{N-1}}{\Gamma(N / 2)}
$$

a área da superfície da esfera $\Sigma_{\beta N}^{N}$. No modelo $O(N)$ de Heisenberg associamos a cada vértice $\boldsymbol{i} \in \Lambda_{K}$ uma variável de spin clássica $y_{\boldsymbol{i}}$ que assume valores sobre a esfera unitária em $\mathbb{R}^{N}$. Entretanto, para os propósitos de nosso estudo, é conveniente que se tenha $y_{\boldsymbol{i}} \in \mathbb{R}^{N}$. Assim sendo introduzimos, na medida "a priori", o vínculo (2.1.5). Também por conveniência escolhemos o raio $\sqrt{\beta N^{1}}$ ao raio unitário. Quanto ao acoplamento entre as variáveis de spin $y_{i}$, este é expresso pela matriz $A=\left[A_{i j}\right]$ presente na energia

$$
\mathcal{U}_{n}(\boldsymbol{y})=(\boldsymbol{y}, A \boldsymbol{y})_{\Omega_{n}}
$$

associada à configuração $\boldsymbol{y}$, sendo $A=-J \otimes I_{N} \operatorname{com} I_{N}$ a matriz identidade de ordem $N$ e $J=\left[J_{\boldsymbol{i}}\right]$ uma matriz $n \times n$ de interação ferromagnética:

$$
J_{i j} \geq 0 \quad \forall \boldsymbol{i}, \boldsymbol{j} \in \Lambda_{K}
$$

O nosso interesse está na chamada interação hierárquica, que será definida a seguir.

Por uma questão de completeza, façamos:

\footnotetext{
${ }^{1}$ Note que pela mudança de variável $\boldsymbol{y} \rightarrow \sqrt{\beta} \boldsymbol{y}$ o parâmetro $\beta$ passa a ser escrito no termo exponencial de (2.1.1) e não mais na medida "a priori" - perceba que $d \sigma_{0}^{(N)}(\sqrt{\beta} y)=\left.d \sigma_{0}^{(N)}(y)\right|_{\beta=1}$.
} 


\section{Cálculo da Área da Superfície de uma Esfera $N$-dimensional}

(a) de Raio Unitário: é conveniente que se passe para coordenadas esféricas generalizadas; o jacobiano é dado por

$$
d^{N} y=r^{N-1}\left(\sin \theta_{1}\right)^{N-2}\left(\sin \theta_{2}\right)^{N-3} \cdots \sin \theta_{N-2} d r d \theta_{1} d \theta_{2} \cdots d \theta_{N-1}
$$

$\operatorname{com} 0 \leq r=|y|<\infty$,

$$
0 \leq \theta_{1}, \theta_{2}, \ldots, \theta_{N-2} \leq \pi
$$

e

$$
0 \leq \theta_{N-1} \leq 2 \pi
$$

Para se chegar à igualdade

$$
\begin{aligned}
S_{1}^{N} & =\int_{0}^{\pi}\left(\sin \theta_{1}\right)^{N-2} d \theta_{1} \int_{0}^{\pi}\left(\sin \theta_{2}\right)^{N-3} d \theta_{2} \cdots \int_{0}^{\pi} \sin \theta_{N-2} d \theta_{N-2} \int_{0}^{2 \pi} d \theta_{N-1} \\
& =\frac{2 \pi^{N / 2}}{\Gamma(N / 2)}
\end{aligned}
$$

pode-se utilizar a integral gaussiana

$$
\int_{\mathbb{R}^{N}} e^{-|y|^{2}} d^{N} y=\left(\int_{\mathbb{R}} e^{-y^{2}} d y\right)^{N}=\pi^{N / 2}
$$

pois essa também é igual a

$$
\begin{aligned}
\int_{\mathbb{R}^{N}} e^{-|y|^{2}} d^{N} y & =S_{1}^{N} \int_{0}^{\infty} r^{N-1} e^{-r^{2}} d r \\
& =S_{1}^{N} \frac{1}{2} \int_{0}^{\infty} t^{N / 2-1} e^{-t} d t \\
& =S_{1}^{N} \frac{\Gamma(N / 2)}{2}
\end{aligned}
$$


(b) de Raio R: de (2.1.9) temos que

$$
S_{R^{2}}^{N}=R^{N-1} S_{1}^{N}
$$

de onde se conclui, juntamente com (2.1.10), a equação (2.1.6).

A Matriz de Interação Hierárquica. Dados os inteiros $L, K$ e $d, L, K>1$ e $d \geq 1$, seja para cada $m=1,2, \ldots, K$

$$
\Lambda_{m}=\left\{0,1, \ldots, L^{m}-1\right\}^{d} \subset \mathbb{Z}^{d}
$$

a rede finita hipercúbica $d$-dimensional de cardinalidade $\left|\Lambda_{m}\right|=L^{m d}$. Seja $u \in \mathbb{R}^{\left|\Lambda_{m}\right|}$ um vetor que associa a cada vértice $\boldsymbol{i}=\left(i_{1}, \ldots, i_{d}\right)$ de $\Lambda_{m}$ uma variável real $u_{\boldsymbol{i}}$, e $v \in \mathbb{R}^{\left|\Lambda_{m-1}\right|}$ um vetor que associa a cada vértice $\boldsymbol{r}=\left(r_{1}, \ldots, r_{d}\right)$ de $\Lambda_{m-1}$ uma variável real $v_{\boldsymbol{r}}$. Definimos o operador de bloco $B: \mathbb{R}^{\left|\Lambda_{m}\right|} \rightarrow \mathbb{R}^{\left|\Lambda_{m-1}\right|}$ por

$$
B u=v
$$

tal que a componente $\boldsymbol{r}$ desse vetor é dada por

$$
v_{\boldsymbol{r}}=(B u)_{\boldsymbol{r}}=\frac{1}{L^{d / 2}} \sum_{\boldsymbol{j} \in \Lambda_{1}} u_{L \boldsymbol{r}+\boldsymbol{j}}
$$

Seu adjunto $B^{*}: \mathbb{R}^{\left|\Lambda_{m-1}\right|} \rightarrow \mathbb{R}^{\left|\Lambda_{m}\right|}$

$$
(w, B u)_{\mathbb{R}^{\left|\Lambda_{m-1}\right|}}=\left(B^{*} w, u\right)_{\mathbb{R}^{\left|\Lambda_{m}\right|}}
$$

com respeito ao produto interno $(w, u)_{\mathbb{R}^{\left|\Lambda_{m}\right|}}=\sum_{\boldsymbol{i} \in \Lambda_{m}} w_{\boldsymbol{i}} u_{\boldsymbol{i}}$ é

$$
B^{*} v=u
$$

cuja componente $\boldsymbol{i}=L \boldsymbol{r}+\boldsymbol{j}, \boldsymbol{r} \in \Lambda_{m-1}$ e $\boldsymbol{j} \in \Lambda_{1}$, é dada por 


$$
u_{L \boldsymbol{r}+\boldsymbol{j}}=\left(B^{*} v\right)_{L \boldsymbol{r}+\boldsymbol{j}}=\frac{1}{L^{d / 2}} v_{\boldsymbol{r}} \quad \forall \boldsymbol{j} \in \Lambda_{1}
$$

Introduzimos assim as noções de bloco e hierarquia à rede $\Lambda_{K}$ : a cada vértice $\boldsymbol{r}$ de $\Lambda_{K-k-1}$ associamos um bloco de $L^{d}$ vértices de $\Lambda_{K-k}$, a saber, todos os elementos de

$$
\left\{L r_{1}, \ldots, L r_{1}+L-1\right\} \times \cdots \times\left\{L r_{d}, \ldots, L r_{d}+L-1\right\}
$$

os vértices $\boldsymbol{i} \in \Lambda_{K-k}$ são denominados vértices da hierarquia $k$ e os $\boldsymbol{r} \in \Lambda_{K-k-1}$ vértices $d a k+1$-ésima hierarquia; $\boldsymbol{i} \in \Lambda_{K}$ são vértices da hierarquia zero, e na $K$-ésima hierarquia há somente um único vértice $\Lambda_{0}=\{\mathbf{0}\}$. Se $u$ e $v$ denotam configurações de spins, dado um vértice $\boldsymbol{r}$ da $k+$ 1-ésima hierarquia, a operação realizada pelo operador $B$ é a de somar todas as variáveis de spin $u_{\boldsymbol{i}}$ da hierarquia $k$ associadas a $\boldsymbol{r}$, e normalizar pela raiz quadrada do tamanho do bloco $\left(L^{d}\right)$. Quanto a $B^{*}$, transforma a variável de spin de bloco $v_{\boldsymbol{r}}$ da $k+1$-ésima hierarquia em $L^{d}$ variáveis de spin da hierarquia $k$, atribuindo a cada uma delas o mesmo valor.

Utilizando os operadores $B$ e $B^{*}$ definimos, sobre $\mathbb{R}^{\left|\Lambda_{K}\right|}$, a matriz de interação hierárquica $J_{H}$ :

$$
J_{H}=\sum_{k=1}^{K} L^{-2 k}\left(B^{*}\right)^{k} B^{k}
$$

$B^{k}=\underbrace{B B \cdots B}_{k \text {-vezes }}$ é a aplicação sucessiva de $B$, e portanto $B^{k}: \mathbb{R}^{\left|\Lambda_{K}\right|} \rightarrow \mathbb{R}^{\left|\Lambda_{K-k}\right|} ;$ o índice $k$ indica a hierarquia. Assim, o número máximo de hierarquias que se tem em $\Lambda_{K}$ é $K$.

Definição 2.1.1 (Matriz Positiva e Positiva Definida) Uma matriz $M=\left[M_{i j}\right]$ $n \times n$ real é dita ser positiva se

$$
M_{i j}>0 \quad \forall i, j
$$

e é dita ser positiva definida se 


$$
(u, M u)_{\mathbb{R}^{n}}>0
$$

para todo vetor não nulo $u \in \mathbb{R}^{n}$.

Proposição 2.1.2 $J_{H}$ é uma matriz $L^{K d} \times L^{K d}$ real simétrica positiva e positiva definida.

Para demonstrarmos esta proposição é necessária a definição de distância hierárquica: consideremos uma seqüência $\left(\boldsymbol{\theta}_{1}, \ldots, \boldsymbol{\theta}_{K}\right)$ de vetores $\boldsymbol{\theta}_{k} \in\{0,1, \ldots, L-1\}^{d}$ tal que, para cada vértice $\boldsymbol{i}=\left(i_{1}, \ldots, i_{d}\right) \in \Lambda_{K}$, temos uma única expansão na base $L^{d}$-nária

$$
\boldsymbol{i}=\sum_{k=1}^{K} \boldsymbol{\theta}_{k} L^{k-1} .
$$

O vetor $\boldsymbol{\theta}_{k}$ indica a posição do vértice $\boldsymbol{i}$ dentro do bloco, ao qual pertence, da $k$-ésima hierarquia. A título de esclarecimento, consideremos o exemplo mostrado pela Figura 2.1 , em que $L=2$ e $d=1$.

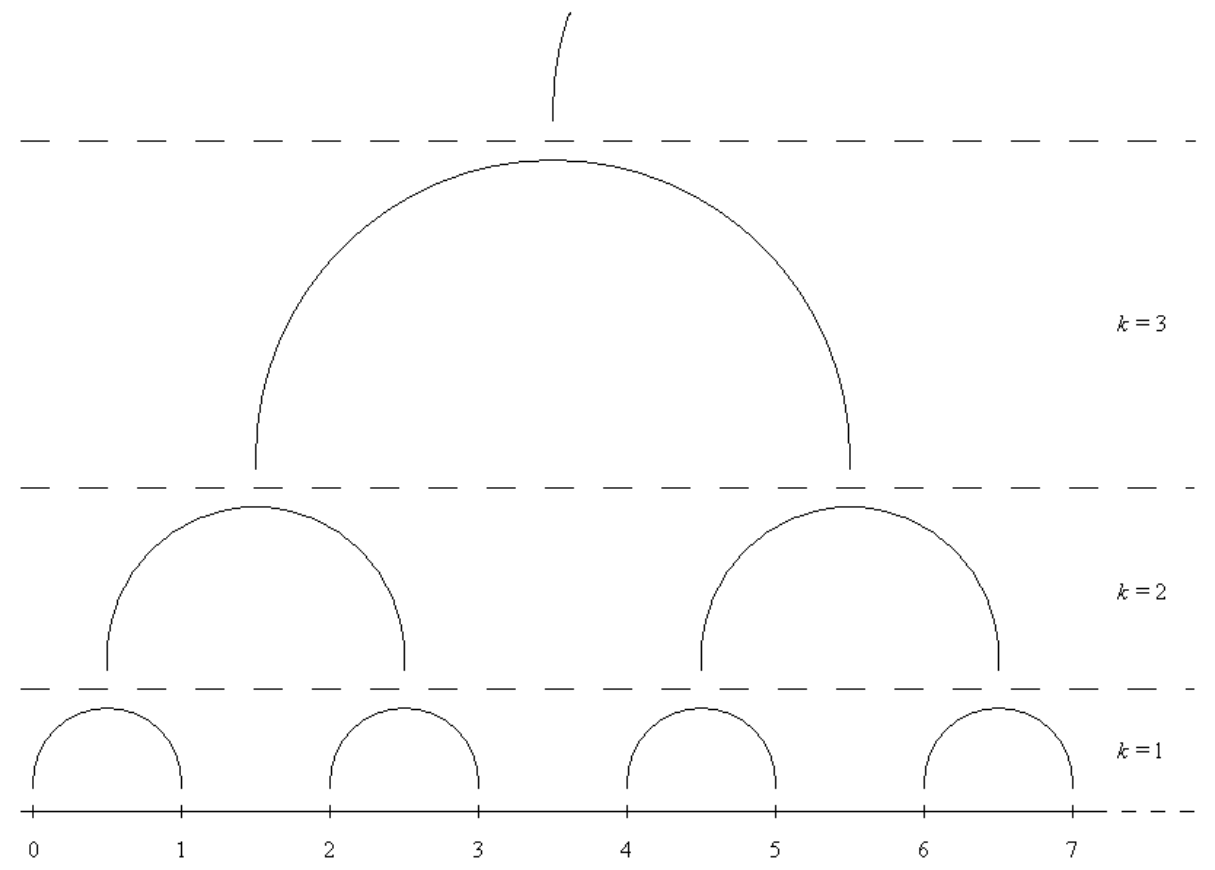

Figura 2.1: Esquema hierárquico da rede $\Lambda_{K}$ no caso em que $L=2$ e $d=1$. 
O vértice $i=3$ possui a expansão binária $(1,1,0,0, \ldots, 0)$ : dentro do bloco da primeira hierarquia ele ocupa a posição $\theta_{1}=1$; dentro do bloco da segunda hierarquia, a posição $\theta_{2}=1$; dentro do bloco da terceira hierarquia, a posição $\theta_{3}=0$; e assim sucessivamente. Ao vértice $i=4$ está associada a expansão $(0,0,1,0,0, \ldots, 0)$.

Definição 2.1.3 (Distância Hierárquica) Seja $\left(\boldsymbol{\theta}_{1}, \ldots, \boldsymbol{\theta}_{K}\right)$ a expansão $L^{d}$-nária do vértice $\boldsymbol{i} \in \Lambda_{K}, e\left(\boldsymbol{\theta}_{1}^{\prime}, \ldots, \boldsymbol{\theta}_{K}^{\prime}\right)$ a do vértice $\boldsymbol{j} \in \Lambda_{K}$. A distância hierárquica entre $\boldsymbol{i}$ e $\boldsymbol{j}$ é definida por

$$
\operatorname{dist}_{L}(\boldsymbol{i}, \boldsymbol{j})= \begin{cases}L^{k(\boldsymbol{i}, \boldsymbol{j})} & \text { se } \boldsymbol{i} \neq \boldsymbol{j} \\ 0 & \text { se } \boldsymbol{i}=\boldsymbol{j}\end{cases}
$$

sendo $k(\boldsymbol{i}, \boldsymbol{j})=\max \left\{l \in\{0,1, \ldots, K\}: \boldsymbol{\theta}_{l} \neq \boldsymbol{\theta}_{l}^{\prime}\right\}$ a menor hierarquia $k$ que faz com que $\boldsymbol{i}$ e $\boldsymbol{j}$ sejam cobertos por um mesmo bloco de tamanho $L^{k d}$.

Peguemos como exemplo os sítios 3 e 4 da Figura 2.1, cujas expansões binárias já conhecemos: $k(3,4)=3$; logo $\operatorname{dist}_{2}(3,4)=2^{3}=8$, apesar de serem vizinhos próximos.

Observação 2.1.4 Note que a distância hierárquica não é estacionária com respeito a qualquer translação $\boldsymbol{a} \in \mathbb{Z}^{d}$, isto é, em geral dist ${ }_{L}(\boldsymbol{i}+\boldsymbol{a}, \boldsymbol{j}+\boldsymbol{a}) \neq \operatorname{dist}_{L}(\boldsymbol{i}, \boldsymbol{j})$. Além disso, a desigualdade $\operatorname{dist}_{L}(\boldsymbol{i}, \boldsymbol{j})>|\boldsymbol{i}-\boldsymbol{j}|$ é sempre satisfeita, com $|\boldsymbol{i}-\boldsymbol{j}|$ a distância euclidiana entre os sítios.

Estamos agora em posição de demonstrar a Proposição 2.1.2:

Demonstração. Seja $\delta_{\boldsymbol{i}} \in \mathbb{R}^{\left|\Lambda_{K}\right|}$ um vetor cujas componentes $u_{\boldsymbol{l}}$ são nulas exceto para $\boldsymbol{l}=\boldsymbol{i}, \operatorname{com} \boldsymbol{l}, \boldsymbol{i} \in \Lambda_{K}$ :

$$
\left(\delta_{i}\right)_{\boldsymbol{l}}=\left\{\begin{array}{lll}
0 & \text { se } & \boldsymbol{l} \neq \boldsymbol{i} \\
1 & \text { se } & \boldsymbol{l}=\boldsymbol{i}
\end{array} .\right.
$$

O elemento de matriz $\left(J_{H}\right)_{i j}$ é dado por 


$$
\begin{aligned}
\left(J_{H}\right)_{\boldsymbol{i j}} & =\left(\delta_{\boldsymbol{i}}, J_{H} \delta_{\boldsymbol{j}}\right)_{\mathbb{R}^{\left|\Lambda_{K}\right|}} \\
& =\sum_{k=1}^{K} L^{-2 k}\left(\delta_{\boldsymbol{i}},\left(B^{*}\right)^{k} B^{k} \delta_{\boldsymbol{j}}\right)_{\mathbb{R}^{\mid \Lambda_{K}} \mid} \\
& =\sum_{k=1}^{K} L^{-2 k}\left(B^{k} \delta_{\boldsymbol{i}}, B^{k} \delta_{\boldsymbol{j}}\right)_{\mathbb{R}^{\left|\Lambda_{K-k}\right|}}
\end{aligned}
$$

Para $i \neq j$

$$
\left(B^{k} \delta_{\boldsymbol{i}}, B^{k} \delta_{\boldsymbol{j}}\right)_{\mathbb{R}^{\left|\Lambda_{K-k}\right|}}=\left\{\begin{array}{ll}
0 & \text { se } \quad k<k(\boldsymbol{i}, \boldsymbol{j}) \\
L^{-d k} & \text { se } \quad k \geq k(\boldsymbol{i}, \boldsymbol{j})
\end{array},\right.
$$

e para $\boldsymbol{i}=\boldsymbol{j}$ temos que $\left(B^{k} \delta_{\boldsymbol{i}}, B^{k} \delta_{\boldsymbol{j}}\right)_{\mathbb{R}^{\left|\Lambda_{K-k}\right|}}=L^{-d k}$ para todo $k$. Desta maneira

$$
\left(J_{H}\right)_{\boldsymbol{i} \boldsymbol{j}}=\left\{\begin{array}{ll}
\left(L^{-(d+2) k(\boldsymbol{i}, \boldsymbol{j})}-L^{-(d+2)(K+1)}\right)\left(1-L^{-(d+2)}\right)^{-1} & \text { se } \boldsymbol{i} \neq \boldsymbol{j} \\
\left(L^{-(d+2)}-L^{-(d+2)(K+1)}\right)\left(1-L^{-(d+2)}\right)^{-1} & \text { se } \boldsymbol{i}=\boldsymbol{j}
\end{array} .\right.
$$

Uma vez que $k(\boldsymbol{i}, \boldsymbol{j})=k(\boldsymbol{j}, \boldsymbol{i})$, e $\left(J_{H}\right)_{\boldsymbol{i} \boldsymbol{j}}>0$ e real para quaisquer $\boldsymbol{i}$ e $\boldsymbol{j}$ da rede $\Lambda_{K}$, temos que $J_{H}$ é uma matriz real simétrica positiva.

Seja agora $s \in \mathbb{R}^{\left|\Lambda_{K}\right|}$ um vetor qualquer não nulo e que associa a cada vértice $\boldsymbol{i}$ de $\Lambda_{K}$ uma variável real $s_{\boldsymbol{i}}$, e $\boldsymbol{r}=\left(r_{1}, \ldots, r_{d}\right)$. Então a forma quadrática associada a $J_{H}$ é

$$
\begin{aligned}
\left(s, J_{H} s\right)_{\mathbb{R}^{\left|\Lambda_{K}\right|}} & =\sum_{k=1}^{K} L^{-2 k}\left(s,\left(B^{*}\right)^{k} B^{k} s\right)_{\mathbb{R}^{\left|\Lambda_{K}\right|}} \\
& =\sum_{k=1}^{K} L^{-2 k}\left(B^{k} s, B^{k} s\right)_{\mathbb{R}^{\left|\Lambda_{K-k}\right|}} \\
& =\sum_{k=1}^{K} L^{-2 k} \sum_{\boldsymbol{r} \in \Lambda_{K-k}}\left(B^{k} s\right)_{\boldsymbol{r}}^{2}>0
\end{aligned}
$$

com 


$$
\left(B^{k} s\right)_{\boldsymbol{r}}=\frac{1}{L^{d k / 2}} \sum_{\boldsymbol{j} \in \Lambda_{k}} s_{L^{k}} \boldsymbol{r}+\boldsymbol{j}
$$

Assim, $J_{H}$ é uma matriz positiva definida.

Pela Proposição 2.1.2 a matriz de interação hierárquica $J_{H}$, definida por (2.1.21), é uma matriz de interação ferromagnética (vide (2.1.8)). Em vista desse fato, temos:

Definição 2.1.5 O modelo $O(N)$ de Heisenberg hierárquico é definido pela medida de Gibbs (2.1.1) com energia de interação ferromagnética

$$
\mathcal{U}_{n}(\boldsymbol{y})=(\boldsymbol{y}, A \boldsymbol{y})_{\Omega_{n}}=-(L-1)\left(\boldsymbol{y},\left(J_{H} \otimes I_{N}\right) \boldsymbol{y}\right)_{\Omega_{n}}
$$

sendo $n=\left|\Lambda_{K}\right|=L^{K d}$. O fator $(L-1)$ é acrescentado para garantir, no limite em que $L \downarrow 1$, a convergência do laplaceano hierárquico para o laplaceano hierárquico contínuo, como pode ser visto em [8].

\subsection{Transformação de Grupo de Renormalização}

Comecemos esta seção por notar a estrutura da energia hierárquica $\mathcal{U}_{L^{K d}}(\boldsymbol{y})$ :

$$
\begin{aligned}
\mathcal{U}_{L^{K d}}(\boldsymbol{y}) & =-(L-1) \sum_{m=1}^{K} L^{-2 m}\left(\left(B^{m} \otimes I_{N}\right) \boldsymbol{y},\left(B^{m} \otimes I_{N}\right) \boldsymbol{y}\right)_{\Omega_{L^{(K-m) d}}} \\
& =L^{-2} \mathcal{U}_{L^{(K-1) d}}\left(\boldsymbol{y}^{[1]}\right)-(L-1) L^{-2} \sum_{\boldsymbol{r} \in \Lambda_{K-1}}\left|y_{\boldsymbol{r}}^{[1]}\right|^{2}
\end{aligned}
$$

que é a energia hierárquica normalizada por $L^{-2}$ de uma configuração de spins de bloco $\boldsymbol{y}^{[1]}=\left(B \otimes I_{N}\right) \boldsymbol{y}$ da primeira hierarquia sobre a rede $\Lambda_{K-1}$, somada à energia de interação das $L^{(K-1) d}$ variáveis de spin de bloco $y_{\boldsymbol{r}}^{[1]}=\left(\left(B \otimes I_{N}\right) \boldsymbol{y}\right)_{\boldsymbol{r}}$ da primeira hierarquia. De maneira mais geral 


$$
\mathcal{U}_{L^{K d}}(\boldsymbol{y})=L^{-2 k} \mathcal{U}_{L^{(K-k) d}}\left(\boldsymbol{y}^{[k]}\right)-(L-1) L^{-2 k} \sum_{\boldsymbol{r} \in \Lambda_{K-k}}\left|y_{\boldsymbol{r}}^{[k]}\right|^{2}
$$

com $\boldsymbol{y}^{[k]}=\left(B^{k} \otimes I_{N}\right) \boldsymbol{y}$ a configuração de spins de bloco da $k$-ésima hierarquia. É justamente esta característica da energia hierárquica que permite a implementação do grupo de renormalização mais facilmente.

Relação de Recorrência. O nosso interesse está na evolução, com o aumento da hierarquia $k$, da distribuição da variável soma

$$
X_{k, N}^{\gamma}=\frac{1}{\sqrt{m^{\gamma / d}}} \sum_{i \in \Lambda_{k}} y_{i}
$$

com $m=\left|\Lambda_{k}\right|=L^{k d}$. Definimos com essa finalidade a medida "a priori" $\sigma_{k}^{(N)}$ associada à escala $k$ pela seguinte equação:

$$
\begin{aligned}
& \int_{\Omega_{L} K d} \delta\left(L^{-\left(\frac{\gamma-d}{2}\right) k}\left(B^{k} \otimes I_{N}\right) \boldsymbol{y}^{\prime}-\boldsymbol{y}\right) d \nu_{L^{K d}}^{(N)}\left(\boldsymbol{y}^{\prime}\right)= \\
& =\frac{1}{Z_{L^{(K-k) d}}^{(N)}} \exp \left\{-\frac{c_{\gamma, k}}{2} \mathcal{U}_{L^{(K-k) d}}(\boldsymbol{y})\right\} \prod_{\boldsymbol{i} \in \Lambda_{K-k}} d \sigma_{k}^{(N)}\left(y_{i}\right)
\end{aligned}
$$

integramos a medida (2.1.1) sobre o espaço de configuração mantendo a configuração de spins de bloco da $k$-ésima hierarquia fixa - (2.2.4) é uma medida marginal sobre $\Omega_{L^{(K-k) d}}$. O fator $\gamma$ pode assumir o valor $d+2$ ou $d$, introduzido a fim de se incluir ou não a normalização $L^{-k}$, presente em (2.2.2), à variável de bloco; assim,

$$
c_{\gamma, k}=\left\{\begin{array}{lll}
1 & \text { se } & \gamma=d+2 \\
L^{-2 k} & \text { se } & \gamma=d
\end{array} .\right.
$$

Quando $\gamma=d+2$ diz-se que a normalização é anormal, o que na verdade expressa uma normalização mais que normal devido ao fator adicional 2 em relação à normalização dita normal, caso em que $\gamma=d$, como é feito no Teorema Central do Limite usual. 
Estabeleceremos agora uma relação recursiva para as medidas "a priori":

Proposição 2.2.1 As medidas "a priori" associadas a escalas consecutivas relacionamse por

$$
\sigma_{k}^{(N)}(y)=\frac{1}{C_{k}} e^{c_{\gamma, k}(L-1)|y|^{2} / 2} \underbrace{\sigma_{k-1}^{(N)} * \cdots * \sigma_{k-1}^{(N)}}_{L^{d}-\text { termos }}\left(L^{\gamma / 2} y\right)
$$

com condição inicial

$$
\sigma_{0}^{(N)}(y)=\left\{\begin{array}{lll}
0 & \text { se } & |y|<\sqrt{\beta N} \\
1 & \text { se } & |y| \geq \sqrt{\beta N}
\end{array}\right.
$$

sendo que * denota o produto de convolução

$$
\rho * \eta(y)=\int_{\mathbb{R}^{N}} \rho\left(y-y^{\prime}\right) d \eta\left(y^{\prime}\right)
$$

e $C_{k}$ uma normalização que garante que $\sigma_{k}^{(N)}$ é uma medida de probabilidade.

Demonstração. Sendo (2.2.4) uma medida marginal, podemos obtê-la pela integração da medida de Gibbs

$$
\frac{1}{Z_{L^{(K-(k-1)) d}}^{(N)}} \exp \left\{-\frac{c_{\gamma, k-1}}{2} \mathcal{U}_{L^{(K-(k-1)) d}}\left(\boldsymbol{y}^{\prime}\right)\right\} \prod_{\boldsymbol{i} \in \Lambda_{K-(k-1)}} d \sigma_{k-1}^{(N)}\left(y_{\boldsymbol{i}}^{\prime}\right)
$$

sobre o espaço de configuração $\Omega_{L^{(K-(k-1)) d}}$ mantendo-se fixa a configuração de spins de bloco normalizada $L^{-\left(\frac{\gamma-d}{2}\right)}\left(B \otimes I_{N}\right) \boldsymbol{y}^{\prime} \in \Omega_{L^{(K-k) d}}$. Para isso precisamos de uma relação entre as energias $\mathcal{U}_{L^{(K-(k-1)) d}}\left(\boldsymbol{y}^{\prime}\right)$ e $\mathcal{U}_{L^{(K-k) d}}\left(\left(B \otimes I_{N}\right) \boldsymbol{y}^{\prime}\right)$ análoga à $(2.2 .1) ;$ trocando $K$ por $K-(k-1)$ em $(2.2 .1)$ :

$$
\begin{aligned}
\mathcal{U}_{L^{(K-(k-1)) d}}\left(\boldsymbol{y}^{\prime}\right)= & L^{-2} \mathcal{U}_{L^{(K-k) d}}\left(\left(B \otimes I_{N}\right) \boldsymbol{y}^{\prime}\right) \\
& -(L-1) L^{-2} \sum_{\boldsymbol{r} \in \Lambda_{K-k}}\left|\left(\left(B \otimes I_{N}\right) \boldsymbol{y}^{\prime}\right)_{\boldsymbol{r}}\right|^{2}
\end{aligned}
$$


Note que

$$
\begin{aligned}
L^{-\left(\frac{\gamma-d}{2}\right)}\left(\left(B \otimes I_{N}\right) \boldsymbol{y}^{\prime}\right)_{\boldsymbol{r}} & =\frac{1}{L^{\left(\frac{\gamma-d}{2}\right)}} \frac{1}{L^{d / 2}} \sum_{\boldsymbol{j} \in \Lambda_{1}} y_{L \boldsymbol{r}+\boldsymbol{j}}^{\prime} \\
& =\frac{1}{L^{\gamma / 2}} \sum_{\boldsymbol{j} \in \Lambda_{1}} y_{L \boldsymbol{r}+\boldsymbol{j}}^{\prime}
\end{aligned}
$$

Utilizando a seguinte decomposição e notação

$$
\begin{aligned}
\delta\left(L^{-\left(\frac{\gamma-d}{2}\right)}\left(B \otimes I_{N}\right) \boldsymbol{y}^{\prime}-\boldsymbol{y}\right) & =\prod_{\boldsymbol{r} \in \Lambda_{K-k}} \delta\left(L^{-\left(\frac{\gamma-d}{2}\right)}\left(\left(B \otimes I_{N}\right) \boldsymbol{y}^{\prime}\right)_{\boldsymbol{r}}-y_{\boldsymbol{r}}\right) \\
& =\prod_{\boldsymbol{r} \in \Lambda_{K-k}} \delta(\cdot \boldsymbol{r}),
\end{aligned}
$$

temos então

$$
\begin{aligned}
& \int_{\Omega_{L^{(K-(k-1)) d}}} \delta\left(L^{-\left(\frac{\gamma-d}{2}\right)}\left(B \otimes I_{N}\right) \boldsymbol{y}^{\prime}-\boldsymbol{y}\right) \times(2.2 .9)= \\
& =\int_{\Omega_{L}(K-k) d} \int_{\Omega_{L^{d}}} \prod_{\boldsymbol{r} \in \Lambda_{K-k}}\left\{e^{c_{\gamma, k-1}(L-1) L^{-2}\left|\left(\left(B \otimes I_{N}\right) \boldsymbol{y}^{\prime}\right) \boldsymbol{r}\right|^{2} / 2} \delta(\cdot \boldsymbol{r}) \prod_{\boldsymbol{j} \in \Lambda_{1}} d \sigma_{k-1}^{(N)}\left(y_{L \boldsymbol{r}+\boldsymbol{j}}^{\prime}\right)\right\} \times \\
& \times \frac{1}{Z_{L^{(K-(k-1)) d}}^{(N)}} \exp \left\{-\frac{c_{\gamma, k-1} L^{-2}}{2} \mathcal{U}_{L^{(K-k) d}}\left(\left(B \otimes I_{N}\right) \boldsymbol{y}^{\prime}\right)\right\} \\
& =\frac{1}{Z_{L^{(K-k) d}}^{(N)}} \exp \left\{-\frac{c_{\gamma, k}}{2} \mathcal{U}_{L^{(K-k) d}}(\boldsymbol{y})\right\} \prod_{\boldsymbol{r} \in \Lambda_{K-k}} \frac{1}{C_{k}} e^{c_{\gamma, k}(L-1)\left|y_{\boldsymbol{r}}\right|^{2} / 2} d(\underbrace{\sigma_{k-1}^{(N)} * \cdots * \sigma_{k-1}^{(N)}}_{L^{d}-\text { termos }})\left(L^{\gamma / 2} y_{\boldsymbol{r}}\right) ;
\end{aligned}
$$

$L^{d}$ termos porque a cardinalidade de $\Lambda_{1}$ é $L^{d}$. Para concluirmos (2.2.6) devemos comparar (2.2.13) com (2.2.4). 
Quanto à condição inicial (2.2.7), essa é a função distribuição

$$
\int_{-\infty}^{y^{(1)}} \cdots \int_{-\infty}^{y^{(N)}} d \sigma_{0}^{(N)}\left(y^{\prime}\right) \quad y^{\prime} \in \mathbb{R}^{N}
$$

associada à medida "a priori" inicial (2.1.4).

Observação 2.2.2 Pela mudança de variável $\boldsymbol{y} \rightarrow \sqrt{\beta} \boldsymbol{y}$ em (2.1.1) (lembrar da nota de rodapé 1, página 32), (2.2.6) passa a ser escrita

$$
\sigma_{k}^{(N)}(y)=\frac{1}{C_{k}} e^{\beta c_{\gamma, k}(L-1)|y|^{2} / 2} \underbrace{\sigma_{k-1}^{(N)} * \cdots * \sigma_{k-1}^{(N)}}_{L^{d}-\text { termos }}\left(L^{\gamma / 2} y\right)
$$

com condição inicial

$$
\sigma_{0}^{(N)}(y)=\left\{\begin{array}{lll}
0 & \text { se } & |y|<\sqrt{N} \\
1 & \text { se } & |y| \geq \sqrt{N}
\end{array}\right.
$$

No limite de altas temperaturas $\left(\beta \rightarrow 0^{+}\right)$temos apenas a convolução de $L^{d}$ medidas "a priori". Isto está em pleno acordo com o fato que nesse limite as variáveis de spin comportam-se como variáveis aleatórias independentes (não há acoplamento).

Observação 2.2.3 Se $\mathcal{M}$ denota o espaço das medidas "a priori" em $\mathbb{R}^{N}$ invariantes por transformações ortogonais $O(N)$, (2.2.6) define um mapa $\mathcal{R}: \mathcal{M} \rightarrow \mathcal{M}$

$$
\sigma_{k}^{(N)}=\mathcal{R} \sigma_{k-1}^{(N)}
$$

e a coleção $\left\{\sigma_{k}^{(N)}(y)\right\}_{k=0}^{K}$ uma trajetória discreta sobre esse espaço. 
Relação de Recorrência Dual. Consideremos agora a função característica da medida "a priori" associada à escala $k$

$$
\phi_{k}^{(N)}(z)=\int_{\mathbb{R}^{N}} \exp \{i z \cdot y\} d \sigma_{k}^{(N)}(y)
$$

Proposição 2.2.4 A medida "a priori" $\phi_{k}^{(N)}(z)$ obedece a relação de recorrência

$$
\phi_{k}^{(N)}(z)=\frac{1}{N_{k}} \exp \left\{-\frac{c_{\gamma, k}(L-1)}{2} \Delta\right\}\left(\phi_{k-1}^{(N)}\left(L^{-\gamma / 2} z\right)\right)^{L^{d}}
$$

com

$$
\phi_{0}^{(N)}(z)=\frac{\Gamma(N / 2)}{(\sqrt{\beta N}|z| / 2)^{N / 2-1}} J_{N / 2-1}(\sqrt{\beta N}|z|),
$$

sendo $\Delta=\partial^{2} / \partial z_{1}^{2}+\cdots+\partial^{2} / \partial z_{N}^{2}$ o operador laplaceano $N$-dimensional, $J_{\nu}(\xi)$ a função de Bessel de ordem $\nu, \Gamma(n)$ a função gama de Euler, e $N_{k}$ uma normalização tal que $\phi_{k}^{(N)}(0)=1$.

Demonstração. Por (2.2.6), ignorando o fator $C_{k}$, temos que

$$
\phi_{k}^{(N)}(z)=\int_{\mathbb{R}^{N}} \exp \{i z \cdot y\} e^{c_{\gamma, k}(L-1)|y|^{2} / 2} d(\underbrace{\sigma_{k-1}^{(N)} * \cdots * \sigma_{k-1}^{(N)}}_{L^{d}-\text { termos }})\left(L^{\gamma / 2} y\right) .
$$

Uma vez que o laplaceano atua somente na variável $z$

$$
\begin{aligned}
e^{i z \cdot y} e^{c_{\gamma, k}(L-1)|y|^{2} / 2} & =\sum_{n=0}^{\infty} \frac{1}{n !}\left(\frac{c_{\gamma, k}(L-1)}{2}\right)^{n}(|y|)^{2 n} e^{i z \cdot y} \\
& =\sum_{n=0}^{\infty} \frac{1}{n !}\left(\frac{c_{\gamma, k}(L-1)}{2}\right)^{n} \frac{(\Delta)^{n}}{\left(i^{2}\right)^{n}} e^{i z \cdot y} \\
& =\exp \left\{-\frac{c_{\gamma, k}(L-1)}{2} \Delta\right\} e^{i z \cdot y},
\end{aligned}
$$


e (2.2.18) fica

$$
\begin{aligned}
\phi_{k}^{(N)}(z) & =\exp \left\{-\frac{c_{\gamma, k}(L-1)}{2} \Delta\right\} \int_{\mathbb{R}^{N}} \exp \{i z \cdot y\} d(\underbrace{\sigma_{k-1}^{(N)} * \cdots * \sigma_{k-1}^{(N)}}_{L^{d}-\text { termos }})\left(L^{\gamma / 2} y\right) \\
& =\exp \left\{-\frac{c_{\gamma, k}(L-1)}{2} \Delta\right\} \int_{\mathbb{R}^{N}} \exp \left\{i L^{-\gamma / 2} z \cdot y\right\} d(\underbrace{\sigma_{k-1}^{(N)} * \cdots * \sigma_{k-1}^{(N)}}_{L^{d}-\text { termos }})(y) .
\end{aligned}
$$

Pelo Teorema da Convolução e pela definição (2.2.15), temos então que

$$
\phi_{k}^{(N)}(z)=\exp \left\{-\frac{c_{\gamma, k}(L-1)}{2} \Delta\right\}\left(\phi_{k-1}^{(N)}\left(L^{-\gamma / 2} z\right)\right)^{L^{d}}
$$

De $(2.2 .15)$ e do fato que $\sigma_{k}^{(N)}(y)$ é uma medida de probabilidade, vê-se a necessidade de que $\phi_{k}^{(N)}(0)=1$ para todo $k$ e todo $L$. Normalizamos assim (2.2.21) por

$$
N_{k}=\left.\exp \left\{-\frac{c_{\gamma, k}(L-1)}{2} \Delta\right\}\left(\phi_{k-1}^{(N)}\left(L^{-\gamma / 2} z\right)\right)^{L^{d}}\right|_{z=0}
$$

Para finalizarmos esta demonstração temos de fazer a transformada de Fourier da medida "a priori" inicial (2.2.7). Utilizando coordenadas esféricas generalizadas (lembrando que o jacobiano é dado por (2.1.9)), efetuando a mudança de variável $\theta=\theta_{1}-\pi / 2$, e fazendo uso da igualdade (2.1.10) com $N$ trocado por $N-1$, temos:

$$
\begin{aligned}
\phi_{0}^{(N)}(z) & =\frac{1}{S_{\beta N}^{N}} \int_{\mathbb{R}^{N}} \exp \{i z \cdot y\} \delta(|y|-\sqrt{\beta N}) d^{N} y \\
& =\frac{S_{1}^{N-1}}{S_{\beta N}^{N}} \int_{0}^{\infty} r^{N-1} \delta(r-\sqrt{\beta N}) \int_{-\pi / 2}^{\pi / 2} e^{-i|z| r \sin \theta}(\cos \theta)^{N-2} d \theta d r \\
& =\frac{2 \pi^{N / 2}}{S_{\beta N}^{N}} \int_{0}^{\infty} r^{N-1} \delta(r-\sqrt{\beta N}) \frac{J_{N / 2-1}(r|z|)}{(r|z| / 2)^{N / 2-1}} d r \\
& =\frac{\Gamma(N / 2)}{(\sqrt{\beta N}|z| / 2)^{N / 2-1}} J_{N / 2-1}(\sqrt{\beta N}|z|)
\end{aligned}
$$


sendo

$$
J_{\nu}(\xi)=\frac{(\xi / 2)^{\nu}}{\sqrt{\pi} \Gamma(\nu+1 / 2)} \int_{-\pi / 2}^{\pi / 2} \cos (\xi \sin \theta)(\cos \theta)^{2 \nu} d \theta
$$

com $\Re e(\nu)>-1 / 2$, a representação integral da função de Bessel de ordem $\nu$ (vide equação (20) do Capítulo VII de [10]). Note que

$$
\phi_{0}^{(N)}(0)=\frac{1}{S_{\beta N}} \int_{\mathbb{R}^{N}} \delta(|y|-\sqrt{\beta N}) d^{N} y=1
$$

não havendo necessidade de uma normalização.

Comentário 2.2.5 As relações recursivas (2.2.6) e (2.2.16) recebem o nome de transformação de grupo de renormalização.

Observação 2.2.6 Uma vez que $\phi_{0}^{(N)}(z)$ depende apenas do módulo $|z|=\sqrt{z \cdot z} d a$ variável e o operador laplaceano preserva simetria esférica, segue que $\phi_{k}^{(N)}(z)$ é uma função de $|z|$ para todo $k=1,2, \ldots, K$.

Teorema de Lee-Yang. Devemos ressaltar que o fato de termos feito a transformada de Fourier ${ }^{2}$ da medida "a priori" é de extrema importância, pois é através dessa que se estabelece a relação com o teorema de Lee-Yang.

Definição 2.2.7 (Classe $\mathcal{L}$ de Funções) A família $\mathcal{L}$ é formada por funções inteiras que possuem a representação

$$
f(x)=C x^{m} \exp (\lambda x) \prod_{n=1}^{\infty}\left(1+\frac{x}{\gamma_{n}}\right)
$$

com $C \in \mathbb{C}, m$ um inteiro não-negativo, $\lambda \geq 0$ e $\left\{-\gamma_{n}, n \geq 1\right\}$ números reais nãopositivos tais que $-\gamma_{n+1} \leq-\gamma_{n} \leq 0$ para todo $n=1,2,3, \ldots$ e $\sum_{n=1}^{\infty} \gamma_{n}^{-1}<\infty$.

\footnotetext{
${ }^{2}$ Em [30] e [32] os autores consideram a transformada de Laplace aos invés da transformada de Fourier. Todavia, como nos dois casos a função transformada é inteira, as conclusões não são afetadas.
} 
Os elementos de $\mathcal{L}$ são chamados de funções inteiras de Laguerre (vide [27]). Pelos trabalhos de Laguerre e Pólya (vide [27] ou [36]) sabe-se que $\mathcal{L}$ consiste de polinômios que possuem somente zeros reais não-positivos, bem como os limites uniformes desses polinômios sobre subconjuntos compactos de $\mathbb{C}$.

Definição 2.2.8 (Propriedade de Lee-Yang) Uma medida de probabilidade $\sigma$ em $\mathbb{R}^{N}$ invariante por transformações ortogonais $O(N)$ possui a propriedade de Lee-Yang se sua função característica $\phi(z)=\int_{\mathbb{R}^{N}} \exp \{i z \cdot y\} d \sigma(y)=\varphi(x)$, com $x=-|z|^{2}$, é tal que $\varphi(x)$ pertence a $\{f \in \mathcal{L}: f(0)=1\}$.

Apresentadas essas definições, enunciamos:

Proposição 2.2.9 A medida "a priori" $\sigma_{0}^{(N)}(y)$, dada por (2.2.7), possui a propriedade de Lee-Yang.

Demonstração. Por (2.2.15) (com $k=0)$ e (2.2.17)

$$
\begin{aligned}
\phi_{0}^{(N)}(z) & =\int_{\mathbb{R}^{N}} \exp \{i z \cdot y\} d \sigma_{0}^{(N)}(y) \\
& =\frac{\Gamma(N / 2)}{(i \sqrt{\beta N x} / 2)^{N / 2-1}} J_{N / 2-1}(i \sqrt{\beta N x})=\varphi_{0}^{(N)}(x),
\end{aligned}
$$

sendo $x=-|z|^{2}$.

Para mostrarmos que $\varphi_{0}^{(N)} \in \mathcal{L}$, lembremos que para $\nu>-1$ real a função de Bessel $J_{\nu}(\xi)$ possui infinitos zeros, todos reais (vide páginas 478 e 483 de [48]), sendo sua representação produto canônica dada por (vide Seção 15.41 de [48])

$$
J_{\nu}(\xi)=\frac{(\xi / 2)^{\nu}}{\Gamma(\nu+1)} \prod_{n=1}^{\infty}\left(1-\frac{\xi^{2}}{\alpha_{n, \nu}^{2}}\right)
$$

$\operatorname{com}\left\{\alpha_{n, \nu}, n \geq 1\right\}$ os zeros positivos de $J_{\nu}(\xi)$ (note que 0 e $\left\{-\alpha_{n, \nu}, n \geq 1\right\}$ são os outros zeros), indexados por $n$ de tal maneira que $0<\alpha_{n, \nu}<\alpha_{n+1, \nu}$ para todo $n=1,2,3, \ldots$. Logo

$$
\varphi_{0}^{(N)}(x)=\prod_{n=1}^{\infty}\left(1+\frac{x}{\frac{\alpha_{n, N / 2-1}^{2}}{\beta N}}\right)
$$


sendo que seus zeros

$$
\left\{-\frac{\alpha_{n, N / 2-1}^{2}}{\beta N}, n \geq 1\right\}
$$

são chamados de zeros de Lee-Yang. A condição $\sum_{n=1}^{\infty} \alpha_{n, N / 2-1}^{-2}<\infty$ é garantida pelo seguinte resultado:

Lema 2.2.10 $\sum_{n=1}^{\infty} \alpha_{n, \nu}^{-2}$ é convergente para qualquer $\nu>-1$ real.

Demonstração. Fixado $\nu$, para grandes argumentos $\xi>>\left|\nu^{2}-1 / 4\right|$ vale o comportamento assintótico

$$
J_{\nu}(\xi) \sim \sqrt{\frac{2}{\pi \xi}} \cos \left(\xi-\frac{\nu \pi}{2}-\frac{\pi}{4}\right)
$$

de modo que para $n$ suficientemente grande

$$
\alpha_{n, \nu} \sim \frac{(2 \nu+1) \pi}{4}+\frac{(2 n-1) \pi}{2}
$$

Não é muito difícil vermos que o lado direito dessa expressão é maior que $n \pi / 4$.

Agora, seja $n^{*}$ um inteiro suficientemente grande, tal que (2.2.32) valha. Então

$$
\begin{aligned}
\sum_{n=1}^{n^{*}} \frac{1}{\alpha_{n, \nu}^{2}}+\sum_{n=n^{*}+1}^{\infty} \frac{1}{\alpha_{n, \nu}^{2}} & <\sum_{n=1}^{n^{*}} \frac{1}{\alpha_{n, \nu}^{2}}+\frac{16}{\pi^{2}} \sum_{n=n^{*}+1}^{\infty} \frac{1}{n^{2}} \\
& <\sum_{n=1}^{n^{*}} \frac{1}{\alpha_{n, \nu}^{2}}+\frac{16}{\pi^{2}} \sum_{n=1}^{\infty} \frac{1}{n^{2}}<\infty
\end{aligned}
$$

haja vista que na primeira soma temos um número finito de termos, e que a segunda soma é igual a $\pi^{2} / 6$.

Conclui-se assim a demonstração da proposição. 
Espaço de Funções Inteiras e a Trajetória $\left\{\phi_{k}^{(N)}(z)\right\}_{k=1}^{\infty}$ • A abordagem apresentada nos trabalhos de Kozitsky ([30] e [32]) e Watanabe ([49]) envolve técnicas de grupo de renormalização, segundo as quais a convergência da trajetória $\left\{\sigma_{k}^{(N)}(y)\right\}_{k=1}^{\infty}$ de medidas "a priori" $\left(\sigma_{k}^{(N)}=\mathcal{R} \sigma_{k-1}^{(N)}, \mathcal{R}\right.$ dado por (2.2.6)) é investigada seguindo-se a trajetória $\left\{\phi_{k}^{(N)}(z)\right\}_{k=1}^{\infty}$ das funções características dessas medidas $\left(\phi_{k}^{(N)}=\mathcal{F} \phi_{k-1}^{(N)}, \mathcal{F}\right.$ dado por (2.2.16)). Apresentaremos neste tópico dois fatos importantes sobre o mapa $\mathcal{F}$, bem como a ferramenta que decorre de um deles. Também reproduziremos um trecho do artigo [32], tomando a liberdade de modificar as notações. Ao final exporemos as contribuições de [32] e [49], apontando a condição inicial $\phi_{0}^{(N)}(z)$ adotada em cada um.

Comecemos pelo seguinte fato:

Proposição 2.2.11 Para $\gamma=d+2$, a função

$$
\phi^{\text {gauss }}(z)=e^{-\frac{L+1}{2}|z|^{2}}
$$

é um ponto fixo do mapa (2.2.16), chamado de ponto fixo gaussiano ou trivial.

Demonstração. Por $(2.2 .5), c_{d+2, k}=1$; ignorando a normalização presente em (2.2.16), verificaremos que a relação de ponto fixo

$$
B e^{-A|z|^{2}}=\exp \left\{-\frac{L-1}{2} \Delta\right\}\left(B e^{-A L^{-(d+2)}|z|^{2}}\right)^{L^{d}}
$$

é satisfeita para constantes $A$ e $B$ devidamente escolhidas.

Fazendo uso das identidades

$$
e^{-C|z|^{2}}=\frac{1}{(4 C \pi)^{N / 2}} \int_{\mathbb{R}^{N}} e^{i z \cdot y} e^{-\frac{1}{4 C}|y|^{2}} d^{N} y
$$

e (2.2.19), temos que o lado direito de (2.2.35) é igual a 


$$
\begin{aligned}
& =\exp \left\{-\frac{L-1}{2} \Delta\right\} B^{L^{d}}\left(\frac{L^{2}}{4 A \pi}\right)^{N / 2} \int_{\mathbb{R}^{N}} e^{i z \cdot y} e^{-\frac{L^{2}}{4 A}|y|^{2}} d^{N} y \\
& =B^{L^{d}}\left(\frac{L^{2}}{4 A \pi}\right)^{N / 2} \int_{\mathbb{R}^{N}} e^{i z \cdot y} e^{-\left(\frac{L^{2}}{4 A}-\frac{L-1}{2}\right)|y|^{2}} d^{N} y \\
& =B^{L^{d}}\left(\frac{L^{2}}{4 A \pi}\right)^{N / 2}\left(\frac{4 A \pi}{L^{2}-2 A(L-1)}\right)^{N / 2} e^{-\frac{A}{L^{2}-2 A(L-1)}|z|^{2}} \\
& =B^{L^{d}}\left(\frac{L^{2}}{L^{2}-2 A(L-1)}\right)^{N / 2} e^{-\frac{A}{L^{2}-2 A(L-1)}|z|^{2}} .
\end{aligned}
$$

Da igualdade entre essa e o lado esquerdo de (2.2.35) segue que

$$
A=\frac{L+1}{2} \quad \text { e } \quad B=L^{-N /\left(L^{d}-1\right)} .
$$

A expressão (2.2.34) é encontrada se considerarmos que em (2.2.16) existe uma normalização que garante que $\phi^{\text {gauss }}(0)=1$.

Outro fato importante sobre (2.2.16) segue do teorema abaixo enunciado (vide referência [29]):

Teorema 2.2.12 Seja $L^{d}$ um inteiro e $\chi$ uma medida de probabilidade que possui a

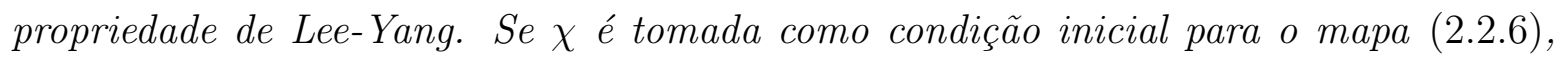
então $\sigma_{k}^{(N)}(y)$, para todo $k=1,2,3, \ldots$, também possui a propriedade de Lee-Yang.

Isso significa que para todo $k=1,2,3, \ldots$ temos

$$
\phi_{k}^{(N)}(z)=\int_{\mathbb{R}^{N}} \exp \{i z \cdot y\} d \sigma_{k}^{(N)}(y)=\varphi_{k}^{(N)}(x), \quad x=-|z|^{2},
$$

$\operatorname{com} \varphi_{k}^{(N)} \in\{f \in \mathcal{L}: f(0)=1\}$. Considerando a parte radial (nessa expressão $r=|z|$ )

$$
\frac{\partial^{2}}{\partial r^{2}}+\frac{N-1}{r} \frac{\partial}{\partial r}=-4\left(x \frac{\partial^{2}}{\partial x^{2}}+\frac{N}{2} \frac{\partial}{\partial x}\right) \equiv-4 \Delta_{N / 2}
$$


do operador laplaceano $N$-dimensional $\Delta=\partial^{2} / \partial z_{1}^{2}+\cdots+\partial^{2} / \partial z_{N}^{2}$, reescrevemos $(2.2 .16)$ como

$$
\varphi_{k}^{(N)}(x)=\frac{1}{M_{k}} \exp \left\{2 c_{\gamma, k}(L-1) \Delta_{N / 2}\right\}\left(\varphi_{k-1}^{(N)}\left(L^{-\gamma} x\right)\right)^{L^{d}}
$$

sendo $M_{k}$ uma normalização tal que $\varphi_{k}^{(N)}(0)=1$ (trata-se de (2.2.22) reescrita em termos de $\Delta_{N / 2}$ e $\left.\varphi_{k-1}^{(N)}(x)\right)$; o que acabamos de aprender com o Teorema 2.2 .12 é que o mapa (2.2.40) preserva a classe $\{f \in \mathcal{L}: f(0)=1\}$. Por conseguinte, valem as desigualdades estabelecidas por Newman $([44])$ : sendo um elemento de $\{f \in \mathcal{L}: f(0)=1\}, \varphi_{k}^{(N)}(x)$ é descrita pelos parâmetros $\lambda_{k}^{(N)}$ e $\left\{-\gamma_{n, k}^{(N)}, n \geq 1\right\}$ (vide representação (2.2.26)); logo

$$
\begin{aligned}
\ln \varphi_{k}^{(N)}(x) & =\lambda_{k}^{(N)} x+\sum_{n=1}^{\infty} \ln \left(1+\frac{x}{\gamma_{n, k}^{(N)}}\right) \\
& =\lambda_{k}^{(N)} x+\sum_{j=1}^{\infty}\left(\frac{(-1)^{j+1}}{j} \sum_{n=1}^{\infty}\left(\gamma_{n, k}^{(N)}\right)^{-j}\right) x^{j} \\
& =\sum_{j=1}^{\infty} \nu_{j, k}^{(N)} x^{j}
\end{aligned}
$$

com

$$
\nu_{1, k}^{(N)}=\lambda_{k}^{(N)}+\sum_{n=1}^{\infty}\left(\gamma_{n, k}^{(N)}\right)^{-1} \quad \text { e } \quad \nu_{j, k}^{(N)}=\frac{(-1)^{j+1}}{j} \sum_{n=1}^{\infty}\left(\gamma_{n, k}^{(N)}\right)^{-j} \quad(j \geq 2),
$$

sendo que a expansão usada na segunda igualdade é válida desde que $|x|<\gamma_{1, k}^{(N)}$. O Teorema 6 de [44] diz que

$$
0 \leq(-1)^{j+1} j \nu_{j, k}^{(N)} \leq\left(-2 \nu_{2, k}^{(N)}\right)^{j / 2}, \quad j \geq 3
$$

Se ao longo de uma trajetória $\left\{\varphi_{k}^{(N)}(x)\right\}_{k=1}^{\infty}$ o coeficiente $\nu_{2, k}^{(N)}$ convergir para zero, então todos coeficientes de ordem superior $\nu_{j, k}^{(N)}, j \geq 3$, convergem igualmente para zero. Nesse caso teríamos, por (2.2.41) e (2.2.38), a convergência 


$$
\phi_{k}^{(N)}(z)=\exp \left(\sum_{j=1}^{\infty} \nu_{j, k}^{(N)}(-|z|)^{2 j}\right) \longrightarrow e^{-\nu_{1, \infty}^{(N)}|z|^{2}}
$$

Comentário 2.2.13 Os coeficientes $\nu_{j, k}^{(N)}$ relacionam-se com os cumulantes de ordem par $\kappa_{2 j, k}^{(N)}$ da distribuição $\sigma_{k}^{(N)}(y)$ pela relação

$$
\nu_{j, k}^{(N)}=\frac{\kappa_{2 j, k}^{(N)}}{(2 j) !}, \quad j \geq 1
$$

lembrando que

$$
\kappa_{j, k}^{(N)}:=\left.(-i)^{j} \frac{d^{j} \ln \phi_{k}^{(N)}(z)}{d z^{j}}\right|_{z=0}
$$

Os cumulantes de ordem impar $\kappa_{2 j-1, k}^{(N)}$ são nulos por conta da simetria apresentada por $\phi_{k}^{(N)}(z)$.

Antes de adentrarmos ao artigo [32], precisamos realizar algumas modificações: pela mudança de variável $\boldsymbol{y} \rightarrow \sqrt{\beta} \boldsymbol{y}$ em (2.1.1) (lembrar da Observação 2.2.2), (2.2.16) passa a ser escrita

$$
\phi_{k}^{(N)}(z)=\frac{1}{N_{k}} \exp \left\{-\frac{\beta c_{\gamma, k}(L-1)}{2} \Delta\right\}\left(\phi_{k-1}^{(N)}\left(L^{-\gamma / 2} z\right)\right)^{L^{d}}
$$

e conseqüentemente

$$
\varphi_{k}^{(N)}(x)=\frac{1}{M_{k}} \exp \left\{2 \beta c_{\gamma, k}(L-1) \Delta_{N / 2}\right\}\left(\varphi_{k-1}^{(N)}\left(L^{-\gamma} x\right)\right)^{L^{d}}
$$

Por fim, desconsiderando a normalização $M_{k}$ e escolhendo $\gamma=d+2$ (e portanto $\left.c_{\gamma, k}=1\right)$, obtemos a relação recursiva

$$
f_{k}(x)=\exp \left\{2 \beta(L-1) \Delta_{N / 2}\right\}\left(f_{k-1}\left(L^{-(d+2)} x\right)\right)^{L^{d}}
$$

A atenção de [32] é justamente para esse mapa. 
Observação 2.2.14 Pela Proposição 2.2.11 e sua demonstração,

$$
f^{\text {gauss }}(x)=L^{-N /\left(L^{d}-1\right)} e^{\frac{L+1}{2 \beta} x}
$$

é um ponto fixo de (2.2.47).

Seja $\mathcal{E}$ o conjunto de todas as funções inteiras de $\mathbb{C}$ em $\mathbb{C}$. Para $b>0$ definamos

$$
\|f\|_{b}:=\sup _{j \in \mathbb{N}} \frac{1}{b^{j}}\left|\frac{d^{j} f(0)}{d x^{j}}\right| .
$$

Para $a \geq 0$ seja

$$
\mathcal{A}_{a}=\left\{f \in \mathcal{E}:(\forall b>a)\|f\|_{b}<\infty\right\}
$$

Por fim, sejam

$$
\begin{gathered}
\mathcal{L}^{+}=\{f \in \mathcal{L}: f(0)>0\}, \quad \mathcal{L}^{(1)}=\{f \in \mathcal{L}: f(0)=1\} \\
\mathcal{L}_{a}=\mathcal{L} \cap \mathcal{A}_{a}, \quad \mathcal{L}_{a}^{+}=\mathcal{L}^{+} \cap \mathcal{A}_{a}, \quad \mathcal{L}_{a}^{(1)}=\mathcal{L}^{(1)} \cap \mathcal{A}_{a} .
\end{gathered}
$$

Consideremos o seguinte problema de Cauchy

$$
\begin{aligned}
\frac{\partial f(t, x)}{\partial t} & =\left(\Delta_{N / 2} f\right)(t, x), \quad t \in \mathbb{R}_{+}, \quad x \in \mathbb{C}, \\
f(0, x) & =g(x),
\end{aligned}
$$

e que a condição inicial tenha a forma

$$
g(x)=\exp (-\varepsilon x) h(x), \quad h \in \mathcal{A}_{0}, \quad \varepsilon \geq 0 .
$$

O seguinte resultado foi demonstrado em [31] pelo Teorema 1.6. 
Proposição 2.2.15 (i) Para todo $N \geq 0$ e $g \in \mathcal{E}$ tendo a forma (2.2.54), o problema (2.2.53) tem uma única solução em $\mathcal{A}_{\varepsilon}$, a qual possui a seguinte representação integral

$$
f(t, x)=\exp \left(-\frac{x}{t}\right) \int_{0}^{+\infty} s^{N / 2-1} w_{N / 2}\left(\frac{x s}{t}\right) e^{-s} g(t s) d s
$$

sendo $t>0 e$

$$
w_{N / 2}(x)=\sum_{k=0}^{\infty} \frac{x^{k}}{k ! \Gamma(N / 2+k)} .
$$

(ii) Se em (2.2.54) $\varepsilon>0$, a solução (2.2.55) converge para zero em $\mathcal{A}_{\varepsilon}$ quando $t \rightarrow \infty$.

(iii) Se em (2.2.54) $h \in \mathcal{L}_{0}$ e $\varepsilon=0$, a solução (2.2.55) também pertence a $\mathcal{L}_{0}$. Ela diverge quando $t \rightarrow \infty$, o que significa que $M_{f}(t, r) \rightarrow \infty$ para todo $r \in \mathbb{R}_{+}$. Aqui

$$
M_{f}(t, r)=\sup _{|x| \leq r}|f(t, x)|
$$

Consideremos agora a seguinte modificação do problema (2.2.53): dividamos o semieixo do tempo $\mathbb{R}_{+}$em intervalos $\mathcal{I}_{k}=[2 \beta(L-1)(k-1), 2 \beta(L-1) k], k=1,2,3, \ldots$, com

$$
\mathbb{R}_{+}=\bigcup_{k=1}^{\infty} \mathcal{I}_{k}
$$

Em cada intervalo $\mathcal{I}_{k}$ a evolução é descrita por (2.2.53), mas nos tempos $t=2 \beta(L-1) k$ $(k=1,2,3, \ldots)$ a função é modificada da seguinte maneira

$$
f(2 \beta(L-1) k, x) \rightarrow\left(f\left(2 \beta(L-1) k, L^{-(d+2)} x\right)\right)^{L^{d}}
$$

com $d<\infty$ fixo e $L^{d} \geq 2$ um inteiro. É mais conveniente trabalhar com a seqüência de funções dependentes de $t$ pertencente a um certo intervalo a considerar uma função com 
$t$ variando sobre uma seqüência de intervalos. No que segue, considera-se a seqüência de funções $\left\{f_{k}(t, x)\right\}_{k=1}^{\infty}$, cada uma delas sendo a solução do seguinte problema de Cauchy

$$
\begin{aligned}
\frac{\partial f_{k}(t, x)}{\partial t} & =2 \beta(L-1)\left(\Delta_{N / 2} f_{k}\right)(t, x), \quad t \in[0,1], \quad x \in \mathbb{C}, \\
f_{k}(0, x) & =\left(f_{k-1}\left(1, L^{-(d+2)} x\right)\right)^{L^{d}}, \quad k=1,2,3, \ldots \\
f_{0}(1, x) & =g(x), \quad g \in \mathcal{L}^{+} .
\end{aligned}
$$

Qualquer $g \in \mathcal{L}^{+}$é descrita pelos parâmetros $C, \lambda$ e $\left\{-\gamma_{n}, n \geq 1\right\}$ (vide (2.2.51) e a representação $(2.2 .26)$ ), e pode-se mostrar que tal $g$ pertence a $\mathcal{L}_{\lambda}^{+}$. Dado $g \in \mathcal{L}^{+}$, definimos

$$
m_{j}(g)=\sum_{n=1}^{\infty} \gamma_{n}^{-j}, \quad j=1,2,3, \ldots
$$

e

$$
I(g)=\left\{\begin{array}{ll}
{[0,(L+1) / 2 \lambda]} & \text { se } \lambda>0 \\
{[0, \infty)} & \text { se } \lambda=0
\end{array} .\right.
$$

A Proposição 2.2.15 implica a existência de soluções de (2.2.60) ao menos para $g \in \mathcal{L}_{0}$. O teorema a seguir estabelece a existência de soluções desse problema em uma situação mais geral.

Teorema 2.2.16 Sejam $g \in \mathcal{L}^{+}$e $\beta \in I(g)$ dados. Então para todo $k=1,2,3, \ldots$ e $N \geq 0$, o problema (2.2.60) tem uma única solução $f_{k}$, a qual pertence a $\mathcal{L}_{\lambda}^{+}$.

Para $\beta=0$ a seqüência $\left\{f_{k}(t, x)\right\}_{k=1}^{\infty}$ pode ser encontrada explicitamente:

$$
f_{k}(t, x)=\left(g\left(L^{-(d+2) k} x\right)\right)^{L^{d k}}
$$


Se $g \in \mathcal{L}^{(1)}$, tal seqüência converge em $\mathcal{A}_{\lambda}$ para a função $f(t, x) \equiv 1$. Podemos assim esperar que a mesma convergência, ou similar, seja válida também para valores pequenos de $\beta$. Por outro lado, para valores grandes de $\beta$, a afirmação (iii) da Proposição 2.2.15 sugere que há divergência. O objetivo dos autores nesse trabalho foi estudar as seguintes questões: (a) existe um valor intermediário de $\beta$, digamos $\beta_{c}$, que separa valores "grandes" e "pequenos" desse parâmetro?; (b) qual seria a convergência da seqüência $\left\{f_{k}(t, x)\right\}_{k=1}^{\infty}$ para $\beta=\beta_{c}$ ? A resposta foi encontrada para $4<d<\infty$ e $g$ escolhida em um subconjunto de $\mathcal{L}^{+}$definido por $d$ como segue:

Definição 2.2.17 A família $\mathcal{L}(d)$ consiste das funções $g \in \mathcal{L}^{(1)}$ que não são constantes e são tais que

$$
\frac{m_{2}(g)}{\left(\lambda+m_{1}(g)\right)^{2}} \leq \frac{L^{d / 2}}{N / 2+1} \frac{L^{\frac{d-4}{4}}-1}{L^{\frac{d+4}{4}}-1}, \quad \frac{m_{2}(g)}{\left(m_{1}(g)\right)^{2}} \leq \frac{L^{d / 2}}{N / 2+1}
$$

Os autores então enunciam seu principal teorema:

Teorema 2.2.18 Para todo $N \geq 0,4<d<\infty$ e $g \in \mathcal{L}(d)$, com $L^{d} \geq 2$ um inteiro, existe $\beta_{c} \in I(g)$ positivo e uma função $C:\left[0, \beta_{c}\right] \rightarrow \mathbb{R}_{+}$tal que

(i) para $\beta<\beta_{c}$, a seqüência de soluções de (2.2.60)

$$
\left\{f_{k}(t, x): k=1,2,3, \ldots, f_{0}(1, x)=C(\beta) g(x)\right\}
$$

converge em $\mathcal{A}_{\kappa^{-1}}$, com $\kappa=2 \beta_{c} /(L+1)$, para a função $f(t, x) \equiv 1$;

(ii) para $\beta=\beta_{c}$, a seqüência

$$
\left\{f_{k}(t, x): k=1,2,3, \ldots, f_{0}(1, x)=C\left(\beta_{c}\right) g(x)\right\}
$$

converge em $\mathcal{A}_{\kappa^{-1}}$ para

$$
f^{*}(t, x)=\frac{L^{-N /\left(L^{d}-1\right)}}{\left(L^{2}-t\left(L^{2}-1\right)\right)^{N / 2}} \exp \left(\frac{L+1}{2 \beta_{c}} \frac{x}{L^{2}-t\left(L^{2}-1\right)}\right) .
$$


Comentário 2.2.19 O modelo $O(N)$ de Heisenberg hierárquico exibe transição de fase para $d>2$ (vide [5]): lembrando que $\left|\Lambda_{K}\right|=L^{K d}=n$, que $\Omega_{n}=\mathbb{R}^{N . n}$ denota o espaço de configuração e $d \nu_{n}^{(N)}(\boldsymbol{y})$ a medida de Gibbs do modelo, seja

$$
m^{2}:=\lim _{n \rightarrow \infty} \int_{\Omega_{n}}\left(\frac{1}{n} \sum_{\boldsymbol{i} \in \Lambda_{K}} y_{\boldsymbol{i}}\right)^{2} d \nu_{n}^{(N)}(\boldsymbol{y})
$$

para $d>2$ existe $\beta_{c}=\beta_{c}(d, L, N)$ tal que $m^{2}>0$ se $\beta>\beta_{c}$ e $m^{2}=0$ se $\beta<\beta_{c}$. O valor $\beta_{c}$ recebe o nome de temperatura inversa crítica. Quando $\beta=\beta_{c}$ diz-se que o sistema está na criticalidade.

O Teorema 2.2.18 responde, para todo $N \geq 0,4<d<\infty, g \in \mathcal{L}(d)$ e $L^{d} \geq 2$ um inteiro, a questão da convergência da seqüência de funções características $\left\{\phi_{k}^{(N)}(z)\right\}_{k=1}^{\infty}$ gerada por (2.2.16) no caso em que a variável soma (2.2.3) é normalizada com a normalização anormal $(\gamma=d+2)$. A convergência mostrada na afirmação $(i i)$, que implica a convergência $\phi_{k}^{(N)}(z) \rightarrow \phi^{\text {gauss }}(z)=e^{-\frac{L+1}{2}|z|^{2}}$ (ou seja, convergência para o ponto fixo gaussiano), nos diz que tal normalização é adequada para tratar o sistema na criticalidade, situação em que as variáveis de spin estão fortemente correlacionadas. Por outro lado, a afirmação $(i)$ nos diz que essa normalização é muito forte para tratar o caso $\beta<\beta_{c}$; para esse regime de temperaturas vale o Teorema Central do Limite usual, isto é, devemos escolher a normalização normal $(\gamma=d)$ na variável soma (2.2.3) para haver convergência para uma função gaussiana $e^{-C|z|^{2}}$, com $C>0$ uma constante que possivelmente depende de $d, L, N$ e $\beta$ - vide Teorema 2 de [30] ou Teorema 1.6 de [32].

Assim como Kozitsky, em [49] Watanabe também estabelece o Teorema Central do Limite para o modelo $O(N)$ de Heisenberg hierárquico para o caso de normalização anormal $(\gamma=d+2)$. Todavia, seu resultado é válido para $N$ suficientemente grande, $4 \leq d<\infty$ (note a inclusão de $d=4), L^{d}=2$ e utilizando como condição inicial a função característica $\phi_{0}^{(N)}(z)$ dada por (2.2.17), que é a condição inicial definida pelo próprio modelo. Para controlar a trajetória $O(N)$, que parte de muito longe do ponto fixo gaussiano, Watanabe utiliza a trajetória exatamente solúvel $O(\infty)$ juntamente com dois ingredientes válidos para $L^{d}$ inteiro: o fato de o mapa preservar a propriedade de Lee-Yang, e a propriedade de positividade por reflexão. O primeiro ingrediente nos trás às mãos a desigualdade (2.2.43), a qual é uma ferramenta para controle da convergência da seqüência $\left\{\nu_{j, k}^{(N)}\right\}_{k=1}^{\infty}$ (vide (2.2.41)-(2.2.42)). O segundo ingrediente habilita-nos a 
evocar um resultado sobre a expansão $1 / N$ desenvolvida por Kupiainen na referência [35], ferramenta que acaba por garantir a convergência uniforme da trajetória $O(N)$ para a trajetória $O(\infty)$.

Comentário 2.2.20 Consideremos a expansão (2.2.41) escalada em $N$

$$
-\frac{1}{N} \ln \varphi_{k}^{(N)}(N x)=\sum_{j=1}^{\infty} \mu_{j, k}^{(N)} x^{j}
$$

$\mu_{j, k}^{(N)}:=-N^{j-1} \nu_{j, k}^{(N)} \operatorname{com} \nu_{j, k}^{(N)}$ definido em (2.2.42). O que Watanabe [49] mostra é que para cada $j \geq 1$ e para cada $k \geq 0$

$$
\lim _{N \rightarrow \infty} \mu_{j, k}^{(N)}=\mu_{j, k}^{(\infty)}
$$

sendo a convergência uniforme em $\beta$ sobre qualquer subconjunto compacto de $(0, \infty)$ vide Proposição 2.3 de [49]. Esse resultado é provado pelo uso da expansão $1 / N$ exposta por Kupiainen no artigo [35].

O método de Kupiainen para sistemas de spins sobre redes regulares requer que o funcional definido pela medida de Gibbs do modelo satisfaça a propriedade de positividade por reflexão - vide [20] para uma exposição da teoria abstrata. Então Watanabe mostra, no apêndice de seu artigo, que o funcional definido pela medida de Gibbs do modelo $O(N)$ de Heisenberg hierárquico satisfaz a citada propriedade (para $L^{d}=2$ - em [8] estendemos sua prova para o caso geral $L>1$ e $d \geq 1$ ), e com isso garante que o método de Kupiainen pode ser aplicado para o modelo hierárquico em questão.

\subsection{A Aproximação de Potencial Local $(L \downarrow 1)$}

Embora a transformação (2.2.16) seja discreta, nos trabalhos de Kozitsky e Watanabe parte da dinâmica é evoluída através da equação do calor. O presente trabalho diferencia-se desses dois pela utilização da chamada aproximação de potencial local: definindo a função 


$$
U(t, z)=-\ln \phi_{k}^{(N)}(z)
$$

com parâmetro de escala

$$
t=k \ln L
$$

tomaremos conjuntamente os limites $k \rightarrow \infty$ e $L \downarrow 1$ de tal maneira que $k \ln L$ permaneça fixo em um número real positivo $t$. Com esse procedimento, como veremos na próxima proposição, a órbita discreta $\left\{-\ln \phi_{k}^{(N)}(z)\right\}_{k=0}^{\infty}$ torna-se contínua, e a dinâmica completa se reduz a uma equação a derivadas parciais. O limite da dimensão do bloco $L$ para 1 que é a chamada aproximação de potencial local [17].

Proposição 2.3.1 A função $U(t, z)$ definida por (2.3.1) e (2.3.2) satisfaz, no limite conjunto $k \rightarrow \infty$ e $L \downarrow 1$, a equação

$$
U_{t}=-\frac{c_{\gamma}(t)}{2}\left(\Delta U-\left|U_{z}\right|^{2}\right)-\frac{\gamma}{2} z \cdot U_{z}+d U+\left.\frac{c_{\gamma}(t)}{2}\left(\Delta U-\left|U_{z}\right|^{2}\right)\right|_{z=0},
$$

$t \in \mathbb{R}_{+} e z \in \mathbb{R}^{N}$, com condição inicial

$$
U(0, z)=-\ln \left[\frac{\Gamma(N / 2)}{(\sqrt{\beta N}|z| / 2)^{N / 2-1}} J_{N / 2-1}(\sqrt{\beta N}|z|)\right]
$$

O último termo em (2.3.3) é um multiplicador de Lagrange que garante que $U(t, 0)=0$ para todo $t \geq 0$, haja vista que $\phi_{k}^{(N)}(0)=1$ para todo $k=0,1,2, \ldots$ e todo $L$ e a definição (2.3.1) da função $U(t, z) ; U_{z}=\partial U / \partial z \operatorname{com} \partial / \partial z=\left(\partial / \partial z_{1}, \ldots, \partial / \partial z_{N}\right) ; e$

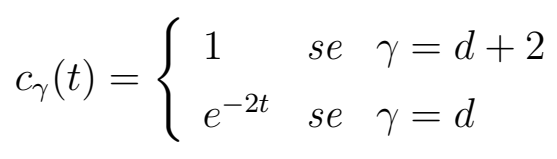

é $c_{\gamma, k}(2.2 .5)$ escrito em termos de $t$, considerando-se (2.3.2). 
Demonstração. Da definição de derivada, pela definição (2.3.1) da função $U(t, z)$, pela relação (2.2.16), e da observação que $t-\ln L=(k-1) \ln L$, temos

$$
\begin{aligned}
U_{t} & =\lim _{L \downarrow 1} \frac{U(t, z)-U(t-\ln L, z)}{\ln L} \\
& =\lim _{L \downarrow 1} \frac{1}{\ln L}\left\{-\ln \left[\frac{1}{N_{k}} \exp \left\{-\frac{c_{\gamma, k}(L-1)}{2} \Delta\right\}\left(\phi_{k-1}^{(N)}\left(L^{-\gamma / 2} z\right)\right)^{L^{d}}\right]+\ln \phi_{k-1}^{(N)}(z)\right\} \\
& =\lim _{k \rightarrow \infty} \frac{k}{t}\left\{-\ln \left[\frac{1}{N_{k}} \exp \left\{-\frac{c_{\gamma}(t)\left(e^{t / k}-1\right)}{2} \Delta\right\}\left(\phi_{k-1}^{(N)}\left(e^{-\gamma t / 2 k} z\right)\right)^{e^{d t / k}}\right]+\ln \phi_{k-1}^{(N)}(z)\right\},
\end{aligned}
$$

com $N_{k}$ dado por (2.2.22).

Notemos agora que para $k$ grande valem as seguintes expansões:

$$
\exp \left\{-\frac{c_{\gamma}(t)\left(e^{t / k}-1\right)}{2} \Delta\right\}=1-\frac{t}{k} \frac{c_{\gamma}(t)}{2} \Delta+O\left((t / k)^{2}\right)
$$

$\mathrm{e}$

$$
\begin{gathered}
\left(\phi_{k-1}^{(N)}\left(e^{-\gamma t / 2 k} z\right)\right)^{e^{d t / k}}= \\
=\phi_{k-1}^{(N)}(z)+\phi_{k-1}^{(N)}(z)\left(-\frac{\gamma}{2} z \cdot \frac{\partial \ln \phi_{k-1}^{(N)}(z)}{\partial z}+d \ln \phi_{k-1}^{(N)}(z)\right) \frac{t}{k}+O\left((t / k)^{2}\right) .
\end{gathered}
$$

Substituindo tais expressões na última igualdade de (2.3.6), e utilizando a expansão

$$
\ln \left(a_{0}+a_{1} \delta+O\left(\delta^{2}\right)\right)=\ln a_{0}+\frac{a_{1}}{a_{0}} \delta+O\left(\delta^{2}\right)
$$

para $\delta$ pequeno e $a_{0}>0$, obtemos

$$
U_{t}=\lim _{k \rightarrow \infty}\left\{\frac{c_{\gamma}(t)}{2} \frac{\Delta \phi_{k-1}^{(N)}(z)}{\phi_{k-1}^{(N)}(z)}+\frac{\gamma}{2} z \cdot \frac{\partial \ln \phi_{k-1}^{(N)}(z)}{\partial z}-d \ln \phi_{k-1}^{(N)}(z)-\frac{c_{\gamma}(t)}{2} \Delta \phi_{k-1}^{(N)}(0)+O(t / k)\right\} .
$$


Note que o último termo é justamente (lembrar que $\phi_{k}^{(N)}(0)=1$ para todo $\left.k=0,1,2, \ldots\right)$

$$
-\frac{c_{\gamma}(t)}{2} \frac{\Delta \phi_{k-1}^{(N)}(z)}{\phi_{k-1}^{(N)}(z)}-\frac{\gamma}{2} z \cdot \frac{\partial \ln \phi_{k-1}^{(N)}(z)}{\partial z}+\left.d \ln \phi_{k-1}^{(N)}(z)\right|_{z=0},
$$

proveniente da expansão de $\ln N_{k}$.

Por fim, pelas igualdades

$$
\begin{gathered}
\frac{\Delta \phi_{k-1}^{(N)}(z)}{\phi_{k-1}^{(N)}(z)}=\Delta \ln \phi_{k-1}^{(N)}(z)+\left|\frac{\partial \ln \phi_{k-1}^{(N)}(z)}{\partial z}\right|^{2} \\
\Delta \phi_{k-1}^{(N)}(0)=\Delta \ln \phi_{k-1}^{(N)}(0)+\left|\frac{\partial \ln \phi_{k-1}^{(N)}(0)}{\partial z}\right|^{2}
\end{gathered}
$$

$\mathrm{e} \ln \phi_{k-1}^{(N)}(z)=-U(t-t / k, z)$, obtemos $(2.3 .3)$

Quanto à condição inicial (2.3.4), esta segue imediatamente do fato que

$$
U(0, z)=-\ln \phi_{0}^{(N)}(z)
$$

e de (2.2.17).

Observação 2.3.2 Para $\gamma=d+2$, segue por (2.2.34) e (2.3.1) que

$$
U^{\text {gauss }}(z)=\lim _{L \downarrow 1}\left\{-\ln \phi^{\text {gauss }}(z)\right\}=|z|^{2}
$$

é um ponto fixo da equação (2.3.3).

Dado que a condição inicial (2.3.4) depende de $|z|$ e a equação de evolução (2.3.3) preserva a simetria esférica, segue que $U(t, z)=U(t,|z|)$ para todo $t \geq 0$. É assim conveniente e suficiente que trabalhemos com a variável $x=-|z|^{2}$. Definindo a função adequadamente escalada em $N$ 


$$
u^{(N)}(t, x)=\frac{1}{N} U(t, \sqrt{N} z)
$$

enunciamos:

Proposição 2.3.3 Em termos da função $u^{(N)}(t, x)$ definida por (2.3.16), o problema de valor inicial (2.3.3)-(2.3.4) fica

$$
u_{t}^{(N)}=c_{\gamma}(t)\left(\frac{2}{N} x u_{x x}^{(N)}+u_{x}^{(N)}-2 x\left(u_{x}^{(N)}\right)^{2}\right)-\gamma x u_{x}^{(N)}+d u^{(N)}-c_{\gamma}(t) u_{x}^{(N)}(t, 0)
$$

com

$$
u^{(N)}(0, x)=-\frac{1}{N} \ln \left[\frac{\Gamma(N / 2)}{(i \sqrt{\beta x} N / 2)^{N / 2-1}} J_{N / 2-1}(i \sqrt{\beta x} N)\right] .
$$

$O$ último termo em (2.3.17) garante que $u^{(N)}(t, 0)=0$ para todo $t \geq 0$.

Demonstração. Seja $r=|z|$ (e portanto $\left.x=-r^{2}\right)$ e

$$
V(t, x)=U(t, z)
$$

Lembrando que a parte radial do operador laplaceano $N$-dimensional $\Delta$ é dada por (2.2.39), temos que

$$
\Delta U(t, z)=U_{r r}(t, r)+\frac{N-1}{r} U_{r}(t, r)=-4 x V_{x x}(t, x)-2 N V_{x}(t, x) .
$$

Além disso,

$$
\left|U_{z}(t, z)\right|^{2}=\left(U_{r}(t, r)\right)^{2}=-4 x\left(V_{x}(t, x)\right)^{2}
$$

$\mathrm{e}$ 


$$
z \cdot U_{z}(t, z)=r U_{r}(t, r)=2 x U_{x}(t, x) .
$$

Portanto $V(t, x)$ satisfaz a equação

$$
V_{t}=c_{\gamma}(t)\left(2 x V_{x x}+N V_{x}-2 x\left(V_{x}\right)^{2}\right)-\gamma x V_{x}+d V-c_{\gamma}(t) N V_{x}(t, 0)
$$

com condição inicial

$$
V(0, x)=-\ln \left[\frac{\Gamma(N / 2)}{(i \sqrt{\beta N x} / 2)^{N / 2-1}} J_{N / 2-1}(i \sqrt{\beta N x})\right] .
$$

Agora, pelas definições (2.3.16) e (2.3.19) temos que

$$
u^{(N)}(t, x)=\frac{1}{N} V(t, N x)
$$

Considerando então a mudança de escala $x \rightarrow N x$ e o fator multiplicativo $1 / N$ em (2.3.23)-(2.3.24), chega-se a (2.3.17)-(2.3.18).

Observação 2.3.4 Para $\gamma=d+2$, por (2.3.15) e (2.3.16) temos que

$$
u^{\text {gauss }}(x)=\frac{1}{N} U^{\text {gauss }}(\sqrt{N} z)=-x
$$

é um ponto fixo da equação (2.3.17).

O problema de valor inicial (2.3.17)-(2.3.18), com $t \in \mathbb{R}_{+}$e $x \in \mathbb{C}$, será o ponto de partida de nossos estudos; como nosso interesse é o sistema na criticalidade, daqui por diante mantem-se a escolha $\gamma=d+2$ fixa. 


\section{Capítulo 3}

\section{Definições e Ferramentas}

\subsection{Teoria de Somabilidade: Definições}

Série Formal de Potências. Seja $\mathcal{O}(r)$ o anel das funções analíticas sobre o disco fechado $\overline{D_{r}\left(z_{0}\right)}$, o fecho de $D_{r}\left(z_{0}\right):=\left\{z \in \mathbb{C}:\left|z-z_{0}\right|<r\right\}$. Seja $\mathcal{O}(r)[[\varepsilon]]$ o anel das séries formais em $\varepsilon$ sobre o anel $\mathcal{O}(r)$; define-se $\mathcal{O}[[\varepsilon]]$ por

$$
\mathcal{O}[[\varepsilon]]:=\bigcup_{r>0} \mathcal{O}(r)[[\varepsilon]]
$$

Um elemento $\hat{f}(\varepsilon, z) \in \mathcal{O}[[\varepsilon]]$ é escrito

$$
\hat{f}(\varepsilon, z)=\sum_{i=0}^{\infty} f_{i}(z) \varepsilon^{i}
$$

onde $f_{i}(z) \in \mathcal{O}(r)$ para algum $r>0$.

Série Formal $s$-Gevrey. Seja $s$ um número não negativo. $\mathcal{O}(r)[[\varepsilon]]_{s}$, que é chamada de Classe Gevrey $s$ ou simplesmente $s$-Gevrey, é o subanel de $\mathcal{O}(r)[[\varepsilon]]$ cujos coeficientes satisfazem, para constantes positivas $C$ e $\mu$, a desigualdade

$$
\max _{z \in \overline{D_{r}\left(z_{0}\right)}}\left|f_{i}(z)\right| \leq C i !^{s} \mu^{i} \quad \text { para } i=0,1,2, \ldots
$$


Também se define $\mathcal{O}[[\varepsilon]]_{s}$, o conjunto de todas as séries em $\varepsilon$ que são $s$-Gevrey, por

$$
\mathcal{O}[[\varepsilon]]_{s}:=\bigcup_{r>0} \mathcal{O}(r)[[\varepsilon]]_{s}
$$

Em particular, $\mathcal{O}[[\varepsilon]]_{0}$ é o conjunto de todas as séries em $\varepsilon$ que são convergentes.

Expansão Assintótica de Gevrey. Seja $\theta \in \mathbb{R}, \alpha>0$ e $0<E \leq \infty$. Denotamos por $S(\theta, \alpha ; E)$ o domínio setorial de abertura $\alpha$ e raio $E$ cuja bissetriz é o eixo $\theta$ :

$$
S(\theta, \alpha ; E):=\{\varepsilon \in \mathbb{C}:|\arg \varepsilon-\theta|<\alpha / 2,0<|\varepsilon|<E\}
$$

Se o raio $E$ não é tão importante, simplesmente denotaremos tal domínio setorial por $S(\theta, \alpha)$. Um domínio setorial $S^{\prime}$ é dito ser um subsetor próprio de $S(\theta, \alpha ; E)$ se seu fecho está contido em $S(\theta, \alpha ; E) \cup\{0\}$.

Seja $f(\varepsilon, z)$ uma função analítica em $\bigcap_{\alpha^{\prime}<\alpha} S\left(\theta, \alpha^{\prime}\right) \times D_{r\left(\alpha^{\prime}\right)}\left(z_{0}\right)$, onde $r\left(\alpha^{\prime}\right)$ pode tender a 0 quando $\alpha^{\prime} \rightarrow \alpha$. Definimos:

Definição 3.1.1 $\hat{f}(\varepsilon, z) \in \mathcal{O}[[\varepsilon]]_{s}$ é dita ser uma expansão assintótica de Gevrey de $f(\varepsilon, z)$ quando $\varepsilon \rightarrow 0$ em $S(\theta, \alpha)$ se para qualquer subsetor próprio $S^{\prime} \subset S(\theta, \alpha ; E)$ (com raio suficientemente pequeno), existem constantes positivas $C, \mu$ e $0<r_{1}<r$ tais que

(i) $\hat{f}(\varepsilon, z) \in \mathcal{O}\left(r_{1}\right)[[\varepsilon]]_{s}$,

$$
\max _{z \in \overline{D_{r_{1}}\left(z_{0}\right)}}\left|f(\varepsilon, z)-\sum_{i=0}^{I-1} f_{i}(z) \varepsilon^{i}\right| \leq C I !^{s} \mu^{I}|\varepsilon|^{I}, \quad \varepsilon \in S^{\prime}, \quad I=1,2,3, \ldots
$$

Definição 3.1.2 Uma função analítica $f(\varepsilon, z)$ é dita ser Gevrey ordem $s$ assintoticamente expansiva em $S(\theta, \alpha)$ se possui uma expansão assintótica de Gevrey $\hat{f}(\varepsilon, z) \in$ $\mathcal{O}[[\varepsilon]]_{s}$. 
Denotemos por $\mathcal{A}^{(s)}(S(\theta, \alpha))$ o conjunto das funções analíticas que são Gevrey ordem $s$ assintoticamente expansivas em $S(\theta, \alpha)$. Definimos o mapa $J^{(s)}$ por

$$
J^{(s)}: \mathcal{A}^{(s)}(S(\theta, \alpha)) \longrightarrow \mathcal{O}[[\varepsilon]]_{s}
$$

onde $J^{(s)}(f(\varepsilon, z))=\hat{f}(\varepsilon, z)$ é a expansão assintótica de Gevrey $\hat{f}(\varepsilon, z)$ de $f(\varepsilon, z)$.

Teorema 3.1.3 (1) [setor de abertura estreita] O mapa $J^{(s)}$ definido por (3.1.7) é sobrejetivo, mas não injetivo, para qualquer $\theta \in \mathbb{R}$ e $\alpha$ com $\alpha \leq s \pi$.

(2) [setor de abertura ampla] Para qualquer $\alpha \operatorname{com} \alpha>s \pi$, o mapa $J^{(s)}$ não é sobrejetivo, mas é injetivo, para qualquer $\theta \in \mathbb{R}$.

Veja [1] para uma prova deste teorema quando a função $f=f(\varepsilon)$ depende apenas de $\varepsilon$.

\section{$s$-Somabilidade em uma Direção $\theta$.}

Definição 3.1.4 Sejam $s>0, \theta \in \mathbb{R}$ e $\hat{f}(\varepsilon, z) \in \mathcal{O}[[\varepsilon]]$ dados. Diz-se que $\hat{f}(\varepsilon, z) e ́$ $s$-somável na direção $\theta$ se um setor $S(\theta, \alpha ; E)$, com $\alpha>s \pi$, e uma função $f(\varepsilon, z) \in$ $\mathcal{A}^{(s)}(S(\theta, \alpha ; E))$ existem com $J^{(s)}(f(\varepsilon, z))=\hat{f}(\varepsilon, z)$. Tal $f(\varepsilon, z)$ é dita ser a $s$-soma de $\hat{f}(\varepsilon, z)$ na direção $\theta$.

Transformada Formal de Borel: Obtendo $f(\varepsilon, z)$ a partir de $\hat{f}(\varepsilon, z)$. Para obter um elemento $f(\varepsilon, z) \in\left(J^{(s)}\right)^{-1}(\hat{f})$ para $\hat{f} \in \mathcal{O}[[\varepsilon]]_{s}$, uma maneira efetiva é introduzir a transformada formal de Borel $\left(\hat{\mathcal{B}}_{s} \hat{f}\right)(\eta, z)$ de $\hat{f}(\varepsilon, z)$ definida por

$$
\left(\hat{\mathcal{B}}_{s} \hat{f}\right)(\eta, z):=\sum_{i=0}^{\infty} f_{i}(z) \frac{\eta^{i}}{\Gamma(1+i s)}
$$

onde $\Gamma(n)$ é a função gama de Euler. Pela definição de $\mathcal{O}[[\varepsilon]]_{s},\left(\hat{\mathcal{B}}_{s} \hat{f}\right)(\eta, z)$ é analítica em uma vizinhança de $(\eta, z)=\left(0, z_{0}\right)$, isto é, analítica em $\{|\eta|<r\} \times\left\{\left|z-z_{0}\right|<r\right\}$ para algum $r>0$. Agora, segundo [1], $\hat{f}(\varepsilon, z)$ é s-somável na direção $\theta$ se, e somente se, $\left(\hat{\mathcal{B}}_{s} \hat{f}\right)(\eta, z)$ converge, pode ser analiticamente continuada no setor $S(\theta, \delta ; \infty)$ para algum $\delta>0$, e para constantes $c$ e $K$ suficientemente grandes satisfaz

$$
\left|\left(\hat{\mathcal{B}}_{s} \hat{f}\right)(\eta, z)\right| \leq c \exp \left(K|\eta|^{1 / s}\right), \quad \eta \in S(\theta, \delta ; \infty)
$$


Verificadas essas condições, a $s$-soma de $\hat{f}(\varepsilon, z)$ na direção $\theta$ é dada por

$$
f^{(\theta)}(\varepsilon, z):=\varepsilon^{-1 / s} \int_{0}^{\infty e^{i \theta}} \exp \left(-(\eta / \varepsilon)^{1 / s}\right)\left(\hat{\mathcal{B}}_{s} \hat{f}\right)(\eta, z) d \eta^{1 / s}, \quad|\arg \varepsilon-\theta|<\delta
$$

uma função holomorfa para tais $\varepsilon \operatorname{com} \Re e\left(\varepsilon^{-1 / s} e^{i \theta / s}\right)>K$. Pode ser provado (veja [1]) que $J^{(s)}\left(f^{(\theta)}(\varepsilon, z)\right)=\hat{f}(\varepsilon, z)$ em $S(\theta, \alpha)$, com $\alpha>s \pi$.

\subsection{Normas e Estimativas}

\section{Estimativas Envolvendo a Norma Sup.}

Proposição 3.2.1 Seja $f(z)$ uma função holomorfa sobre $\overline{D_{R}\left(z_{0}\right)}$, e assumamos que $\sup _{z \in \overline{D_{R}\left(z_{0}\right)}}|f(z)| \leq M$. Então

$$
\left|\frac{d^{n} f\left(z_{0}\right)}{d z^{n}}\right| \leq \frac{n ! M}{R^{n}}
$$

para todo $n=0,1,2,3, \ldots$.

Demonstração. Seja $\rho<R$. A fórmula integral de Cauchy nos diz que

$$
\frac{d^{n} f\left(z_{0}\right)}{d z^{n}}=\frac{n !}{2 \pi i} \oint_{\mathcal{C}} \frac{f(w)}{\left(w-z_{0}\right)^{n+1}} d w
$$

$\operatorname{com} \mathcal{C}=\left\{w \in \mathbb{C}:\left|w-z_{0}\right|=\rho\right\}-w=z_{0}+\rho e^{i \theta}$, e portanto $d w=i \rho e^{i \theta} d \theta$. Logo

$$
\begin{aligned}
\left|\frac{d^{n} f\left(z_{0}\right)}{d z^{n}}\right| & \leq \frac{n !}{2 \pi} \oint_{\mathcal{C}} \frac{|f(w)|}{\left|w-z_{0}\right|^{n+1}}|d w| \\
& \leq \frac{n !}{2 \pi} \sup _{w \in \mathcal{C}}|f(w)| \frac{\rho}{\rho^{n+1}} \int_{0}^{2 \pi} d \theta \leq \frac{n ! M}{\rho^{n}}
\end{aligned}
$$

A estimativa (3.2.1) é conseguida tomando-se o limite $\rho \rightarrow R$ em (3.2.3). 
Proposição 3.2.2 Seja $f(z)$ uma função holomorfa sobre $\overline{D_{R}\left(z_{0}\right)}$, e assumamos que $\sup _{z \in \overline{D_{R}\left(z_{0}\right)}}|f(z)| \leq M$. Então

$$
\left|\frac{d f(z)}{d z}\right| \leq \frac{M}{R-r}
$$

vale para todo $z \in \overline{D_{r}\left(z_{0}\right)}$, com $r<R$.

Demonstração. Seja $r<\rho<R$. A fórmula integral de Cauchy nos diz que para cada $z \in \overline{D_{r}\left(z_{0}\right)}$

$$
\frac{d f(z)}{d z}=\frac{1}{2 \pi i} \oint_{\mathcal{C}} \frac{f(w)}{(w-z)^{2}} d w
$$

$\operatorname{com} \mathcal{C}=\{w \in \mathbb{C}:|w-z|=s\}$ e $s=s(z)=\rho-\left|z-z_{0}\right|$ - escolhemos $s$ de tal maneira que $\left|z-z_{0}\right|+s=\rho$. Note que

$$
\rho-r \leq s(z) \leq \rho
$$

Como $w=z+s e^{i \theta}$, e portanto $d w=i s e^{i \theta} d \theta$, segue que

$$
\begin{aligned}
\left|\frac{d f(z)}{d z}\right| & \leq \frac{1}{2 \pi} \oint_{\mathcal{C}} \frac{|f(w)|}{|w-z|^{2}}|d w| \\
& \leq \frac{1}{2 \pi} \sup _{w \in \mathcal{C}}|f(w)| \frac{s}{s^{2}} \int_{0}^{2 \pi} d \theta \\
& \leq \frac{M}{s} \leq \frac{M}{\rho-r}
\end{aligned}
$$

sendo que na última desigualdade utilizamos (3.2.6). Tomando o limite $\rho \rightarrow R$ em (3.2.7), chega-se a (3.2.4). 
Normas de Nagumo. Denotemos por $\mathcal{H}\left(D_{r}\left(z_{0}\right)\right)$ o espaço das funções analíticas sobre $D_{r}\left(z_{0}\right)$ e contínuas em seu fecho $\overline{D_{r}\left(z_{0}\right)}$. Para inteiros não-negativos $k$ definimos a norma de Nagumo de ordem $k$ de $f \in \mathcal{H}\left(D_{r}\left(z_{0}\right)\right)$ por

$$
\|f\|_{k}:=\sup _{z \in D_{r}\left(z_{0}\right)}\left(d_{r}(z)\right)^{k}|f(z)|, \quad \text { com } \quad d_{r}(z)=r-\left|z-z_{0}\right|
$$

Proposição 3.2.3 Para $f, g \in \mathcal{H}\left(D_{r}\left(z_{0}\right)\right)$ e inteiros não-negativos $k, l$, valem as seguintes propriedades:

1. $\|f+g\|_{k} \leq\|f\|_{k}+\|g\|_{k}$;

2. $\|f g\|_{k+l} \leq\|f\|_{k}\|g\|_{l}$;

3. $\left\|f^{\prime}\right\|_{k+1} \leq e(k+1)\|f\|_{k}$;

4. $\|f\|_{k} \leq r\|f\|_{k-1}$;

5. para todo $z \in \overline{D_{r^{\prime}}\left(z_{0}\right)}$, com $r^{\prime}<r,|f(z)| \leq\left(r-r^{\prime}\right)^{-k}\|f\|_{k}$.

Demonstração. Propriedade 1:

$$
\begin{aligned}
\|f+g\|_{k} & =\sup _{z \in D_{r}\left(z_{0}\right)}\left(d_{r}(z)\right)^{k}|f(z)+g(z)| \\
& \leq \sup _{z \in D_{r}\left(z_{0}\right)}\left(d_{r}(z)\right)^{k}(|f(z)|+|g(z)|) \\
& \leq \sup _{z \in D_{r}\left(z_{0}\right)}\left(d_{r}(z)\right)^{k}|f(z)|+\sup _{z \in D_{r}\left(z_{0}\right)}\left(d_{r}(z)\right)^{k}|g(z)|=\|f\|_{k}+\|g\|_{k} .
\end{aligned}
$$

Propriedade 2:

$$
\begin{aligned}
\|f g\|_{k+l} & =\sup _{z \in D_{r}\left(z_{0}\right)}\left(d_{r}(z)\right)^{k+l}|f(z) g(z)| \\
& \leq \sup _{z \in D_{r}\left(z_{0}\right)}\left(d_{r}(z)\right)^{k+l}(|f(z)||g(z)|) \\
& \leq \sup _{z \in D_{r}\left(z_{0}\right)}\left(d_{r}(z)\right)^{k}|f(z)| \sup _{z \in D_{r}\left(z_{0}\right)}\left(d_{r}(z)\right)^{l}|g(z)|=\|f\|_{k}\|g\|_{l} .
\end{aligned}
$$


Propriedade 3: a fórmula integral de Cauchy nos diz que para cada $z \in D_{r}\left(z_{0}\right)$

$$
f^{\prime}(z)=\frac{1}{2 \pi i} \oint_{\mathcal{C}} \frac{f(w)}{(w-z)^{2}} d w
$$

$\operatorname{com} \mathcal{C}=\{w \in \mathbb{C}:|w-z|=s\}$ e $s=s(z)$ tal que $\left|z-z_{0}\right|+s<r$. Então

$$
\begin{aligned}
\left|f^{\prime}(z)\right| & \leq \sup _{w \in \mathcal{C}}|f(w)| \frac{1}{s} \\
& \leq \sup _{w \in \mathcal{C}}\left(d_{r}(w)\right)^{-k}\left(\sup _{w \in \mathcal{C}}\left(d_{r}(w)\right)^{k}|f(w)|\right) \frac{1}{s} \\
& \leq\left(d_{r}(z)-s\right)^{-k}\|f\|_{k} \frac{1}{s},
\end{aligned}
$$

sendo que na última desigualdade usamos o fato que

$$
d_{r}(w)=r-\left|w-z_{0}\right| \geq r-\left|z-z_{0}\right|-|w-z|=d_{r}(z)-s .
$$

Para $k>0$, escolhendo $s(z)=\frac{d_{r}(z)}{k+1}$ e utilizando a desigualdade $\left(1-\frac{1}{1+k}\right)^{-k}<e$, segue que

$$
\begin{aligned}
\left|f^{\prime}(z)\right| & \leq\left(d_{r}(z)\right)^{-(k+1)}\left(1-\frac{1}{k+1}\right)^{-k}\|f\|_{k}(k+1) \\
& \leq\left(d_{r}(z)\right)^{-(k+1)} e(k+1)\|f\|_{k} .
\end{aligned}
$$

Conclui-se portanto que $\left(d_{r}(z)\right)^{k+1}\left|f^{\prime}(z)\right| \leq e(k+1)\|f\|_{k}$, de onde segue imediatamente a propriedade 3.

Propriedade 4:

$$
\begin{aligned}
\|f\|_{k} & =\sup _{z \in D_{r}\left(z_{0}\right)}\left(d_{r}(z)\right)^{k}|f(z)| \\
& \leq \sup _{z \in D_{r}\left(z_{0}\right)} d_{r}(z) \sup _{z \in D_{r}\left(z_{0}\right)}\left(d_{r}(z)\right)^{k-1}|f(z)|=r\|f\|_{k-1} .
\end{aligned}
$$


Propriedade 5: essa segue do fato que para todo $z \in D_{r}\left(z_{0}\right)$ temos

$$
\left(d_{r}(z)\right)^{k}|f(z)| \leq \sup _{z \in D_{r}\left(z_{0}\right)}\left(d_{r}(z)\right)^{k}|f(z)|=\|f\|_{k}
$$

e do fato que para todo $z \in \overline{D_{r^{\prime}}\left(z_{0}\right)}$ (sendo $r^{\prime}<r$ )

$$
d_{r}(z)=r-\left|z-z_{0}\right| \geq r-r^{\prime}
$$

Assim encerramos a demonstração.

Neste trabalho usamos outra propriedade que torna $\mathcal{X}_{r, k}=\left(\mathcal{H}\left(D_{r}\left(z_{0}\right)\right),\|\cdot\|_{k}\right)$ uma escala de espaços de Banach. Note que $f \in \mathcal{X}_{r, k}$ implica que $f \in \mathcal{X}_{r, l}$ para todo $l>k$ e

$$
\|f\|_{l} \leq r^{l-k}\|f\|_{k} \leq\|f\|_{k}
$$

contanto que $r \leq 1$. Sob tal condição, $\mathcal{X}_{r, k}$ é um subespaço de $\mathcal{X}_{r, l}$, a injeção $\mathcal{X}_{r, k} \longrightarrow \mathcal{X}_{r, l}$ é contínua e tem norma $\leq 1$.

Modificação das Normas de Nagumo. Consideremos agora uma função $f(\varepsilon, z)$ holomorfa em $S(\theta, \alpha ; E) \times D_{r}\left(z_{0}\right)$, e sua expansão

$$
f(\varepsilon, z)=\sum_{n=0}^{\infty} a_{n}(\varepsilon)\left(z-z_{0}\right)^{n}
$$

Para inteiros não negativos $k$, definimos a modificação das normas de Nagumo por

$$
\|f\|_{k}:=\sup _{z \in D_{r}\left(z_{0}\right)}\left(d_{r}(z)\right)^{k} \sum_{n=0}^{\infty} \sup _{\varepsilon \in S(\theta, \alpha ; E)}\left|a_{n}(\varepsilon)\right|\left|z-z_{0}\right|^{n} .
$$

Com essa definição, as propriedades listadas no parágrafo anterior para as normas de Nagumo ainda permanecem válidas (vide [3]). 


\subsection{Ingredientes Chave}

\section{Coeficientes para Controlar Convoluções.}

Proposição 3.3.1 Seja $\lambda \geq 0$ qualquer e A um número real positivo tal que

$$
A \leq \frac{1}{2}\left(1+\frac{\pi^{2}}{3}\right)^{-1}=0,1165536 \ldots
$$

Consideremos a seqüência $\left(C_{l}\right)_{l=0}^{m} \operatorname{com} C_{0}=A$ e

$$
C_{l}=\frac{A l !^{\lambda}}{l^{2}} \quad \forall l \geq 1
$$

Então

$$
\sum_{l=0}^{m} C_{l} C_{m-l} \leq C_{m} \quad \forall m \geq 0 .
$$

Demonstração. Uma vez que $\left(\begin{array}{c}m \\ l\end{array}\right) \geq 1$,

$$
\frac{m}{l(m-l)}=\frac{1}{l}+\frac{1}{m-l}
$$

e $0 \leq(a-b)^{2}=2\left(a^{2}+b^{2}\right)-(a+b)^{2}$ vale para quaisquer números reais $a$ e $b$, fazendo $a=1 / l$ e $b=1 /(m-l)$ temos

$$
\begin{aligned}
\frac{1}{C_{m}} \sum_{l=0}^{m} C_{l} C_{m-l} & =A\left(2+\sum_{l=1}^{m-1}\left(\begin{array}{c}
m \\
l
\end{array}\right)^{-\lambda} \frac{m^{2}}{l^{2}(m-l)^{2}}\right) \\
& \leq A\left(2+\sum_{l=1}^{m-1} \frac{m^{2}}{l^{2}(m-l)^{2}}\right) \\
& \leq 2 A\left(1+\sum_{l=1}^{m-1}\left(\frac{1}{l^{2}}+\frac{1}{(m-l)^{2}}\right)\right) \leq 2 A\left(1+\frac{\pi^{2}}{3}\right) \leq 1
\end{aligned}
$$

sendo que na última desigualdade utilizamos a hipótese que $A$ satifaz (3.3.1). 
Por indução, segue de (3.3.3) que para qualquer $1 \leq k \leq m$

$$
\sum_{\substack{l_{1}, \ldots, l_{k} \geq 1: \\ l_{1}+\cdots+l_{k}=m}} C_{l_{1}} \cdots C_{l_{k}} \leq C_{m}
$$

Fórmula de Scott. Uma fórmula alternativa à fórmula de Faà di Bruno para derivadas de ordem superior de composições é devida a Scott (vide referência [18]). Denotando por $h^{(j)}(x)$ a derivada $j$-ésima de $h$ (com respeito a $x$ ), enunciamos:

Proposição 3.3.2 Sejam $X, Y$ e $Z$ abertos de $\mathbb{C}$. Sejam $g: X \rightarrow Y$ e $f: Y \rightarrow Z$ funções analíticas. Então, para cada $j \geq 1$ vale

$$
\frac{1}{j !}(f \circ g)^{(j)}(x)=\sum_{k=1}^{j} \frac{1}{k !} f^{(k)} \circ g(x)\left[\left.\frac{1}{j !} \frac{d^{j}}{d x^{j}}(g(x))^{k}\right|_{g(x)=0}\right] \text {. }
$$

Demonstração. Tomemos um ponto $x_{0} \in X$ arbitrário e o fixemos. Para $\delta \in \mathbb{C}$ tal que $x_{0}+\delta \in X$, seja

$$
u(\delta)=g\left(x_{0}+\delta\right)-g\left(x_{0}\right) .
$$

Pela fórmula de Taylor, com $n \geq 1$ qualquer, temos que

$$
\begin{aligned}
(f \circ g)\left(x_{0}+\delta\right)-(f \circ g)\left(x_{0}\right) & =f\left(u(\delta)+g\left(x_{0}\right)\right)-f\left(g\left(x_{0}\right)\right) \\
& =\sum_{k=1}^{n} \frac{1}{k !} f^{(k)}\left(g\left(x_{0}\right)\right) u^{k}(\delta)+o\left(u^{n}(\delta)\right) .
\end{aligned}
$$

Definamos $U_{k}(\delta):=u^{k}(\delta)$. Para $|\delta|$ pequeno façamos a expansão de Taylor

$$
U_{k}(\delta)=\sum_{j=k}^{n} \frac{1}{j !} U_{k}^{(j)}(0) \delta^{j}+o\left(\delta^{n}\right)
$$

- note que $U_{k}^{(j)}(0)=0$ para $j=0,1,2, \ldots, k-1$ : 


$$
\begin{aligned}
\frac{1}{j !} U_{k}^{(j)}(0) & =\left.\frac{1}{j !} \frac{d^{j}}{d \delta^{j}} u^{k}(\delta)\right|_{\delta=0} \\
& =\left.\frac{1}{j !} \frac{d^{j}}{d \delta^{j}}\left(g\left(x_{0}+\delta\right)-g\left(x_{0}\right)\right)^{k}\right|_{\delta=0} \\
& =\left.\frac{1}{j !} \frac{d^{j}}{d x_{0}^{j}}\left(g\left(x_{0}\right)\right)^{k}\right|_{g\left(x_{0}\right)=0} .
\end{aligned}
$$

Substituindo (3.3.8) em (3.3.7) segue que

$$
\begin{gathered}
(f \circ g)\left(x_{0}+\delta\right)-(f \circ g)\left(x_{0}\right)=\sum_{k=1}^{n} \frac{1}{k !} f^{(k)}\left(g\left(x_{0}\right)\right) \sum_{j=k}^{n} \frac{1}{j !} U_{k}^{(j)}(0) \delta^{j}+o\left(\delta^{n}\right) \\
=\sum_{j=1}^{n}\left(\sum_{k=1}^{j} \frac{1}{k !} f^{(k)}\left(g\left(x_{0}\right)\right) \frac{1}{j !} U_{k}^{(j)}(0)\right) \delta^{j}+o\left(\delta^{n}\right) .
\end{gathered}
$$

Por outro lado, se aplicarmos a fórmula de Taylor diretamente à $f \circ g$ temos

$$
(f \circ g)\left(x_{0}+\delta\right)-(f \circ g)\left(x_{0}\right)=\sum_{j=1}^{n} \frac{1}{j !}(f \circ g)^{(j)}\left(x_{0}\right) \delta^{j}+o\left(\delta^{n}\right) .
$$

Subtraindo (3.3.10) de (3.3.11), vemos que

$$
\sum_{j=1}^{n}\left(\frac{1}{j !}(f \circ g)^{(j)}\left(x_{0}\right)-\sum_{k=1}^{j} \frac{1}{k !} f^{(k)}\left(g\left(x_{0}\right)\right) \frac{1}{j !} U_{k}^{(j)}(0)\right) \delta^{j}=o\left(\delta^{n}\right) .
$$

Através do processo "dividir ambos os lados de (3.3.12) por $\delta$ e tomar $\delta \rightarrow 0$ ", concluímos que a igualdade

$$
\frac{1}{j !}(f \circ g)^{(j)}\left(x_{0}\right)=\sum_{k=1}^{j} \frac{1}{k !} f^{(k)}\left(g\left(x_{0}\right)\right) \frac{1}{j !} U_{k}^{(j)}(0)
$$

vale para cada $j=1,2, \ldots, n$. Por (3.3.9), e dos fatos que $x_{0}$ é um ponto qualquer de $X$ e $n \geq 1$ qualquer, segue o resultado enunciado. 
Fazendo uso da regra de Leibniz

$$
\frac{1}{j !} \frac{d^{j}}{d x^{j}}\left(g_{1}(x) \cdots g_{k}(x)\right)=\sum_{\substack{l_{1}, \ldots, l_{k} \geq 0: \\ l_{1}+\cdots+l_{k}=j}} \frac{1}{l_{1} !} g_{1}^{\left(l_{1}\right)}(x) \cdots \frac{1}{l_{k} !} g_{k}^{\left(l_{k}\right)}(x)
$$

escrevemos

$$
\frac{1}{j !}(f \circ g)^{(j)}(x)=\sum_{k=1}^{j} \frac{1}{k !} f^{(k)} \circ g(x) \sum_{\substack{l_{1}, \ldots, l_{k} \geq 1: \\ l_{1}+\cdots+l_{k}=j}} \frac{1}{l_{1} !} g^{\left(l_{1}\right)}(x) \cdots \frac{1}{l_{k} !} g^{\left(l_{k}\right)}(x) .
$$

Série Majorante. Diz-se que a série $\sum_{n=0}^{\infty} c_{n}\left(z-z_{0}\right)^{n}$ é majorada (denotado por $<<$ ) pela série $\sum_{n=0}^{\infty} C_{n}\left(z-z_{0}\right)^{n}$ de coeficientes não-negativos se $\left|c_{n}\right| \leq C_{n}$ para todo $n \geq 0$.

Proposição 3.3.3 Sejam

$$
f(z)=\sum_{n=0}^{\infty} c_{n}\left(z-z_{0}\right)^{n}<<F(z)=\sum_{n=0}^{\infty} C_{n}\left(z-z_{0}\right)^{n}
$$

e

$$
g(z)=\sum_{n=0}^{\infty} d_{n}\left(z-z_{0}\right)^{n}<<G(z)=\sum_{n=0}^{\infty} D_{n}\left(z-z_{0}\right)^{n} .
$$

Seja $h(z)=\sum_{n=0}^{\infty} e_{n}\left(z-z_{0}\right)^{n}$ tal que $e_{0}>0$ e $e_{n} \geq 0$ para todo $n \geq 1$. Então:

1. $f(z)+g(z)<<F(z)+G(z)$;

2. $f(z) g(z)<<F(z) G(z)$;

3. $f^{\prime}(z)<<F^{\prime}(z)$;

4. $\frac{-1}{h(z)}<<\frac{-1}{h(z)-2 e_{0}}$.

Demonstração. Propriedade 1: Dado que $\left|c_{n}+d_{n}\right| \leq\left|c_{n}\right|+\left|d_{n}\right| \leq C_{n}+D_{n}$ vale para todo $n \geq 0$, segue que 


$$
f(z)+g(z)=\sum_{n=0}^{\infty}\left(c_{n}+d_{n}\right)\left(z-z_{0}\right)^{n}<<F(z)+G(z)=\sum_{n=0}^{\infty}\left(C_{n}+D_{n}\right)\left(z-z_{0}\right)^{n} .
$$

Propriedade 2: Multiplicando as duas séries, temos

$$
\begin{aligned}
f(z) g(z) & =\sum_{l=0}^{\infty} c_{l}\left(z-z_{0}\right)^{l} \sum_{m=0}^{\infty} d_{m}\left(z-z_{0}\right)^{m} \\
& =\sum_{n=0}^{\infty}\left(\sum_{m=0}^{n} c_{m} d_{n-m}\right)\left(z-z_{0}\right)^{n} .
\end{aligned}
$$

Vemos dessa expressão que o $n$-ésimo coeficiente da série $f(z) g(z)$ satisfaz, para cada $n \geq 0$,

$$
\begin{aligned}
\left|\sum_{m=0}^{n} c_{m} d_{n-m}\right| & \leq \sum_{m=0}^{n}\left|c_{m}\right|\left|d_{n-m}\right| \\
& \leq \sum_{m=0}^{n} C_{m} D_{n-m} .
\end{aligned}
$$

A propriedade 2 está assim mostrada, pois a última soma é o $n$-ésimo coeficiente da série $F(z) G(z)$ :

$$
\begin{aligned}
F(z) G(z) & =\sum_{l=0}^{\infty} C_{l}\left(z-z_{0}\right)^{l} \sum_{m=0}^{\infty} D_{m}\left(z-z_{0}\right)^{m} \\
& =\sum_{n=0}^{\infty}\left(\sum_{m=0}^{n} C_{m} D_{n-m}\right)\left(z-z_{0}\right)^{n} .
\end{aligned}
$$

Propriedade 3: A desigualdade $(n+1)\left|c_{n+1}\right| \leq(n+1) C_{n+1}$ vale para todo $n \geq 0$. Logo

$$
f^{\prime}(z)=\sum_{n=0}^{\infty}(n+1) c_{n+1}\left(z-z_{0}\right)^{n}<<F^{\prime}(z)=\sum_{n=0}^{\infty}(n+1) C_{n+1}\left(z-z_{0}\right)^{n} .
$$


Propriedade 4: Seja $\frac{-1}{h(z)}=(r \circ h)(z), \operatorname{com} r(x):=\frac{-1}{x}$, e consideremos a série

$$
\frac{-1}{h(z)}=\sum_{n=0}^{\infty} \lambda_{n}\left(z-z_{0}\right)^{n}
$$

Temos que

$$
\lambda_{0}=\left.\frac{-1}{h(z)}\right|_{z=z_{0}}=\frac{-1}{e_{0}}
$$

e para $n \geq 1$

$$
\begin{aligned}
\lambda_{n} & =\left.\frac{1}{n !} \frac{d^{n}}{d z^{n}}\left(\frac{-1}{h(z)}\right)\right|_{z=z_{0}}=\left.\frac{1}{n !}(r \circ h)^{(n)}(z)\right|_{z=z_{0}} \\
& =\sum_{k=1}^{n} \frac{(-1)^{k+1}}{\left(e_{0}\right)^{k+1}} \sum_{\substack{l_{1}, \ldots, l_{k} \geq 1: \\
l_{1}+\cdots+l_{k}=n}} e_{l_{1}} \cdots e_{l_{k}}
\end{aligned}
$$

pela fórmula de $\operatorname{Scott}(3.3 .15)$. Como $e_{0}>0$ e $e_{n} \geq 0$ para todo $n \geq 1$,

$$
\left|\lambda_{0}\right|=\frac{-1}{-e_{0}} \equiv \Lambda_{0}
$$

e

$$
\left|\lambda_{n}\right| \leq \sum_{k=1}^{n} \frac{(-1)^{k+1}}{\left(-e_{0}\right)^{k+1}} \sum_{\substack{l_{1}, \ldots, l_{k} \geq 1: \\ l_{1}+\cdots+l_{k}=n}} e_{l_{1}} \cdots e_{l_{k}} \equiv \Lambda_{n}
$$

Vemos portanto que

$$
\frac{-1}{h(z)}=\sum_{n=0}^{\infty} \lambda_{n}\left(z-z_{0}\right)^{n}<<\frac{-1}{H(z)}=\sum_{n=0}^{\infty} \Lambda_{n}\left(z-z_{0}\right)^{n}
$$

$\operatorname{com} H(z)=-e_{0}+\sum_{n=1}^{\infty} e_{n}\left(z-z_{0}\right)^{n}=h(z)-2 e_{0}$.

O modo como serão utilizadas séries majorantes é apresentado pelo seguinte resultado:

Proposição 3.3.4 Suponhamos que $\psi(z)=\sum_{n=0}^{\infty} c_{n}\left(z-z_{0}\right)^{n}<<\sum_{n=0}^{\infty} C_{n}\left(z-z_{0}\right)^{n}$ e que a série $\sum_{n=0}^{\infty} C_{n}\left(z-z_{0}\right)^{n}$ é convergente para todo $z \in D_{r}\left(z_{0}\right)$. Então $\psi(z)$ é uma função analítica em $D_{r}\left(z_{0}\right)$. 
Demonstração. Por hipótese, a série numérica $\sum_{n=0}^{\infty} C_{n} \sigma^{n}$ é convergente para todo $\sigma<r$. Pela hipótese de majoração temos que

$$
\left|c_{n}\right|\left|z-z_{0}\right|^{n} \leq C_{n} \sigma^{n} \equiv M_{n}
$$

vale para cada $n \geq 0$ e todo $z \in \overline{D_{\sigma}\left(z_{0}\right)}$. Segue pelo Teste $M$ de Weierstrass (vide Teorema 3.3.5 a seguir enunciado) que a série $\sum_{n=0}^{\infty} c_{n}\left(z-z_{0}\right)^{n}$ converge uniformemente sobre $\overline{D_{\sigma}\left(z_{0}\right)}, \sigma<r$. Portanto, definindo

$$
\psi_{m}(z):=\sum_{n=0}^{m} c_{n}\left(z-z_{0}\right)^{n}
$$

temos que $\left(\psi_{m}(z)\right)_{m \geq 0}$, uma seqüência de funções analíticas em $D_{r}\left(z_{0}\right)$, é tal que $\psi_{m}(z)$ converge uniformemente para $\psi(z)=\sum_{n=0}^{\infty} c_{n}\left(z-z_{0}\right)^{n}$ sobre todo compacto $\overline{D_{\sigma}\left(z_{0}\right)}$ de $D_{r}\left(z_{0}\right)$ quando $m \rightarrow \infty$. Conclui-se pelo Teorema 3.3.6 (a seguir enunciado) que $\psi(z)$ é uma função analítica em $D_{r}\left(z_{0}\right)$.

Teorema 3.3.5 (Teste $\mathbf{M}$ de Weierstrass) Seja $\sum_{n=0}^{\infty} f_{n}(z)$ uma série de funções $f_{n}$ : $\Omega \rightarrow \mathbb{K}$, com $\Omega \subset \mathbb{K}$ e $\mathbb{K}=\mathbb{R}$ ou $\mathbb{K}=\mathbb{C}$. Suponhamos que existam constantes $M_{n} \geq 0$ tais que

$$
\left|f_{n}(z)\right| \leq M_{n}
$$

para n's suficientemente grandes e todo $z \in \Omega$, e que a série numérica $\sum_{n=0}^{\infty} M_{n}$ seja convergente. Então a série de funções $\sum_{n=0}^{\infty} f_{n}(z)$ converge uniforme e absolutamente sobre $\Omega$.

Teorema 3.3.6 (Weierstrass) Seja $\Omega$ um aberto de $\mathbb{C}^{n}$ e $\left(\psi_{m}(z)\right)_{m \geq 0}$ uma seqüência de funções analíticas em $\Omega$ tal que $\psi_{m}(z)$ converge uniformemente para $\psi(z)$ nas partes compactas de $\Omega$ quando $m \rightarrow \infty$. Então $\psi(z)$ é uma função analítica em $\Omega$.

Séries majorantes e a Proposição 3.3.4 são utilizadas no contexto de equações diferenciais para mostrar a existência de uma única solução analítica: trata-se do chamado método majorante de Cauchy - vide referência [19]. 


\subsection{Propriedades de $g(\varepsilon, w)$ a Partir das Proprie- dades de $f(\varepsilon, z)=g^{-1}(\varepsilon, z)$}

Nesta seção enunciamos e provamos nosso primeiro teorema. Ele trata das propriedades que podem ser inferidas para uma função $g(\varepsilon, w)$ a partir das propriedades verificadas para sua inversa com respeito à variável $w, f(\varepsilon, z)=g^{-1}(\varepsilon, z)$. Mais especificamente, as propriedades de ser analítica em $S \times D$ e Gevrey ordem $s$ assintoticamente expansiva em $S, S$ um setor e $D$ um disco do plano complexo.

Teorema 3.4.1 Seja $f(\varepsilon, z)$ uma função analítica em $S(\theta, \alpha ; E) \times D_{R}\left(z_{0}\right)$. Suponha:

1. $f(\varepsilon, z) \neq f\left(\varepsilon, z_{0}\right)$ vale para todo $z \in D_{R}\left(z_{0}\right) \backslash\left\{z_{0}\right\}$ e $\varepsilon \in S(\theta, \alpha ; E)$;

2. $f_{z}\left(\varepsilon, z_{0}\right) \neq 0$ para cada $\varepsilon \in S(\theta, \alpha ; E)$.

Sejam $r, \rho$ e $E_{1}$ números reais tais que $0<\rho<r<R$ e $E_{1}<E$. Suponha que

$$
m(\varepsilon, \rho) \equiv \min _{0 \leq \theta<2 \pi}\left|f\left(\varepsilon, z_{0}+\rho e^{i \theta}\right)\right|>0
$$

para cada $\varepsilon \in S\left(\theta, \alpha ; E_{1}\right), e$

$$
\left|\frac{1}{i !} \frac{\partial^{i} f(\varepsilon, z)}{\partial \varepsilon^{i}}\right| \leq C i !^{s} \mu^{i}
$$

para cada $i \geq 0$ e $(\varepsilon, z) \in S\left(\theta, \alpha ; E_{1}\right) \times \overline{D_{r}\left(z_{0}\right)}$. Então $g(\varepsilon, w)=f^{-1}(\varepsilon, w)$ é uma função analítica em $S\left(\theta, \alpha ; E_{1}\right) \times D_{m_{1}(\rho)}(0), \operatorname{com} m_{1}(\rho)=\min _{\varepsilon \in S\left(\theta, \alpha ; E_{1}\right)} m(\varepsilon, \rho)$, e satisfaz

$$
\left|\frac{1}{i !} \frac{\partial^{i} g(\varepsilon, w)}{\partial \varepsilon^{i}}\right| \leq \widetilde{C} i !^{s} \tilde{\mu}^{i}
$$

para cada $i \geq 0$ e $(\varepsilon, w) \in S\left(\theta, \alpha ; E_{1}\right) \times \overline{D_{m_{2}(\rho)}(0)}, \operatorname{com}_{2}(\rho)<m_{1}(\rho)$.

Comentário 3.4.2 As hipóteses 1. e 2. são condições de univalência local, em que $f(\varepsilon, z)-f\left(\varepsilon, z^{\prime}\right) \neq 0$ para todo $z \neq z^{\prime}$ em $D_{R}\left(z_{0}\right)$, para algum $R>0$ e todo $\varepsilon \in S(\theta, \alpha ; E)$ (mesmo quando $\varepsilon$ se aproxima de 0 ). 
Pelo Teorema de Taylor

$$
r_{I}(\varepsilon, z)=\varepsilon^{-I}\left(f(\varepsilon, z)-\sum_{i=0}^{I-1} f_{i}(z) \varepsilon^{i}\right)=\frac{I}{\varepsilon^{I}} \int_{0}^{\varepsilon} \frac{1}{I !} \frac{\partial^{I} f(\xi, z)}{\partial \xi^{I}}(\varepsilon-\xi)^{I-1} d \xi
$$

sendo que a integral é efetuada ao longo de um caminho interior a $S\left(\theta, \alpha ; E_{1}\right)$ que parte de 0 e vai a $\varepsilon$. Assim, (3.4.2) nos diz que

$$
\max _{z \in \overline{D_{r}\left(z_{0}\right)}}\left|r_{I}(\varepsilon, z)\right| \leq C I !^{s} \mu^{I}
$$

vale para $I=1,2,3, \ldots$ e todo $\varepsilon \in S^{\prime}$, com $S^{\prime}$ qualquer subsetor próprio de $S\left(\theta, \alpha ; E_{1}\right)$. Se adicionalmente $f(\varepsilon, z)$ e as derivadas parciais $\partial^{i} f(\varepsilon, z) / \partial \varepsilon^{i}, i \geq 1$, são contínuas quando $\varepsilon \rightarrow 0$ em $S\left(\theta, \alpha ; E_{1}\right)$, temos que (3.4.2) vale no limite $\varepsilon \rightarrow 0$; conclui-se que $f(\varepsilon, z)$ é uma função Gevrey ordem $s$ assintoticamente expansiva em $S\left(\theta, \alpha ; E_{1}\right)$ (vide definições 3.1.1 e 3.1.2): existe uma série formal

$$
\hat{f}(\varepsilon, z)=\sum_{i=0}^{\infty} a_{i}(z) \varepsilon^{i},
$$

um elemento de $\mathcal{O}(r)[[\varepsilon]]_{s}$, que é a expansão assintótica de Gevrey (ordem $s$ ) de $f(\varepsilon, z)$ quando $\varepsilon \rightarrow 0$ em $S\left(\theta, \alpha ; E_{1}\right)$.

Comentário 3.4.3 Se $f(\varepsilon, z)$ e as derivadas parciais $\partial^{i} f(\varepsilon, z) / \partial \varepsilon^{i}, i \geq 1$, são contínuas quando $\varepsilon \rightarrow 0 \mathrm{em} S\left(\theta, \alpha ; E_{1}\right)$, então da demonstração do Teorema 3.4.1 segue que a estimativa (3.4.3) vale quando $\varepsilon$ vai a zero em $S\left(\theta, \alpha ; E_{1}\right)$. Pela discussão apresentada acima, segue que $g(\varepsilon, w)$ é uma função Gevrey ordem s assintoticamente expansiva em $S\left(\theta, \alpha ; E_{1}\right)$ : existe uma série formal

$$
\hat{g}(\varepsilon, w)=\sum_{i=0}^{\infty} b_{i}(w) \varepsilon^{i},
$$

um elemento de $\mathcal{O}\left(m_{2}\right)[[\varepsilon]]_{s}$, que é a expansão assintótica de Gevrey (ordem s) de $g(\varepsilon, w)$ quando $\varepsilon \rightarrow 0$ em $S\left(\theta, \alpha ; E_{1}\right)$. 
Para demonstrarmos o Teorema 3.4.1 precisamos de algumas definições e resultados: As definições a seguir apresentadas encontram-se na Seção 4 do Capítulo II de [26]; os teoremas 3.4.6 e 3.4.7 estão na Seção 9.2 do Capítulo IX de [26] (eles são, respectivamente, os teoremas 9.2.3 e 9.2.4 dessa referência); o terceiro teorema está exposto Seção 9.4 do Capítulo IX de [26] (Teorema 9.4.4).

\section{Duas Definições.}

Definição 3.4.4 (Curva retificável) Seja $0=t_{0}<t_{1}<\ldots<t_{n}=1$ qualquer partição do intervalo $[0,1]$. A essa partição está associada a soma

$$
S_{\pi}=\sum_{k=1}^{n}\left|\gamma\left(t_{k}\right)-\gamma\left(t_{k-1}\right)\right| .
$$

A curva $\Gamma: \gamma=\gamma(t)$ é dita ser de comprimento finito, ou ser retificável, se o conjunto $\left\{S_{\pi}\right\}$ de tais somas é limitado; o comprimento de $\Gamma$ é por definição

$$
l(\Gamma)=\sup S_{\pi} .
$$

Definição 3.4.5 (Scroc) Uma curva $\mathcal{C}$ é dita ser um "scroc" se é uma curva simples fechada retificável e orientada.

Dois Teoremas. Seja $\mathcal{C}_{i}$ a região interior a uma curva fechada $\mathcal{C}$; a seguir denotaremos por $\mathcal{C}^{*}$ o conjunto $\mathcal{C} \cup \mathcal{C}_{i}$, e por $Z_{f}\left(P_{f}\right)$ o número de zeros (pólos) de uma função $f$ meromorfa em $\mathcal{C}_{i}$.

Teorema 3.4.6 (Teorema de Rouché) Seja C um "scroc" e $f(z)$ uma função meromorfa em $\mathcal{C}^{*}$, mas que não possui zeros nem pólos sobre $\mathcal{C}$. Suponha que $f(z)$ pode ser escrita como a soma de duas funções meromorfas em $\mathcal{C}^{*}$

$$
f(z)=g(z)+h(z)
$$

tal que $g(z) \neq 0, \infty$ sobre $\mathcal{C}$. Além disso, suponha que 


$$
|g(z)|>|h(z)|
$$

sobre $\mathcal{C}$. Então a mudança no argumento de $f(z)$ quando z percorre $\mathcal{C}$ é a mesma mudança que ocorre no argumento de $g(z)$, e a diferença entre o número de zeros $e$ pólos é a mesma para essas duas funções:

$$
Z_{f}-P_{f}=Z_{g}-P_{g}
$$

Outro teorema a ser utilizado é:

Teorema 3.4.7 Seja $f(z)$ uma função meromorfa em $\mathcal{C}^{*}$ com zeros em $a_{1}, a_{2}, \ldots, a_{n}$ e pólos em $b_{1}, b_{2}, \ldots, b_{m}$, sendo que nenhum deles se encontra sobre $\mathcal{C}$. Seja $g(z)$ uma função meromorfa em $\mathcal{C}^{*}$. Então

$$
\frac{1}{2 \pi i} \int_{\mathcal{C}} g(z) \frac{f^{\prime}(z)}{f(z)} d z=\sum_{j=1}^{n} g\left(a_{j}\right)-\sum_{k=1}^{m} g\left(b_{k}\right)
$$

sendo que cada zero e cada pólo aparece na soma o número de vezes correspondente à sua multiplicidade.

Estamos agora em posição de provar o Teorema 3.4.1:

Demonstração. Sem perda de generalidade, assumamos que $f\left(\varepsilon_{0}, z_{0}\right)=0$ para algum $\varepsilon_{0} \in S(\theta, \alpha ; E)$. As hipóteses 1. e 2. para $\varepsilon=\varepsilon_{0}$ são as requeridas pelo Teorema da Função Implícita Analítica (Teorema 9.4.4 de [26]) - trata-se de condições de univalência local, em que $f(\varepsilon, z)-f\left(\varepsilon, z^{\prime}\right) \neq 0$ para todo $z \neq z^{\prime}$ em $D_{R}\left(z_{0}\right)$, para algum $R>0$ e todo $\varepsilon \in S(\theta, \alpha ; E)$ (mesmo quando $\varepsilon$ se aproxima de 0 ). Por esse teorema existe uma e somente uma solução $z=\phi(\varepsilon)$ de $f(\varepsilon, z)=0$, em uma vizinhança $U$ de $\left(\varepsilon_{0}, z_{0}\right)$, que é uma função analítica de $\varepsilon$. Uma vez que $z_{0}=\phi\left(\varepsilon_{0}\right)$, existe um disco $D_{e}\left(\varepsilon_{0}\right)$ centrado em $\varepsilon_{0}$ e de raio $e>0$ em $S(\theta, \alpha ; E)$ tal que, para cada $\varepsilon \in D_{e}\left(\varepsilon_{0}\right), \overline{D_{r}(\phi(\varepsilon))} \subset D_{R}\left(z_{0}\right)$ para algum $0<r<R$. Do fato que as hipóteses 1. e 2. são uniformes em $\varepsilon_{0} \in S(\theta, \alpha ; E)$, $\phi(\varepsilon)$ pode ser estendida para todo $\varepsilon \in S(\theta, \alpha ; E)$ e podemos escolher $r>0$ independente de $\varepsilon$ em $S\left(\theta, \alpha ; E_{1}\right)$ para algum $E_{1}<E$. 
Agora, para um $w$ fixo em $D_{m(\varepsilon, \rho)}(0)$ e para $z$ sobre $\mathcal{C}:=\left\{z \in \mathbb{C}:\left|z-z_{0}\right|=\rho\right\}$, com $\rho<r$ e $m(\varepsilon, \rho)$ definida em (3.4.1), temos

$$
|f(\varepsilon, z)| \geq m(\varepsilon, \rho)>|w|
$$

Pelo Teorema 3.4.6, isso implica que as duas funções analíticas

$$
f(\varepsilon, z) \text { e } f(\varepsilon, z)-w
$$

possuem o mesmo número de zeros no interior de $\mathcal{C}$. Uma vez que $f(\varepsilon, z)$ tem um único zero para cada $\varepsilon \in D_{e}\left(\varepsilon_{0}\right)$, a saber em $z=\phi(\varepsilon)$, concluímos que a equação

$$
f(\varepsilon, z)=w
$$

tem uma única raíz, digamos $z=g(\varepsilon, w)$, no interior de $\mathcal{C}$. Pelo Teorema 3.4.7 essa raíz é dada pela fórmula

$$
g(\varepsilon, w)=\frac{1}{2 \pi i} \int_{\mathcal{C}} \frac{z f_{z}(\varepsilon, z)}{f(\varepsilon, z)-w} d z
$$

Para vermos que $g(\varepsilon, w)$ é uma função analítica em $(\varepsilon, w) \in S\left(\theta, \alpha ; E_{1}\right) \times D_{m_{1}}(0)$, com $m_{1}=m_{1}(\rho)$ como definida no enunciado, é suficiente que se note que

$$
\frac{1}{f(\varepsilon, z)-w}=\sum_{n=0}^{\infty} \frac{1}{(f(\varepsilon, z))^{n+1}} w^{n}
$$

Essa série converge uniformemente com respeito a $\varepsilon, z$ e $w$ desde que $\varepsilon \in S\left(\theta, \alpha ; E_{1}\right)$, $z \in \mathcal{C}$ e $|w| \leq(1-\epsilon) m(\varepsilon, \rho), \epsilon>0$, e ela pode ser multiplicada pela função limitada $z f_{z}(\varepsilon, z)$ e integrada termo a termo. O resultado é

$$
g(\varepsilon, w)=\sum_{n=0}^{\infty} w^{n} \frac{1}{2 \pi i} \int_{\mathcal{C}} \frac{z f_{z}(\varepsilon, z)}{(f(\varepsilon, z))^{n+1}} d z
$$

Note que $g(\varepsilon, w)$ é contínua quando $\varepsilon$ tende a zero em $S\left(\theta, \alpha ; E_{1}\right)$ se $f(\varepsilon, z)$ é contínua quando $\varepsilon$ tende a zero em $S\left(\theta, \alpha ; E_{1}\right)$. 
Para diferenciar (3.4.16) com respeito a $\varepsilon$ definimos

$$
\begin{aligned}
\psi(\varepsilon, z, w) & =\frac{1}{f(\varepsilon, z)-w} \\
& =(r \circ[f(\varepsilon, z)-w])(\varepsilon, z, w), \quad r(x):=\frac{1}{x}
\end{aligned}
$$

e introduzimos a notação

$$
h^{\{i\}}(\varepsilon, x)=\frac{1}{i !} \frac{\partial^{i} h(\varepsilon, x)}{\partial \varepsilon^{i}} .
$$

Então, pela regra de Leibniz (3.3.14) vemos que para cada $i \geq 0$

$$
g^{\{i\}}(\varepsilon, w)=\frac{1}{2 \pi i} \int_{\mathcal{C}} z \sum_{j=0}^{i} f_{z}^{\{i-j\}}(\varepsilon, z) \psi^{\{j\}}(\varepsilon, z, w) d z
$$

$\operatorname{com} \psi^{\{0\}}(\varepsilon, z, w)=1 /(f(\varepsilon, z)-w)$ e, pela fórmula de Scott (3.3.15),

$$
\psi^{\{j\}}(\varepsilon, z, w)=\sum_{k=1}^{j} \frac{(-1)^{k}}{\left(f^{\{0\}}(\varepsilon, z)-w\right)^{k+1}} \sum_{\substack{l_{1}, \ldots, l_{k} \geq 1: \\ l_{1}+\cdots+l_{k}=j}} f^{\left\{l_{1}\right\}}(\varepsilon, z) \cdots f^{\left\{l_{k}\right\}}(\varepsilon, z)
$$

para cada $j \geq 1$. Dessas fórmulas, que valem para todo $\varepsilon$, incluindo $\varepsilon=0$, e usando a hipótese (3.4.2), estimaremos $\left|g^{\{i\}}(\varepsilon, w)\right|$.

Antes de prosseguirmos é conveniente pegarmos constantes positivas $\delta$ e $\nu>\mu$ de tal maneira que (3.4.2) dê lugar a

$$
\left|f^{\{i\}}(\varepsilon, z)\right| \leq \delta C_{i} \nu^{i}
$$

para cada $i \geq 0$ e $(\varepsilon, z) \in S\left(\theta, \alpha ; E_{1}\right) \times \overline{D_{r}\left(z_{0}\right)}$, com $C_{0}=C / \delta, C_{i}=A i !^{s} / i^{2}$ e $A$ satisfazendo (3.3.1). Isso é sempre possível: por (3.4.2) com $i=1$,

$$
C \mu \leq \delta C_{1} \nu=\delta A \nu
$$


implica

$$
\frac{C}{\delta A} \frac{\mu}{\nu} \leq 1
$$

por (3.4.2) com $i \geq 2$, juntamente com (3.4.24), temos

$$
\begin{aligned}
C i !^{s} \mu^{i} & =\frac{C i^{2}}{A \delta}\left(\frac{\mu}{\nu}\right)^{i} \delta C_{i} \nu^{i} \\
& \leq i^{2}\left(\frac{\mu}{\nu}\right)^{i-1} \delta C_{i} \nu^{i} \leq \delta C_{i} \nu^{i}
\end{aligned}
$$

contanto que $\mu / \nu \leq 1 / 4$. Então (3.4.23) vale desde que

$$
\nu \geq \mu \cdot \max \left(4, \frac{C}{\delta A}\right) .
$$

Como veremos em breve, uma escolha adequada de $\delta$ deve ser tal que

$$
\sup _{(\varepsilon, z, w) \in S\left(\theta, \alpha ; E_{1}\right) \times \mathcal{C} \times \overline{D_{m_{2}}(0)}} \frac{\delta}{\left|f^{\{0\}}(\varepsilon, z)-w\right|} \equiv c<1,
$$

com $m_{2}=m_{2}(\rho)<m_{1}(\rho)$ como no enunciado.

Iniciemos as estimativas: por (3.4.23) e pela Proposição 3.2.2 temos que para cada $i \geq 0$ e $(\varepsilon, z) \in S\left(\theta, \alpha ; E_{1}\right) \times \overline{D_{\rho}\left(z_{0}\right)}$ (lembre que $\left.\rho<r\right)$

$$
\left|f_{z}^{\{i\}}(\varepsilon, z)\right| \leq \frac{\delta C_{i} \nu^{i}}{r-\rho}
$$

Por (3.4.26) temos que para $(\varepsilon, z, w) \in S\left(\theta, \alpha ; E_{1}\right) \times \mathcal{C} \times \overline{D_{m_{2}}(0)}$ vale

$$
\left|\psi^{\{0\}}(\varepsilon, z, w)\right| \leq \frac{c}{\delta}
$$

nesse mesmo domínio vemos de (3.4.23), (3.4.26) e (3.3.4) que para cada $j \geq 1$ 


$$
\begin{aligned}
\left|\psi^{\{j\}}(\varepsilon, z, w)\right| & \leq \sum_{k=1}^{j} \frac{1}{\left|f^{\{0\}}(\varepsilon, z)-w\right|^{k+1}} \sum_{\substack{l_{1}, \ldots, l_{k} \geq 1: \\
l_{1}+\cdots+l_{k}=j}}\left|f^{\left\{l_{1}\right\}}(\varepsilon, z)\right| \cdots\left|f^{\left\{l_{k}\right\}}(\varepsilon, z)\right| \\
& \leq \frac{C_{j} \nu^{j}}{\left|f^{\{0\}}(\varepsilon, z)-w\right|} \sum_{k=1}^{j}\left(\frac{\delta}{\left|f^{\{0\}}(\varepsilon, z)-w\right|}\right)^{k} \\
& \leq \frac{c^{2}}{\delta(1-c)} C_{j} \nu^{j}
\end{aligned}
$$

As duas últimas estimativas juntamente com (3.4.27) e (3.3.3) nos conduzem a (lembrese que $\left.C_{0}=C / \delta\right)$

$$
\begin{aligned}
\sum_{j=0}^{i}\left|f_{z}^{\{i-j\}}\right|\left|\psi^{\{j\}}\right| & =\left|f_{z}^{\{i\}}\right|\left|\psi^{\{0\}}\right|+\sum_{j=1}^{i-1}\left|f_{z}^{\{i-j\}}\right|\left|\psi^{\{j\}}\right|+\left|f_{z}^{\{0\}}\right|\left|\psi^{\{i\}}\right| \\
& \leq \frac{1}{r-\rho}\left(c+\frac{c^{2}}{1-c}+\frac{C c^{2}}{\delta(1-c)}\right) C_{i} \nu^{i} \\
& =\frac{1}{r-\rho} \frac{c(\delta+C c)}{\delta(1-c)} C_{i} \nu^{i}
\end{aligned}
$$

e

$$
\begin{aligned}
\left|g^{\{i\}}(\varepsilon, w)\right| & \leq \frac{1}{2 \pi} \int_{\mathcal{C}}|z| \sum_{j=0}^{i}\left|f_{z}^{\{i-j\}}(\varepsilon, z)\right|\left|\psi^{\{j\}}(\varepsilon, z, w)\right||d z| \\
& \leq \frac{\left(\left|z_{0}\right|+\rho\right) \rho}{r-\rho} \frac{c(\delta+C c)}{\delta(1-c)} C_{i} \nu^{i} \equiv \Delta C_{i} \nu^{i}
\end{aligned}
$$

para cada $i \geq 0$ e $(\varepsilon, w) \in S\left(\theta, \alpha ; E_{1}\right) \times \overline{D_{m_{2}}(0)}$. Considerando as definições de $C_{0}$ e $C_{i}$ $(i \geq 1)$, concluímos que para cada $i \geq 0$ e $(\varepsilon, w) \in S\left(\theta, \alpha ; E_{1}\right) \times \overline{D_{m_{2}}(0)}$

$$
\left|g^{\{i\}}(\varepsilon, w)\right| \leq \widetilde{C} i !^{s} \tilde{\mu}^{i}
$$

$\operatorname{com} \widetilde{C}=\max (\Delta C / \delta, \Delta A)$ e $\tilde{\mu}=\nu$. 
Comentário 3.4.8 A equação (3.4.21) em $\varepsilon=0$ é escrita (com a notação empregada em (3.4.6) e (3.4.7))

$$
\begin{aligned}
b_{i}(w) & =\frac{1}{2 \pi i} \int_{\mathcal{C}} z a_{i}^{\prime}(z) \frac{1}{a_{0}(z)-w} d z+ \\
& +\sum_{j=1}^{i} \sum_{k=1}^{j}(-1)^{k} \sum_{\substack{l_{1}, \ldots, l_{k} \geq 1: \\
l_{1}+\cdots+l_{k}=j}} \frac{1}{2 \pi i} \int_{\mathcal{C}} z a_{i-j}^{\prime}(z) \frac{a_{l_{1}}(z) \cdots a_{l_{k}}(z)}{\left(a_{0}(z)-w\right)^{k+1}} d z \\
& =\left.\frac{z a_{i}^{\prime}(z)}{a_{0}^{\prime}(z)}\right|_{z=a_{0}^{-1}(w)}+ \\
& +\left.\sum_{j=1}^{i} \sum_{k=1}^{j}(-1)^{k} \sum_{\substack{l_{1}, \ldots, l_{k} \geq 1: \\
l_{1}+\cdots+l_{k}=j}} \frac{1}{k !} \frac{d^{k}}{d z^{k}}\left(\frac{z a_{i-j}^{\prime}(z) a_{l_{1}}(z) \cdots a_{l_{k}}(z)}{\left(a_{0}^{\prime}(z)\right)^{k+1}}\right)\right|_{z=a_{0}^{-1}(w)}
\end{aligned}
$$

pelo cálculo de resíduos (vide, por exemplo, Seção 9.1 de [26]). Note que pela condição (3.4.14) e pelo Teorema 3.4.6, $a_{0}(z)-w$ possui uma única raíz $z=a_{0}^{-1}(w)$ em $D_{r}(0)$, e definindo $c_{0}(z, w)$ pela equação

$$
a_{0}(z)-w=c_{0}(z, w)\left(z-a_{0}^{-1}(w)\right)
$$

temos $c_{0}\left(a_{0}^{-1}(w), w\right)=a_{0}^{\prime}\left(a_{0}^{-1}(w)\right)$. Isso nos dá um algoritmo para computarmos os coeficientes $b_{i}(w)$ de (3.4.7) em função dos coeficientes $a_{i}(z)$ de (3.4.6). 


\section{Capítulo 4}

\section{Estudo da Condição Inicial}

Este capítulo é destinado ao estudo da condição inicial $u_{x}^{(N)}(0, x)$ e é constituído por três seções, ao longo das quais $x$ é sempre uma variável complexa. Os resultados das duas primeiras seções são válidos para $N>2$ real; os da terceira dizem respeito ao caso em que $N$ é complexo com $|N|>>1$.

Na primeira seção mostraremos que para $x \in \mathbb{C}$ e $N>2$ real (incluindo o limite $N \rightarrow \infty) u_{x}^{(N)}(0, x)$ pertence à classe $P$ das funções de Pick (ou Herglotz). Fazendo uso da representação associada a essa classe de funções, acharemos na segunda seção o raio de univalência de $u_{x}^{(N)}(0, x)$. Na terceira seção faremos $\varepsilon=2 / N$ e estenderemos para um setor do plano complexo (setor que inclui $\mathbb{R}_{+}^{*}$ ), e faremos o estudo da somabilidade da série (formal) em potências de $\varepsilon$, série essa que (formalmente) satisfaz a mesma equação diferencial que $u_{x}^{(N)}(0, x)$.

\subsection{A Classe $P$ das Funções de Pick}

Iniciamos esta seção voltando às expressões $(2.3 .18),(2.2 .27)$ e (2.2.29). Temos

$$
\begin{aligned}
u^{(N)}(0, x) & =-\frac{1}{N} \ln \varphi_{0}^{(N)}(N x) \\
& =-\frac{1}{N} \sum_{n=1}^{\infty} \ln \left(1+\frac{x}{\frac{\alpha_{n, N / 2-1}^{2}}{\beta N^{2}}}\right)
\end{aligned}
$$


Portanto

$$
\begin{aligned}
u_{x}^{(N)}(0, x) & =\sqrt{\frac{\beta}{4 x}} \frac{i J_{N / 2}(i \sqrt{\beta x} N)}{J_{N / 2-1}(i \sqrt{\beta x} N)} \\
& =-\frac{1}{N} \sum_{n=1}^{\infty} \frac{1}{\frac{\alpha_{n, N / 2-1}^{2}}{\beta N^{2}}+x}
\end{aligned}
$$

que é a representação de uma função meromorfa com pólos de massa $1 / N$ localizados em (4.1.3). Funções meromorfas são aquelas que possuem pólos e zeros. Neste caso os zeros (não nulos) de $J_{N / 2-1}(i \sqrt{\beta x} N)$

$$
\left\{-\frac{\alpha_{n, N / 2-1}^{2}}{\beta N^{2}} \equiv-p_{n, N}, n \geq 1\right\}
$$

são os pólos de $u_{x}^{(N)}(0, x)$, e os zeros (não nulos) de $J_{N / 2}(i \sqrt{\beta x} N)$

$$
\left\{-\frac{\alpha_{n, N / 2}^{2}}{\beta N^{2}} \equiv-z_{n, N}, n \geq 1\right\}
$$

são os zeros de $u_{x}^{(N)}(0, x)$.

Observação 4.1.1 Vê-se claramente pela representação (2.2.28) que $x=0$ é um zero de ordem $N / 4-1 / 2$ de $J_{N / 2-1}(i \sqrt{\beta x} N)$, e um zero de ordem $N / 4$ de $J_{N / 2}(i \sqrt{\beta x} N)$. Desse modo $x=0$ é um zero de ordem $1 / 2$ da razão $J_{N / 2}(i \sqrt{\beta x} N) / J_{N / 2-1}(i \sqrt{\beta x} N)$, e não é um zero de $u_{x}^{(N)}(0, x)$. Note que por (2.2.28)

$$
u_{x}^{(N)}(0, x)=-\frac{\beta}{2} \prod_{n=1}^{\infty}\left(1+\frac{x}{\frac{\alpha_{n, N / 2}^{2}}{\beta N^{2}}}\right)\left(1+\frac{x}{\frac{\alpha_{n, N / 2-1}^{2}}{\beta N^{2}}}\right)^{-1}
$$

dessa e de $(4.1 .2)$ segue que $u_{x}^{(N)}(0,0)=-\beta / 2=-\beta N \sum_{n=1}^{\infty} \alpha_{n, N / 2-1}^{-2}$.

Como já exposto na demonstração da Proposição 2.2 .9 (vide página 47), para $\nu>-1$ real a função de Bessel $J_{\nu}(\xi)$ possui infinitos zeros, todos reais, sendo que seus zeros positivos $\left\{\alpha_{n, \nu}, n \geq 1\right\}$ são indexados por $n$ de tal maneira que $0<\alpha_{n, \nu}<\alpha_{n+1, \nu}$ para 
todo $n=1,2,3, \ldots$. Além disso, se $\nu>-1$ real há um entrelaçamento entre os zeros positivos de $J_{\nu}(\xi)$ e os zeros positivos de $J_{\nu+1}(\xi)$ (vide Seção 15.22 de [48]):

$$
0<\alpha_{1, \nu}<\alpha_{1, \nu+1}<\alpha_{2, \nu}<\alpha_{2, \nu+1}<\alpha_{3, \nu}<\cdots .
$$

De todos esses fatos temos que os pólos e zeros de $u_{x}^{(N)}(0, x)$ são todos reais, negativos e tais que

$$
\cdots<-p_{n+1, N}<-z_{n, N}<-p_{n, N}<\cdots<-p_{2, N}<-z_{1, N}<-p_{1, N}<0
$$

- há um entrelaçamento entre os pólos e os zeros de $u_{x}^{(N)}(0, x)$ : entre dois pólos consecutivos há um zero.

Funções que possuem a representação (4.1.2) pertencem à classe $P$ das funções de Pick (vide Apêndice A.1 para uma exposição detalhada): trata-se de funções $f(x)$ analíticas no semi-plano superior $\mathbb{H}=\{x \in \mathbb{C}: \Im m(x)>0\}$ que apresentam parte imaginária positiva para $x$ nesse domínio: $\Im m(f) \geq 0$ se $\Im m(x)>0$. A classe $P$ das funções de Pick forma um cone convexo e é fechada sob composições: para quaisquer $a, b \geq 0$ e $f_{1}, f_{2} \in P$ tem-se que

1. $a f_{1}+b f_{2} \in P$;

2. $f_{1} \circ f_{2} \in P$.

A função linear

$$
a+b x=(a+b \Re e(x))+i(b \Im m(x))
$$

com $a \in \mathbb{R}$ e $b>0$, e a função

$$
-\frac{1}{x}=\frac{-\Re e(x)+i \Im m(x)}{(\Re e(x))^{2}+(\Im m(x))^{2}},
$$

claramente pertencem à classe $P$ - ambas são mapas um-para-um e sobrejetivos de $\mathbb{H}$ em $\mathbb{H}$. Pelo Lema A.1.7, temos que $u_{x}^{(N)}(0, x)$ pertence a $P_{\left(-p_{1, N},+\infty\right)}$, uma subclasse de $P$ : por não pôr massa alguma no intervalo $\left(-p_{1, N},+\infty\right)$ do eixo real, $u_{x}^{(N)}(0, x)$ admite uma continuação analítica para o semi-plano inferior $-\mathbb{H}=\{x \in \mathbb{C}: \Im m(x)<0\}$ através desse intervalo, sendo que essa continuação é por reflexão (e assim, a função é real sobre 
$\left.\left(-p_{1, N},+\infty\right)\right)$. Além disso, $u_{x}^{(N)}(0, x)$ é uma função crescente no intervalo $\left(-p_{1, N},+\infty\right)$ (toda função pertencente a $P_{\left(c_{1}, c_{2}\right)}$ é crescente no intervalo $\left(c_{1}, c_{2}\right)$ ).

Pelas cotas superior e inferior mostradas no artigo [46] para os zeros positivos da função de Bessel $J_{\nu}(\xi)$, para $N>2$ e $n=1,2,3, \ldots$ valem

$$
\begin{aligned}
-p_{n, N}>- & \frac{1}{4 \beta}\left(1-\frac{2}{N}\right)^{2}\left(1-\frac{A_{n} 2^{1 / 3}}{(N-2)^{2 / 3}}+\frac{3}{20} \frac{A_{n}^{2} 2^{5 / 3}}{(N-2)^{4 / 3}}\right)^{2} \\
& -p_{n, N}<-\frac{1}{4 \beta}\left(1-\frac{2}{N}\right)^{2}\left(1-\frac{A_{n} 2^{1 / 3}}{(N-2)^{2 / 3}}\right)^{2}
\end{aligned}
$$

sendo que $A_{n}$ denota o $n$-ésimo zero (negativo) da função de Airy $A i(z)$. O valor de $A_{1}$ é aproximadamente $-2,33811$, de modo que no limite $N \rightarrow \infty$ vemos (pelo Teorema do Confronto, também conhecido por "Squeezing Theorem", "Pinching Theorem" e "Sandwich Theorem") que

$$
\lim _{N \rightarrow \infty}-p_{1, N}=-\frac{1}{4 \beta}
$$

Conforme $n$ cresce a ordem de $A_{n}$ também cresce, de modo que para $n$ suficientemente grande deve chegar a $O\left(N^{2 / 3}\right)$. Também por (4.1.6) e (4.1.7) devemos esperar que quando $N \rightarrow \infty$ os pólos (4.1.3) se adensem sobre um intervalo de $\mathbb{R}_{-}$- por (4.1.8), devemos esperar que sobre um intervalo de $(-\infty,-1 /(4 \beta))$.

Um passo na direção dessa questão do adensamento dos pólos é o resultado a seguir enunciado e provado, que diz respeito ao limite $N \rightarrow \infty$ de $u_{x}^{(N)}(0, x)$ - embora tal proposição já tenha sido apresentada em [7] e [41], o faremos aqui por uma questão de completeza. Na demonstração dessa proposição fazemos a extensão da demonstração feita por Watanabe [49] para o semi-plano superior $\mathbb{H}$.

\section{Proposição 4.1.2}

$$
\lim _{N \rightarrow \infty} u_{x}^{(N)}(0, x)=u_{x}^{(\infty)}(0, x)=\frac{-\beta}{1+\sqrt{1+4 \beta x}}
$$

sendo que a convergência é uniforme em qualquer subconjunto compacto do plano "slit" $\mathbb{C} \backslash(-\infty,-1 /(4 \beta)]$. 
Demonstração. Seja $\psi_{\nu}(\xi)=\xi J_{\nu}(\xi) / J_{\nu-1}(\xi)$ definida para $\nu \geq 1$ real e $\xi \in \mathbb{C}$. A relação recursiva para a função de Bessel

$$
J_{\nu-1}(\xi)+J_{\nu+1}(\xi)=\frac{2 \nu}{\xi} J_{\nu}(\xi)
$$

gera a seguinte fração continuada de Gauss (vide Capítulo XVIII de [47]):

$$
\psi_{\nu}(\xi)=\frac{2}{\nu} \frac{(\xi / 2)^{2}}{1-\frac{1}{2 \nu} \psi_{\nu+1}(\xi)}=\frac{2}{\nu} \frac{(\xi / 2)^{2}}{1-\frac{1}{\nu(\nu+1)} \frac{(\xi / 2)^{2}}{1-\frac{1}{2 \nu+2} \psi_{\nu+2}(\xi)}}
$$

Partindo da condição inicial $u^{(N)}(0, x)$ (vide (2.3.18)) e utilizando a relação de recorrência $\nu J_{\nu}(\xi)-\xi J_{\nu}^{\prime}(\xi)=\xi J_{\nu+1}(\xi)$ para as funções de Bessel, temos que

$$
\begin{aligned}
u_{x}^{(N)}(0, x) & =\frac{1}{2 N x}\left\{\frac{(N / 2-1) J_{N / 2-1}(i \sqrt{\beta x} N)-i \sqrt{\beta x} N J_{N / 2-1}^{\prime}(i \sqrt{\beta x} N)}{J_{N / 2-1}(i \sqrt{\beta x} N)}\right\} \\
& =\frac{i \sqrt{\beta x} N}{2 N x} \frac{J_{N / 2}(i \sqrt{\beta x} N)}{J_{N / 2-1}(i \sqrt{\beta x} N)}=\frac{1}{2 N x} \psi_{N / 2}(i \sqrt{\beta x} N) .
\end{aligned}
$$

Tomando $\xi=i \sqrt{\beta x} N$ e $\nu=N / 2$ em (4.1.11),

$$
\frac{1}{2 N x} \psi_{N / 2}(i \sqrt{\beta x} N)=\frac{c_{1}}{1+\frac{c_{2}}{1+\frac{c_{3}}{1+\frac{c_{4}}{1+\ddots}}}}
$$

$\operatorname{com} c_{1}=-\beta / 2$ e

$$
c_{k+1}=\frac{-1}{(\nu+k-1)(\nu+k)}\left(\frac{\xi}{2}\right)^{2}=\frac{\beta x}{\left(1+\frac{2 k-2}{N}\right)\left(1+\frac{2 k}{N}\right)}
$$

para $k=1,2,3, \ldots$, sendo que a fração continuada infinita (4.1.13) é uniformemente convergente sobre o domínio

$$
|x| \leq \frac{1}{4 \beta}\left(1+\frac{2}{N}\right)
$$


pelo Teorema de Worpitzky (vide página 42 de [47] ou Apêndice A.2):

Teorema 4.1.3 Sejam $c_{2}, c_{3}, c_{4}, \ldots$ funções de quaisquer variáveis sobre um domínio $\mathcal{D}$ no qual

$$
\left|c_{k+1}\right| \leq \frac{1}{4}, \quad k=1,2,3, \ldots
$$

Então valem as seguintes afirmações:

(a) A fração continuada (4.1.13) converge uniformemente sobre $\mathcal{D}$.

(b) Os valores da fração continuada e de seus aproximantes estão no domínio circular

$$
\left|x-\frac{4}{3}\right| \leq \frac{2}{3} .
$$

(c) A constante 1/4 é a "melhor" constante que pode ser usada em (4.1.16), e (4.1.17) é o "melhor" domínio de valores dos aproximantes.

Quando então tomamos o limite $N \rightarrow \infty$, (4.1.14) converge para $\beta x$ uniformemente sobre o domínio (4.1.15), para todo $k \geq 1$. Conseqüentemente, $u_{x}^{(N)}(0, x)$ converge sobre o mesmo domínio para uma fração continuada periódica, isto é,

$$
\lim _{N \rightarrow \infty} u_{x}^{(N)}(0, x)=\frac{-\beta / 2}{1+\frac{\beta x}{1+\frac{\beta x}{1+\ddots}}}=\frac{-\beta / 2}{\phi}=\frac{-\beta}{1+\sqrt{1+4 \beta x}}
$$

$\phi$ denota a solução $\frac{1+\sqrt{1+4 \beta x}}{2}$ da equação de ponto fixo

$$
\phi=1+\frac{\beta x}{\phi}
$$

- escolhemos a solução que é (estritamente) positiva para todo $x$ real maior que $-1 /(4 \beta)$ (e portanto não nula em $x=0$ ). Note que o limite é válido para qualquer $x$ no domínio

$$
|x| \leq \frac{1}{4 \beta}
$$


do plano complexo, sendo esse o maior domínio possível sem que se entre no corte $(-\infty,-1 /(4 \beta))$ da função limite $u_{x}^{(\infty)}(0, x)$.

Observe que $u_{x}^{(\infty)}(0, x)$ é a composição de quatro funções da classe $P$, a saber,

$$
-\frac{1}{x}, \quad \frac{1+x}{\beta}, \quad \sqrt{x}, \quad 1+4 \beta x .
$$

Pelas propriedades 1 e 2 acima mostradas, isso implica que $u_{x}^{(\infty)}(0, x)$ pertence a $P$; mais especificamente, a $P_{(-1 /(4 \beta),+\infty)}$. O que temos aqui é que $\left(u_{x}^{(N)}(0, x)\right)_{N \geq 3}$, uma seqüência de funções em $P$, é tal que $u_{x}^{(N)}(0, x)$ converge na topologia de convergência uniforme em subconjuntos compactos de $\mathbb{H}$ para $u_{x}^{(\infty)}(0, x)$ quando $N \rightarrow \infty$ (vide Apêndice A.1). Além disso, vemos que $\left(u_{x}^{(N)}(0, x)\right)_{N \geq 3}$, uma seqüência de funções em $P_{\left(-p_{1, N},+\infty\right)}$ uniformemente limitadas sobre $(-1 /(4 \beta),+\infty)$ (vide Figura 4.1), é tal que $u_{x}^{(N)}(0, x)$ converge na topologia de convergência uniforme em subconjuntos compactos de $(-1 /(4 \beta),+\infty)$ para $u_{x}^{(\infty)}(0, x)$ quando $N \rightarrow \infty$. Esses fatos implicam que as igualdades em (4.1.18) valem para $x \in \mathbb{H}$, e por reflexão através do intervalo $(-1 /(4 \beta),+\infty)$, para $x \in-\mathbb{H}$, concluindo assim a demonstração da proposição.

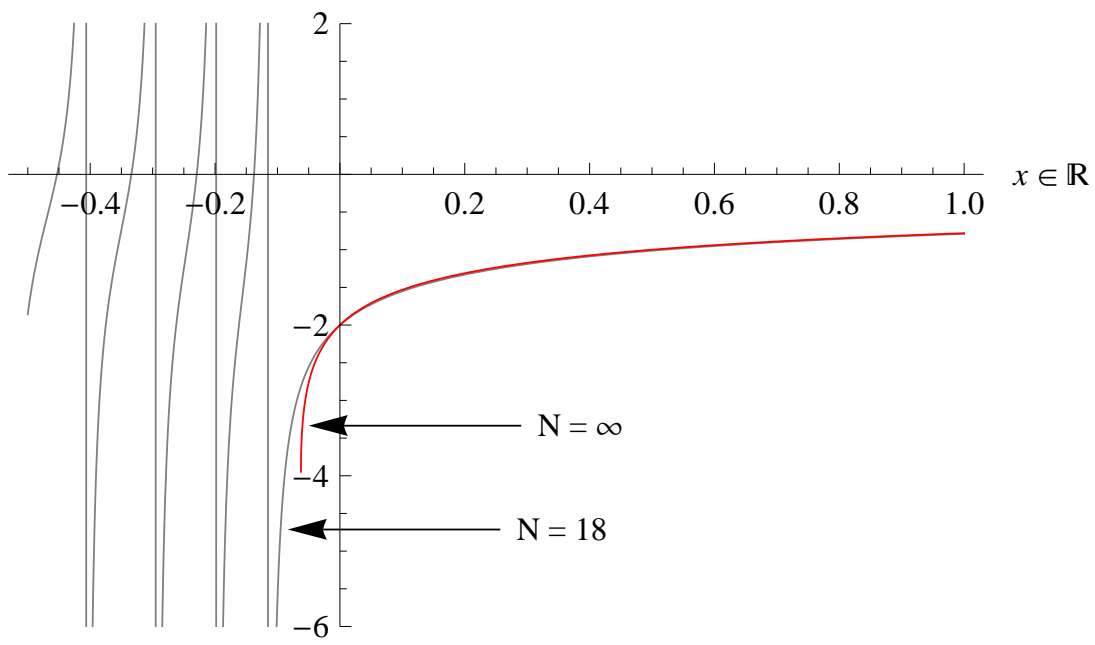

Figura 4.1: Para $x$ real e $\beta=4$ é apresentado acima o gráfico da função $u_{x}^{(N)}(0, x)$ para $N=18$ e o gráfico da função $u_{x}^{(\infty)}(0, x)$. Note que as duas funções são uniformemente limitadas sobre $(-1 /(4 \beta),+\infty)$ : existe uma constante positiva $M<\infty$ tal que $\left|u_{x}^{(N)}(0, x)\right| \leq M$ e $\left|u_{x}^{(\infty)}(0, x)\right| \leq M$ para todo $x \in(-1 /(4 \beta),+\infty)$. 
Voltando à representação (4.1.2), podemos reescrevê-la como

$$
u_{x}^{(N)}(0, x)=\int_{-\infty}^{+\infty} \frac{1}{\lambda-x} d \mu^{(N)}(\lambda)
$$

com

$$
d \mu^{(N)}(\lambda)=\frac{1}{N} \sum_{n=1}^{\infty} \delta\left(\lambda+p_{n, N}\right) d \lambda .
$$

Já para $u_{x}^{(N)}(0, x)$ (vide Teorema A.1.1 no Apêndice A.1) temos

$$
u_{x}^{(\infty)}(0, x)=\int_{-\infty}^{+\infty} \frac{1}{\lambda-x} d \mu^{(\infty)}(\lambda)
$$

com

$$
d \mu^{(\infty)}(\lambda)=\frac{\beta}{\pi} \frac{\sqrt{4 \beta(-\lambda)-1}}{4 \beta(-\lambda)} d \lambda
$$

uma medida de Borel positiva absolutamente contínua com respeito à medida de Lebesgue $d \lambda$, suportada sobre o intervalo $(-\infty,-1 /(4 \beta))$ (o corte de $u_{x}^{(\infty)}(0, x)$ ). Compare (4.1.22) com (A.1.10): fazendo $x=R+i I$, para $u_{x}^{(\infty)}(0, x)$ temos

$$
a=\lim _{I \rightarrow \infty} \frac{-\beta}{1+\sqrt{1+i 4 \beta I}} \frac{1}{i I}=0
$$

e

$$
b=\int_{-\infty}^{+\infty} \frac{\lambda}{\lambda^{2}+1} d \mu^{(\infty)}(\lambda)
$$

já tendo por conta que $a=0$, a identificação de $b$ com a integral segue de

$$
\begin{aligned}
\lim _{x \rightarrow \infty} u_{x}^{(\infty)}(0, x) & =0 \\
& =b+\lim _{x \rightarrow \infty} \int_{-\infty}^{+\infty}\left(\frac{1}{\lambda-x}-\frac{\lambda}{\lambda^{2}+1}\right) d \mu^{(\infty)}(\lambda)
\end{aligned}
$$


sendo que passamos o limite para dentro da integral pelo Teorema da Convergência de Lebesgue. A parte imaginária de $u_{x}^{(\infty)}(0, x)$ é igual a

$$
\frac{\beta \sqrt{r} \sin (\theta / 2)}{(1+\sqrt{r} \cos (\theta / 2))^{2}+(\sqrt{r} \sin (\theta / 2))^{2}},
$$

$\operatorname{com} r=\sqrt{(1+4 \beta R)^{2}+(4 \beta I)^{2}} \mathrm{e}$

$$
\theta=\arctan \left(\frac{4 \beta I}{1+4 \beta R}\right)
$$

Multiplicando (4.1.26) por $1 / \pi$ e tomando o limite $I \downarrow 0$ sobre o corte $(-\infty,-1 /(4 \beta))$, encontramos a medida (4.1.23) haja vista que

$$
\lim _{I \downarrow 0} \theta=\left\{\begin{array}{lll}
\pi & \text { se } & R<-1 /(4 \beta) \\
0 & \text { se } & R>-1 /(4 \beta)
\end{array} .\right.
$$

Dados (4.1.20)-(4.1.21) e (4.1.22)-(4.1.23), temos que no limite $N \rightarrow \infty$ a medida $d \mu^{(N)}(\lambda)$ converge fracamente para a medida $d \mu^{(\infty)}(\lambda)$ (absolutamente contínua com respeito à medida de Lebesgue $d \lambda$ ). Vemos assim que com a escala em $N$ que se tem na primeira igualdade de (4.1.1), os pólos (antes "zeros de Lee-Yang") (4.1.3) tornam-se densos sobre o intervalo $(-\infty,-1 /(4 \beta))$ de $\mathbb{R}_{-}$quando $N \rightarrow \infty$.

\subsection{Raio de Univalência}

Lembrando de (4.1.5), temos que

$$
0<p_{1, N}<p_{2, N}<\cdots<p_{n, N}<\cdots
$$

Tal ordenamento será essencial na demonstração do seguinte teorema:

Teorema 4.2.1 A condição inicial $u_{x}^{(N)}(0, x), N>2$, é univalente no disco $D_{r}(0)$ de raio $r<p_{1, N}$. 
Demonstração. Esta demonstração é dividida em três partes. Na parte I mostraremos que $h(\theta)=\left|u_{x}^{(N)}\left(0, r e^{i \theta}\right)\right|$, para $N>2,0 \leq \theta \leq \pi$ e $r<p_{1, N}$, é uma função monótona crescente; além disso, $h(2 \pi-\theta)=h(\theta)$. Na parte II mostraremos que $a(\theta)=\arg u_{x}^{(N)}\left(0, r e^{i \theta}\right)$, para $N>2$ e $r<p_{1, N}$, é tal que $\pi / 2<a(\theta)<\pi$ para $0<\theta<\pi$, e $\pi<a(\theta)<3 \pi / 2$ para $\pi<\theta<2 \pi$, sendo que $a(2 \pi-\theta)=2 \pi-a(\theta)$. Na parte III, juntando os resultados das partes anteriores e evocando o Lema 4.2.2 (vide página 121 de [38]) abaixo exposto, concluiremos o resultado enunciado no teorema.

Lema 4.2.2 Seja $\mathcal{C}$ um contorno simples fechado e $\mathcal{D}$ seu interior. Suponha que $f(x)$ é regular em $\mathcal{D}$ e contínua em $\mathcal{D} \cup \mathcal{C}$. Suponha que conforme $x$ percorre $\mathcal{C}$ na direção positiva, $p=f(x)$ percorre um contorno simples fechado $\Gamma$ uma vez. Então $\Gamma$ é percorrido positivamente, e $p=f(x)$ dá uma representação conforme de $\mathcal{D}$ em $\Delta$, o interior de $\Gamma$. [isto é, $f(x)$ é um mapa um-para-um (univalente) e sobrejetivo de $\mathcal{D}$ em $\Delta$ ]

Parte I: Pela representação (4.1.2) temos

$$
u_{x}^{(N)}\left(0, r e^{i \theta}\right)=-\frac{1}{N} \sum_{n=1}^{\infty} \frac{p_{n, N}+r \cos \theta-i r \sin \theta}{\left(p_{n, N}+r \cos \theta\right)^{2}+(r \sin \theta)^{2}} .
$$

Assim,

$$
\begin{aligned}
h^{2}(\theta) & =\frac{1}{N^{2}} \sum_{l, m \geq 1} \frac{\left(p_{l, N}+r \cos \theta\right)\left(p_{m, N}+r \cos \theta\right)+(r \sin \theta)^{2}}{\left[\left(p_{l, N}+r \cos \theta\right)^{2}+(r \sin \theta)^{2}\right]\left[\left(p_{m, N}+r \cos \theta\right)^{2}+(r \sin \theta)^{2}\right]} \\
& =\frac{1}{N^{2}} \sum_{l, m \geq 1} \frac{N_{l, m}(\theta)}{D_{l}(\theta) D_{m}(\theta)}
\end{aligned}
$$

sendo

$$
\begin{gathered}
N_{l, m}(\theta):=\left(p_{l, N}+r \cos \theta\right)\left(p_{m, N}+r \cos \theta\right)+(r \sin \theta)^{2} \\
=p_{l, N} p_{m, N}+r^{2}+\left(p_{l, N}+p_{m, N}\right) r \cos \theta \\
D_{j}(\theta):=\left(p_{j, N}+r \cos \theta\right)^{2}+(r \sin \theta)^{2} \\
=p_{j, N}^{2}+r^{2}+2 p_{j, N} r \cos \theta
\end{gathered}
$$


Note que se $r<p_{1, N}$, dado o ordenamento (4.2.1), então $N_{l, m}(\theta)>0$ para todo $\theta$ - nesse caso temos que $h(\theta)>0$ para todo $\theta$. Note também que de $\cos (2 \pi-\theta)=\cos \theta$ segue que $N_{l, m}(2 \pi-\theta)=N_{l, m}(\theta)$ e $D_{j}(2 \pi-\theta)=D_{j}(\theta)$, e portanto $h(2 \pi-\theta)=h(\theta)$.

Vamos agora mostrar que sob a condição $r<p_{1, N}$

$$
\begin{aligned}
\frac{d\left(h^{2}(\theta)\right)}{d \theta} & =\frac{1}{N^{2}} \sum_{l, m \geq 1} \frac{N_{l, m}^{\prime}(\theta) D_{l}(\theta) D_{m}(\theta)-N_{l, m}(\theta)\left[D_{l}^{\prime}(\theta) D_{m}(\theta)+D_{l}(\theta) D_{m}^{\prime}(\theta)\right]}{D_{l}^{2}(\theta) D_{m}^{2}(\theta)} \\
& =\frac{r \sin \theta}{N^{2}} \sum_{l, m \geq 1} \frac{-\left(p_{l, N}+p_{m, N}\right) D_{l} D_{m}+N_{l, m}\left[2 p_{l, N} D_{m}+2 p_{m, N} D_{l}\right]}{D_{l}^{2} D_{m}^{2}} \\
& =2 h(\theta) h^{\prime}(\theta)>0
\end{aligned}
$$

para $0<\theta<\pi$. Mais do que isso, mostraremos que sob a condição $r<p_{1, N}$ o somando mostrado na segunda linha de (4.2.6) é estritamente positivo. Como (sob a condição $\left.r<p_{1, N}\right) h(\theta)>0$ para $0 \leq \theta \leq \pi$, (4.2.6) implica que $h^{\prime}(\theta)>0$ para $0<\theta<\pi$, isto é, $h(\theta)$ é uma função monótona crescente para $0<\theta<\pi$.

Observação 4.2.3 Observe, por conta da função $\sin \theta$, que

$$
\left.\frac{d\left(h^{2}(\theta)\right)}{d \theta}\right|_{\theta=0}=\left.\frac{d\left(h^{2}(\theta)\right)}{d \theta}\right|_{\theta=\pi}=0
$$

sendo que $h_{\text {min }}=h(0)=-u_{x}^{(N)}(0, r)$ e $h_{\max }=h(\pi)=-u_{x}^{(N)}(0,-r)$ (lembre que $u_{x}^{(N)}(0, x)<0$ para $x \in\left(-p_{1, N},+\infty\right)$ - vide Figura 4.1).

Como já foi apontado, se $r<p_{1, N}$ temos que $N_{l, m}(\theta)>0$ para todo $\theta$; além disso, sabemos que $p_{j, N}>0$ para todo $j$ (vide (4.2.1)). Então, o que temos de verificar pode ser escrito na seguinte forma: sob a condição $r<p_{1, N}$, verificar que

$$
\frac{D_{l}(\theta)}{2 p_{l, N}} \frac{D_{m}(\theta)}{2 p_{m, N}}<\left(\frac{p_{l, N} p_{m, N}+r^{2}}{p_{l, N}+p_{m, N}}+r \cos \theta\right)\left[\frac{D_{l}(\theta)}{2 p_{l, N}}+\frac{D_{m}(\theta)}{2 p_{m, N}}\right]
$$

(i) Caso $l=m$ : fazendo $l=m$ em (4.2.7) e considerando a definição (4.2.5), somos conduzidos a 


$$
\left(\frac{D_{l}(\theta)}{2 p_{l, N}}\right)^{2}<2\left(\frac{D_{l}(\theta)}{2 p_{l, N}}\right)^{2}
$$

o que obviamente é verdadeiro.

(ii) Caso $l \neq m$ : note que se efetuarmos a troca de $l$ por $m$ em (4.2.7), essa expressão permanece inalterada. Assim, se mostrarmos a validade dessa para $m>l$, o caso $m<l$ fica automaticamente mostrado. Então, para $m>l$, iniciamos por observar a expressão entre parênteses em (4.2.7): lembrando de (4.2.5), temos que

$$
\frac{p_{l, N} p_{m, N}+r^{2}}{p_{l, N}+p_{m, N}}+r \cos \theta=\frac{p_{l, N} p_{m, N}+r^{2}}{p_{l, N}+p_{m, N}}-\frac{p_{l, N}^{2}+r^{2}}{2 p_{l, N}}+\frac{D_{l}(\theta)}{2 p_{l, N}}
$$

Assim, (4.2.7) fica

$$
0<\left(\frac{p_{l, N} p_{m, N}+r^{2}}{p_{l, N}+p_{m, N}}-\frac{p_{l, N}^{2}+r^{2}}{2 p_{l, N}}\right)\left[\frac{D_{l}(\theta)}{2 p_{l, N}}+\frac{D_{m}(\theta)}{2 p_{m, N}}\right]+\left(\frac{D_{l}(\theta)}{2 p_{l, N}}\right)^{2}
$$

bastando que verifiquemos que sob a condição $r<p_{1, N}$ a expressão entre parênteses é estritamente positiva: de fato,

$$
\begin{gathered}
\frac{p_{l, N} p_{m, N}+r^{2}}{p_{l, N}+p_{m, N}}-\frac{p_{l, N}^{2}+r^{2}}{2 p_{l, N}}>0 \Longleftrightarrow \frac{p_{l, N} p_{m, N}+r^{2}+p_{l, N}^{2}-p_{l, N}^{2}}{p_{l, N}+p_{m, N}}>\frac{p_{l, N}^{2}+r^{2}}{2 p_{l, N}} \\
\Longleftrightarrow \frac{p_{l, N} p_{m, N}-p_{l, N}^{2}}{p_{l, N}+p_{m, N}}>\frac{p_{l, N}^{2}+r^{2}}{2 p_{l, N}}-\frac{p_{l, N}^{2}+r^{2}}{p_{l, N}+p_{m, N}}=\frac{\left(p_{l, N}^{2}+r^{2}\right)\left(p_{m, N}-p_{l, N}\right)}{2 p_{l, N}\left(p_{l, N}+p_{m, N}\right)} \\
\Longleftrightarrow 2 p_{l, N}\left(p_{l, N} p_{m, N}-p_{l, N}^{2}\right)>\left(p_{l, N}^{2}+r^{2}\right)\left(p_{m, N}-p_{l, N}\right) \\
\Longleftrightarrow 2 p_{l, N}^{2} p_{m, N}-2 p_{l, N}^{3}>p_{l, N}^{2} p_{m, N}-p_{l, N}^{3}+r^{2}\left(p_{m, N}-p_{l, N}\right) \\
\Longleftrightarrow p_{l, N}^{2} p_{m, N}-p_{l, N}^{3}>r^{2}\left(p_{m, N}-p_{l, N}\right) \Longleftrightarrow p_{l, N}^{2}\left(p_{m, N}-p_{l, N}\right)>r^{2}\left(p_{m, N}-p_{l, N}\right)
\end{gathered}
$$


se, e somente se, $r^{2}<p_{l, N}^{2}$ para todo $l \geq 1$. Isso é verdade, pois $r<p_{1, N}$ por hipótese e há o ordenamento (4.2.1).

Por $(i)$ e $(i i)$ verificamos (4.2.6), e isso conclui a demonstração da parte I.

Parte II: Por (4.2.2) temos

$$
\begin{aligned}
& \Re e\left(u_{x}^{(N)}\left(0, r e^{i \theta}\right)\right)=-\frac{1}{N} \sum_{n=1}^{\infty} \frac{p_{n, N}+r \cos \theta}{\left(p_{n, N}+r \cos \theta\right)^{2}+(r \sin \theta)^{2}} \\
& \Im m\left(u_{x}^{(N)}\left(0, r e^{i \theta}\right)\right)=\frac{1}{N} \sum_{n=1}^{\infty} \frac{r \sin \theta}{\left(p_{n, N}+r \cos \theta\right)^{2}+(r \sin \theta)^{2}} .
\end{aligned}
$$

Sob a condição $r<p_{1, N}$, haja vista o ordenamento (4.2.1), temos que $p_{n, N}+r \cos \theta>$ 0 para todo $\theta$ e todo $n \geq 1$. Logo $\Re e\left(u_{x}^{(N)}\left(0, r e^{i \theta}\right)\right)<0$ para todo $\theta$. Quanto ao sinal de $\Im m\left(u_{x}^{(N)}\left(0, r e^{i \theta}\right)\right)$, esse é determinado pelo sinal da função $\sin \theta$ : (estritamente) positivo para $0<\theta<\pi$ (o que é esperado, dado que $u_{x}^{(N)}(0, x)$ pertence à classe $P$ ) e (estritamente) negativo para $\pi<\theta<2 \pi$. Além disso,

$$
\begin{aligned}
& \lim _{\theta \downarrow 0} \Im m\left(u_{x}^{(N)}\left(0, r e^{i \theta}\right)\right)=\lim _{\theta \uparrow \pi} \Im m\left(u_{x}^{(N)}\left(0, r e^{i \theta}\right)\right)=0^{+} \\
& \lim _{\theta \downarrow \pi} \Im m\left(u_{x}^{(N)}\left(0, r e^{i \theta}\right)\right)=\lim _{\theta \uparrow 2 \pi} \Im m\left(u_{x}^{(N)}\left(0, r e^{i \theta}\right)\right)=0^{-} .
\end{aligned}
$$

Dado esse quadro, concluímos que

$$
a(\theta)=\arg u_{x}^{(N)}\left(0, r e^{i \theta}\right)=\arctan \left(\frac{\Im m\left(u_{x}^{(N)}\left(0, r e^{i \theta}\right)\right)}{\Re e\left(u_{x}^{(N)}\left(0, r e^{i \theta}\right)\right)}\right)
$$

é tal que $\pi / 2<a(\theta)<\pi$ para $0<\theta<\pi$, e $\pi<a(\theta)<3 \pi / 2$ para $\pi<\theta<2 \pi$, sendo que $a(2 \pi-\theta)=2 \pi-a(\theta)$ (pois $\sin (2 \pi-\theta)=-\sin \theta$ e $-\tan a=\tan (2 \pi-a)) \mathrm{e}$

$$
\begin{aligned}
& \lim _{\theta \downarrow 0} a(\theta)=\lim _{\theta \uparrow \pi} a(\theta)=\pi^{-} \\
& \lim _{\theta \downarrow \pi} a(\theta)=\lim _{\theta \uparrow 2 \pi} a(\theta)=\pi^{+} .
\end{aligned}
$$


Isso fecha a parte II.

Parte III : Consideremos $u_{x}^{(N)}(0, x)$ para qualquer $N>2$ fixo. Fixemos também $r$ em um valor $r<p_{1, N}$ qualquer. Seja $\mathcal{C}=\{x \in \mathbb{C}:|x|=r\}$ a circunferência de raio $r$ no plano complexo $x$. Para mostrarmos que $u_{x}^{(N)}(0, x)$ é univalente no disco $D_{r}(0)$, em virtude do Lema 4.2.2, é necessário que garantamos que conforme $x$ percorre $\mathcal{C}$ na direção positiva, $p=u_{x}^{(N)}(0, x)$ [não confundir $p$ com $p_{n, N}$ ] percorre um contorno simples fechado $\Gamma$ uma vez. Para tanto, usaremos o que foi aprendido nas partes I e II.

Seja $p=u_{x}^{(N)}(0, x)$. Fazendo $x=r e^{i \theta}$, temos que $u_{x}^{(N)}\left(0, r e^{i \theta}\right)=h(\theta) e^{i a(\theta)}$ é o ponto $p=s+i q$ escrito em coordenadas polares (parametrizado por $\theta \in[0,2 \pi)$ ). Partamos de $\theta=0$ : sabemos que $h(0)=h_{\min }$ e $a(0)=\pi$, o que corresponde ao ponto $(s, q)=\left(-h_{\min }, 0\right)$ do plano $p$. Quando fazemos $\theta$ variar de modo crescente no intervalo $(0, \pi), h(\theta)$ cresce monotonicamente e $a(\theta)$ pertence ao segundo quadrante do plano $p$. Note que para $0<\theta_{1}<\theta_{2}<\pi$ temos

$$
\left|p_{1}-p_{2}\right|=\left|u_{x}^{(N)}\left(0, r e^{i \theta_{1}}\right)-u_{x}^{(N)}\left(0, r e^{i \theta_{2}}\right)\right| \geq h\left(\theta_{2}\right)-h\left(\theta_{1}\right)>0
$$

por conta de $h(\theta)$ ser uma função monótona crescente em $(0, \pi)$; (4.2.17) mostra que pontos distintos $x_{1}=r e^{i \theta_{1}}$ e $x_{2}=r e^{i \theta_{2}}\left(0<\theta_{1}<\theta_{2}<\pi\right)$ são levados pelo mapa $u_{x}^{(N)}(0, x)$ a pontos distintos $p_{1}=h\left(\theta_{1}\right) e^{i a\left(\theta_{1}\right)}$ e $p_{2}=h\left(\theta_{2}\right) e^{i a\left(\theta_{2}\right)}$. Chegando a $\theta=\pi$, sabemos que $h(\pi)=h_{\max }$ e $a(\pi)=\pi$, o que corresponde ao ponto $(s, q)=\left(-h_{\max }, 0\right)$ do plano $p$. Quando fazemos $\theta$ variar de modo crescente no intervalo $(\pi, 2 \pi), h(\theta)$ decresce monotonicamente e $a(\theta)$ pertence ao terceiro quadrante do plano $p$. Note que para $\pi<\theta_{3}<\theta_{4}<2 \pi$ temos

$$
\left|p_{3}-p_{4}\right|=\left|u_{x}^{(N)}\left(0, r e^{i \theta_{3}}\right)-u_{x}^{(N)}\left(0, r e^{i \theta_{4}}\right)\right| \geq h\left(\theta_{3}\right)-h\left(\theta_{4}\right)>0
$$

por conta de $h(\theta)$ ser uma função monótona decrescente em $(\pi, 2 \pi)$; (4.2.18) mostra que pontos distintos $x_{3}=r e^{i \theta_{3}}$ e $x_{4}=r e^{i \theta_{4}}\left(\pi<\theta_{3}<\theta_{4}<2 \pi\right)$ são levados pelo mapa $u_{x}^{(N)}(0, x)$ a pontos distintos $p_{3}=h\left(\theta_{3}\right) e^{i a\left(\theta_{3}\right)}$ e $p_{4}=h\left(\theta_{4}\right) e^{i a\left(\theta_{4}\right)}$. Por fim, chegamos a $\theta=2 \pi$ que é equivalente a retornar para $\theta=0$.

De toda essa descrição concluímos que conforme $x$ percorre $\mathcal{C}$ na direção positiva, $p=u_{x}^{(N)}(0, x)$ percorre um contorno simples fechado $\Gamma$ uma vez. Se introduzirmos as 
notações $\mathcal{C}^{+}=\{x \in \mathbb{H}:|x|=r\}$ e $\mathcal{C}^{-}=\{x \in-\mathbb{H}:|x|=r\}$ (semi-circunferência superior e inferior de raio $r$, respectivamente), o que vimos é que

$$
\Gamma^{+}=\left\{p=u_{x}^{(N)}(0, x) \in \mathbb{C}: x \in \mathcal{C}^{+}\right\} \subset \mathbb{H}
$$

e que

$$
\Gamma^{-}=\left\{p=u_{x}^{(N)}(0, x) \in \mathbb{C}: x \in \mathcal{C}^{-}\right\} \subset-\mathbb{H}
$$

Além disso, tendo em vista que $h(2 \pi-\theta)=h(\theta)$ e $a(2 \pi-\theta)=2 \pi-a(\theta)$, a curva $\Gamma$ é simétrica com respeito ao eixo $s$ (vide exemplo mostrado na Figura 4.2). Isso conclui a demonstração do teorema.

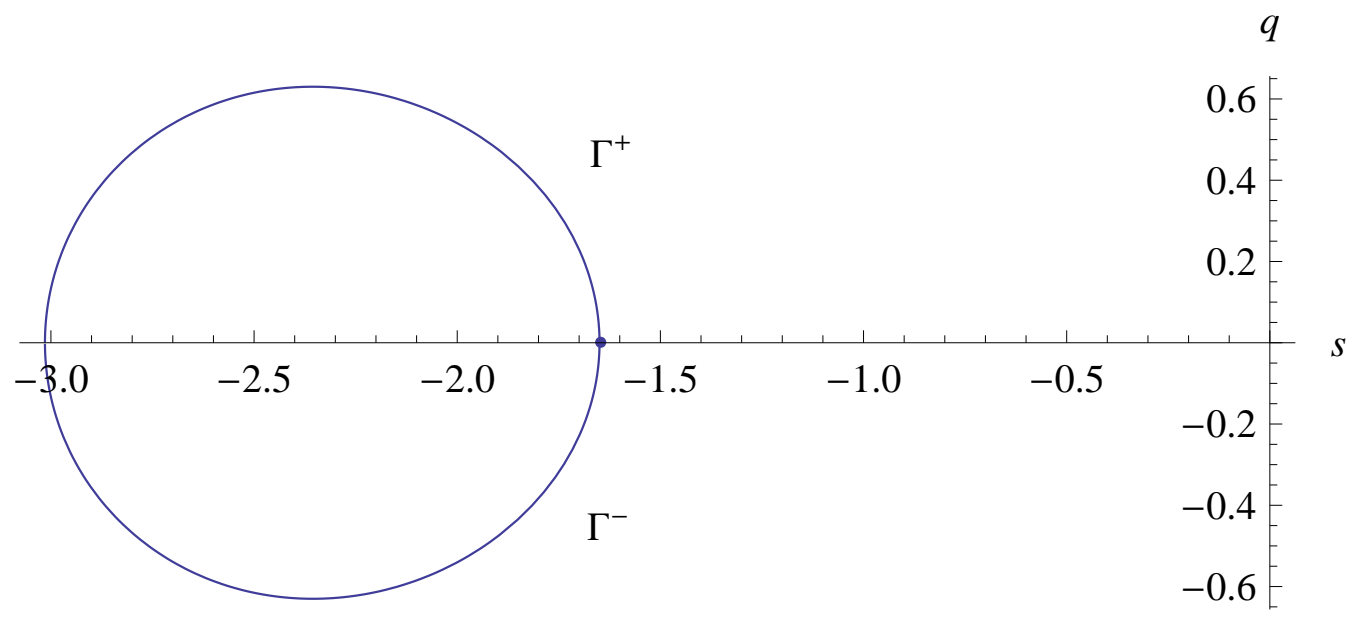

Figura 4.2: Curva $\Gamma$ obtida com as escolhas $\beta=4, N=18$ e $r=\frac{1}{4 \beta}\left(1+\frac{2}{N}\right)$.

\subsection{1-Somabilidade na Direção $\theta=0$}

Começaremos esta seção deduzindo a equação diferencial ordinária satisfeita pela função $\phi(\varepsilon, x)=u_{x}(\varepsilon, 0, x)$, e então: 
(a) mostraremos que a EDO possui uma solução analítica $\phi(\varepsilon, x)$ no domínio $S(0, \gamma ; E) \times$ $D_{\kappa}(0)$, com $\gamma$ e $\kappa$ constantes apropriadamente escolhidas, e que $\phi(\varepsilon, x)$ converge, quando $\varepsilon \rightarrow 0$ em $S(0, \gamma ; E)$, para $\phi(0, x)$, que é a solução da EDO quando $\varepsilon=0$;

(b) mostraremos que a série formal

$$
\widehat{\phi}(\varepsilon, x)=\sum_{i=0}^{\infty} f_{i}(x) \varepsilon^{i}
$$

é 1-Gevrey, para todo $x \in \overline{D_{\sigma}(0)}$ fixo, $\sigma<\kappa$;

(c) mostraremos que para constantes positivas $C$ e $\mu$

$$
\left|\frac{1}{i !} \frac{\partial^{i} \phi(\varepsilon, x)}{\partial \varepsilon^{i}}\right| \leq C i ! \mu^{i}
$$

vale para $i=0,1,2, \ldots$ e $(\varepsilon, x) \in S(0, \gamma ; E) \times \overline{D_{\sigma_{1}}(0)}, \sigma_{1}<\sigma$.

Ao final da seção, por $(a)-(c)$, concluiremos que a solução formal $\widehat{\phi}(\varepsilon, x)$ é 1-somável na direção $\theta=0$.

\subsubsection{A EDO Satisfeita por $u(\varepsilon, 0, x)=u^{(N)}(0, x)$}

Partindo de (2.3.18) e utilizando as definições $\nu:=N / 2, k:=\sqrt{\beta} N, r:=i \sqrt{x}$ e $s:=k r$, escrevemos

$$
u^{(N)}(0, x)=-\frac{1}{N} \ln \left[\frac{2^{\nu-1} \Gamma(\nu)}{s^{\nu-1}} J_{\nu-1}(s)\right] .
$$

Como bem se sabe, a função de Bessel de ordem $\nu-1\left(J_{\nu-1}(s)\right)$ satisfaz a equação diferencial ordinária

$$
\left(s J_{\nu-1}^{\prime}(s)\right)^{\prime}+\left(s-\frac{(\nu-1)^{2}}{s}\right) J_{\nu-1}(s)=0
$$

e portanto a função

$$
\varphi_{\nu}(r)=\frac{2^{\nu-1} \Gamma(\nu)}{(k r)^{\nu-1}} J_{\nu-1}(k r)
$$


satisfaz

$$
\varphi_{\nu}^{\prime \prime}(r)+\frac{2 \nu-1}{r} \varphi_{\nu}^{\prime}(r)+k^{2} \varphi_{\nu}(r)=0
$$

Por fim, a função

$$
u^{(N)}(x)=-\frac{1}{N} \ln \varphi_{N / 2}(i \sqrt{x})
$$

satisfaz (lembrando que $\nu=N / 2$ e $k^{2}=\beta N^{2}$ )

$$
\frac{2}{N} x u^{\prime \prime}(x)+u^{\prime}(x)-2 x\left(u^{\prime}(x)\right)^{2}+\frac{\beta}{2}=0 .
$$

Usando a definição $\varepsilon:=2 / N$, fica assim mostrado que $\phi(\varepsilon, x)=u_{x}(\varepsilon, 0, x)$ satisfaz

$$
\varepsilon x \phi^{\prime}(x)+\phi(x)-2 x(\phi(x))^{2}+\frac{\beta}{2}=0 .
$$

Comentário 4.3.1 A equação de Riccati

$$
\left(\sigma_{\nu}(\xi)\right)^{\prime}+\left[\frac{2 \nu+1}{\xi}\right] \sigma_{\nu}(\xi)-\left(\sigma_{\nu}(\xi)\right)^{2}-1=0
$$

está relacionada à equação (4.3.7) pelas mudanças $\xi=i \sqrt{\beta x} N, \nu=N / 2-1 e$

$$
\frac{-\beta(\nu+1)}{\xi} \sigma_{\nu}(\xi)=u_{x}^{(N)}(0, x) .
$$

Comentário 4.3.2 A equação (4.3.7) com $N=\infty$ (ou, equivalentemente, (4.3.8) com $\varepsilon=0)$ possui duas soluções, mas somente uma delas é regular em $x=0$, a saber,

$$
\frac{-\beta}{1+\sqrt{1+4 \beta x}} \text {. }
$$

Como já aprendemos na Proposição 4.1.2, é essa solução que pertence à classe P. 


\subsubsection{Série de Potências na Variável $x$}

Lema 4.3.3 Existe $\gamma$, E e $\kappa$ tais que (4.3.8) tem uma única solução $\phi(\varepsilon, x)$ analítica no domínio $S(0, \gamma ; E) \times D_{\kappa}(0)$. Essa solução $\phi(\varepsilon, x)$ converge, quando $\varepsilon \rightarrow 0$ no setor $S(0, \gamma ; E)$, para (4.3.11), que é a solução de (4.3.8) (regular em $x=0$ ) quando $\varepsilon=0$.

Demonstração. Substituindo a expansão

$$
\phi(\varepsilon, x)=\sum_{n=0}^{\infty} a_{n}(\varepsilon) x^{n}
$$

em (4.3.8), somos levados a

$$
\begin{gathered}
a_{0}(\varepsilon)+\frac{\beta}{2}=0 \\
(\varepsilon n+1) a_{n}(\varepsilon)-2 \sum_{m=0}^{n-1} a_{m}(\varepsilon) a_{n-1-m}(\varepsilon)=0 \quad(n \geq 1),
\end{gathered}
$$

de tal maneira que para cada $n$ fixo, $a_{n}(\varepsilon)$ é unicamente determinado em termo dos coeficientes anteriores.

Comecemos por notar que $(\varepsilon n+1)^{-1}$ possui pólos em $\{-1 / n, n \geq 1\}$ no plano complexo $\varepsilon$, e que para todo $n \geq 1$ e $\varepsilon \in S(0, \gamma ; \infty)$, com $\gamma<2 \pi$, vale

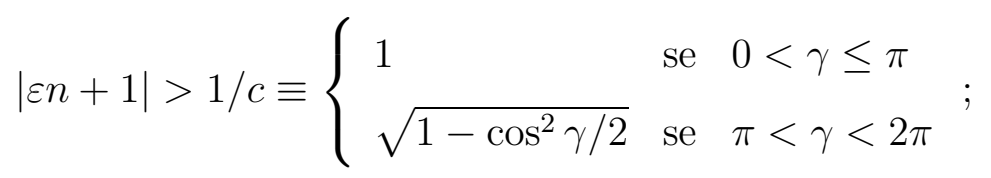

em particular, (4.3.15) vale para $\varepsilon \in S(0, \gamma ; E)$, com $E>0$ qualquer. Note que a condição $\gamma<2 \pi$ é imposta a fim de se evitar os pólos acima mencionados. Antes de prosseguirmos, mostremos (4.3.15): escrevamos $\varepsilon=\rho e^{i \theta}-$ para $\varepsilon \in S(0, \gamma ; E)$ temos então que $\rho>0$ e $-\gamma / 2<\theta<\gamma / 2$. Claramente $\cos \theta>\cos \gamma / 2$, de modo que

$$
\begin{aligned}
|\varepsilon n+1|^{2} & =(\rho n)^{2}+2(\rho n) \cos \theta+1 \\
& >(\rho n)^{2}+2(\rho n) \cos \gamma / 2+1=: h(\rho n)
\end{aligned}
$$


Assim sendo,

(i) se $0<\gamma \leq \pi, \cos \gamma / 2 \geq 0$, e portanto $h(\rho n) \geq(\rho n)^{2}+1>1$;

(ii) se $\pi<\gamma<2 \pi, \cos \gamma / 2<0$; estudando $h(\rho n)$, vê-se facilmente que seu mínimo é atingido em $\rho_{n}^{*}=(-\cos \gamma / 2) / n: h(\rho n) \geq h\left(\rho_{n}^{*} n\right)=1-\cos ^{2} \gamma / 2$.

A seguir mostraremos por indução que para cada $n \geq 0$ vale

$$
\sup _{\varepsilon \in S(0, \gamma ; E)}\left|a_{n}(\varepsilon)\right| \equiv y_{n} \leq \frac{\beta / 2}{\kappa^{n}},
$$

sendo $\kappa$ uma constante tal que

$$
0<\kappa \leq \frac{A}{4 c \beta}
$$

com $c$ a constante definida em (4.3.15) e $A$ uma constante que satisfaz (3.3.1): de (4.3.13) temos imediatamente que $y_{0}=\beta / 2$. Agora, fazendo $y_{0}=\alpha C_{0}$ e supondo que para $l=1,2, \ldots, n-1(\operatorname{com} n \geq 2)$

$$
y_{l} \leq \alpha C_{l} \kappa^{-l}
$$

com $C_{0}=A$ e $C_{l}=A / l^{2}$ para $l \geq 1$, segue de (4.3.14), (4.3.15) e (3.3.3) que

$$
\begin{aligned}
y_{n} & \leq 2 c \sum_{m=0}^{n-1} y_{m} y_{n-1-m} \\
& \leq 2 c \alpha^{2} \kappa^{-(n-1)} \sum_{m=0}^{n-1} C_{m} C_{n-1-m} \\
& \leq 2 c \alpha^{2} C_{n-1} \kappa^{-(n-1)} \leq \alpha C_{n} \kappa^{-n},
\end{aligned}
$$

sendo que a última desigualdade é válida contanto que (4.3.18) seja satisfeita - note que $\alpha=\beta /(2 A)$ e $C_{n} / C_{n-1}=(1-1 / n)^{2} \geq 1 / 4$ para todo $n \geq 2$. Para fecharmos a indução resta-nos verificar que $y_{1} \leq \alpha C_{1} \kappa^{-1}$ : por (4.3.14) (para $\left.n=1\right)$ temos 


$$
y_{1} \leq 2 c y_{0}^{2}=2 c \alpha^{2} C_{0}^{2} \leq \alpha C_{1} \kappa^{-1}
$$

contanto que $\kappa \leq 1 /(c \beta)$, e portanto válida se (4.3.18) - leve em conta que $C_{0}=C_{1}=A$ e $\alpha=\beta /(2 A)$. Chegamos assim a (4.3.17), dado que $C_{n} \leq A$ para todo $n \geq 1 \mathrm{e}$ $\alpha A=\beta / 2$.

Por (4.3.17) temos que (4.3.12) é majorada por uma série convergente para todo $\varepsilon \in S(0, \gamma ; E)$ e todo $x \in D_{\kappa}(0)$ :

$$
\phi(\varepsilon, x)=\sum_{n=0}^{\infty} a_{n}(\varepsilon) x^{n}<<\sum_{n=0}^{\infty} \frac{\beta / 2}{\kappa^{n}} x^{n}=\frac{\beta \kappa}{2(\kappa-x)} .
$$

Segue pelo Teste M de Weierstrass que a solução em série de potências (4.3.12) da equação (4.3.8) converge uniformemente no domínio $S^{\prime} \times \overline{D_{\sigma}(0)}$, com $S^{\prime}$ qualquer subsetor próprio de $S(0, \gamma ; E)$ e $\sigma<\kappa$. Portanto, definindo

$$
\phi_{m}(\varepsilon, x):=\sum_{n=0}^{m} a_{n}(\varepsilon) x^{n}
$$

temos que $\left(\phi_{m}(\varepsilon, x)\right)_{m \geq 0}$, uma seqüência de funções analíticas em $S(0, \gamma ; E) \times D_{\kappa}(0)$ (note que por (4.3.13) e (4.3.14) $\left(a_{n}(\varepsilon) x^{n}\right)_{n \geq 0}$ é uma seqüência de funções analíticas em $\left.S(0, \gamma ; E) \times D_{\kappa}(0)\right)$, é tal que $\phi_{m}(\varepsilon, x)$ converge uniformemente para $\phi(\varepsilon, x)$ sobre todo compacto $S^{\prime} \times \overline{D_{\sigma}(0)}$ de $S(0, \gamma ; E) \times D_{\kappa}(0)$ quando $m \rightarrow \infty$. Conclui-se pelo Teorema 3.3.6 que $\phi(\varepsilon, x)$ é uma função analítica em $S(0, \gamma ; E) \times D_{\kappa}(0)$.

Por fim, da convergência uniforme de (4.3.12), conclui-se que para $x \in D_{\kappa}(0)$ fixo, a solução $\phi(\varepsilon, x)$ tende a

$$
\phi(0, x)=\sum_{n=0}^{\infty} a_{n}(0) x^{n}=\frac{-\beta}{1+\sqrt{1+4 \beta x}}
$$

quando $\varepsilon \rightarrow 0$ em $S(0, \gamma ; E)$. 
Comentário 4.3.4 A representação produto canônica (2.2.28) para $J_{\nu}(\xi)$ é válida para todo $\nu \in \mathbb{C} \backslash\{-1,-2,-3, \ldots\}$ (vide Seções 15.4-15.54 de [48])1. Assim sendo, a representação (4.1.2) é válida para $N / 2 \in \mathbb{C} \backslash\{0,-1,-2, \ldots\}$ e temos que em uma vizinhança de $x=0$

$$
u_{x}^{(N)}(0, x)=\sum_{n=0}^{\infty} a_{n}^{(N)} x^{n}
$$

com

$$
a_{n}^{(N)}=\frac{\left(-\beta N^{2}\right)^{n+1}}{N} \sum_{j=1}^{\infty} \alpha_{j, N / 2-1}^{-2(n+1)} \quad(n \geq 0)
$$

Dado que $\varepsilon=2 / N, \phi(\varepsilon, x)=u_{x}^{(N)}(0, x)$ e (4.3.12), temos que $a_{n}(\varepsilon)=a_{n}^{(N)}$. Assim, (4.3.13)-(4.3.14) gera os coeficientes $b_{n}^{(N)}:=\sum_{j=1}^{\infty} \alpha_{j, N / 2-1}^{-2(n+1)}$ recursivamente:

$$
b_{n}^{(N)}=(N / 2+n)^{-1} \sum_{k=0}^{n-1} b_{k}^{(N)} b_{n-1-k}^{(N)} .
$$

Há alguns meses, quando esta subseção já estava escrita, tivemos conhecimento da referência [24]. Nessa, a relação (4.3.26) escrita em termos de $\nu=N / 2-1$ possui um papel chave: Hall a emprega para computar os zeros de menores índices $n$ de $J_{\nu}(\xi)$. Para obtê-la, procede da seguinte maneira: mostra que $\sigma_{\nu}(\xi) \equiv J_{\nu+1}(\xi) / J_{\nu}(\xi)$ satisfaz a equação de Riccati (4.3.9); substitui a representação $\sigma_{\nu}(z)=\sum_{n=1}^{\infty} 2 z\left(\alpha_{n, \nu}^{2}-z^{2}\right)^{-1}$ (compare com (4.1.2)) na equação, define $t=z^{2} / 4$ e considera a expansão de Taylor em uma vizinhança de $t=0$; por fim, coleta os coeficientes de mesma potência de $t$.

\subsubsection{Série de Potências na Variável $\varepsilon=2 / N$}

Lema 4.3.5 Suponha que a série formal (4.3.1) satisfaça a equação (4.3.8). Então, os coeficientes $\left(f_{i}(x)\right)_{i \geq 0}$ são funções analíticas de $x$ no disco aberto $D_{1 /(4 \beta)}(0)$. Além disso, existem constantes positivas $C$ e $\mu$ tais que

\footnotetext{
${ }^{1}$ Quando $\nu>-1$ real vimos que os zeros de $J_{\nu}(\xi)$ são todos reais e esses são indexados por $n$ de tal maneira que $0<\alpha_{n, \nu}<\alpha_{n+1, \nu}$ para todo $n=1,2,3, \ldots$ Todavia, quando $\nu$ é complexo os zeros tornam-se complexos, e como é dito em [24] "não há garantia alguma de que $\alpha_{n-1, \nu}$ e $\alpha_{n+1, \nu}$ são os zeros mais próximos de $\alpha_{n, \nu}$, ou que $\alpha_{1, \nu}$ é o zero mais próximo da origem."
} 


$$
\left|f_{i}(x)\right| \leq C i ! \mu^{i}
$$

vale para cada $i \geq 0$ e $x \in \overline{D_{\sigma}(0)}$, com $\sigma<\kappa<1 /(4 \beta)$. Em outras palavras, a série formal $\widehat{\phi}(\varepsilon, x)$ é 1-Gevrey, para todo $x \in \overline{D_{\sigma}(0)}$ fixo.

Demonstração. Substituindo a série formal (4.3.1) em (4.3.8), somos levados a

$$
\begin{gathered}
f_{0}(x)-2 x\left(f_{0}(x)\right)^{2}+\frac{\beta}{2}=0 \\
x f_{i-1}^{\prime}(x)+f_{i}(x)-2 x \sum_{j=0}^{i} f_{j}(x) f_{i-j}(x)=0 \quad(i \geq 1) .
\end{gathered}
$$

Resolvendo a primeira equação e escolhendo a solução que é regular em $x=0$ (vide Comentário 4.3.2), temos

$$
f_{0}(x)=\frac{-\beta}{1+\sqrt{1+4 \beta x}}
$$

Da segunda equação segue que

$$
\begin{gathered}
f_{1}(x)=\frac{1}{1-4 x f_{0}(x)}\left\{-x f_{0}^{\prime}(x)\right\} \\
f_{i}(x)=\frac{1}{1-4 x f_{0}(x)}\left\{-x f_{i-1}^{\prime}(x)+2 x \sum_{j=1}^{i-1} f_{j}(x) f_{i-j}(x)\right\} \quad(i \geq 2),
\end{gathered}
$$

e tal relação determina $f_{i}(x)$ em termo dos coeficientes anteriores de maneira única. Note que

$$
1-4 x f_{0}(x)=\sqrt{1+4 \beta x}
$$

é analítica e não se anula em $D_{1 /(4 \beta)}(0)$, e portanto $f_{i}(x)$ é analítica nesse domínio. Pegando $\kappa<1 /(4 \beta)$, notemos que 


$$
\sqrt{1-4 \beta \kappa} \equiv 1 / c_{1} \leq|\sqrt{1+4 \beta x}| \leq \sqrt{1+4 \beta \kappa} \equiv c_{2}
$$

para todo $x \in \overline{D_{\kappa}(0)}$ e, conseqüentemente,

$$
\sup _{x \in \overline{D_{\kappa}(0)}}\left|f_{0}(x)\right|=\sup _{x \in \overline{D_{\kappa}(0)}}\left|\frac{-\beta}{1+\sqrt{1+4 \beta x}}\right|=\frac{\beta}{\min \left(1-1 / c_{1}, c_{2}-1\right)} \equiv c_{3} .
$$

Agora, para obtermos uma estimativa para a taxa de crescimento de $\left|f_{i}(x)\right|$, usamos as normas de Nagumo:

$$
\|f\|_{j}=\sup _{x \in D_{\kappa}(0)}\left(d_{\kappa}(x)\right)^{j}|f(x)|, \quad \operatorname{com} \quad d_{\kappa}(x)=\kappa-|x|
$$

Assumamos que para $l=1,2, \ldots, i-1(\operatorname{com} i \geq 2)$

$$
\left\|f_{l}\right\|_{l-1} \leq \delta C_{l} \nu^{l}
$$

com $C_{l}=A l !^{\lambda} / l^{2}, \lambda \geq 0$ uma constante a ser determinada e $A$ satisfazendo (3.3.1). Então, por (4.3.32), (4.3.34), (3.3.3) e das propriedades das normas de Nagumo, segue que

$$
\begin{aligned}
\left\|f_{i}\right\|_{i-1} & \leq c_{1}\left\{\|x\|_{0}\left\|f_{i-1}^{\prime}\right\|_{i-1}+2\|x\|_{0} \sum_{j=1}^{i-1}\left\|f_{j}\right\|_{j-1}\left\|f_{i-j}\right\|_{i-j}\right\} \\
& \leq c_{1}\left\{\kappa e(i-1)\left\|f_{i-1}\right\|_{i-2}+2 \kappa^{2} \sum_{j=1}^{i-1}\left\|f_{j}\right\|_{j-1}\left\|f_{i-j}\right\|_{i-1-j}\right\} \\
& \leq c_{1}\left\{\kappa e(i-1) \delta C_{i-1} \nu^{i-1}+2 \kappa^{2} \delta^{2} C_{i} \nu^{i}\right\} \\
& \leq \delta C_{i} \nu^{i}
\end{aligned}
$$

sendo que a última desigualdade vale contanto que 


$$
c_{1}\left\{\frac{\kappa e}{\nu} \frac{i-1}{i^{\lambda}}\left(\frac{i}{i-1}\right)^{2}+2 \kappa^{2} \delta\right\} \leq 1
$$

para todo $i \geq 2$, e assim devemos ter $\lambda \geq 1, \delta$ suficientemente pequeno (em razão do segundo termo) e $\nu>\kappa$ grande o suficiente. Dessa maneira, fixamos $\lambda=1$. Com relação a $\delta$ e $\nu$, devemos escolher tais constantes de tal maneira que $\left\|f_{1}\right\|_{0} \leq \delta C_{1} \nu$; por (4.3.31) e (4.3.35) vemos que

$$
\begin{aligned}
\left\|f_{1}\right\|_{0} & =\sup _{x \in D_{\kappa}(0)}\left|\frac{1}{1-4 x f_{0}(x)}\left\{-x f_{0}^{\prime}(x)\right\}\right| \\
& =\sup _{x \in D_{\kappa}(0)}\left|\frac{1}{1-4 x f_{0}(x)}\left\{\frac{-2 x}{\sqrt{1+4 \beta x}}\left(\frac{\beta}{1+\sqrt{1+4 \beta x}}\right)^{2}\right\}\right| \\
& \leq c_{1}\left\{2 \kappa c_{1} c_{3}^{2}\right\} \leq \delta C_{1} \nu
\end{aligned}
$$

é válida para algum $\delta$ pequeno e $\nu$ grande. Escolhendo $\delta$ e $\nu$ dentro dessas condições, conclui-se que

$$
\sup _{x \in D_{\kappa}(0)}\left(d_{\kappa}(x)\right)^{i-1}\left|f_{i}(x)\right| \equiv\left\|f_{i}\right\|_{i-1} \leq \delta \frac{A i !}{i^{2}} \nu^{i} \quad \forall i \geq 1
$$

Por fim, pelas propriedades 4 e 5 das normas de Nagumo temos que para cada $i \geq 1$ e todo $x \in \overline{D_{\sigma}(0)}$, com $\sigma<\kappa$, vale

$$
\left|f_{i}(x)\right| \leq \frac{\kappa}{(\kappa-\sigma)^{i}}\left\|f_{i}\right\|_{i-1} \leq C i ! \mu^{i}
$$

com $C=\kappa \delta A$ e $\mu=\nu /(\kappa-\sigma)$. A fim de se incluir o caso $i=0$, basta que escolhamos $C=\max \left(c_{3}, \kappa \delta A\right)$, tendo em vista (4.3.35). Assim, o lema está provado. 


\subsubsection{Série Assintótica}

Lema 4.3.6 Para o setor $S(0, \gamma ; E)$ e o disco fechado $\overline{D_{\sigma_{1}}(0)}, \sigma_{1}<\kappa /(1+2 c \beta \kappa)$, com $\gamma$, E e $\kappa$ como no Lema 4.3.3 e c definida em (4.3.15), existem constantes positivas $C$ e $\mu$ tais que

$$
\left|\frac{1}{i !} \frac{\partial^{i} \phi(\varepsilon, x)}{\partial \varepsilon^{i}}\right| \leq C i ! \mu^{i}
$$

vale para cada $i \geq 0 e(\varepsilon, x) \in S(0, \gamma ; E) \times \overline{D_{\sigma_{1}}(0)}$.

Demonstração. Diferenciando (4.3.8) $i$ vezes com respeito a $\varepsilon$ e usando as notações

$$
\phi_{i}(\varepsilon, x)=\frac{1}{i !} \frac{\partial^{i} \phi(\varepsilon, x)}{\partial \varepsilon^{i}} \quad \phi_{i}^{\prime}(\varepsilon, x)=\frac{\partial \phi_{i}(\varepsilon, x)}{\partial x}
$$

vemos que para cada $i \geq 1$

$$
\varepsilon x \phi_{i}^{\prime}(\varepsilon, x)+x \phi_{i-1}^{\prime}(\varepsilon, x)+\phi_{i}(\varepsilon, x)-2 x \sum_{j=0}^{i} \phi_{j}(\varepsilon, x) \phi_{i-j}(\varepsilon, x)=0 .
$$

Essa e (4.3.8) (caso $i=0$ ) podem ser reescritas como

$$
\varepsilon x \phi_{i}^{\prime}(\varepsilon, x)+A^{[i]}(\varepsilon, x) \phi_{i}(\varepsilon, x)=g_{i}(\varepsilon, x),
$$

sendo

$$
\begin{gathered}
A^{[i]}(\varepsilon, x)=1-\Upsilon^{[i]} x \phi_{0}(\varepsilon, x) \quad \operatorname{com} \quad \Upsilon^{[0]}=2 \quad \text { e } \quad \Upsilon^{[i]}=4 \forall i \geq 1, \\
g_{0}(\varepsilon, x)=-\frac{\beta}{2}, \\
g_{1}(\varepsilon, x)=-x \phi_{0}^{\prime}(\varepsilon, x)
\end{gathered}
$$

e 


$$
g_{i}(\varepsilon, x)=-x \phi_{i-1}^{\prime}(\varepsilon, x)+2 x \sum_{j=1}^{i-1} \phi_{j}(\varepsilon, x) \phi_{i-j}(\varepsilon, x) \quad(i \geq 2)
$$

é um termo que depende somente das derivadas com respeito a $\varepsilon$ de ordem inferior a $i$. A equação linear (4.3.46) é uma perturbação singular da equação com $\varepsilon=0$, e possui uma singularidade regular. Para lidarmos com essa equação faremos uso do seguinte resultado auxiliar de Balser-Kostov [3]. Temporariamente sumiremos com os índices $i$.

Seja

$$
A(\varepsilon, x)-A_{0}(\varepsilon)=\sum_{n=1}^{\infty} A_{n}(\varepsilon) x^{n}=-B(\varepsilon, x)
$$

e consideremos a seqüência de soluções $\left(\psi_{k}(\varepsilon, x)\right)_{k \geq 0}$ do sistema

$$
\left\{\begin{array}{l}
\varepsilon x \psi_{0}^{\prime}(\varepsilon, x)+A_{0}(\varepsilon) \psi_{0}(\varepsilon, x)=g(\varepsilon, x) \\
\varepsilon x \psi_{k}^{\prime}(\varepsilon, x)+A_{0}(\varepsilon) \psi_{k}(\varepsilon, x)=B(\varepsilon, x) \psi_{k-1}(\varepsilon, x) \quad(k \geq 1)
\end{array} .\right.
$$

Observe que se somarmos todas as equações em (4.3.52) e levarmos em conta que $A(\varepsilon, x)=A_{0}(\varepsilon)-B(\varepsilon, x)$, temos que $\sum_{k=0}^{\infty} \psi_{k}(\varepsilon, x)=\phi(\varepsilon, x)$ satisfaz a equação

$$
\varepsilon x \phi^{\prime}(\varepsilon, x)+A(\varepsilon, x) \phi(\varepsilon, x)=g(\varepsilon, x)
$$

- compare (4.3.53) com (4.3.46). Note também, por (4.3.47), (4.3.12) e (4.3.13), que $A_{0}(\varepsilon)=\left.A(\varepsilon, x)\right|_{x=0}=1 \mathrm{e}$

$$
B(\varepsilon, x)=\Upsilon x \phi_{0}(\varepsilon, x)=\Upsilon \sum_{n=1}^{\infty} a_{n-1}(\varepsilon) x^{n}
$$

e portanto $A_{n}(\varepsilon)=-\Upsilon a_{n-1}(\varepsilon)$ para $n \geq 1$. Enunciamos:

Lema 4.3.7 Assumamos que $g(\varepsilon, x)$ admite a expansão 


$$
g(\varepsilon, x)=\sum_{n=0}^{\infty} g_{n}(\varepsilon) x^{n}
$$

e que sua série majorante

$$
G(x)=\sum_{n=0}^{\infty} \sup _{\varepsilon \in S(0, \gamma ; E)}\left|g_{n}(\varepsilon)\right| x^{n}
$$

seja convergente para $x \in D_{\sigma}(0), \sigma<\kappa /(1+\Upsilon c \beta \kappa / 2)$, com $\gamma$, E e $\kappa$ como no Lema 4.3.3 e c definida em (4.3.15). Então existe uma única seqüência de funções $\left(\psi_{k}(\varepsilon, x)\right)_{k \geq 0}$ analíticas em $S(0, \gamma ; E) \times D_{\sigma}(0)$ que satisfaz $(4.3 .52)$. Cada $\psi_{k}(\varepsilon, x)$ possui um zero de ordem $k$ em $x=0$ e vale

$$
\sum_{k=0}^{\infty} \psi_{k}(\varepsilon, x)=\phi(\varepsilon, x)<<\frac{c G(x)}{1-c \Omega(x)}
$$

sendo

$$
\Omega(x)=\sum_{n=1}^{\infty} \sup _{\varepsilon \in S(0, \gamma ; E)}\left|A_{n}(\varepsilon)\right| x^{n} .
$$

Além disso, $\phi(\varepsilon, x)$ é a única solução de (4.3.53) analítica em $S(0, \gamma ; E) \times D_{\sigma}(0)$.

Demonstração. Expandindo para cada $k \geq 0$

$$
\psi_{k}(\varepsilon, x)=\sum_{n=k}^{\infty} \varpi_{n, k}(\varepsilon) x^{n}
$$

e substituindo em (4.3.52), somos conduzidos a

$$
\begin{gathered}
(\varepsilon n+1) \varpi_{n, 0}(\varepsilon)=g_{n}(\varepsilon) \quad(n \geq 0) \\
(\varepsilon n+1) \varpi_{n, k}(\varepsilon)=-\sum_{m=k-1}^{n-1} A_{n-m}(\varepsilon) \varpi_{m, k-1}(\varepsilon) \quad(1 \leq k \leq n) .
\end{gathered}
$$


Note que para cada $n, k$ fixos, $\varpi_{n, k}(\varepsilon)$ é unicamente determinado em termo dos coeficientes anteriores. Da primeira relação temos

$$
\psi_{0}(\varepsilon, x)=\sum_{n=0}^{\infty} \frac{1}{\varepsilon n+1} g_{n}(\varepsilon) x^{n}
$$

e da segunda

$$
\begin{aligned}
\psi_{k}(\varepsilon, x) & =\sum_{n=k}^{\infty} \frac{-1}{\varepsilon n+1} \sum_{m=k-1}^{n-1} A_{n-m}(\varepsilon) \varpi_{m, k-1}(\varepsilon) x^{n} \\
& =\sum_{m=k-1}^{n-1} \varpi_{m, k-1}(\varepsilon) x^{m} \sum_{n=m+1}^{\infty} \frac{-1}{\varepsilon n+1} A_{n-m}(\varepsilon) x^{n-m} \\
& =\sum_{m=k-1}^{n-1} \varpi_{m, k-1}(\varepsilon) x^{m} \sum_{l=1}^{\infty} \frac{-1}{\varepsilon(l+m)+1} A_{l}(\varepsilon) x^{l} .
\end{aligned}
$$

Definindo para cada $k \geq 0$

$$
\Psi_{k}(x)=\sum_{n=k}^{\infty} \sup _{\varepsilon \in S(0, \gamma ; E)}\left|\varpi_{n, k}(\varepsilon)\right| x^{n}
$$

segue de (4.3.62) e (4.3.63), juntamente com (4.3.15), (4.3.56) e (4.3.58), que

$$
\begin{gathered}
\Psi_{0}(|x|) \leq c G(|x|), \quad \therefore \quad \Psi_{0}(x)<<c G(x), \\
\Psi_{k}(|x|) \leq c \Omega(|x|) \Psi_{k-1}(|x|), \quad \therefore \quad \Psi_{k}(x)<<c \Omega(x) \Psi_{k-1}(x) \quad(k \geq 1) .
\end{gathered}
$$

Uma vez que $\psi_{k}(\varepsilon, x)<<\Psi_{k}(x)$ vale para cada $k \geq 0$ e $(\varepsilon, x) \in S(0, \gamma ; E) \times D_{\sigma}(0)$, segue de (4.3.65) e (4.3.66) que $\psi_{k}(\varepsilon, x)<<(c \Omega(x))^{k} c G(x)$ para cada $k \geq 0$ nesse mesmo domínio. Note que a condição $\sigma<\kappa /(1+\Upsilon c \beta \kappa / 2)$ garante que $|\Omega(x)|<1 / c$ para todo $x \in D_{\sigma}(0):$ do fato que $A_{n}(\varepsilon)=-\Upsilon a_{n-1}(\varepsilon)$ para $n \geq 1$ e (4.3.17),

$$
|\Omega(x)|<\Omega(\sigma)=\Upsilon \sum_{n=1}^{\infty} \sup _{\varepsilon \in S(0, \gamma ; E)}\left|a_{n-1}(\varepsilon)\right| \sigma^{n} \leq \frac{\Upsilon \beta \kappa \sigma}{2(\kappa-\sigma)}<\frac{1}{c}
$$


Desse fato e da hipótese que (4.3.56) é convergente em $D_{\sigma}(0)$, temos que $\psi_{k}(\varepsilon, x)$ é majorada por uma série convergente para todo $\varepsilon \in S(0, \gamma ; E)$ e todo $x \in D_{\sigma}(0)$. Procedendo como na demonstração do Lema 4.3.3, o Teste M de Weierstrass e o Teorema 3.3.6 nos levam à conclusão que $\psi_{k}(\varepsilon, x)$, para cada $k \geq 0$, é uma função analítica em $S(0, \gamma ; E) \times D_{\sigma}(0)$.

Por (4.3.67) temos que a série $\sum_{k=0}^{\infty}(c \Omega(x))^{k}$ é convergente em $D_{\sigma}(0)$. Então, pela propriedade 1 mostrada na Proposição 3.3.3, somos conduzidos à (4.3.57). Como (4.3.56) é convergente em $D_{\sigma}(0)$ por hipótese, $\phi(\varepsilon, x)$ é majorada por uma série convergente para todo $\varepsilon \in S(0, \gamma ; E)$ e todo $x \in D_{\sigma}(0)$. Novamente, procedendo como na demonstração do Lema 4.3.3, o Teste M de Weierstrass e o Teorema 3.3.6 nos levam à conclusão que $\phi(\varepsilon, x)$ é uma função analítica em $S(0, \gamma ; E) \times D_{\sigma}(0)$. Como nenhuma outra solução de (4.3.53), regular em $x=0$, existe, esta demonstração está concluída.

Retomemos a demonstração do Lema 4.3.6. Mostraremos por indução que $\phi_{i}(\varepsilon, x)$, para cada $i \geq 0$, é uma função analítica em $S(0, \gamma ; E) \times D_{\sigma}(0)$, e obteremos uma estimativa para a taxa de crescimento de $\left|\phi_{i}(\varepsilon, x)\right|$. A seguinte modificação das normas de Nagumo será utilizada:

$$
\|f\|_{j}=\sup _{x \in D_{\sigma}(0)}\left(d_{\sigma}(x)\right)^{j} \sum_{n=0}^{\infty} \sup _{\varepsilon \in S(0, \gamma ; E)}\left|\frac{1}{n !} \frac{\partial^{n} f(\varepsilon, 0)}{\partial x^{n}}\right||x|^{n}, \quad \text { com } d_{\sigma}(x)=\sigma-|x| .
$$

Claramente $g_{0}(\varepsilon, x)$ (vide (4.3.48)) é analítica em $S(0, \gamma ; E) \times D_{\sigma}(0)$, e $\left\|G_{0}\right\|_{0}=\beta / 2$. Então o Lema 4.3.7 nos diz que $\phi_{0}(\varepsilon, x)$ é analítica em $S(0, \gamma ; E) \times D_{\sigma}(0)$, e por $(4.3 .57)$ temos a estimativa

$$
\left\|\phi_{0}\right\|_{0} \leq \frac{c}{1-c \Omega^{[0]}(\sigma)}\left\|G_{0}\right\|_{0}=\frac{c \beta}{2\left(1-c \Omega^{[0]}(\sigma)\right)} \equiv e_{0}
$$

Pela analiticidade de $\phi_{0}(\varepsilon, x)$ em $S(0, \gamma ; E) \times D_{\sigma}(0)$ segue que $g_{1}(\varepsilon, x)$ (vide (4.3.49)) também é analítica nesse domínio. De (4.3.49) e (4.3.17) temos que

$$
\left\|G_{1}\right\|_{0}=\sum_{n=1}^{\infty} n \sup _{\varepsilon \in S(0, \gamma ; E)}\left|a_{n}(\varepsilon)\right||x|^{n} \leq \frac{\beta \kappa \sigma}{2(\kappa-\sigma)^{2}}
$$


logo $G_{1}(x)$ é convergente para $x \in D_{\sigma}(0)$. Então o Lema 4.3 .7 nos diz que $\phi_{1}(\varepsilon, x)$ é analítica em $S(0, \gamma ; E) \times D_{\sigma}(0)$, e por (4.3.57) temos a estimativa

$$
\begin{aligned}
\left\|\phi_{1}\right\|_{0} & \leq \frac{c}{1-c \Omega^{[1]}(\sigma)}\left\|G_{1}\right\|_{0} \\
& \leq \frac{c}{1-c \Omega^{[1]}(\sigma)}\left\{\frac{\beta \kappa \sigma}{2(\kappa-\sigma)^{2}}\right\} \leq \Delta C_{1} \omega
\end{aligned}
$$

para $\Delta$ e $\omega$ constantes adequadamente escolhidas, e $C_{1}=A$ com $A$ satisfazendo (3.3.1). Assumamos agora, para $l=1,2, \ldots, i-1(\operatorname{com} i \geq 2)$, que $\phi_{l}(\varepsilon, x)$ é analítica em $S(0, \gamma ; E) \times D_{\sigma}(0)$ e que nesse domínio

$$
\left\|\phi_{l}\right\|_{l-1} \leq \Delta C_{l} \omega^{l}
$$

com $C_{l}=A l !^{\lambda} / l^{2}, \lambda \geq 0$ uma constante a ser determinada e $A$ satisfazendo (3.3.1). Então $g_{i}(\varepsilon, x)$ (vide (4.3.50)) é analítica em $S(0, \gamma ; E) \times D_{\sigma}(0)$, e por (4.3.50), (3.3.3) e das propriedades das normas de Nagumo,

$$
\begin{aligned}
\left\|G_{i}\right\|_{i-1} & \leq\|x\|_{0}\left\|\phi_{i-1}^{\prime}\right\|_{i-1}+2\|x\|_{0} \sum_{j=1}^{i-1}\left\|\phi_{j}\right\|_{j-1}\left\|\phi_{i-j}\right\|_{i-j} \\
& \leq \sigma e(i-1)\left\|\phi_{i-1}\right\|_{i-2}+2 \sigma^{2} \sum_{j=1}^{i-1}\left\|\phi_{j}\right\|_{j-1}\left\|\phi_{i-j}\right\|_{i-1-j} \\
& \leq \sigma e(i-1) \Delta C_{i-1} \omega^{i-1}+2 \sigma^{2} \Delta^{2} C_{i} \omega^{i}
\end{aligned}
$$

$\operatorname{logo} G_{i}(x)$ é convergente para $x \in D_{\sigma}(0)$ (pois para todo $x \in D_{\sigma}(0)$ vale a seguinte desigualdade: $\left.\left|G_{i}(x)\right| \leq\left(d_{\sigma}(x)\right)^{-(i-1)}\left\|G_{i}\right\|_{i-1}\right)$. Então o Lema 4.3 .7 nos diz que $\phi_{i}(\varepsilon, x)$ é analítica em $S(0, \gamma ; E) \times D_{\sigma}(0)$, e por (4.3.57) temos a estimativa

$$
\begin{aligned}
\left\|\phi_{i}\right\|_{i-1} & \leq \frac{c}{1-c \Omega^{[i]}(\sigma)}\left\|G_{i}\right\|_{i-1} \\
& \leq \frac{c}{1-c \Omega^{[i]}(\sigma)}\left\{\sigma e(i-1) \Delta C_{i-1} \omega^{i-1}+2 \sigma^{2} \Delta^{2} C_{i} \omega^{i}\right\} \\
& \leq \Delta C_{i} \omega^{i}
\end{aligned}
$$


sendo que a última desigualdade vale contanto que

$$
\frac{c}{1-c \Omega^{[i]}(\sigma)}\left\{\frac{\sigma e}{\omega} \frac{i-1}{i^{\lambda}}\left(\frac{i}{i-1}\right)^{2}+2 \sigma^{2} \Delta\right\} \leq 1
$$

para todo $i \geq 2$, e assim devemos ter $\lambda \geq 1, \Delta$ suficientemente pequeno (em razão do segundo termo) e $\omega>\sigma$ grande o suficiente. Dessa maneira, fixamos $\lambda=1$. Escolhendo $\Delta$ e $\omega$ dentro dessas condições (também levando em conta (4.3.71)), conclui-se que $\phi_{i}(\varepsilon, x)$, para cada $i \geq 0$, é uma função analítica em $S(0, \gamma ; E) \times D_{\sigma}(0)$ e

$$
\sup _{x \in D_{\sigma}(0)}\left(d_{\sigma}(x)\right)^{i-1} \sum_{n=0}^{\infty} \sup _{\varepsilon \in S(0, \gamma ; E)}\left|\frac{1}{n !} \frac{\partial^{n} \phi_{i}(\varepsilon, 0)}{\partial x^{n}}\right||x|^{n} \equiv\left\|\phi_{i}\right\|_{i-1} \leq \Delta \frac{A i !}{i^{2}} \omega^{i}
$$

para cada $i \geq 1$. Por fim, pelas propriedades 4 e 5 das normas de Nagumo temos que para cada $i \geq 1$, todo $\varepsilon \in S(0, \gamma ; E)$ e todo $x \in \overline{D_{\sigma_{1}}(0)}$, com $\sigma_{1}<\sigma$, vale

$$
\left|\phi_{i}(\varepsilon, x)\right| \leq \frac{\sigma}{\left(\sigma-\sigma_{1}\right)^{i}}\left\|\phi_{i}\right\|_{i-1} \leq C i ! \mu^{i}
$$

com $C=\sigma \Delta A$ e $\mu=\omega /\left(\sigma-\sigma_{1}\right)$. A fim de se incluir o caso $i=0$, basta que escolhamos $C=\max \left(e_{0}, \sigma \Delta A\right)$, tendo em vista (4.3.69). Encerra-se assim esta prova.

\subsubsection{Concluindo a Seção: o Teorema}

Teorema 4.3.8 Consideremos a equação (4.3.8), e suponhamos que $\pi<\gamma<2 \pi$. Então, existe um raio $\sigma_{1}>0$ tal que para $x \in \overline{D_{\sigma_{1}}(0)}$ a solução formal $\widehat{\phi}(\varepsilon, x)$ é 1somável na direção $\theta=0$.

Demonstração. Pelo Teorema de Taylor

$$
r_{I}(\varepsilon, x)=\varepsilon^{-I}\left(\phi(\varepsilon, x)-\sum_{i=0}^{I-1} f_{i}(x) \varepsilon^{i}\right)=\frac{I}{\varepsilon^{I}} \int_{0}^{\varepsilon} \phi_{I}(\xi, x)(\varepsilon-\xi)^{I-1} d \xi
$$


sendo que a integral é efetuada ao longo de um caminho interior a $S(0, \gamma ; E)$ que parte de 0 e vai a $\varepsilon$. Tal relação juntamente com o Lema 4.3.6 implica que

$$
\left|r_{I}(\varepsilon, x)\right| \leq C I ! \mu^{I}
$$

vale para todo $I$ e $(\varepsilon, x) \in S^{\prime} \times \overline{D_{\sigma_{1}}(0)}$, com $S^{\prime}$ qualquer subsetor próprio de $S(0, \gamma ; E)$. Adicionalmente, o Lema 4.3.5 afirma que $\widehat{\phi}(\varepsilon, x)$, uma solução formal de (4.3.8), é um elemento de $\mathcal{O}(\sigma)[[\varepsilon]]_{s}$; portanto é um elemento de $\mathcal{O}\left(\sigma_{1}\right)[[\varepsilon]]_{s}$ para qualquer $\sigma_{1}<\sigma$. Tomemos agora $\sigma_{1}<\sigma<\kappa /(1+2 c \beta \kappa) \operatorname{com} \kappa$ como no Lema 4.3 .3 (isto é, satisfazendo (4.3.18)) e $c$ definida em (4.3.15). Portanto, pela Definição 3.1.1, $\widehat{\phi}(\varepsilon, x)$ é uma expansão assintótica de ordem 1 , quando $\varepsilon \rightarrow 0$ no setor $S(0, \gamma ; E)$, de $\phi(\varepsilon, x)$, a qual pelo Lema 4.3.3 é uma solução analítica de (4.3.8) no domínio $S(0, \gamma ; E) \times \overline{D_{\sigma_{1}}(0)}$. Então, como por hipótese $\gamma>\pi, \phi(\varepsilon, x)$ é a única função Gevrey ordem 1 assintoticamente expansiva em $S(0, \gamma ; E)$ que possui $\widehat{\phi}(\varepsilon, x)$ como sua expansão assintótica, e $\widehat{\phi}(\varepsilon, x)$ é 1-somável na direção $\theta=0$ pela Definição 3.1.4. 


\section{Capítulo 5}

\section{O Caso $t>0$ na Criticalidade}

Tendo em mente o que foi aprendido no estudo do caso $N=\infty$, damos início ao estudo da trajetória $O(N)$ crítica, com $N>>1$ finito. Comecemos por apontar um ingrediente que possibilitou todo o desenvolvimento realizado no caso $N=\infty$ : a transformada de

Legendre de $u^{(\infty)}(t, x)=\lim _{N \rightarrow \infty} u^{(N)}(t, x)$ com respeito a $x$. Com essa, e considerando uma derivada parcial em relação a $p$ (a variável conjugada a $x$ ), passamos de uma equação não-linear (equação (2.4) de [41]) para uma linear (equação (2.11) de [41]), que fomos capazes de resolver, e cuja solução nos forneceu informações explícitas.

Posto isso, começaremos este capítulo fazendo a transformada de Legendre da função $u(\varepsilon, t, x)=u^{(N)}(t, x)$ com respeito a $x$. Denotando por $w(\varepsilon, t, p)$ a função obtida, obteremos o problema de valor inicial associado a $v(\varepsilon, t, p)=w_{p}(\varepsilon, t, p)$ (vide (5.1.12)-(5.1.13)). Com essa EDP e EDO em mãos, mostraremos que na criticalidade a série formal de $v(\varepsilon, t, p)$ em potências de $\varepsilon$ é $s$-Gevrey, para todo $p \in \overline{D_{\varsigma}(-1)}$ fixo, sendo que $s>2$ e $\varsigma<1 / 2$. Quanto à questão de ser assintótica, essa ainda permanece em aberto.

\subsection{Transformada de Legendre de $u(\varepsilon, t, x)=u^{(N)}(t, x)$ com Respeito a $x$}

Para lidarmos com o problema de valor inicial (2.3.17)-(2.3.18) aplicamos a transformada de Legendre à função $u^{(N)}(t, x)=u(\varepsilon, t, x)$ com respeito à variável $x$ 


$$
w(\varepsilon, t, p)=\max _{x}(x p-u(\varepsilon, t, x))=\bar{x} p-u(\varepsilon, t, \bar{x})
$$

$\operatorname{com} \bar{x}=\bar{x}(\varepsilon, t, p)$ o valor de $x$ para o qual

$$
p=u_{x}(\varepsilon, t, x)
$$

tem uma solução para todo $t \geq 0$ e $p$ em um certo domínio que depende de $\varepsilon$ e $t$.

Assumindo que a função $w(\varepsilon, t, p)$ é continuamente diferenciável e convexa em relação à variável $p$, a função original $u(\varepsilon, t, x)$ pode ser recuperada pela transformada inversa de Legendre

$$
u(\varepsilon, t, x)=\max _{p}(x p-w(\varepsilon, t, p))=x \bar{p}-w(\varepsilon, t, \bar{p})
$$

sendo que $\bar{p}=\bar{p}(\varepsilon, t, x)$ é tal que resolve

$$
x=w_{p}(\varepsilon, t, p)
$$

para $p$.

Notemos agora que, diferenciando (5.1.1) com respeito a $t$ e $p$, juntamente com a igualdade (5.1.2), somos levados a

$$
\begin{aligned}
w_{t} & =-u_{t}(\varepsilon, t, \bar{x})+\left(p-u_{x}(\varepsilon, t, \bar{x})\right) \bar{x}_{t}=-u_{t} \\
w_{p} & =\bar{x}+\left(p-u_{x}(\varepsilon, t, \bar{x})\right) \bar{x}_{p}=\bar{x} .
\end{aligned}
$$

Portanto $w_{p}$ resolve a equação (5.1.2) para $x$. Temos também de considerar os termos de derivada segunda $u_{x x}(\varepsilon, t, x)$ e $w_{p p}(\varepsilon, t, p)$ : diferenciando em relação a $p$ a segunda relação de (5.1.5), e fazendo uso de (5.1.2), temos que 


$$
\begin{aligned}
w_{p p} & =\bar{x}_{p}+\bar{x}_{p}+p \bar{x}_{p p}-u_{x x}(\varepsilon, t, \bar{x}) \bar{x}_{p}^{2}-u_{x}(\varepsilon, t, \bar{x}) \bar{x}_{p p} \\
& =2 \bar{x}_{p}-u_{x x}(\varepsilon, t, \bar{x}) \bar{x}_{p}^{2}
\end{aligned}
$$

e que

$$
w_{p p}=\bar{x}_{p}
$$

Igualando (5.1.6) e (5.1.7), e assumindo que $u_{x x}(\varepsilon, t, \bar{x}) \neq 0$ e finito, temos a seguinte equação de segundo grau em $\bar{x}_{p}$

$$
u_{x x} \bar{x}_{p}^{2}-\bar{x}_{p}=0
$$

cujas raízes são $\bar{x}_{p}=0$ e $\bar{x}_{p}=1 / u_{x x}$. Entretanto, $w_{p p}(\varepsilon, t, p)$ identicamente nula implica que $w(\varepsilon, t, p)$ é uma função linear em $p$, o que não é desejável. Além disso, devemos optar pela solução que é consistente com a equação

$$
w_{p p} \bar{p}_{x}^{2}-\bar{p}_{x}=0
$$

obtida de forma análoga à (5.1.8) para o potencial $u(\varepsilon, t, x)$, partindo-se de (5.1.3). Concluímos então que

$$
u_{x x}(\varepsilon, t, x) w_{p p}(\varepsilon, t, p)=1
$$

assumindo que ambas funções são não nulas para quaisquer $\varepsilon, x, p$ e $t$.

Utilizando agora as relações (5.1.1), (5.1.5) e (5.1.10), a equação diferencial (2.3.17) fica

$$
w_{t}=-\varepsilon \frac{w_{p}}{w_{p p}}-p+2 p^{2} w_{p}+2 p w_{p}+d w+\hat{p}_{0}(\varepsilon, t)
$$


sendo que o termo $\hat{p}_{0}(\varepsilon, t)$ é dado implicitamente pela equação $w_{p}(\varepsilon, t, p)=0$. Diferenciando ambos os lados de (5.1.11) em relação a $p$, e escrevendo $v=w_{p}=\bar{x}$ temos, por fim, o seguinte problema de valor inicial:

$$
v_{t}-2 p(1+p) v_{p}=\varepsilon\left(-1+\frac{v v_{p p}}{\left(v_{p}\right)^{2}}\right)-1+(d+2+4 p) v
$$

com $v(\varepsilon, 0, p)=\bar{x}(\varepsilon, 0, p)$ o valor de $x$ tal que $p=u_{x}(\varepsilon, 0, x)$ é resolvida, e escrevendo (4.3.8) em termos de $v(\varepsilon, 0, p)$, a solução de

$$
\varepsilon \frac{v(p)}{v^{\prime}(p)}+p-2 p^{2} v(p)+\frac{\beta}{2}=0
$$

\subsection{Série de Potências na Variável $\varepsilon=2 / N$}

Consideremos a série formal de potências

$$
\hat{v}(\varepsilon, t, p)=\sum_{i=0}^{\infty} v^{[i]}(t, p) \varepsilon^{i}
$$

Impondo que essa seja uma solução formal de (5.1.12), obteremos a equação diferencial parcial satisfeita por $v^{[i]}(t, p)$, para cada $i \geq 0$. Também impondo que em $t=0$ essa série formalmente satisfaça $(5.1 .13)$ com

$$
\beta(\varepsilon)=\sum_{i=0}^{\infty} \beta_{i} \varepsilon^{i}
$$

conseguiremos a equação diferencial ordinária satisfeita por $v^{[i]}(0, p)$, para cada $i \geq 0$.

Comentário 5.2.1 Introduzindo a série (5.2.2), daremos um critério para se determinar o valor crítico $\beta_{c}^{[i]}(d)$ na i-ésima ordem da expansão em $\varepsilon$. 


\subsubsection{Equação Satisfeita por Cada Coeficiente $v^{[i]}(t, p)$}

Para (5.1.12): Seja

$$
\varphi(\varepsilon, t, p)=\left(r \circ v_{p}\right)(\varepsilon, t, p), \quad r(x):=\frac{-1}{x}
$$

de modo que $v_{p p} /\left(v_{p}\right)^{2}=\varphi_{p}$. Com essa definição, substituindo (5.2.1) em (5.1.12) e coletando todos os termos de mesma ordem em $\varepsilon$, vemos que para cada $i \geq 0$

$$
v_{t}^{[i]}-2 p(1+p) v_{p}^{[i]}=f_{i}\left(v^{[0]}, \ldots, v^{[i-1]}\right)+(d+2+4 p) v^{[i]},
$$

sendo $f_{0}=-1$,

$$
f_{1}\left(v^{[0]}\right)=-1+\frac{v^{[0]} v_{p p}^{[0]}}{\left(v_{p}^{[0]}\right)^{2}}=-1+v^{[0]} \varphi_{p}^{[0]}
$$

e para $i \geq 2$

$$
f_{i}\left(v^{[0]}, \ldots, v^{[i-1]}\right)=\sum_{j=0}^{i-1} v^{[i-1-j]} \varphi_{p}^{[j]},
$$

com

$$
\varphi^{[0]}(t, p)=\left.\varphi(\varepsilon, t, p)\right|_{\varepsilon=0}=\frac{-1}{v_{p}^{[0]}(t, p)}
$$

e para cada $j \geq 1$

$$
\begin{aligned}
\varphi^{[j]}(t, p) & =\left.\frac{1}{j !} \frac{\partial^{j} \varphi(\varepsilon, t, p)}{\partial \varepsilon^{j}}\right|_{\varepsilon=0}=\left.\frac{1}{j !}\left(r \circ v_{p}\right)^{(j)}(\varepsilon, t, p)\right|_{\varepsilon=0} \\
& =\sum_{k=1}^{j} \frac{(-1)^{k+1}}{\left(v_{p}^{[0]}\right)^{k+1}} \sum_{\substack{l_{1}, \ldots, l_{k} \geq 1: \\
l_{1}+\cdots+l_{k}=j}} v_{p}^{\left[l_{1}\right]} \cdots v_{p}^{\left[l_{k}\right]}
\end{aligned}
$$

pela aplicação formal da fórmula de $\operatorname{Scott}(3.3 .15)$ à $\varphi(\varepsilon, t, p)=\left(r \circ v_{p}\right)(\varepsilon, t, p)$. 
Observação 5.2.2 Da referência [41] sabemos que para $d=4$

$$
v^{[0]}(t, p)=\frac{2+p}{2 p^{2}}-\frac{4-\beta_{0}}{4 p^{2}} e^{2 t}-\frac{1+p}{p^{3}}\left\{2 t+\ln \left[1-\left(1-e^{-2 t}\right)(1+p)\right]\right\},
$$

a qual é uma função analítica para $(t, p) \in \mathbb{R}_{+} \times D_{1}(-1)$. Para $\beta_{0}=\beta_{c}^{[0]}(4)=4$, também sabemos que $v_{p}^{[0]}\left(t, p^{\dagger}(t)\right)=0$ para todo $t \geq 0$, sendo $p^{\dagger}(t)$ uma função real valorada $e$ monótona crescente de $t$ com $p^{\dagger}(0)=-4$ e $p^{\dagger}(\infty)=-3 / 2$.

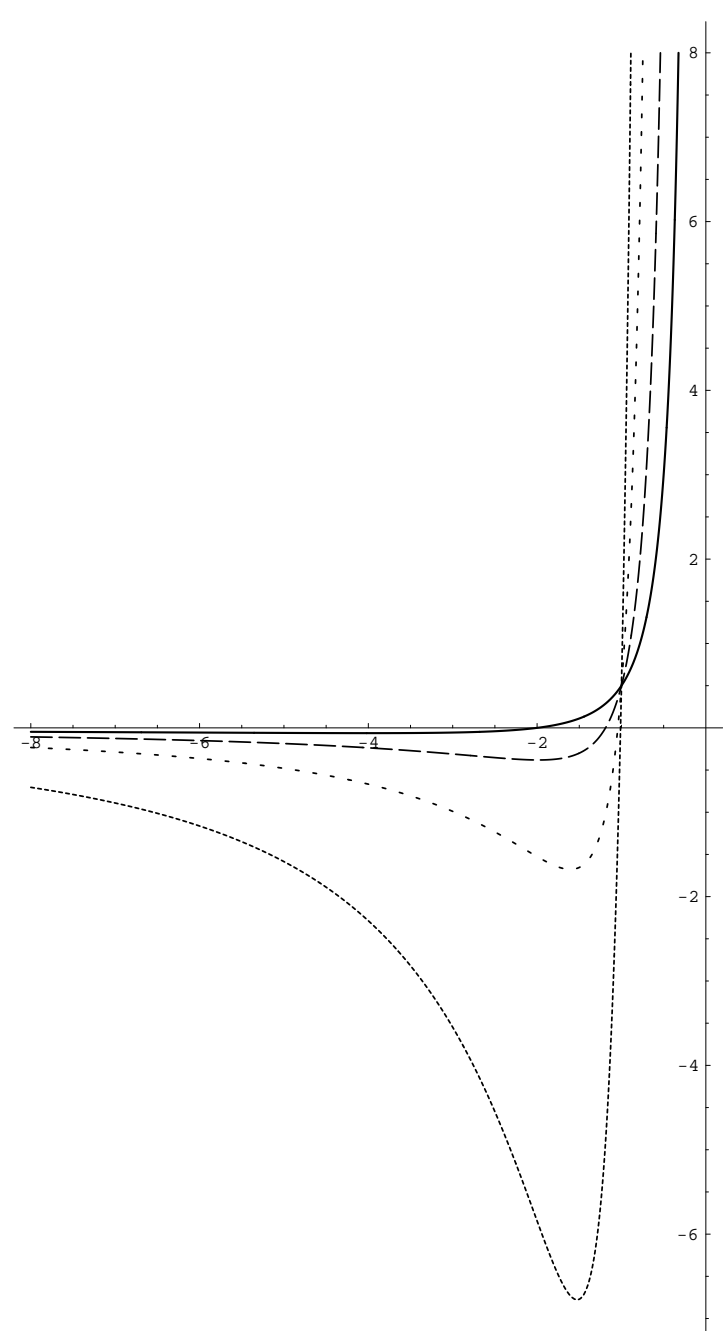

Figura 5.1: Para $p$ real e $\beta_{0}=\beta_{c}^{[0]}(4)=4$, gráfico da função $v^{[0]}(t, p)$ em $t=0$ (linha cheia), $t=10$ (linha com traços médios e próximos), $t=10^{5}$ (linha com traços pequenos e esparços) e $t=10^{20}$ (linha com traços pequenos e próximos). Observe que fixar $\beta_{0}$ no valor crítico $\beta_{c}^{[0]}(4)=4$ faz com que $v^{[0]}(t,-1)=1 / 2$ para todo $t \geq 0$. 
Portanto, $1 / v_{p}^{[0]}(t, p)$ é analítica para $(t, p) \in \mathbb{R}_{+} \times D_{\left|1+p^{\dagger}(t)\right|}(-1)$, e em particular para $(t, p) \in \mathbb{R}_{+} \times D_{1 / 2}(-1)$. Se então suposermos que cada $v^{[l]}(t, p)$, para $l=0,1, \ldots, i-1$, é analítica para $(t, p) \in \mathbb{R}_{+} \times D_{r_{l}}(-1)$, com $r_{l} \leq 1$, segue que $f_{i}\left(v^{[0]}, \ldots, v^{[i-1]}\right)$ é analítica para $t \in \mathbb{R}_{+}$e p em

$$
D_{1 / 2}(-1) \cap \bigcap_{l=0}^{i-1} D_{r_{l}}(-1)
$$

Para (5.1.13): Considerando a definição (5.2.3), substituindo (5.2.1) (com $t=0)$ e (5.2.2) em (5.1.13) e coletando todos os termos de mesma ordem em $\varepsilon$, temos que para cada $i \geq 0$

$$
v_{0, i}(p) \equiv v^{[i]}(0, p)=\frac{1}{4 p^{2}}\left\{g_{i}\left(v_{0,0}, \ldots, v_{0, i-1}\right)+\beta_{i}\right\}
$$

sendo que $g_{0}=2 p$ e para $i \geq 1$

$$
g_{i}\left(v_{0,0}, \ldots, v_{0, i-1}\right)=-2 \sum_{j=0}^{i-1} v_{0, i-1-j} \varphi_{0}^{[j]}
$$

com

$$
\varphi_{0}^{[j]}(p)=\left.\varphi^{[j]}(t, p)\right|_{t=0}
$$

$\varphi^{[0]}(t, p)$ dada por $(5.2 .7)$ e para cada $j \geq 1 \varphi^{[j]}(t, p)$ dada por $(5.2 .8)$.

Observação 5.2.3 Escolhendo $\beta_{0}=\beta_{c}^{[0]}(4)=4$ em $v_{0,0}(p)$ temos

$$
\frac{1}{v_{0,0}^{\prime}(p)}=-\frac{2 p^{3}}{4+p}
$$

que é uma função analítica para $p \in D_{3}(-1)$. Supondo que cada $v_{0, l}(p)$, para $l=$ $0,1, \ldots, i-1$, é analítica para $p \in D_{r_{0, l}}(-1)$, com $r_{0, l} \leq 1$, segue que $g_{i}\left(v_{0,0}, \ldots, v_{0, i-1}\right)$ é analítica para $p$ em 


$$
D_{3}(-1) \cap \bigcap_{l=0}^{i-1} D_{r_{0, l}}(-1)
$$

Uma vez que $r_{0,0}=1$, conclui-se por indução que $v_{0, i}(p)$ é analítica para $p \in D_{1}(-1)$, para todo $i \geq 1-v_{0, i}(p)$ possui um pólo em $p=-4$ e $p=0$.

Por simplicidade, de agora em diante manteremos fixo $d=4$.

Proposição 5.2.4 O problema de valor inicial (5.2.4) e (5.2.11) com $d=4$ possui como solução a função

$$
v^{[i]}(t, p)=\frac{1}{\alpha(p)}\left\{\left(\alpha v_{0, i}\right) \circ \phi_{t}(p)+\int_{0}^{t}\left(\alpha f_{i}\right) \circ \phi_{t-s}(p) d s\right\},
$$

$\operatorname{com} \alpha(p)=2 p^{3} /(1+p)$ e $\phi_{t}(p)=p e^{2 t} /\left(1+p-p e^{2 t}\right)$.

Comentário 5.2.5 Para $d>2$ temos $\alpha_{d}(p)=2 p^{(d+2) / 2} /(1+p)^{(d-2) / 2}$.

Demonstração. Definamos $\vartheta^{[i]}(t, p):=\alpha(p) v^{[i]}(t, p)$. Em termos dessa nova função o problema (5.2.4) e (5.2.11) é escrito como

$$
\begin{gathered}
\vartheta_{t}^{[i]}-2 p(1+p) \vartheta_{p}^{[i]}=\alpha f_{i}\left(v^{[0]}, \ldots, v^{[i-1]}\right) \\
\vartheta_{0, i}(p)=\frac{p}{2(1+p)}\left\{g_{i}\left(v_{0,0}, \ldots, v_{0, i-1}\right)+\beta_{i}\right\},
\end{gathered}
$$

sendo $f_{i}\left(v^{[0]}, \ldots, v^{[i-1]}\right)$ e $g_{i}\left(v_{0,0}, \ldots, v_{0, i-1}\right)$ vistos como funções externas. Tal equação será resolvida pelo método das características (vide [16]): escrevendo $p=p(t)$ e $V(t)=$ $\vartheta^{[i]}(t, p(t)),(5.2 .17)$ fica reduzida ao par de equações diferenciais ordinárias

$$
\begin{aligned}
\dot{p}(t) & =-2 p(t)(1+p(t)) \\
\dot{V}(t) & =\alpha(p(t)) f_{i}\left(v^{[0]}(t, p(t)), \ldots, v^{[i-1]}(t, p(t))\right)
\end{aligned}
$$


com condições iniciais $p(0)=p_{0}$ e $V(0)=\vartheta^{[i]}(0, p(0))=\vartheta_{0, i}\left(p_{0}\right)$. Integrando a primeira equação de (5.2.19)

$$
\int_{p_{0}}^{p} \frac{d p^{\prime}}{p^{\prime}\left(1+p^{\prime}\right)}=\int_{p_{0}}^{p}\left(\frac{1}{p^{\prime}}-\frac{1}{1+p^{\prime}}\right) d p^{\prime}=-2 \int_{0}^{t} d t^{\prime}
$$

somos levados à

$$
p\left(t, p_{0}\right)=\frac{p_{0} e^{-2 t}}{1+p_{0}-p_{0} e^{-2 t}} .
$$

A segunda equação de (5.2.19) também pode ser integrada:

$$
V(t)=V(0)+\int_{0}^{t} \alpha\left(p\left(s, p_{0}\right)\right) f_{i}\left(v^{[0]}\left(s, p\left(s, p_{0}\right)\right), \ldots, v^{[i-1]}\left(s, p\left(s, p_{0}\right)\right)\right) d s .
$$

De acordo com o método das características, para obter a solução $\vartheta^{[i]}(t, p)$ devemos escrever $p_{0}$ como função de $t$ e $p$ e substituir a expressão encontrada em (5.2.21). Assim, resolvendo (5.2.20) para $p_{0}$ temos

$$
\phi_{t}(p):=p_{0}(t, p)=\frac{p e^{2 t}}{1+p-p e^{2 t}}
$$

note que $\phi_{s} \circ \phi_{t}(p)=\phi_{t-s}(p)$. Substituindo essa expressão em (5.2.21) nos dá

$$
\vartheta^{[i]}(t, p)=\vartheta_{0, i} \circ \phi_{t}(p)+\int_{0}^{t}\left(\alpha f_{i}\right) \circ \phi_{t-s}(p) d s
$$

com

$$
\left(\alpha f_{i}\right) \circ \phi_{t-s}(p)=\alpha \circ \phi_{t-s}(p) f_{i}\left(v^{[0]}\left(s, \phi_{t-s}(p)\right), \ldots, v^{[i-1]}\left(s, \phi_{t-s}(p)\right)\right) .
$$

Pela definição de $\vartheta^{[i]}(t, p),(5.2 .16)$ segue imediatamente. 


\subsubsection{A Criticalidade}

Com o caso $N=\infty$ aprendemos que colocar a solução $v^{[0]}(t, p)$ na criticalidade é sinônimo de escolher $\beta_{0}$ de modo que o termo de maior crescimento em $t$ seja cancelado (vide Seção 3.3 de [7]). No caso $d=4$ tal termo é da ordem $e^{2 t}$, como pode ser visto em (5.2.9), tendo sua origem no termo

$$
\frac{\alpha \circ \phi_{t}(p)}{\alpha(p)}=\frac{e^{2 t}}{p^{2}}+\frac{1+p}{p^{2}} \frac{2 p-e^{-2 t}(1+p)}{\left(p-e^{-2 t}(1+p)\right)^{2}} \equiv \frac{e^{2 t}}{p^{2}}+R(t, p) .
$$

Uma vez que esse termo também aparece na expressão (5.2.16), mantem-se a mesma definição de criticalidade: se já tivermos $\beta_{l}=\beta_{c}^{[l]}(4)$ para $l=0,1, \ldots, i-1, \beta_{c}^{[i]}(4)$ é fixada de tal maneira que

$$
\lim _{t \rightarrow \infty} e^{-2 t}\left[\frac{1}{\alpha(p)}\left\{\left(\alpha v_{0, i}\right) \circ \phi_{t}(p)+\int_{0}^{t}\left(\alpha f_{i}\right) \circ \phi_{t-s}(p) d s\right\}\right]=0
$$

Essa pode ser escrita

$$
v_{0, i}(-1)+\int_{0}^{\infty} e^{-2 s} f_{i}\left(v^{[0]}(s,-1), \ldots, v^{[i-1]}(s,-1)\right) d s=0,
$$

haja vista $(5.2 .25)$ e $\lim _{t \rightarrow \infty} \phi_{t}(p)=\lim _{t \rightarrow \infty} \frac{p e^{2 t}}{1+p-p e^{2 t}}=-1$ para todo $p \neq 0$.

A título de ilustração, vejamos o caso $i=1$ : já tendo fixado $\beta_{0}=\beta_{c}^{[0]}(4)=4 \mathrm{em}$ $(5.2 .9)$, temos

$$
\left.v_{0,1}(p)\right|_{p=-1}=\left.\frac{1}{4 p^{2}}\left\{-\frac{2 p(p+2)}{4+p}+\beta_{1}\right\}\right|_{p=-1}=\frac{1}{4}\left\{\frac{2}{3}+\beta_{1}\right\}
$$

e

$$
\left.f_{1}\left(v^{[0]}\right)\right|_{p=-1}=-1+\left.\frac{v^{[0]} v_{p p}^{[0]}}{\left(v_{p}^{[0]}\right)^{2}}\right|_{p=-1}=-1+\frac{e^{-2 t}+3 / 2+6 t}{(3 / 2+2 t)^{2}}
$$

de modo que (5.2.27) nos conduz à 


$$
\beta_{c}^{[1]}(4)=\frac{4}{3}-4 \int_{0}^{\infty} e^{-2 s} \frac{e^{-2 s}+3 / 2+6 s}{(3 / 2+2 s)^{2}} d s=\frac{4}{3}-4 \times 0,41602 \ldots,
$$

sendo que a integral foi efetuada numericamente utilizando-se o software Mathematica.

Definida a noção de criticalidade, estamos em posição de provar duas proposições que dizem respeito ao comportamento dos coeficientes $v^{[i]}(t, p)$ quando $\beta_{i}=\beta_{c}^{[i]}(4)$.

\subsubsection{Existência de uma Única Solução $v^{[i]}(t, p)$ na Criticalidade}

Daremos agora uma prova da existência de uma única solução $v^{[i]}(t, p)$ analítica (para $\left.(t, p) \in \mathbb{R}_{+} \times D_{r_{i}}(-1)\right)$ do problema de valor inicial (5.2.4) e (5.2.11) quando $d=4 \mathrm{e}$ $\beta_{i}=\beta_{c}^{[i]}(4), i=1,2,3, \ldots$. Para tanto, faremos uso do método majorante de Cauchy.

Para enunciarmos os próximos dois teoremas, consideremos as seqüências de números positivos $\left(r_{i}\right)_{i \geq 0}$ e $\left(\sigma_{i}\right)_{i \geq 0}$ tais que

$$
\cdots<r_{i+1}<\sigma_{i}<r_{i}<\cdots<r_{1}<\sigma_{0}<r_{0}<1 / 2
$$

$\lim _{i \rightarrow \infty} \sigma_{i}=\sigma_{\infty}>0$ existe (e portanto $\lim _{i \rightarrow \infty} r_{i}$ também existe) e

$$
\sigma_{1}-\sigma_{\infty}=\sum_{i=1}^{\infty}\left(\sigma_{i}-r_{i+1}\right)+\sum_{i=1}^{\infty}\left(r_{i+1}-\sigma_{i+1}\right)=2 \sum_{i=1}^{\infty} \frac{\delta}{i^{\Delta}}
$$

para algum $\delta>0$ e $\Delta>1$. Note que $\sigma_{1}-\sigma_{\infty}, \delta$ e $\Delta$ não são independentes; note também que

$$
\frac{2 \delta}{\Delta-1} \leq \sigma_{1}-\sigma_{\infty} \leq \frac{2 \delta \Delta}{\Delta-1}
$$

pois $\frac{1}{i^{\Delta}} \geq \int_{i}^{i+1} \frac{d x}{x^{\Delta}}$ para cada $i \geq 1$, e $\frac{1}{i^{\Delta}} \leq \int_{i-1}^{i} \frac{d x}{x^{\Delta}}$ para cada $i \geq 2$.

Teorema 5.2.6 Consideremos o problema de valor inicial (5.2.4) e (5.2.11) com $d=4$, e portanto $v^{[i]}(t, p)$ é dada por (5.2.16). Fixemos $\beta_{i}=\beta_{c}^{[i]}(4)$ para todo $i \geq 0$. Então 


$$
v^{[i]}(t, p)<<-\frac{1+p}{2 p^{3}} \eta_{i} t+H^{[i]}(t, p) \equiv V^{[i]}(t, p)
$$

para cada $i \geq 1$, com $0<\eta_{i}<\infty$ uma constante e $H^{[i]}(t, p)$ uma função analítica para $(t, p) \in \mathbb{R}_{+} \times D_{r_{i}}(-1)$ e tal que $\max _{t \in \mathbb{R}_{+}} \underset{p \in \sup _{\sigma_{i}}(-1)}{ }\left|H^{[i]}(t, p)\right|<\infty$.

Demonstração. Esta demonstração será feita por indução. Mas antes de a iniciarmos, vejamos o caso $i=0$ : fixemos $\beta_{0}=\beta_{c}^{[0]}(4)=4$ em $v^{[0]}(t, p)$. De (5.2.9) vemos que

$$
v^{[0]}(t, p)=-\frac{1+p}{2 p^{3}} \eta_{0} t+H^{[0]}(t, p)=\sum_{n=0}^{\infty} a_{n}^{(0)}(t)(1+p)^{n}
$$

$\operatorname{com} \eta_{0}=4$ e $H^{[0]}(t, p)$ uma função analítica para $(t, p) \in \mathbb{R}_{+} \times D_{1}(-1)$. Note que

$$
a_{0}^{(0)}(t)=1 / 2, \quad a_{1}^{(0)}(t)=3 / 2+2 t
$$

e

$$
a_{n}^{(0)}(t)=\frac{2 n+1}{2}+\left(n^{2}+n\right) t-\frac{1}{2} \sum_{k=1}^{n-1} \frac{k(k+1)}{n-k}\left(1-e^{-2 t}\right)^{n-k} \quad(n \geq 2) .
$$

Temos assim que $a_{n}^{(0)}(t)>0$ para todo $n \geq 0$ e $t \geq 0$.

Verificando (5.2.34) para $i \geq 2$. Fixemos $\beta_{l}=\beta_{c}^{[l]}(4)$ para $l=1, \ldots, i-1$. Assumamos que (5.2.34) está verificada para esses valores de $l$. Então, por (5.2.6)-(5.2.8) e pela Observação 5.2 .2 , temos que $f_{i}\left(v^{[0]}, \ldots, v^{[i-1]}\right)$ é analítica para $(t, p) \in \mathbb{R}_{+} \times D_{r_{i-1}}(-1)$. Um fato de fundamental importância nesta demonstração é o seguinte:

$$
\max _{t \in \mathbb{R}_{+}} \sup _{p \in \bar{D}_{r_{i}}(-1)}\left|f_{i}\left(v^{[0]}, \ldots, v^{[i-1]}\right)\right| \leq M_{i}<\infty
$$

Para mostrá-lo, comecemos por voltar à (5.2.35)-(5.2.37): uma vez que $a_{n}^{(0)}(t)>0$ para todo $n \geq 0$ e $t \geq 0$, a série

$$
v_{p}^{[0]}(t, p)=\sum_{n=0}^{\infty}(n+1) a_{n+1}^{(0)}(t)(1+p)^{n}
$$


também possui coeficientes estritamente positivos para todo $n \geq 0$ e $t \geq 0$. Pela propriedade 4 mostrada na Proposição 3.3.3 segue que

$$
\varphi^{[0]}(t, p)=\frac{-1}{v_{p}^{[0]}(t, p)}<<\frac{-1}{v_{p}^{[0]}(t, p)-2 a_{1}^{(0)}(t)} \equiv \Phi^{[0]}(t, p)
$$

Adicionalmente, pela propriedade 3 mostrada nessa proposição, $v_{p}^{[l]}(t, p)<<V_{p}^{[l]}(t, p)$ para $l=1, \ldots, i-1$. Desses fatos, e considerando as propriedades 1-4 mostradas na citada proposição, vemos que para cada $j=1,2, \ldots, i-1$

$$
\varphi^{[j]}(t, p)<<\sum_{k=1}^{j} \frac{(-1)^{k+1}}{\left(v_{p}^{[0]}-2 a_{1}^{(0)}(t)\right)^{k+1}} \sum_{\substack{l_{1}, \ldots, l_{k} \geq 1: \\ l_{1}+\cdots+l_{k}=j}} V_{p}^{\left[l_{1}\right]} \cdots V_{p}^{\left[l_{k}\right]} \equiv \Phi^{[j]}(t, p) .
$$

Note que tanto $\Phi^{[0]}(t, p)$ como $\Phi^{[j]}(t, p)$ é $O(1 / t)$. Por fim,

$$
f_{i}\left(v^{[0]}, \ldots, v^{[i-1]}\right)<<\sum_{j=0}^{i-2} V^{[i-1-j]} \Phi_{p}^{[j]}+v^{[0]} \Phi_{p}^{[i-1]}
$$

Como a função majorante de $f_{i}\left(v^{[0]}, \ldots, v^{[i-1]}\right)$ é analítica para $(t, p) \in \mathbb{R}_{+} \times D_{r_{i-1}}(-1)$ e é ordem 1 com respeito à variável $t$, conclui-se (5.2.38).

Retomemos a observação sobre o domínio de analiticidade de $f_{i}\left(v^{[0]}, \ldots, v^{[i-1]}\right)$. Dado que $\alpha(p)$ possui um pólo simples em $p=-1, \alpha f_{i}\left(v^{[0]}, \ldots, v^{[i-1]}\right)$ é analítica para $(t, p) \in$ $\mathbb{R}_{+} \times D_{r_{i-1}}(-1) \backslash\{-1\}$ e, em particular, para $(t, p) \in \mathbb{R}_{+} \times D_{r_{i}}(-1) \backslash\{-1\}$. Nesse domínio de $p$ consideramos a expansão

$$
\alpha f_{i}\left(v^{[0]}, \ldots, v^{[i-1]}\right)=\frac{\gamma_{-1}^{(i)}(t)}{1+p}+\sum_{n=0}^{\infty} \gamma_{n}^{(i)}(t)(1+p)^{n}
$$

sendo que (já considerando que $\left.(1+p) \alpha(p)=2 p^{3}\right)$

$$
\begin{gathered}
\gamma_{-1}^{(i)}(t)=\left.\left[2 p^{3} f_{i}\left(v^{[0]}, \ldots, v^{[i-1]}\right)\right]\right|_{p=-1}=-\left.2 f_{i}\left(v^{[0]}, \ldots, v^{[i-1]}\right)\right|_{p=-1} \\
\gamma_{n}^{(i)}(t)=\frac{1}{2 \pi i} \oint_{|1+z|=r_{i}} \frac{2 z^{3} f_{i}\left(v^{[0]}(t, z), \ldots, v^{[i-1]}(t, z)\right)}{(1+z)^{n+2}} d z \quad(n \geq 0) .
\end{gathered}
$$


Pela Observação 5.2.3 sabemos que a condição inicial $v_{0, i}(p)$ é uma função analítica para $p \in D_{1}(-1)$, e portanto (como $r_{i}<1 / 2<1$ )

$$
\sup _{p \in \overline{D_{r_{i}}(-1)}}\left|v_{0, i}(p)\right| \leq M_{0, i}<\infty
$$

A função $\vartheta_{0, i}(p)=\alpha(p) v_{0, i}(p)$ é analítica para $p \in D_{1}(-1) \backslash\{-1\}$ e, em particular, para $p \in D_{r_{i}}(-1) \backslash\{-1\}$. Nesse último domínio possui a expansão

$$
\vartheta_{0, i}(p)=\frac{c_{-1}^{(i)}(0)}{1+p}+\sum_{n=0}^{\infty} c_{n}^{(i)}(0)(1+p)^{n}
$$

com (já considerando que $\left.(1+p) \alpha(p)=2 p^{3}\right)$

$$
\begin{gathered}
c_{-1}^{(i)}(0)=\left.\left[2 p^{3} v_{0, i}(p)\right]\right|_{p=-1}=-\left.2 v_{0, i}(p)\right|_{p=-1} \\
c_{n}^{(i)}(0)=\frac{1}{2 \pi i} \oint_{|1+z|=r_{i}} \frac{2 z^{3} v_{0, i}(z)}{(1+z)^{n+2}} d z \quad(n \geq 0) .
\end{gathered}
$$

Note agora que

$$
1+\phi_{t}(p)=\frac{1+p}{1+p-p e^{2 t}}
$$

é uma função analítica para $(t, p) \in \mathbb{R}_{+} \times D_{1}(-1)$, satisfaz $1+\phi_{t}(-1)=0$,

$$
\sup _{p \in D_{r}(-1)}\left|1+\phi_{t}(p)\right|<\frac{r e^{-2 t}}{1-r\left(1-e^{-2 t}\right)} \leq r
$$

para $r<1$ e todo $t \geq 0$ (isto é, $1+\phi_{t}(p)$ é um mapa contrativo em $D_{r}(-1)$ ), e

$$
\frac{1}{1+\phi_{t}(p)}=\frac{e^{2 t}}{1+p}+1-e^{2 t}
$$

Assim sendo, vemos que a expansão em série de potências de $\left(\alpha f_{i}\right) \circ \phi_{t-s}(p)$ e $\vartheta_{0, i} \circ \phi_{t}(p)$ são bem definidas para todo $t \geq 0$ e $p \in D_{r_{i}}(-1) \backslash\{-1\}$. 
Por (5.2.51) e do fato que para $n \geq 1$

$$
\begin{aligned}
\left(1+\phi_{t}(p)\right)^{n} & =e^{-2 t} \sum_{j_{1}=0}^{\infty}\left(1-e^{-2 t}\right)^{j_{1}}(1+p)^{j_{1}+1} \cdots e^{-2 t} \sum_{j_{n}=0}^{\infty}\left(1-e^{-2 t}\right)^{j_{n}}(1+p)^{j_{n}+1} \\
& =e^{-2 n t} \sum_{j=0}^{\infty}\left(\sum_{\substack{j_{1}, \ldots, j_{n} \geq 0: \\
j_{1}+\cdots+j_{n}=j}} 1\right)\left(1-e^{-2 t}\right)^{j}(1+p)^{j+n} \\
& =e^{-2 n t} \sum_{j=0}^{\infty} \frac{(j+n-1) !}{j !(n-1) !}\left(1-e^{-2 t}\right)^{j}(1+p)^{j+n}
\end{aligned}
$$

concluímos que para $p \in D_{r_{i}}(-1) \backslash\{-1\}$ podemos escrever

$$
\begin{array}{r}
\vartheta^{[i]}(t, p)=\frac{e^{2 t} c_{-1}^{(i)}(0)}{1+p}+\left(1-e^{2 t}\right) c_{-1}^{(i)}(0)+c_{0}^{(i)}(0)+\sum_{n=1}^{\infty} C_{n}^{(i)}(t)(1+p)^{n}+ \\
\int_{0}^{t}\left[\frac{e^{2(t-s)} \gamma_{-1}^{(i)}(s)}{1+p}+\left(1-e^{2(t-s)}\right) \gamma_{-1}^{(i)}(s)+\gamma_{0}^{(i)}(s)+\sum_{n=1}^{\infty} \Gamma_{n}^{(i)}(s, t)(1+p)^{n}\right] d s
\end{array}
$$

com

$$
C_{n}^{(i)}(t)=e^{-2 t}\left(1-e^{-2 t}\right)^{n-1} \sum_{k=0}^{n-1} c_{k+1}^{(i)}(0)\left(\begin{array}{c}
n-1 \\
k
\end{array}\right)\left(\frac{e^{-2 t}}{1-e^{-2 t}}\right)^{k}
$$

e

$$
\Gamma_{n}^{(i)}(s, t)=e^{-2(t-s)}\left(1-e^{-2(t-s)}\right)^{n-1} \sum_{k=0}^{n-1} \gamma_{k+1}^{(i)}(s)\left(\begin{array}{c}
n-1 \\
k
\end{array}\right)\left(\frac{e^{-2(t-s)}}{1-e^{-2(t-s)}}\right)^{k}
$$

Colocando $\vartheta^{[i]}(t, p)$ na Criticalidade: em (5.2.53) observamos ao menos dois termos que crescem exponencialmente com $t$. Tal crescimento deve ser cancelado pela escolha da temperatura inversa $\beta_{i}$ como sendo igual ao valor crítico. Já tendo $\beta_{l}=\beta_{c}^{[l]}(4)$ para $l=0,1, \ldots, i-1, \beta_{c}^{[i]}(4)$ é fixada de tal maneira que

$$
\left.\lim _{t \rightarrow \infty} e^{-2 t}\left[(1+p) \vartheta^{[i]}(t, p)\right]\right|_{p=-1}=0
$$


ou, equivalentemente,

$$
c_{-1}^{(i)}(0)+\int_{0}^{\infty} e^{-2 s} \gamma_{-1}^{(i)}(s) d s=0
$$

Note que, por (5.2.18) e (5.2.46), somente $c_{-1}^{(i)}(0)$ e $c_{0}^{(i)}(0)$ dependem de $\beta_{i}$. Naturalmente que (5.2.27) e (5.2.57) são equivalentes: $c_{-1}^{(i)}(0)=-\left.2 v_{0, i}(p)\right|_{p=-1}($ vide $(5.2 .47))$ e $\gamma_{-1}^{(i)}(t)=-\left.2 f_{i}\left(v^{[0]}, \ldots, v^{[i-1]}\right)\right|_{p=-1}($ vide $(5.2 .43))$. Ponhamos então $\vartheta^{[i]}(t, p)$ na criticalidade: coletando os termos com crescimento exponencial em (5.2.53) e considerando (5.2.57), vemos que

$$
e^{2 t}\left(c_{-1}^{(i)}(0)+\int_{0}^{t} e^{-2 s} \gamma_{-1}^{(i)}(s) d s\right)\left(\frac{1}{1+p}-1\right)=-e^{2 t} \int_{t}^{\infty} e^{-2 s} \gamma_{-1}^{(i)}(s) d s\left(\frac{1}{1+p}-1\right)
$$

Estamos agora em posição de estimar o módulo dos coeficientes de $\vartheta^{[i]}(t, p)$.

Por (5.2.38), (5.2.43)-(5.2.44), e (5.2.45) e (5.2.48), juntamente com a Proposição 3.2 .1 , temos que

$$
\begin{gathered}
\left|\gamma_{-1}^{(i)}(t)\right| \leq 2 M_{i} \equiv \delta_{i}, \\
\left|\gamma_{n}^{(i)}(t)\right| \leq \frac{1}{2 \pi} \oint_{|1+z|=r_{i}} \frac{2|z|^{3}\left|f_{i}\left(v^{[0]}(t, z), \ldots, v^{[i-1]}(t, z)\right)\right|}{|1+z|^{n+2}}|d z| \leq \frac{\Delta_{i}}{r_{i}^{n}} \quad(n \geq 0), \\
\left|c_{n}^{(i)}(0)\right| \leq \frac{1}{2 \pi} \oint_{|1+z|=r_{i}} \frac{2|z|^{3}\left|v_{0, i}(z)\right|}{|1+z|^{n+2}}|d z| \leq \frac{K_{i}}{r_{i}^{n}} \quad(n \geq 0),
\end{gathered}
$$

$\operatorname{com} \delta_{i} \equiv 2 M_{i}, \Delta_{i} \equiv 2 M_{i}\left(1+r_{i}\right)^{3} / r_{i}$ e $K_{i} \equiv 2 M_{0, i}\left(1+r_{i}\right)^{3} / r_{i}$ constantes que não dependem de $t$, dado que $M_{i}$ e $M_{0, i}$ não dependem. De (5.2.58)

$$
\begin{gathered}
\left|-e^{2 t} \int_{t}^{\infty} e^{-2 s} \gamma_{-1}^{(i)}(s) d s\right| \leq \frac{\delta_{i}}{2}, \\
\left|c_{-1}^{(i)}(0)\right|=\left|-\int_{0}^{\infty} e^{-2 s} \gamma_{-1}^{(i)}(s) d s\right| \leq \frac{\delta_{i}}{2} .
\end{gathered}
$$


De (5.2.60) temos (para $\left.p \in D_{r_{i}}(-1)\right)$

$$
\begin{aligned}
\sum_{n=1}^{\infty} C_{n}^{(i)}(t)(1+p)^{n} & <<\sum_{n=1}^{\infty} e^{-2 t}\left(r_{i}+\left(1-r_{i}\right) e^{-2 t}\right)^{n-1} \frac{K_{i}}{r_{i}^{n}}(1+p)^{n} \\
& =\frac{e^{-2 t} K_{i}(1+p)}{r_{i}-\left(r_{i}+\left(1-r_{i}\right) e^{-2 t}\right)(1+p)} \equiv F^{[i]}(t, p)
\end{aligned}
$$

De (5.2.59), juntamente com a integral

$$
\begin{aligned}
\int_{0}^{t} e^{-2(t-s)}\left(r_{i}+\left(1-r_{i}\right) e^{-2(t-s)}\right)^{n-1} d s & =\frac{1}{2} \int_{e^{-2 t}}^{1}\left(r_{i}+\left(1-r_{i}\right) \tau\right)^{n-1} d \tau \\
& =\frac{1-\left(r_{i}+\left(1-r_{i}\right) e^{-2 t}\right)^{n}}{2 n\left(1-r_{i}\right)}
\end{aligned}
$$

temos que (para $\left.p \in D_{r_{i}}(-1)\right)$

$$
\begin{aligned}
\int_{0}^{t} \sum_{n=1}^{\infty} \Gamma_{n}^{(i)}(s, t)(1+p)^{n} d s & <<\sum_{n=1}^{\infty} \frac{1-\left(r_{i}+\left(1-r_{i}\right) e^{-2 t}\right)^{n}}{2 n\left(1-r_{i}\right)} \frac{\Delta_{i}}{r_{i}^{n}}(1+p)^{n} \\
& =\frac{\Delta_{i}}{2\left(1-r_{i}\right)} \ln \left[\frac{r_{i}-\left(r_{i}+\left(1-r_{i}\right) e^{-2 t}\right)(1+p)}{r_{i}-(1+p)}\right] \\
& \equiv G^{[i]}(t, p) .
\end{aligned}
$$

De todos esses fatos segue que, na criticalidade, $(1+p) \vartheta^{[i]}(t, p)$ é majorada por

$$
\frac{\delta_{i}}{2}+(1+p)\left(\left(\delta_{i}+K_{i}\right)+F^{[i]}(t, p)+\left(\delta_{i}+\Delta_{i}\right) t+G^{[i]}(t, p)\right)
$$

que é uma função analítica de $p$ em $D_{r_{i}}(-1)$, uniformemente em $t$. Agora, uma vez que

$$
v^{[i]}(t, p)=\frac{1}{\alpha(p)} \vartheta^{[i]}(t, p)=\frac{1}{2 p^{3}}\left[(1+p) \vartheta^{[i]}(t, p)\right]
$$

e 


$$
\frac{1}{2 p^{3}}=\frac{-1}{4} \sum_{n=0}^{\infty}(n+1)(n+2)(1+p)^{n}<<\frac{-1}{2 p^{3}}
$$

concluímos pela propriedade 2 mostrada na Proposição 3.3.3 que, na criticalidade,

$$
v^{[i]}(t, p)<<-\frac{1+p}{2 p^{3}} \eta_{i} t+H^{[i]}(t, p) \equiv V^{[i]}(t, p)
$$

$\operatorname{com} \eta_{i} \equiv \delta_{i}+\Delta_{i} \mathrm{e}$

$$
H^{[i]}(t, p) \equiv \frac{-\delta_{i}}{4 p^{3}}-\frac{1+p}{2 p^{3}}\left(\left(\delta_{i}+K_{i}\right)+F^{[i]}(t, p)+G^{[i]}(t, p)\right)
$$

uma função analítica para $(t, p) \in \mathbb{R}_{+} \times D_{r_{i}}(-1)$.

Verificando (5.2.34) para $i=1$. Fixemos $\beta_{0}=\beta_{c}^{[0]}(4)=4$. Por $(5.2 .5)$, (5.2.7) e pela Observação 5.2 .2 , temos que $f_{1}\left(v^{[0]}\right)$ é analítica para $(t, p) \in \mathbb{R}_{+} \times D_{r_{0}}(-1)$. Sua dependência em $t$ pode ser vista explicitamente: tanto o numerador $v^{[0]} v_{p p}^{[0]}$ quanto o denominador $\left(v_{p}^{[0]}\right)^{2}$ cresce com $t^{2}$. Então

$$
\max _{t \in \mathbb{R}_{+}} \sup _{p \in \overline{D_{r_{1}}(-1)}}\left|f_{1}\left(v^{[0]}\right)\right| \leq M_{1}<\infty
$$

Agora, dado que $\alpha(p)$ possui um pólo simples em $p=-1, \alpha f_{1}\left(v^{[0]}\right)$ é analítica para $(t, p) \in \mathbb{R}_{+} \times D_{r_{0}}(-1) \backslash\{-1\}$ e, em particular, para $(t, p) \in \mathbb{R}_{+} \times D_{r_{1}}(-1) \backslash\{-1\}$. Nesse domínio de $p$ consideramos a expansão

$$
\alpha f_{1}\left(v^{[0]}\right)=\frac{\gamma_{-1}^{(1)}(t)}{1+p}+\sum_{n=0}^{\infty} \gamma_{n}^{(1)}(t)(1+p)^{n}
$$

sendo que (já considerando que $(1+p) \alpha(p)=2 p^{3}$ )

$$
\begin{gathered}
\gamma_{-1}^{(1)}(t)=\left.\left[2 p^{3} f_{1}\left(v^{[0]}\right)\right]\right|_{p=-1}=-\left.2 f_{1}\left(v^{[0]}\right)\right|_{p=-1} \\
\gamma_{n}^{(1)}(t)=\frac{1}{2 \pi i} \oint_{|1+z|=r_{1}} \frac{2 z^{3} f_{1}\left(v^{[0]}(t, z)\right)}{(1+z)^{n+2}} d z \quad(n \geq 0) .
\end{gathered}
$$


Pela Observação 5.2.3 sabemos que a condição inicial $v_{0,1}(p)$ é uma função analítica para $p \in D_{1}(-1)$, e portanto (como $r_{1}<1 / 2<1$ )

$$
\sup _{p \in \overline{D_{r_{1}}(-1)}}\left|v_{0,1}(p)\right| \leq M_{0,1}<\infty
$$

A função $\vartheta_{0,1}(p)=\alpha(p) v_{0,1}(p)$ é analítica para $p \in D_{1}(-1) \backslash\{-1\}$ e, em particular, para $p \in D_{r_{1}}(-1) \backslash\{-1\}$. Nesse último domínio possui a expansão

$$
\vartheta_{0,1}(p)=\frac{c_{-1}^{(1)}(0)}{1+p}+\sum_{n=0}^{\infty} c_{n}^{(1)}(0)(1+p)^{n}
$$

com (já considerando que $\left.(1+p) \alpha(p)=2 p^{3}\right)$

$$
\begin{gathered}
c_{-1}^{(1)}(0)=\left.\left[2 p^{3} v_{0,1}(p)\right]\right|_{p=-1}=-\left.2 v_{0,1}(p)\right|_{p=-1} \\
c_{n}^{(1)}(0)=\frac{1}{2 \pi i} \oint_{|1+z|=r_{1}} \frac{2 z^{3} v_{0,1}(z)}{(1+z)^{n+2}} d z \quad(n \geq 0) .
\end{gathered}
$$

Por (5.2.49)-(5.2.51), vemos que a expansão em série de potências de $\left(\alpha f_{1}\right) \circ \phi_{t-s}(p)$ e $\vartheta_{0,1} \circ \phi_{t}(p)$ são bem definidas para todo $t \geq 0$ e $p \in D_{r_{1}}(-1) \backslash\{-1\}$.

Daqui por diante devemos proceder exatamente como no caso $i \geq 2$ : tomando $i=1$ em (5.2.53)-(5.2.66) concluímos que, na criticalidade,

$$
v^{[1]}(t, p)<<-\frac{1+p}{2 p^{3}} \eta_{1} t+H^{[1]}(t, p) \equiv V^{[1]}(t, p)
$$

$\operatorname{com} \eta_{1} \equiv \delta_{1}+\Delta_{1} \mathrm{e}$

$$
H^{[1]}(t, p) \equiv \frac{-\delta_{1}}{4 p^{3}}-\frac{1+p}{2 p^{3}}\left(\left(\delta_{1}+K_{1}\right)+F^{[1]}(t, p)+G^{[1]}(t, p)\right)
$$

uma função analítica para $(t, p) \in \mathbb{R}_{+} \times D_{r_{1}}(-1)$.

Fechada a indução, encerra-se a demonstração. 


\subsubsection{Estimativas de Gevrey para $\left|v^{[i]}(t, p)\right|$ na Criticalidade}

Sejam $\left(r_{i}\right)_{i \geq 0}$ e $\left(\sigma_{i}\right)_{i \geq 0}$ as seqüências de números positivos definidas por (5.2.31)-(5.2.33). Enunciamos:

Teorema 5.2.7 Consideremos o problema de valor inicial (5.2.4) e (5.2.11) com $d=4$, e portanto $v^{[i]}(t, p)$ é dada por (5.2.16). Fixemos $\beta_{i}=\beta_{c}^{[i]}(4)$ para todo $i \geq 0$. Então existe uma constante positiva $\mu$ tal que

$$
\sup _{p \in \overline{D_{\sigma_{i}}(-1)}}\left|v^{[i]}(t, p)\right| \equiv\left\|v^{[i]}\right\|_{\sigma_{i}} \leq L\left(t, \sigma_{0}\right) \widetilde{C}_{i} \mu^{i}
$$

vale para cada $i \geq 0$, sendo $\widetilde{C}_{0}$ uma constante que depende de $\sigma_{0}$ e $\widetilde{C}_{i}=\delta A i !^{s} / i^{2+\Delta}$ para $i \geq 1$, com $s \geq 2 \Delta$ e A satisfazendo (3.3.1); para todo $0<\sigma_{0}<1 / 2, L\left(t, \sigma_{0}\right)>0$ é uma função linear de $t$ que satisfaz

$$
\max _{t \in \mathbb{R}_{+}} \frac{L\left(t, \sigma_{0}\right)}{c\left(t, \sigma_{0}\right)} \leq \frac{1}{2},
$$

sendo que

$$
c\left(t, \sigma_{0}\right):=\inf _{p \in D_{\sigma_{0}}(-1)}\left|v_{p}^{[0]}(t, p)\right|>0
$$

exibe crescimento linear em $t$, por (5.2.35).

Demonstração. Em um primeiro momento assumiremos verificadas (5.2.80)-(5.2.81) e mostraremos, por indução, (5.2.79). Cumprida essa etapa, mostraremos (5.2.80) e (5.2.81).

Ao longo desta demonstração usaremos a definição

$$
C_{i}:=\widetilde{C}_{i} /\left(\sigma_{i}-r_{i+1}\right)=\widetilde{C}_{i} /\left(r_{i+1}-\sigma_{i+1}\right)=A i !^{s} / i^{2}
$$

para $i \geq 1$; note que as constantes $\left(C_{i}\right)_{i \geq 1}$ satisfazem (3.3.3) e (3.3.4). 
Verificando (5.2.79) para $i \geq 2$. Fixemos $\beta_{l}=\beta_{c}^{[l]}(4)$ para $l=0,1, \ldots, i-1$. Assumamos que (5.2.79) está verificada para esses valores de $l$. Então, pela Proposição 3.2.2, temos que para cada $l=0,1, \ldots, i-1$

$$
\left\|v_{p}^{[l]}\right\|_{r_{l+1}} \leq \frac{L\left(t, \sigma_{0}\right) \widetilde{C}_{l} \mu^{l}}{\sigma_{l}-r_{l+1}}=L\left(t, \sigma_{0}\right) C_{l} \mu^{l}
$$

De (5.2.8) e utilizando (5.2.80)-(5.2.82) vemos que para cada $j=1,2, \ldots, i-1$

$$
\begin{aligned}
\left\|\varphi^{[j]}\right\|_{r_{j+1}} & \leq \sum_{k=1}^{j}\left\|\frac{1}{v_{p}^{[0]}}\right\|_{\sigma_{0}}^{k+1} \sum_{\substack{l_{1}, \ldots, l_{k} \geq 1: \\
l_{1}+\cdots+l_{k}=j}}\left\|v_{p}^{\left[l_{1}\right]}\right\|_{r_{l_{1}+1}} \cdots\left\|v_{p}^{\left[l_{k}\right]}\right\|_{r_{l_{k}+1}} \\
& \leq \frac{\mu^{j}}{c\left(t, \sigma_{0}\right)} \sum_{k=1}^{j}\left(\frac{L\left(t, \sigma_{0}\right)}{c\left(t, \sigma_{0}\right)}\right)^{k} \sum_{\substack{l_{1}, \ldots, l_{k} \geq 1: \\
l_{1}+\cdots+l_{k}=j}} C_{l_{1}} \cdots C_{l_{k}} \\
& \leq \frac{1}{c\left(t, \sigma_{0}\right)} C_{j} \mu^{j} .
\end{aligned}
$$

Pela Proposição 3.2.2 segue que para cada $j=1,2, \ldots, i-1$

$$
\left\|\varphi_{p}^{[j]}\right\|_{\sigma_{j+1}} \leq \frac{\frac{1}{c\left(t, \sigma_{0}\right)} C_{j} \mu^{j}}{r_{j+1}-\sigma_{j+1}}=\frac{1}{c\left(t, \sigma_{0}\right)} \frac{j^{\Delta}}{\delta} C_{j} \mu^{j}
$$

Notemos também que pela Proposição 3.2.2, por (5.2.7) e (5.2.81)

$$
\left\|\varphi_{p}^{[0]}\right\|_{\sigma_{i}} \leq \frac{\left\|\varphi^{[0]}\right\|_{r_{i}}}{r_{i}-\sigma_{i}} \leq \frac{1 / c\left(t, \sigma_{0}\right)}{r_{i}-\sigma_{i}}
$$

Portanto, de (5.2.6), (5.2.79) (para $l=0,1, \ldots, i-1),(5.2 .80)$ e $\max _{1 \leq l \leq i-2} \widetilde{C}_{l} / C_{l}=\delta$

$$
\begin{aligned}
\left\|f_{i}\right\|_{\sigma_{i}} & \leq\left\|v^{[i-1]}\right\|_{\sigma_{i-1}}\left\|\varphi_{p}^{[0]}\right\|_{\sigma_{i}}+\sum_{j=1}^{i-2}\left\|v^{[i-1-j]}\right\|_{\sigma_{i-1-j}}\left\|\varphi_{p}^{[j]}\right\|_{\sigma_{j+1}}+\left\|v^{[0]}\right\|_{\sigma_{0}}\left\|\varphi_{p}^{[i-1]}\right\|_{\sigma_{i}} \\
& \leq \frac{1}{2}\left(1+(i-2)^{\Delta}+\frac{(i-1)^{\Delta} \widetilde{C}_{0}}{\delta}\right) C_{i-1} \mu^{i-1} \\
& \leq \frac{1}{2}\left(1+\frac{\widetilde{C}_{0}}{\delta}\right)(i-1)^{\Delta} C_{i-1} \mu^{i-1}
\end{aligned}
$$


Antes de estimarmos $\left\|v^{[i]}\right\|_{\sigma_{i}}$ devemos pôr $v^{[i]}(t, p)$ na criticalidade. Como já foi visto, isso é sinônimo de se garantir, sob a condição (5.2.27), o cancelamento do termo de crescimento exponencial em $t$. Com esse propósito, começamos por substituir (5.2.25) em (5.2.16), de modo que

$$
\begin{aligned}
& v^{[i]}(t, p)=\frac{e^{2 t}}{p^{2}}\left(v_{0, i} \circ \phi_{t}(p)+\int_{0}^{t} e^{-2 s} f_{i} \circ \phi_{t-s}(p) d s\right)+ \\
& +R(t, p) v_{0, i} \circ \phi_{t}(p)+\int_{0}^{t} R(t-s, p) f_{i} \circ \phi_{t-s}(p) d s .
\end{aligned}
$$

Como $f_{i}\left(v^{[0]}, \ldots, v^{[i-1]}\right)$ e $v_{0, i}(p)$ são funções analíticas para $(t, p) \in \mathbb{R}_{+} \times D_{r_{i}}(-1)$, elas admitem uma expansão em torno de $p=-1$ :

$$
\begin{gathered}
v_{0, i}=\left.v_{0, i}\right|_{p=-1}+\left.v_{0, i}^{\prime}\right|_{p=-1}(1+p)+\sum_{n=2}^{\infty} a_{n}^{(i)}(0)(1+p)^{n} \\
f_{i}=\left.f_{i}\right|_{p=-1}+\left.\frac{\partial f_{i}}{\partial p}\right|_{p=-1}(1+p)+\sum_{n=2}^{\infty} b_{n}^{(i)}(t)(1+p)^{n}
\end{gathered}
$$

a série de $v_{0, i}(p)$ e $f_{i}\left(v^{[0]}, \ldots, v^{[i-1]}\right)$ a partir de $n=2$ serão denotadas, respectivamente, por $\tilde{v}_{0, i}(p)$ e $\tilde{f}_{i}\left(v^{[0]}, \ldots, v^{[i-1]}\right)$. Considerando essas expansões no primeiro termo de (5.2.87) juntamente com (5.2.27), concluímos que na criticalidade (5.2.16) é dada por

$$
\begin{gathered}
v^{[i]}(t, p)=\frac{e^{2 t}}{p^{2}}\left(\left.v_{0, i}^{\prime}\right|_{p=-1}\left(1+\phi_{t}(p)\right)+\tilde{v}_{0, i} \circ \phi_{t}(p)-\left.\int_{t}^{\infty} e^{-2 s} f_{i}\right|_{p=-1} d s\right)+ \\
+\frac{e^{2 t}}{p^{2}}\left(\left.\int_{0}^{t} e^{-2 s} \frac{\partial f_{i}}{\partial p}\right|_{p=-1}\left(1+\phi_{t-s}(p)\right) d s+\int_{0}^{t} e^{-2 s} \tilde{f}_{i} \circ \phi_{t-s}(p) d s\right)+ \\
+R(t, p) v_{0, i} \circ \phi_{t}(p)+\int_{0}^{t} R(t-s, p) f_{i} \circ \phi_{t-s}(p) d s
\end{gathered}
$$

Com essa expressão em mãos, estamos aptos a obter uma estimativa para $\left\|v^{[i]}\right\|_{\sigma_{i}}$ em termos de $\left\|v_{0, i}\right\|_{\sigma_{i}}$ e $\left\|f_{i}\right\|_{\sigma_{i}}$. Comecemos: de imediato temos que

$$
\left\|-\left.\frac{e^{2 t}}{p^{2}} \int_{t}^{\infty} e^{-2 s} f_{i}\right|_{p=-1} d s\right\|_{\sigma_{i}} \leq \frac{\left\|f_{i}\right\|_{\sigma_{i}}}{2\left(1-\sigma_{i}\right)^{2}}
$$


Do fato que

$$
\sup _{p \in \overline{D_{\sigma}(-1)}} e^{2 t}\left|1+\phi_{t}(p)\right|=\frac{\sigma}{1-\sigma\left(1-e^{-2 t}\right)} \leq \frac{\sigma}{1-\sigma}
$$

para $\sigma<1$ e todo $t \geq 0$, e pela Proposição 3.2.1 (para $n=1$ ), segue que

$$
\begin{gathered}
\left\|\left.\frac{1}{p^{2}} v_{0, i}^{\prime}\right|_{p=-1} e^{2 t}\left(1+\phi_{t}\right)\right\|_{\sigma_{i}} \leq \frac{\left\|v_{0, i}\right\|_{\sigma_{i}}}{\left(1-\sigma_{i}\right)^{3}}, \\
\left\|\left.\frac{1}{p^{2}} \int_{0}^{t} \frac{\partial f_{i}}{\partial p}\right|_{p=-1} e^{2(t-s)}\left(1+\phi_{t-s}\right) d s\right\|_{\sigma_{i}} \leq \frac{t\left\|f_{i}\right\|_{\sigma_{i}}}{\left(1-\sigma_{i}\right)^{3}} .
\end{gathered}
$$

Do fato que para cada $n \geq 2$

$$
\sup _{p \in \overline{D_{\sigma}(-1)}} e^{2 t}\left|1+\phi_{t}(p)\right|^{n}=\frac{\sigma^{n} e^{-2(n-1) t}}{\left(1-\sigma\left(1-e^{-2 t}\right)\right)^{n}} \leq \sigma^{n}
$$

para $\sigma<1 / 2$ e todo $t \geq 0$ (isto é, $e^{2 t}\left(1+\phi_{t}(p)\right)^{n}$ é um mapa contrativo em $\overline{D_{\sigma}(-1)}$ ), segue que $\left\|e^{2 t} \tilde{v}_{0, i} \circ \phi_{t}\right\|_{\sigma_{i}} \leq\left\|\tilde{v}_{0, i}\right\|_{\sigma_{i}}$ e $\left\|e^{2(t-s)} \tilde{f}_{i} \circ \phi_{t-s}\right\|_{\sigma_{i}} \leq\left\|\tilde{f}_{i}\right\|_{\sigma_{i}}$, e como

$$
\left\|\tilde{v}_{0, i}\right\|_{\sigma_{i}} \leq \sup _{p \in \bar{D}_{\sigma_{i}}(-1)}\left(\left|v_{0, i}\right|+\left.\left|v_{0, i}\right|_{p=-1}|+| v_{0, i}^{\prime}\right|_{p=-1}|| 1+p \mid\right) \leq 3\left\|v_{0, i}\right\|_{\sigma_{i}}
$$

$\mathrm{e}$

$$
\left\|\tilde{f}_{i}\right\|_{\sigma_{i}} \leq \sup _{p \in \overline{D_{\sigma_{i}}(-1)}}\left(\left|f_{i}\right|+\left.\left|f_{i}\right|_{p=-1}|+| \frac{\partial f_{i}}{\partial p}\right|_{p=-1}|| 1+p \mid\right) \leq 3\left\|f_{i}\right\|_{\sigma_{i}},
$$

chegamos a

$$
\begin{gathered}
\left\|\frac{e^{2 t}}{p^{2}} \tilde{v}_{0, i} \circ \phi_{t}(p)\right\|_{\sigma_{i}} \leq \frac{3\left\|v_{0, i}\right\|_{\sigma_{i}}}{\left(1-\sigma_{i}\right)^{2}}, \\
\left\|\frac{1}{p^{2}} \int_{0}^{t} e^{2(t-s)} \tilde{f}_{i} \circ \phi_{t-s}(p) d s\right\|_{\sigma_{i}} \leq \frac{3 t\left\|f_{i}\right\|_{\sigma_{i}}}{\left(1-\sigma_{i}\right)^{2}} .
\end{gathered}
$$

Por fim, da expressão (5.2.25) temos

$$
\|R\|_{\sigma_{i}} \leq \frac{\sigma_{i}}{\left(1-\sigma_{i}\right)^{2}} \frac{2+\sigma_{i}\left(2-e^{-2 t}\right)}{\left(1-\sigma_{i}\left(1-e^{-2 t}\right)\right)^{2}} \leq \frac{2 \sigma_{i}\left(1+\sigma_{i}\right)}{\left(1-\sigma_{i}\right)^{4}}
$$


para todo $t \geq 0$, e pela contratividade de $1+\phi_{t}(p)$ (vide (5.2.50))

$$
\left\|v_{0, i} \circ \phi_{t}\right\|_{\sigma_{i}} \leq\left\|v_{0, i}\right\|_{\sigma_{i}} \quad \text { e } \quad\left\|f_{i} \circ \phi_{t}\right\|_{\sigma_{i}} \leq\left\|f_{i}\right\|_{\sigma_{i}} .
$$

Portanto, por (5.2.91)-(5.2.97) concluímos que, na criticalidade,

$$
\left\|v^{[i]}\right\|_{\sigma_{i}} \leq D\left(\sigma_{i}\right)\left\|v_{0, i}\right\|_{\sigma_{i}}+E\left(t, \sigma_{i}\right)\left\|f_{i}\right\|_{\sigma_{i}}
$$

com

$$
\begin{gathered}
D\left(\sigma_{i}\right):=\frac{3}{\left(1-\sigma_{i}\right)^{2}}+\frac{1}{\left(1-\sigma_{i}\right)^{3}}+\frac{2 \sigma_{i}\left(1+\sigma_{i}\right)}{\left(1-\sigma_{i}\right)^{4}} \\
E\left(t, \sigma_{i}\right):=\frac{1}{2\left(1-\sigma_{i}\right)^{2}}+\left[\frac{3}{\left(1-\sigma_{i}\right)^{2}}+\frac{1}{\left(1-\sigma_{i}\right)^{3}}+\frac{2 \sigma_{i}\left(1+\sigma_{i}\right)}{\left(1-\sigma_{i}\right)^{4}}\right] t .
\end{gathered}
$$

Já possuímos uma estimativa para $\left\|f_{i}\right\|_{\sigma_{i}}$; resta-nos uma para $\left\|v_{0, i}\right\|_{\sigma_{i}}$ na criticalidade. Isso é o que faremos agora: estamos trabalhando com a hipótese de indução de que $\beta_{l}=\beta_{c}^{[l]}(4)$ para $l=0,1, \ldots, i-1$ e que (5.2.79) está verificada para esses valores de $l$. Assim, para $t=0$ e cada $l=0,1, \ldots, i-1$

$$
\left\|v_{0, l}\right\|_{\sigma_{l}} \leq L\left(0, \sigma_{0}\right) \widetilde{C}_{l} \mu^{l}
$$

Pela Proposição 3.2.2, semelhantemente ao que fizemos em (5.2.82), temos que para cada $l=0,1, \ldots, i-1$

$$
\left\|v_{0, l}^{\prime}\right\|_{r_{l+1}} \leq \frac{L\left(0, \sigma_{0}\right) \widetilde{C}_{l} \mu^{l}}{\sigma_{l}-r_{l+1}}=L\left(0, \sigma_{0}\right) C_{l} \mu^{l}
$$

Lembrando que $\varphi_{0}^{[j]}(p)=\left.\varphi^{[j]}(t, p)\right|_{t=0}$, de (5.2.8) e utilizando (5.2.80)-(5.2.81) e (5.2.102) vemos que para cada $j=1,2, \ldots, i-1$ 


$$
\begin{aligned}
\left\|\varphi_{0}^{[j]}\right\|_{r_{j+1}} & \leq \sum_{k=1}^{j}\left\|\frac{1}{v_{0,0}^{\prime}}\right\|_{\sigma_{0}}^{k+1} \sum_{\substack{l_{1}, \ldots, l_{k} \geq 1: \\
l_{1}+\cdots, l_{k}=j}}\left\|v_{0, l_{1}}^{\prime}\right\|_{r_{l_{1}+1}} \cdots\left\|v_{0, l_{k}}^{\prime}\right\|_{r_{l_{k}+1}} \\
& \leq \frac{\mu^{j}}{c\left(0, \sigma_{0}\right)} \sum_{k=1}^{j}\left(\frac{L\left(0, \sigma_{0}\right)}{c\left(0, \sigma_{0}\right)}\right)^{k} \sum_{\substack{l_{1}, \ldots, l_{k} \geq 1: \\
l_{1}+\cdots+l_{k}=j}} C_{l_{1}} \cdots C_{l_{k}} \\
& \leq \frac{1}{c\left(0, \sigma_{0}\right)} C_{j} \mu^{j} .
\end{aligned}
$$

Notemos que por $(5.2 .7)$ e $(5.2 .81)$

$$
\left\|\varphi_{0}^{[0]}\right\|_{\sigma_{i}} \leq \frac{1}{c\left(0, \sigma_{0}\right)}
$$

Portanto, de (5.2.12), (5.2.101) (para $l=0,1, \ldots, i-1),(5.2 .80)$ e $\max _{1 \leq l \leq i-2} \widetilde{C}_{l} / C_{l}=\delta$ (lembrando também que $\sigma_{j+1}<r_{j+1}$ para cada $j \geq 0$ )

$$
\begin{aligned}
\left\|g_{i}\right\|_{\sigma_{i}} & \leq 2\left[\left\|v_{0, i-1}\right\|_{\sigma_{i-1}}\left\|\varphi_{0}^{[0]}\right\|_{\sigma_{i}}+\sum_{j=1}^{i-2}\left\|v_{0, i-1-j}\right\|_{\sigma_{i-1-j}}\left\|\varphi_{0}^{[j]}\right\|_{\sigma_{j+1}}+\left\|v_{0,0}\right\|_{\sigma_{0}}\left\|\varphi_{0}^{[i-1]}\right\|_{\sigma_{i}}\right] \\
& \leq\left(\frac{\delta}{(i-1)^{\Delta}}+\delta+\widetilde{C}_{0}\right) C_{i-1} \mu^{i-1} \leq\left(2 \delta+\widetilde{C}_{0}\right) C_{i-1} \mu^{i-1}
\end{aligned}
$$

Agora, como colocamos $v^{[i]}(t, p)$ na criticalidade, (por (5.2.27))

$$
\begin{aligned}
\left|\beta_{i}\right|=\left|\beta_{c}^{[i]}(4)\right| & \leq\left.\left|g_{i}\right|_{p=-1}\left|+4 \int_{0}^{\infty} e^{-2 s}\right| f_{i}\right|_{p=-1} \mid d s \\
& \leq\left\|g_{i}\right\|_{\sigma_{i}}+2\left\|f_{i}\right\|_{\sigma_{i}} .
\end{aligned}
$$

Dessa igualdade e de (5.2.11) concluímos que, na criticalidade,

$$
\begin{aligned}
\left\|v_{0, i}\right\|_{\sigma_{i}} & \leq \frac{1}{4\left(1-\sigma_{i}\right)^{2}}\left\{\left\|g_{i}\right\|_{\sigma_{i}}+\left|\beta_{c}^{[i]}(4)\right|\right\} \\
& \leq \frac{1}{2\left(1-\sigma_{i}\right)^{2}}\left\{\left\|g_{i}\right\|_{\sigma_{i}}+\left\|f_{i}\right\|_{\sigma_{i}}\right\} .
\end{aligned}
$$


Retornando então a (5.2.98) temos que, na criticalidade,

$$
\left\|v^{[i]}\right\|_{\sigma_{i}} \leq \widetilde{D}\left(\sigma_{i}\right)\left\|g_{i}\right\|_{\sigma_{i}}+\widetilde{E}\left(t, \sigma_{i}\right)\left\|f_{i}\right\|_{\sigma_{i}}
$$

com

$$
\widetilde{D}\left(\sigma_{i}\right):=\frac{D\left(\sigma_{i}\right)}{2\left(1-\sigma_{i}\right)^{2}} \quad \text { e } \quad \widetilde{E}\left(t, \sigma_{i}\right):=\frac{D\left(\sigma_{i}\right)}{2\left(1-\sigma_{i}\right)^{2}}+E\left(t, \sigma_{i}\right) .
$$

Considerando as estimativas (5.2.86) e (5.2.105), concluímos que na criticalidade

$$
\left\|v^{[i]}\right\|_{\sigma_{i}} \leq L\left(t, \sigma_{0}\right) \widetilde{C}_{i} \mu^{i}
$$

vale contanto que

$$
\mu \geq\left(\frac{i}{i-1}\right)^{2} \frac{i^{\Delta-s}}{L\left(t, \sigma_{0}\right) \delta}\left[\widetilde{D}\left(\sigma_{i}\right)\left(2 \delta+\widetilde{C}_{0}\right)+\frac{\widetilde{E}\left(t, \sigma_{i}\right)}{2}\left(1+\frac{\widetilde{C}_{0}}{\delta}\right)(i-1)^{\Delta}\right]
$$

para todo $t \geq 0$ e $i \geq 2$. Isso é verdade se pegarmos $\mu$ grande o suficiente e $s \geq 2 \Delta$.

Verificando (5.2.79) para $i=0$. Pela Observação 5.2.2, $v^{[0]}(t, p)$ é uma função analítica para $(t, p) \in \mathbb{R}_{+} \times D_{1}(-1)$. Se considerarmos qualquer $\sigma_{0}<1 / 2$, o Princípio do Máximo Módulo nos diz que o máximo, para $p \in \overline{D_{\sigma_{0}}(-1)}$, de $\left|v^{[0]}(t, p)\right|$ é atingido em algum $p$ na borda de $\overline{D_{\sigma_{0}}(-1)}$. Agora, fixando $\beta_{0}=\beta_{c}^{[0]}(4)=4$ em $v^{[0]}(t, p)$, sabemos por (5.2.35)-(5.2.37) que $a_{n}^{(0)}(t)>0$ para todo $n \geq 0$ e $t \geq 0$. Desses dois fatos segue que

$$
\begin{aligned}
\sup _{p \in D_{\sigma_{0}}(-1)}\left|v^{[0]}(t, p)\right| & =\left.v^{[0]}(t, p)\right|_{1+p=\sigma_{0}} \\
& =\frac{2+p}{2 p^{2}}-\left.\frac{1+p}{p^{3}}\left\{2 t+\ln \left[1-(1+p)\left(1-e^{-2 t}\right)\right]\right\}\right|_{1+p=\sigma_{0}} \\
& =\frac{1+\sigma_{0}}{2\left(1-\sigma_{0}\right)^{2}}+\frac{\sigma_{0}}{\left(1-\sigma_{0}\right)^{3}}\left\{2 t+\ln \left[1-\sigma_{0}\left(1-e^{-2 t}\right)\right]\right\} \\
& \leq \frac{1+\sigma_{0}}{2\left(1-\sigma_{0}\right)^{2}}+\frac{\sigma_{0}}{\left(1-\sigma_{0}\right)^{3}} 2 t:=L\left(t, \sigma_{0}\right) \widetilde{C}_{0}\left(\sigma_{0}\right) .
\end{aligned}
$$


Verificando (5.2.79) para $i=1$. Fixemos $\beta_{0}=\beta_{c}^{[0]}(4)=4$. Por (5.2.5), (5.2.79) (para $i=0),(5.2 .85)$ (para $i=1)$ e $(5.2 .80)$, temos que

$$
\begin{aligned}
\left\|f_{1}\right\|_{\sigma_{1}} & \leq 1+\left\|v^{[0]}\right\|_{\sigma_{0}}\left\|\varphi_{p}^{[0]}\right\|_{\sigma_{1}} \leq 1+L\left(t, \sigma_{0}\right) \widetilde{C}_{0} \frac{1 / c\left(t, \sigma_{0}\right)}{r_{1}-\sigma_{1}} \\
& \leq 1+\frac{\widetilde{C}_{0}}{2\left(r_{1}-\sigma_{1}\right)} .
\end{aligned}
$$

Por (5.2.12) (para $i=1),(5.2 .101)($ para $i=0),(5.2 .104)($ para $i=1)$ e $(5.2 .80)$

$$
\begin{aligned}
\left\|g_{1}\right\|_{\sigma_{1}} & \leq 2\left\|v_{0,0}\right\|_{\sigma_{0}}\left\|\varphi_{0}^{[0]}\right\|_{\sigma_{1}} \leq 2 L\left(0, \sigma_{0}\right) \widetilde{C}_{0} \frac{1}{c\left(0, \sigma_{0}\right)} \\
& \leq \widetilde{C}_{0}
\end{aligned}
$$

Daqui por diante devemos proceder exatamente como no caso $i \geq 2$ : tomando $i=1$ em (5.2.87)-(5.2.109), e considerando (5.2.113) e (5.2.114), concluímos que na criticalidade

$$
\begin{aligned}
\left\|v^{[1]}\right\|_{\sigma_{1}} & \leq \widetilde{D}\left(\sigma_{1}\right)\left\|g_{1}\right\|_{\sigma_{1}}+\widetilde{E}\left(t, \sigma_{1}\right)\left\|f_{1}\right\|_{\sigma_{1}} \\
& \leq L\left(t, \sigma_{0}\right) \widetilde{C}_{1} \mu
\end{aligned}
$$

contanto que

$$
\mu \geq \max _{t \in \mathbb{R}_{+}} \frac{1}{L\left(t, \sigma_{0}\right) \delta A}\left[\widetilde{D}\left(\sigma_{1}\right) \widetilde{C}_{0}+\widetilde{E}\left(t, \sigma_{1}\right)\left(1+\frac{\widetilde{C}_{0}}{2\left(r_{1}-\sigma_{1}\right)}\right)\right]
$$

Verificando (5.2.81). Fixemos $\beta_{0}=\beta_{c}^{[0]}(4)=4$ em $v^{[0]}(t, p)$. Pela Observação 5.2.2 $v_{p}^{[0]}(t, p) \neq 0 \mathrm{em} \mathbb{R}_{+} \times \overline{D_{\sigma_{0}}(-1)}$. Então, o Princípio do Mínimo Módulo nos diz que o mínimo, para $p \in \overline{D_{\sigma_{0}}(-1)}$, de $\left|v_{p}^{[0]}(t, p)\right|$ é atingido em algum $p$ na borda de $\overline{D_{\sigma_{0}}(-1)}$. 
Agora, por (5.2.35)-(5.2.37) a série

$$
v_{p}^{[0]}(t, p)=\sum_{n=0}^{\infty}(n+1) a_{n+1}^{(0)}(t)(1+p)^{n}
$$

possui coeficientes estritamente positivos para todo $n \geq 0$ e $t \geq 0$. Então

$$
\begin{gathered}
c\left(t, \sigma_{0}\right):=\inf _{p \in \overline{D_{\sigma_{0}}(-1)}}\left|v_{p}^{[0]}(t, p)\right|=\left.v_{p}^{[0]}(t, p)\right|_{1+p=-\sigma_{0}} \\
=-\frac{4+p}{2 p^{3}}+\frac{3+2 p}{p^{4}} \ln \left[e^{2 t}-(1+p)\left(e^{2 t}-1\right)\right]+\left.\frac{(1+p)\left(e^{2 t}-1\right)}{p^{3}\left[e^{2 t}-(1+p)\left(e^{2 t}-1\right)\right]}\right|_{1+p=-\sigma_{0}} \\
=\frac{3-\sigma_{0}}{2\left(1+\sigma_{0}\right)^{3}}+\frac{1-2 \sigma_{0}}{\left(1+\sigma_{0}\right)^{4}}\left\{2 t+\ln \left[1+\sigma_{0}\left(1-e^{-2 t}\right)\right]\right\}+\frac{\sigma_{0}\left(1-e^{-2 t}\right)}{\left(1+\sigma_{0}\right)^{3}\left[1+\sigma_{0}\left(1-e^{-2 t}\right)\right]} \\
\geq \frac{3-\sigma_{0}}{2\left(1+\sigma_{0}\right)^{3}}+\frac{1-2 \sigma_{0}}{\left(1+\sigma_{0}\right)^{4}} 2 t .
\end{gathered}
$$

Verificando (5.2.80). Uma vez que temos uma expressão para $L\left(t, \sigma_{0}\right) \widetilde{C}_{0}\left(\sigma_{0}\right)$ e $c\left(t, \sigma_{0}\right)$, falta ajustarmos a constante $\widetilde{C}_{0}\left(\sigma_{0}\right)$ de tal maneira que (5.2.80) é satisfeita. Em outras palavras, temos de garantir que (por (5.2.112) e (5.2.117))

$$
\frac{1}{\widetilde{C}_{0}\left(\sigma_{0}\right)} \max _{t \in \mathbb{R}_{+}} \frac{L\left(t, \sigma_{0}\right) \widetilde{C}_{0}\left(\sigma_{0}\right)}{c\left(t, \sigma_{0}\right)} \leq \frac{1}{\widetilde{C}_{0}\left(\sigma_{0}\right)} \max _{t \in \mathbb{R}_{+}} \chi\left(t, \sigma_{0}\right)=\frac{1}{2}
$$

sendo

$$
\begin{aligned}
\chi\left(t, \sigma_{0}\right) & =\left(\frac{1+\sigma_{0}}{2\left(1-\sigma_{0}\right)^{2}}+\frac{2 \sigma_{0}}{\left(1-\sigma_{0}\right)^{3}} t\right)\left(\frac{3-\sigma_{0}}{2\left(1+\sigma_{0}\right)^{3}}+\frac{2\left(1-2 \sigma_{0}\right)}{\left(1+\sigma_{0}\right)^{4}} t\right)^{-1} \\
& \equiv \frac{\alpha+\beta t}{\gamma+\delta t}
\end{aligned}
$$

Note que o máximo de $\chi\left(t, \sigma_{0}\right)$ com respeito a $t$ é atingido em $t^{*}=0($ se $\alpha / \gamma>\beta / \delta)$ ou em $t^{*}=\infty($ se $\alpha / \gamma<\beta / \delta)$. Então

$$
\max _{t \in \mathbb{R}_{+}} \chi\left(t, \sigma_{0}\right)=\max \left(\frac{\alpha}{\gamma}, \frac{\beta}{\delta}\right) \equiv \chi^{*}\left(\sigma_{0}\right)
$$


e definindo

$$
\widetilde{C}_{0}\left(\sigma_{0}\right):=2 \chi^{*}\left(\sigma_{0}\right)
$$

garantimos a relação (5.2.80). Na figura abaixo mostramos o gráfico da função $\alpha / \gamma$ (linha tracejada) e da função $\beta / \delta$ (linha cheia) para $0<\sigma_{0}<1 / 2 ; \chi^{*}\left(\sigma_{0}\right)$ é o envelope convexo das duas curvas. Note que $\chi^{*}\left(\sigma_{0}\right)>1 / 3$ para todo $0<\sigma_{0}<1 / 2$, e que $\alpha / \gamma$ e $\beta / \delta$ se interceptam em $\sigma_{0}=1-\sqrt{6} / 3=0,183503 \ldots$..

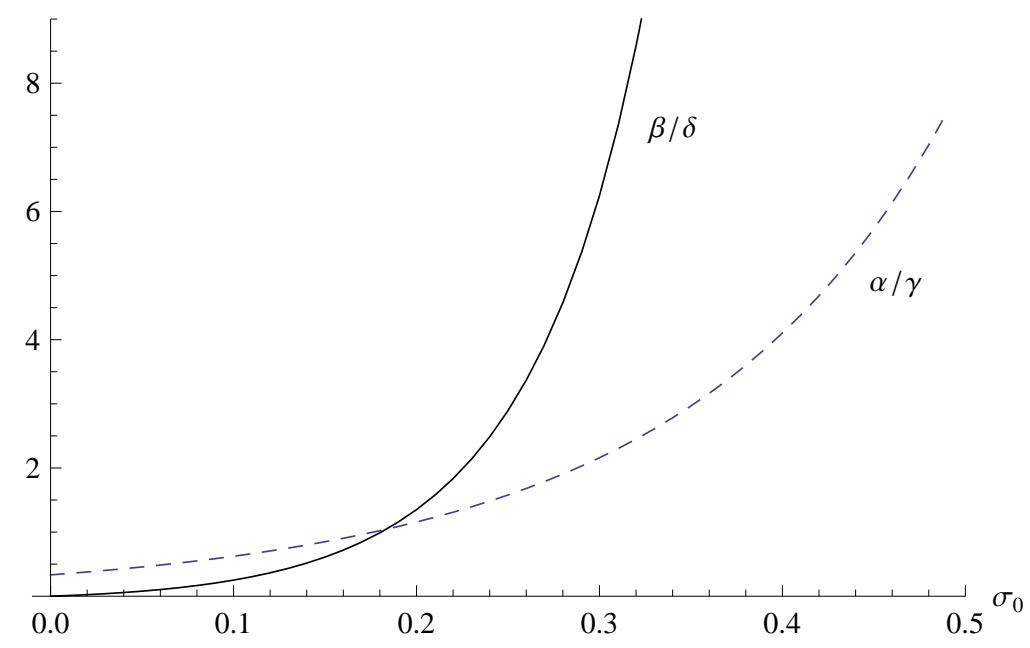

Por fim, podemos definir

$$
L\left(t, \sigma_{0}\right):=\frac{1}{2 \chi^{*}\left(\sigma_{0}\right)}\left(\frac{1+\sigma_{0}}{2\left(1-\sigma_{0}\right)^{2}}+\frac{\sigma_{0}}{\left(1-\sigma_{0}\right)^{3}} 2 t\right) .
$$

Verificadas todas as hipóteses utilizadas, encerramos esta demonstração.

\subsection{Série Assintótica}

Os passo seguinte nesse estudo seria estender para o caso $t>0$ o resultado apresentado na Subseção 4.3.4; todavia, pelo que já tentamos, vimos que essa é uma tarefa bastante difícil, e não sabemos se realmente conseguiremos algum avanço. 


\section{Capítulo 6}

\section{Conclusões e Comentários Finais}

No Capítulo 4 fizemos um estudo amplo e detalhado da condição inicial $u_{x}^{(N)}(0, x)$. Para $N>2$ real e $x$ no plano complexo mostramos que essa é uma função meromorfa, com pólos e zeros localizados sobre o semi-eixo real negativo $\left(\mathbb{R}_{-}^{*}\right)$. Com o limite $N \rightarrow \infty$, os pólos e zeros se adensam sobre o intervalo $(-\infty,-1 /(4 \beta))$. Também mostramos que $\left(u_{x}^{(N)}(0, x)\right)_{N \geq 3}$ é uma seqüência de funções pertence à classe $P$ das funções de Pick, cujo limite também está nessa classe. Embora esses fatos já tenham sido abordados em [7], neste texto o fizemos com maior detalhamento. Também para $N>2$ real e $x$ complexo, mostramos que $u_{x}^{(N)}(0, x)$ é univalente no disco $D_{r}(0)$ de raio $r<p_{1, N}$, com $p_{1, N}$ o pólo de $u_{x}^{(N)}(0, x)$ mais próximo da origem. Em um segundo momento definimos a variável $\varepsilon=2 / N$ e fizemos o estudo de $u_{x}^{(N)}(0, x)=\phi(\varepsilon, x)$ para $\varepsilon$ pertencente a um setor do plano complexo, setor esse que inclui o semi-eixo real positivo $\left(\mathbb{R}_{+}^{*}\right)$, e $x$ no plano complexo. Começamos por obter a equação diferencial (ordinária) satisfeita por $\phi(\varepsilon, x)$. Em posse dessa, mostramos que a solução formal dessa EDO em potências de $\varepsilon$

$$
\widehat{\phi}(\varepsilon, x)=\sum_{i=0}^{\infty} f_{i}(x) \varepsilon^{i}
$$

é 1-somável na direção $\theta=0$. Neste ponto devemos chamar atenção ao fato que $u_{x}^{(N)}(0, x)$ é uma função que envolve a razão de funções de Bessel $J_{\nu}(\xi)$ :

$$
u_{x}^{(N)}(0, x)=\sqrt{\frac{\beta}{4 x}} \frac{i J_{N / 2}(i \sqrt{\beta x} N)}{J_{N / 2-1}(i \sqrt{\beta x} N)} .
$$


Embora funções que envolvam $J_{\nu}(\xi)$ tenham sido amplamente estudadas para $\nu$ real, muito pouco foi feito para $\nu$ complexo.

Iniciamos o Capítulo 5 fazendo a transformada de Legendre de $u^{(N)}(t, x)=u(\varepsilon, t, x)$ com respeito à variável $x$ :

$$
w(\varepsilon, t, p)=\max _{x}(x p-u(\varepsilon, t, x))
$$

Em seguida obtivemos a equação diferencial parcial satisfeita por $v(\varepsilon, t, p)=w_{p}(\varepsilon, t, p)$ (pelas relações da transformada de Legendre tem-se que $v(\varepsilon, t, p)=u_{x}^{-1}(\varepsilon, t, p)$ ). Considerando que a série formal

$$
\hat{v}(\varepsilon, t, p)=\sum_{i=0}^{\infty} v^{[i]}(t, p) \varepsilon^{i}
$$

(formalmente) satisfaça essa $\mathrm{EDP}$, vimos que a cada coeficiente $v^{[i]}(t, p)$ está associado o problema de valor inicial (nos limitamos ao caso $d=4$ )

$$
\begin{gathered}
v_{t}^{[i]}-2 p(1+p) v_{p}^{[i]}=f_{i}\left(v^{[0]}, \ldots, v^{[i-1]}\right)+(6+4 p) v^{[i]} \\
v_{0, i}(p)=\frac{g_{i}\left(v_{0,0}, \ldots, v_{0, i-1}\right)}{4 p^{2}}+\frac{\beta_{c}^{[i]}(4)}{4 p^{2}}
\end{gathered}
$$

sendo que obtivemos uma equação integral para $v^{[i]}(t, p)$. Um critério para determinar o valor de $\beta_{c}^{[i]}(4)$, a temperatura inversa crítica na escala $i$, foi dado: essa é definida pela fórmula (vide (5.2.27) ou, equivalentemente, (5.2.57))

$$
\beta_{c}^{[i]}(4)=-\left.g_{i}\right|_{p=-1}-\left.4 \int_{0}^{\infty} e^{-2 s} f_{i}\right|_{p=-1} d s,
$$

de tal sorte que o crescimento de $O\left(e^{2 t}\right)$ é cancelado. Para o sistema na criticalidade, escrevemos a expansão da equação integral de $\vartheta^{[i]}(t, p)=\alpha(p) v^{[i]}(t, p)$ em torno de $p=-1$, de modo que posteriormente fizemos uso do método majorante de Cauchy para mostrar a existência de uma única solução $v^{[i]}(t, p)$ analítica para $(t, p) \in \mathbb{R}_{+} \times D_{\varsigma}(-1)$. Por fim, mostramos indutivamente que na criticalidade $\hat{v}(\varepsilon, t, p)$ é $s$-Gevrey, para todo $p \in \overline{D_{\varsigma}(-1)}$ fixo, com $s>2$ e $0<\varsigma<1 / 2$. 
Com essa ordem de Gevrey não temos como prosseguir nossa investigação sobre a somabilidade da série formal $\hat{v}(\varepsilon, t, p)$ : pela Definição 3.1.4 é exigido um setor $S(0, \gamma ; E)$ de abertura $\gamma>s \pi$; mas $s \pi>2 \pi$, de modo que avançamos sobre as singularidades que devem se encontrar no semi-eixo real negativo (para a condição inicial $u_{x}(\varepsilon, 0, p)=$ $v^{-1}(\varepsilon, 0, x)$ constatamos a presença de pólos localizados em $\{-1 / n, n \geq 1\}$ no plano complexo $\varepsilon$ ). Com essa observação, vemos a necessidade de se melhorar as estimativas feitas para o crescimentos de $\left|v^{[i]}(t, p)\right|$. Do jeito que as fizemos, o maior problema encontra-se no crescimento exibido por $\left\|\varphi_{p}^{[j]}\right\|_{\sigma_{j+1}}$ : note o fator $j^{\Delta}$ em (5.2.84). No caso de se conseguir melhorar a ordem de Gevrey de $\hat{v}(\varepsilon, t, p)$, o seguinte roteiro deve ser tomado a fim de se verificar a s-somabilidade na direção $\theta=0$ dessa série formal (na criticalidade):

1. mostrar que

$$
\left(\hat{\mathcal{B}}_{s} \hat{v}\right)(\eta, t, p):=\sum_{i=0}^{\infty} v^{[i]}(t, p) \frac{\eta^{i}}{\Gamma(1+i s)}
$$

converge e pode ser analiticamente continuada no setor $S(0, b ; \infty)$ para algum $b>0$;

2. mostrar que para constantes $c$ e $K$ suficientemente grandes vale

$$
\left|\left(\hat{\mathcal{B}}_{s} \hat{v}\right)(\eta, t, p)\right| \leq c \exp \left(K|\eta|^{1 / s}\right), \quad \eta \in S(0, b ; \infty)
$$

Como não dispomos de uma expressão para $v^{[i]}(t, p)$, os itens 1 e 2 devem ser cumpridos com a utilização de séries majorantes. 


\section{Apêndice A}

\section{Apêndices}

\section{A.1 A Classe $P$ das Funções de Pick}

Esta seção do Apêndice é destinada à demonstração do Teorema da Representação Integral Canônica das funções Pick. As definições, fórmulas e teoremas apresentados no tópico Funções Analíticas encontram-se no Capítulo I de [13]; os apresentados nos outros tópicos encontram-se nos capítulos I e II de [12].

Funções Analíticas. Uma função $f: \mathbb{C} \rightarrow \mathbb{C}$ é diferenciável em um ponto $z_{0} \in \mathbb{C}$ se possui derivada

$$
f^{\prime}\left(z_{0}\right)=\lim _{z \rightarrow z_{0}} \frac{f(z)-f\left(z_{0}\right)}{z-z_{0}}
$$

em $z_{0}$. Tal função $f(z)$ é analítica em $z_{0}$ se é diferenciável em todo ponto de uma vizinhança de $z_{0}$.

Uma função complexa $w=f(z)$ pode ser vista geometricamente como um mapa de uma região do plano $z$ em uma região do plano $w$, definido por

$$
\begin{aligned}
w & =f(z) \\
& =u(z)+i v(z),
\end{aligned}
$$


$z=x+i y, u(z)=u(x, y)$ e $v(z)=v(x, y)$ funções real valoradas. Quando $f(z)$ é uma função analítica, suas partes real e imaginária, respectivamente $u$ e $v$, satisfazem as equações de Cauchy-Riemann:

$$
\frac{\partial u}{\partial x}=\frac{\partial v}{\partial y} \quad \text { e } \quad \frac{\partial u}{\partial y}=-\frac{\partial v}{\partial x}
$$

Uma função $g(x, y)$ com segundas derivadas parciais contínuas é dita ser harmônica em um domínio (conjunto aberto conexo no plano complexo $\mathbb{C}$ ) $\mathbb{U}$ se satisfaz a equação de Laplace

$$
\frac{\partial^{2} g}{\partial x^{2}}+\frac{\partial^{2} g}{\partial y^{2}}=0
$$

para todo $x, y \in \mathbb{U}$. Se $f(\zeta)$ é analítica em $\mathbb{U}, u(x, y)$ e $v(x, y)$ satisfazem (A.1.4), e portanto são funções harmônicas em UU. Uma função $v$ é chamada de harmônica conjugada de $u$ se $u$ e $v$ satisfazem as equações de Cauchy-Riemann, isto é, se $u+i v$ é analítica. Quando uma harmônica conjugada existe, ela é única a menos de uma constante aditiva arbitrária. Se $v$ é harmônica conjugada de $u$, então $-u$ é harmônica conjugada de $v$.

Se $g(z)=g(x, y)$ é uma função harmônica sobre um domínio do plano complexo que contém o disco $\left|z-z_{0}\right| \leq r$, então a Propriedade do Valor Médio diz que

$$
g\left(z_{0}\right)=\frac{1}{2 \pi} \int_{0}^{2 \pi} g\left(z_{0}+r e^{i \theta}\right) d \theta .
$$

Sejam $f$ e $g$ funções analíticas respectivamente nos domínios disjuntos $\mathbb{U}$ e $\mathbb{V}$, e um arco $\Gamma$ que é a borda comum a esses dois domínios. Se $f$ e $g$ são ambas definidas e analíticas sobre $\Gamma$, e $f(z)=g(z)$ sobre esse arco, então a função

$$
F(z)=\left\{\begin{array}{lll}
f(z) & \text { para } & z \in \mathbb{U} \cup \Gamma \\
g(z) & \text { para } & z \in \mathbb{V} \cup \Gamma
\end{array}\right.
$$

é analítica em $\mathbb{U} \cup \Gamma \cup \mathbb{V}$. A função $F$ é chamada de continuação analítica de $f$ (ou $g$ ) através de $\Gamma$ para um domínio maior. 
Funções Racionais. Uma função é dita ser racional se pode ser escrita como a razão de dois polinômios.

Seja $f(z)$ uma função racional, e seja

$$
f(z)=\frac{p(z)}{q(z)}
$$

sua representação como a razão entre dois polinômios relativamente primos, isto é, 1 é o maior divisor comum de $p(z)$ e $q(z)$. Seja $m$ o grau de $p(z)$ e $n$ o grau de $q(z)$. Define-se o grau $d$ da função $f(z)$ como

$$
d=\max (m, n)
$$

Para qualquer valor da constante $\lambda$, o número de raízes da equação $f(z)=\lambda$ é exatamente $d$, o grau de $f(z)$. Aqui, naturalmente, devem ser contadas as multiplicidades das raízes, além de uma raíz no infinito, caso exista - para todos os valores finitos de $\lambda$ tais raízes serão todas finitas e simples (multiplicidade 1).

É fácil mostrar que se a função racional $f(z)$ tem grau $m$ e a $g(z)$ tem grau $n$, então tanto a soma quanto o produto dessas funções tem grau no máximo igual $m+n$. Além disso, o grau da derivada $f^{\prime}(z)$ é no máximo duas vezes o grau de $f(z)$.

Se $d$ é o grau da função racional $f(z)$, e tal função se anula em $d+1$ pontos, então $f(z)=0$ identicamente. Deste fato segue que uma função racional de grau $d$ é completamente determinada pelo seu comportamento em $2 d+1$ pontos. Se $f(z)$ e $g(z)$ são funções de grau $d$ que coincidem em $2 d+1$ pontos, então $f(z)-g(z)$ tem ao menos $2 d+1$ zeros e é de grau no máximo $2 d$.

Definição da Classe Pick de Funções. Por classe $P$ das funções de Pick denotamos a classe de funções analíticas no semi-plano superior $\mathbb{H}=\{z=x+i y \in \mathbb{C}: y>0\}$ que têm parte imaginária positiva para $z \in \mathbb{H}$, isto é, $v(z) \geq 0$ se $y>0$. Essa classe forma um cone convexo e é fechada sob composições: para quaisquer $a, b \geq 0$ e $f_{1}, f_{2} \in P$

1. $a f_{1}+b f_{2} \in P$;

2. $f_{1} \circ f_{2} \in P$. 
Seja $f(z)=u(z)+i v(z)$ uma função Pick que é real em algum ponto $z_{0}$ do semiplano superior. Isso significa que a função positiva e harmônica $v(z)$ se anula nesse ponto. Agora, pela Propriedade do Valor Médio das funções harmônicas, segue que $v(z)=0$ identicamente; então $f(z)$ é uma constante real. Vemos assim que uma função Pick não trivial nunca é real em $z \in \mathbb{H}$.

Seja $\left(c_{1}, c_{2}\right)$ um intervalo aberto do eixo real; por $P_{\left(c_{1}, c_{2}\right)}$ denotamos a subclasse de $P$ formada por funções Pick que admitem uma continuação analítica para o semi-plano inferior através do referido intervalo, sendo que essa continuação é por reflexão. Assim, funções nessa classe são reais sobre o intervalo $\left(c_{1}, c_{2}\right)$ e são continuáveis por todo o semi-plano inferior. Uma função não constante em $P$ assume valores reais somente sobre o eixo real. Se $f(z)=u(z)+i v(z)$ está em $P_{\left(c_{1}, c_{2}\right)}, v(x)=0$ para $c_{1}<x<c_{2}$; então $v(x+i y)-v(x)>0$ para $y>0$, e portanto $v_{y}(x) \geq 0$. Por uma das equações de Cauchy-Riemann temos que $u_{x}(x)=v_{y}(x) \geq 0$, e portanto $f(x)=u(x)$ é uma função crescente no intervalo $\left(c_{1}, c_{2}\right)$.

Suponhamos que $f(z)$ é uma função racional pertencente à classe $P_{\left(c_{1}, c_{2}\right)}$. Como dito no parágrafo anterior, $f$ assume valores reais sobre o eixo real, e somente sobre ele. Não é difícil vermos que existe uma constante real $\lambda$ tal que $f(z)-\lambda$ possui $N$ zeros simples e distintos; basta escolhermos $\lambda$ fora do conjunto finito de valores assumidos por $f$ em que a derivada racional se anula. $N$, naturalmente, é o grau da razão $f(z)$. Entre quaisquer dois zeros da função Pick $f-\lambda$ deve existir um pólo, já que a função é sempre crescente. Este também é o caso de uma vizinhança do infinito. Portanto, há $N$ pólos distintos, necessariamente simples, e o resíduo em cada pólo é negativo. É claro que um dos pólos pode estar no infinito, e neste caso o termo correspondente na função racional é do tipo $a z, \operatorname{com} a>0$.

Representação de Funções Pick. Os argumentos apresentados no último parágrafo sobre funções racionais nas classes $P_{\left(c_{1}, c_{2}\right)}$ torna claro que qualquer função racional Pick, real em um intervalo do eixo real, é necessariamente da seguinte forma:

$$
f(z)=a z+b+\sum_{n=1}^{N} \frac{m_{n}}{\alpha_{n}-z}
$$

com $a>0, b$ um número real e $m_{n}>0 ; f(z)$ é uma função meromorfa com pólos de massa $m_{n}$ localizados em $\alpha_{n}$ - por função meromorfa entende-se uma função simples- 
mente valorada (para cada ponto do domínio há um único ponto na imagem) que é analítica em todo domínio com excessão de um conjunto discreto de seu domínio, e em tais singularidades a função deve ir para o infinito polinomialmente, isto é, esses pontos excepcionais devem ser pólos e não singularidades essenciais. Uma simples definição diz que uma função meromorfa é uma função da forma (A.1.7) com $p(z)$ e $q(z)$ funções inteiras (analíticas em todo plano complexo). Portanto, uma função meromorfa pode somente ter ordem finita, pólos isolados e zeros, e nenhuma singularidade essencial em seu domínio.

Enunciaremos agora um teorema que apresenta uma caracterização de funções da classe Pick:

Teorema A.1.1 Uma função $f(z)=u(z)+i v(z)$ pertence à classe Pick se, e somente se, possui a representação integral canônica

$$
f(z)=a z+b+\int_{-\infty}^{\infty}\left(\frac{1}{\lambda-z}-\frac{\lambda}{\lambda^{2}+1}\right) d \mu(\lambda)
$$

sendo que $a=\lim _{y \rightarrow \infty} f(i y) /(i y) \geq 0, b=u(i)$ é real, e $\mu$ é uma medida positiva de Borel sobre $\mathbb{R}$ tal que $\int\left(\lambda^{2}+1\right)^{-1} d \mu(\lambda)<\infty$. Além disso,

$$
\mu\left(\left(x_{1}, x_{2}\right)\right)+\frac{\mu\left(\left\{x_{1}\right\}\right)+\mu\left(\left\{x_{2}\right\}\right)}{2}=\lim _{y \downarrow 0} \frac{1}{\pi} \int_{x_{1}}^{x_{2}} v(x+i y) d x
$$

vale para qualquer intervalo finito $\left(x_{1}, x_{2}\right)$, e determina $\mu$ de maneira única a partir da função $f$.

Há um resultado correspondente para funções harmônicas e positivas no semi-plano superior:

Teorema A.1.2 Qualquer função $v(z)=v(x, y)$ harmônica e positiva no semi-plano superior $\mathbb{H}$ admite uma única representação canônica da forma

$$
v(x, y)=a y+\int_{-\infty}^{\infty} \frac{y}{(\lambda-x)^{2}+y^{2}} d \mu(\lambda)
$$


sendo que $a=\lim _{y \rightarrow \infty} v(0, y) / y \geq 0$ e $\mu$ é uma medida positiva de Borel sobre $\mathbb{R}$ tal que $\int\left(\lambda^{2}+1\right)^{-1} d \mu(\lambda)<\infty$. Reciprocamente, qualquer função dessa forma é harmônica e positiva no semi-plano superior $\mathbb{H}$.

Devemos ressaltar que dado o Teorema A.1.1, podemos deduzir o Teorema A.1.2; e a recíproca também é verdadeira. Para demonstrarmos o Teorema A.1.2, podemos supor $v(x, y)$ dada, harmônica e positiva no semi-plano. Tal função tem uma harmônica conjugada $u(x, y)=u(z)$, determinada a menos de uma constante real aditiva, que é harmônica no semi-plano. Temos então que a função analítica $f(z)=u(z)+i v(z)$ está em $P$ e admite a representação (A.1.10). A função $f(z)$ foi assim determinada a menos da constante real aditiva, e somente o valor $b$ não é determinado por $v(z)$. Se tomarmos a parte imaginária de $f(z)$ na representação (A.1.10), obtemos $v(z)$ na representação (A.1.12), sendo que essa representação é única já que não depende da constante $b$. Por outro lado, o Teorema A.1.1 é obtido do Teorema A.1.2 escrevendo-se $v(z)$, a parte imaginária de $f(z)$, na forma (A.1.12) e notando que a função definida por (A.1.10) e os dados $a$ e $\mu$ com $b=\Re e f(i)$ é uma função analítica no semi-plano superior $\mathbb{H}$ com a dada parte imaginária $(v(z))$ e o valor correto em $z=i$. Então (A.1.10) é uma representação de $f \in P$. Essa representação é única pois $v(z)$, pelo Teorema A.1.2, determina completamente os valores $a$ e $\mu$.

Enunciaremos agora dois teoremas referente a funções definidas no disco unitário:

Teorema A.1.3 Uma função $\phi(w)=\alpha(w)+i \beta(w)$ analítica no disco $|w|<1$ com parte positiva nesse domínio admite uma única representação canônica da forma

$$
\phi(w)=\int_{0}^{2 \pi} \frac{e^{i \theta}+w}{e^{i \theta}-w} d \omega(\theta)+i \beta(0),
$$

com $\beta(0)$ um número real e $\omega$ uma medida positiva de Borel sobre o intervalo [0,2 $\pi]$ com massa total finita. Reciprocamente, qualquer função da forma (A.1.13) é analítica no disco $|w|<1$, e sua parte real é positiva nesse domínio.

Temos também o seguinte teorema:

Teorema A.1.4 Uma função $\alpha(w)$ harmônica e positiva no disco $|w|<1$ possui uma única representação canônica da forma 


$$
\alpha(w)=\alpha\left(r e^{i \theta}\right)=\int_{0}^{2 \pi} \frac{1-r^{2}}{1+r^{2}-2 r \cos (\theta-\varphi)} d \omega(\varphi)
$$

sendo $\omega$ uma medida positiva de Borel sobre o intervalo $[0,2 \pi]$ com massa total finita. Reciprocamente, qualquer função da forma (A.1.14) é harmônica e positiva no disco.

A equivalência entre o Teorema A.1.3 e o Teorema A.1.4 é estabelecida utilizando-se novamente o fato que a função harmônica conjugada é determinada a menos de uma constante real aditiva. Temos também de notar que para $w=r e^{i \varphi}$

$$
\frac{e^{i \theta}+w}{e^{i \theta}-w}=\frac{1-r^{2}-i 2 r \sin (\theta-\varphi)}{1+r^{2}-2 r \cos (\theta-\varphi)} .
$$

Mostraremos neste parágrafo a equivalência entre os Teoremas A.1.1 e A.1.3: a idéia é usar as funções

$$
z(w)=\frac{1}{i} \frac{w+1}{w-1} \quad \text { e } \quad w(z)=\frac{z-i}{z+i},
$$

um par de transformações fracionais lineares que são uma inversa da outra. A função $z(w)$ mapeia o disco unitário no semi-plano superior, enquanto que $w(z)$ mapeia o semiplano superior no disco unitário. Se $\phi(w)=\alpha(w)+i \beta(w)$ é analítica no disco $|w|<1$ com parte real positiva nesse domínio, a função $f(z)=i \phi(w(z))$ está na classe $P$; reciprocamente, se $f(z)$ está na classe $P$, a função $\phi(w)=-i f(z(w))$ é analítica no disco $|w|<1$ com parte real positiva nesse domínio. Assim, as duas classes de funções estão em correspondência um-para-um. Podemos portanto computar a representação canônica de $f(z)$ em $P$ partindo da correspondente representação de $\phi(w)$ : suponhamos $\phi(w)$ dada por (A.1.13); se a medida $d \omega$ tem um ponto de massa $a>0$ em $\theta=0$, separamos essa massa escrevendo (A.1.13) como

$$
\phi(w)=\frac{1+w}{1-w} a-i b+\int_{0}^{2 \pi} \frac{e^{i \theta}+w}{e^{i \theta}-w} d \omega^{\prime}(\theta)
$$


com $d \omega^{\prime}$ a medida obtida de $d \omega$ pela omissão do ponto de massa $a$ em $\theta=0$. Considerando agora $i \phi(w(z))$, os primeiros dois termos ficam $a z+b$. Substituindo a transformação $w(z)$ na integral acima e multiplicando por $i$, essa fica

$$
\int_{0}^{2 \pi} \frac{z \cos \theta / 2-\sin \theta / 2}{z \sin \theta / 2+\cos \theta / 2} d \omega^{\prime}(\theta)
$$

efetuando a mudança de variável $\lambda=-\cot \theta / 2$, a qual leva a circunferência unitária no eixo real com $w=1$ mapeado no infinito, a medida $d \omega^{\prime}$ torna-se uma medida de massa total finita definida sobre o eixo real. Temos assim

$$
f(z)=a z+b+\int_{-\infty}^{\infty} \frac{\lambda z+1}{\lambda-z} d \nu(\lambda)
$$

$\operatorname{com} \int d \nu(\lambda)<\infty$. Por fim, escrevendo

$$
d \nu(\lambda)=\frac{1}{\lambda^{2}+1} d \mu(\lambda)
$$

obtemos a representação canônica (A.1.10). Devemos enfatizar que todo a argumentação é reversível, isto é, se $f(z)$ em $P$ possui a representação (A.1.10), $\phi(w)=-i f(z(w))$ tem a forma (A.1.13).

O que vimos é que os quatro teoremas que enunciamos são equivalentes. Dessa maneira, por conveniência, demonstraremos a validade da representação canônica para o Teorema A.1.4, e a unicidade da representação para o Teorema A.1.1.

Demonstração. Suponhamos que a função $\alpha(w)=\alpha\left(r e^{i \theta}\right)$ é harmônica e positiva em um disco de raio maior ou igual a 1; é portanto limitada e contínua sobre $|w|=1$. Podemos determinar a harmônica conjugada $\beta(w)$ de tal maneira que essa seja nula em $w=0 ; \operatorname{assim} \phi(w)=\alpha(w)+i \beta(w)$ é analítica em um disco de raio maior ou igual a 1 e por conseguinte representada pela série de potências

$$
\sum_{n=0}^{\infty} c_{n} w^{n}
$$


uniforme e absolutamente convergente sobre a circunferência $|w|=1$. A parte real $\alpha(w)$ é portanto dada pela série

$$
\alpha(w)=\frac{\phi(w)+\overline{\phi(w)}}{2}=c_{0}+\frac{1}{2} \sum_{n=1}^{\infty} c_{n} w^{n}+\overline{c_{n}} \bar{w}^{n}
$$

que é uniforme e absolutamente convergente sobre $|w| \leq 1$. Dessa maneira, nossa função admite uma representação da forma

$$
\alpha(r, \theta)=\sum_{n=-\infty}^{\infty} C_{n} r^{|n|} e^{i n \theta}
$$

não é difícil de se ver que $C_{n}$ são os coeficientes de Fourier da função $\alpha(1, \theta)$; então

$$
\alpha(r, \theta)=\frac{1}{2 \pi} \sum_{n=-\infty}^{\infty} r^{|n|} e^{i n \theta} \int_{0}^{2 \pi} \alpha\left(e^{i \varphi}\right) e^{-i n \varphi} d \varphi
$$

Para $r<1$ podemos permutar a ordem de integração e soma para obter

$$
\frac{1}{2 \pi} \int_{0}^{2 \pi} \sum_{n=-\infty}^{\infty} r^{|n|} e^{i n(\theta-\varphi)} \alpha\left(e^{i \varphi}\right) e^{-i n \varphi} d \varphi
$$

que nos conduz a

$$
\alpha(w)=\alpha(r, \theta)=\frac{1}{2 \pi} \int_{0}^{2 \pi} \frac{1-r^{2}}{1+r^{2}-2 r \cos (\theta-\varphi)} \alpha\left(e^{i \varphi}\right) d \varphi
$$

Esta é a representação (A.1.14) para $\alpha(w)$ com a medida $d \omega(\varphi)$ dada pela densidade positiva e limitada $\frac{1}{2 \pi} \alpha\left(e^{i \varphi}\right)$ relativa à medida de Lebesgue sobre o intervalo $[0,2 \pi]$. É importante notarmos que a massa total dessa medida é justamente o valor de $\alpha(w)$ na origem:

$$
\alpha(0)=\frac{1}{2 \pi} \int_{0}^{2 \pi} \alpha\left(e^{i \varphi}\right) d \varphi
$$


O caso geral é uma conseqüência imediata do caso que mostramos: se $\alpha(w)$ é harmônica e positiva em $|w|<1$, a função $\alpha_{\varepsilon}(w)=\alpha(w /(1+\varepsilon)$ ) é harmônica e positiva em $|w|<1+\varepsilon$, e portanto admite a representação (A.1.14) com uma medida positiva $d \omega_{\varepsilon}(\varphi)$ de massa total $\alpha_{\varepsilon}(0)=\alpha(0)$. Conforme $\varepsilon$ se aproxima de 0 , as funções $\alpha_{\varepsilon}(w)$ convergem para $\alpha(w)$ uniformemente em subconjuntos compactos de $|w|<1$, enquanto o sistema de medidas positivas $d \omega_{\varepsilon}$ satisfazem a hipótese do Teorema de Helly (enunciado ao final desta demonstração), já que há um limite uniforme para suas massas. Portanto existe uma seqüência $\varepsilon_{n}$ convergindo para 0 correspondente à seqüência de medidas $d \omega_{n}$ convergindo fracamente para uma medida positiva $d \omega$ tal que

$$
\alpha(w)=\lim _{\varepsilon \rightarrow 0} \alpha_{\varepsilon}(w)=\int_{0}^{2 \pi} \frac{1-r^{2}}{1+r^{2}-2 r \cos (\theta-\varphi)} d \omega(\varphi) .
$$

Mostraremos agora que os elementos $a, b$ e $d \mu$ da representação integral canônica (A.1.10) são unicamente determinados pela função $f(z)$ em $P$ :

Para mostrarmos que $a$ é determinado de modo único, começamos por reescrever (A.1.10) na forma (A.1.19) que tem por vantagem o fato de a medida $d \nu$ ser de massa total finita. Nessa representação $f(i y) /(i y)$ é igual a

$$
a+\frac{b}{i y}+\int_{-\infty}^{\infty} \frac{\lambda^{2}+1+i \lambda(y-1 / y)}{\lambda^{2}+y^{2}} d \nu(\lambda)
$$

claramente o integrando converge pontualmente para 0 quando $y \rightarrow \infty$, e sua parte real e imaginária são uniformemente limitadas por 1 para $y>1$. Portanto, o Teorema da Convergência de Lebesgue garante que o limite da integral é 0 , de tal modo que

$$
\lim _{y \rightarrow \infty} \frac{f(i y)}{i y}=a
$$

Para vermos que $b=u(i)$, basta considerarmos $x=0$ e $y=1$ em

$$
\Re e f(z)=u(x+i y)=a x+b+\int_{-\infty}^{\infty}\left(\frac{\lambda-x}{(\lambda-x)^{2}+y^{2}}-\frac{\lambda}{\lambda^{2}+1}\right) d \mu(\lambda)
$$

Com relação à medida $d \mu$, enunciamos: 
Lema A.1.5 Para qualquer intervalo finito $x_{1}<x<x_{2}$

$$
\mu\left(\left(x_{1}, x_{2}\right)\right)+\frac{\mu\left(\left\{x_{1}\right\}\right)+\mu\left(\left\{x_{2}\right\}\right)}{2}=\lim _{y \downarrow 0} \frac{1}{\pi} \int_{x_{1}}^{x_{2}} v(x+i y) d x
$$

Demonstração. A parte imaginária de (A.1.10) é dada por

$$
v(x+i y)=a y+\int_{-\infty}^{\infty} \frac{y}{(\lambda-x)^{2}+y^{2}} d \mu(\lambda)
$$

com $x \in \mathbb{R}$ e $y>0$ (pelo fato de que $f \in P$ e portanto é uma função analítica no semiplano superior). Integrando os dois lados de (A.1.33) sobre o intervalo $\left(x_{1}, x_{2}\right)$ temos que

$$
\int_{x_{1}}^{x_{2}} v(x+i y) d x=a y\left(x_{2}-x_{1}\right)+\int_{-\infty}^{\infty} \chi_{x_{1}, x_{2}}(y, \lambda) d \mu(\lambda)
$$

com

$$
\begin{aligned}
\chi_{x_{1}, x_{2}}(y, \lambda) & =\int_{x_{1}}^{x_{2}} \frac{y}{(\lambda-x)^{2}+y^{2}} d x \\
& =\arctan \left(\frac{x_{2}-\lambda}{y}\right)-\arctan \left(\frac{x_{1}-\lambda}{y}\right) \\
& \leq \pi .
\end{aligned}
$$

Note que

$$
\lim _{y \downarrow 0} \chi_{x_{1}, x_{2}}(y, \lambda)=\left\{\begin{array}{ll}
0 & \text { se } \lambda<x_{1}<x_{2} \text { ou } x_{1}<x_{2}<\lambda \\
\pi & \text { se } x_{1}<\lambda<x_{2} \\
\pi / 2 & \text { se } \lambda=x_{1} \text { ou } \lambda=x_{2}
\end{array} .\right.
$$

Podemos reescrever (A.1.34) como 


$$
\int_{x_{1}}^{x_{2}} v(x+i y) d x=a y\left(x_{2}-x_{1}\right)+\int_{x_{1}-1}^{x_{2}+1} \chi_{x_{1}, x_{2}}(y, \lambda) d \mu(\lambda)+I(y),
$$

sendo que

$$
\begin{aligned}
I(y)= & \int_{x_{1}}^{x_{2}}\left(\int_{\lambda \leq x_{1}-1} \frac{y}{(\lambda-x)^{2}+y^{2}} d \mu(\lambda)+\int_{\lambda \geq x_{2}+1} \frac{y}{(\lambda-x)^{2}+y^{2}} d \mu(\lambda)\right) d x \\
\leq & \left(x_{2}-x_{1}\right) \int_{\lambda \leq x_{1}-1} \frac{y}{\left(\lambda-x_{1}\right)^{2}+y^{2}} d \mu(\lambda)+ \\
& +\left(x_{2}-x_{1}\right) \int_{\lambda \geq x_{2}+1} \frac{y}{\left(\lambda-x_{2}\right)^{2}+y^{2}} d \mu(\lambda) .
\end{aligned}
$$

Agora, notemos que a função

$$
g(y) \equiv \frac{y}{\left(\lambda-x_{2}\right)^{2}+y^{2}}
$$

atinge seu máximo valor em $y=\lambda-x_{2}$, e portanto

$$
\frac{y}{\left(\lambda-x_{2}\right)^{2}+y^{2}} \geq \frac{1}{\left(\lambda-x_{2}\right)^{2}+1}
$$

para $y<1<\lambda-x_{2}$; e para $\lambda \neq x_{2}$

$$
\lim _{y \downarrow 0} \frac{y}{\left(\lambda-x_{2}\right)^{2}+y^{2}}=0 .
$$

Assim, pelo Teorema da Convergência Dominada,

$$
\lim _{y \downarrow 0} \int_{\lambda \geq x_{2}+1} \frac{y}{\left(\lambda-x_{2}\right)^{2}+y^{2}} d \mu(\lambda)=0 .
$$

Utilizando um argumento similar temos que 


$$
\lim _{y \downarrow 0} \int_{\lambda \leq x_{1}-1} \frac{y}{\left(\lambda-x_{1}\right)^{2}+y^{2}} d \mu(\lambda)=0
$$

o que nos leva à conclusão que

$$
\lim _{y \downarrow 0} I(y)=0 .
$$

Por outro lado, $0 \leq \chi_{x_{1}, x_{2}}(y, \lambda) \leq \pi$ (vide (A.1.35)) e $\int_{x_{1}-1}^{x_{2}+1} \pi d \mu(\lambda)<\infty$; portanto, aplicando o Teorema da Convergência Dominada à (A.1.37) e utilizando (A.1.34), (A.1.36) e (A.1.44), concluímos que

$$
\lim _{y \downarrow 0} \int_{x_{1}}^{x_{2}} v(x+i y) d x=\pi \mu\left(\left(x_{1}, x_{2}\right)\right)+\frac{\pi}{2}\left[\mu\left(\left\{x_{1}\right\}\right)+\mu\left(\left\{x_{2}\right\}\right)\right]
$$

Encerra-se assim a demonstração do lema.

Com o lema demonstrado, completamos a demonstração do teorema.

Teorema A.1.6 (Teorema da Seleção de Helly) Seja $\left\{\omega_{n}\right\}$ uma seqüência de funções não decrescentes sobre um intervalo limitado $[a, b]$, com $\omega_{n}(a)=0$ e $\omega_{n}(b)=1$. Então alguma subseqüência $\left\{\omega_{n_{j}}\right\}$ converge em todo $[a, b]$ para uma função não decrescente $\omega$, e para cada função contínua $g$ sobre $[a, b]$

$$
\lim _{j \rightarrow \infty} \int_{a}^{b} g(t) d \omega_{n_{j}}(t)=\int_{a}^{b} g(t) d \omega(t) .
$$

Demonstração. Vide páginas 22 e 23 de [13].

Passemos agora à questão das funções $f(z)=u(z)+i v(z)$ que podem ser continuadas analiticamente para o semi-plano inferior através de um intervalo $\left(c_{1}, c_{2}\right)$ do eixo real: 
em subintervalos fechados desse intervalo a função $v(x+i y)$ converge uniformemente com $y$ decrescente para uma função limitada e contínua $v(x)$; a medida $d \mu(\lambda)$ aparece portanto como $\frac{1}{\pi} v(\lambda) d \lambda$ nesse subintervalo. Quando a continuação analítica é possível por reflexão, a função $v(x)$ é nula sobre o subintervalo, e portanto $\mu$ não tem massa alguma sobre o intervalo. Por outro lado, se $\mu$ não tem massa alguma sobre o intervalo $\left(c_{1}, c_{2}\right)$, a integral em (A.1.10) faz sentido para todo $z=x+i 0$ no intervalo e claramente é real sobre esse; a função pode ser continuada para o semi-plano inferior por reflexão, e a função continuada também é dada pela fórmula (A.1.10). Estabelecemos assim o seguinte resultado:

Lema A.1.7 Uma função Pick $f(z)$ pertence à classe $P_{\left(c_{1}, c_{2}\right)}$ se, e somente se, a correspondente medida $\mu$ põe massa alguma no intervalo $\left(c_{1}, c_{2}\right)$.

Convergência de Funções Pick. Há uma topologia natural para a classe Pick de funções, a saber, a topologia de convergência uniforme em subconjuntos compactos do semi-plano superior. É óbvio que sob essa topologia $P$ é um espaço métrico completo. É importante estabelecer uma propriedade de compacticidade para subconjuntos de $P$.

Lema A.1.8 Seja $z_{0}=x_{0}+i y_{0}$ um ponto do semi-plano superior, e $\mathcal{I}$ uma família infinita de funções em $P$ uniformemente limitadas em $z_{0}$, isto é, $\left|f\left(z_{0}\right)\right| \leq M$ para toda $f$ em $\mathcal{I}$. Então existe uma seqüência em $\mathcal{I}$ que é convergente.

Lema A.1.9 Seja $\mathcal{I}$ uma família infinita de funções em $P_{\left(c_{1}, c_{2}\right)}$ uniformemente limitadas em algum subintervalo de $\left(c_{1}, c_{2}\right)$. Então existe uma seqüência em $\mathcal{I}$ que converge em $P$ para um limite em $P_{\left(c_{1}, c_{2}\right)}$ e que também converge para esse limite uniformemente em subconjuntos compactos de $\left(c_{1}, c_{2}\right)$.

Lema A.1.10 Uma família infinita de funções positivas e harmônicas no semi-plano superior contém uma seqüência que converge uniformemente em subconjuntos compactos do semi-plano se é uniformemente limitada em algum ponto. Uma seqüência de tais funções, não limitadas em algum ponto fixo, contém uma subseqüência que converge para infinito, isto é, as recíprocas convergem para 0 uniformemente em conjuntos compactos. 


\section{A.2 Frações Continuadas e o Teorema de Worpitzky}

Esta seção do Apêndice é destinada à demonstração do Teorema de Worpitzky. Todas as definições, fórmulas e teoremas aqui apresentados encontram-se nos capítulos I, II e III de [47].

Definições e Fórmulas. Seja a fração continuada

$$
b_{0}+\frac{a_{1}}{b_{1}+\frac{a_{2}}{b_{2}+\frac{a_{3}}{b_{3}+\frac{a_{4}}{b_{4}+\ddots}}}} \text {. }
$$

Os números $a_{k}$ e $b_{k}$ são chamados elementos, e podem ser quaisquer números complexos; a razão $a_{k} / b_{k}$ é o chamado $k$-ésimo quociente parcial, com $a_{k}$ o $k$-ésimo numerador parcial e $b_{k}$ o $k$-ésimo denominador parcial.

A fração continuada truncada no $k$-ésimo quociente parcial

$$
b_{0}+\frac{a_{1}}{b_{1}+\frac{a_{2}}{b_{2}+\frac{a_{3}}{b_{3}+\cdot \cdot+\frac{a_{k}}{b_{k}}}}}=\frac{A_{k}}{B_{k}}
$$

é chamada de $k$-ésimo aproximante, e muitas vezes de $k$-ésimo convergente; $A_{k}$ é o $k$-ésimo numerador, e $B_{k}$ o $k$-ésimo denominador.

Seja a transformação

$$
T_{k}(z)=b_{0}+\frac{a_{1}}{b_{1}+\frac{a_{2}}{b_{2}+\frac{a_{3}}{b_{3}+\cdot \cdot+\frac{a_{k}}{b_{k}+z}}}}=\frac{A_{k-1} z+A_{k}}{B_{k-1} z+B_{k}}
$$

para $k=0,1,2, \ldots$, com $A_{k-1}, A_{k}, B_{k-1}$ e $B_{k}$ independentes de $z$ e tais que podem ser computados por meio das fórmulas de recorrência fundamentais: 


$$
\begin{aligned}
A_{-1} & =1, \quad B_{-1}=0, \quad A_{0}=b_{0}, \quad B_{0}=1, \\
A_{k+1} & =b_{k+1} A_{k}+a_{k+1} A_{k-1}, \\
B_{k+1} & =b_{k+1} B_{k}+a_{k+1} B_{k-1},
\end{aligned}
$$

com as últimas duas relações válidas para $k=0,1,2, \ldots$ O determinante da transformação $T_{k}(z)$ é dado por

$$
\begin{aligned}
\left|\begin{array}{cc}
A_{k-1} & A_{k} \\
B_{k-1} & B_{k}
\end{array}\right| & =\left|\begin{array}{cc}
A_{k-1} & b_{k} A_{k-1}+a_{k} A_{k-2} \\
B_{k-1} & b_{k} B_{k-1}+a_{k} B_{k-2}
\end{array}\right| \\
& =-a_{k}\left|\begin{array}{ll}
A_{k-2} & A_{k-1} \\
B_{k-2} & B_{k-1}
\end{array}\right|
\end{aligned}
$$

de tal maneira que

$$
A_{k-1} B_{k}-A_{k} B_{k-1}=(-1)^{k} a_{0} a_{1} \ldots a_{k}
$$

para $k=0,1,2, \ldots$, sendo que $a_{0}$ pode ser tomado igual à unidade. A fórmula (A.2.6) é chamada fórmula determinante.

Estamos agora em posição de enunciar a seguinte definição:

Definição A.2.1 A fração continuada (A.2.1) é dita convergir ou ser convergente se ao menos um número finito de seus denominadores $B_{n}$ se anulam, e se o limite de sua seqüência de aproximantes

$$
\lim _{k \rightarrow \infty} \frac{A_{k}}{B_{k}}
$$

existe e é finito. De outro modo, a fração continuada é dita divergir ou ser divergente. O valor de uma fração continuada é definido como sendo o limite (A.2.7) de sua seqüência de aproximantes. Nenhum valor é atribuído para uma fração continuada divergente. 
Note as igualdades

$$
\lim _{k \rightarrow \infty} \frac{A_{k}}{B_{k}}=\lim _{k \rightarrow \infty} T_{k}(0)=\lim _{k \rightarrow \infty} T_{k}(\infty)
$$

Devemos observar que se os numeradores parciais $a_{k}$ são todos diferentes de zero então, por (A.2.6), $A_{k}$ e $B_{k}$ não podem ambas se anular; então a existência do limite finito (A.2.7) está garantido, mas um número finito de denominadores $B_{k}$ pode se anular. Assim, neste importante caso, a fração continuada converge se, e somente se, o limite (A.2.7) existe e é finito.

Os elementos $a_{k}$ e $b_{k}$ podem depender de um ou mais parâmetros, ou podem eles próprios serem considerados como variáveis independentes. Em tais casos, interessa-nos naturalmente a questão de convergência uniforme. Fazemos então a seguinte definição:

Definição A.2.2 Se os elementos $a_{k}$ e $b_{k}$ de uma fração continuada são funções de uma ou mais variáveis sobre um certo domínio $\mathcal{D}$, então a fração continuada é dita convergir uniformemente sobre $\mathcal{D}$ se converge para todos os valores da variável ou variáveis em $\mathcal{D}$, e se sua seqüência de aproximantes converge uniformemente sobre $\mathcal{D}$.

Desigualdades Fundamentais. A fração continuada

$$
\frac{1}{1+\frac{a_{2}}{1+\frac{a_{3}}{1+\frac{a_{4}}{1+\ddots}}}}
$$

é dita satisfazer as desigualdades fundamentais se existem números $r_{k} \geq 0$ tais que

$$
r_{k}\left|1+a_{k}+a_{k+1}\right| \geq r_{k} r_{k-2}\left|a_{k}\right|+\left|a_{k+1}\right|
$$

para $k=1,2,3, \ldots$, sendo que poremos $a_{1}=0, r_{0}=0$ e $r_{-1}=0$.

Teorema A.2.3 Se a fração continuada (A.2.9) é tal que satisfaz as desigualdades fundamentais (A.2.10), então seus denominadores $B_{k}$ são diferentes de zero, e os números

$$
\rho_{k}=\frac{-a_{k+1} B_{k-1}}{B_{k+1}}
$$


satisfazem a desigualdade

$$
\left|\rho_{k}\right| \leq r_{k}, \quad k=1,2,3, \ldots
$$

Demonstração. Por (A.2.10) para $k=1,2$, temos que

$$
r_{1}\left|1+a_{2}\right| \geq\left|a_{2}\right| \quad \text { e } \quad r_{2}\left|1+a_{2}+a_{3}\right| \geq\left|a_{3}\right|
$$

Portanto, $B_{2}=1+a_{2} \neq 0, B_{3}=1+a_{2}+a_{3} \neq 0$, e

$$
\left|\rho_{1}\right|=\left|\frac{a_{2}}{1+a_{2}}\right| \leq r_{1} \quad \text { e } \quad\left|\rho_{2}\right|=\left|\frac{a_{3}}{1+a_{2}+a_{3}}\right| \leq r_{2} .
$$

Usando indução, supomos agora que $B_{k+1} \neq 0$ e $\left|\rho_{k}\right| \leq r_{k}$ para $k=1,2,3, \ldots, m$, com $m \geq 2$, e os estabeleceremos para $k=m+1$. Mas teremos de distinguir dois casos, em acordo com o fato de $a_{m+2}=0$ ou $a_{m+2} \neq 0$. Se $a_{m+2}=0$, então, por uma das fórmulas de recorrência fundamentais $\left(\right.$ A.2.4) e do fato que em (A.2.9) $b_{k}=1$ para todo $k$,

$$
B_{m+2}=B_{m+1}+a_{m+2} B_{m}=B_{m+1}
$$

que é diferente de zero por hipótese; e

$$
\left|\rho_{m+1}\right|=\left|\frac{a_{m+2} B_{m}}{B_{m+2}}\right|=0 \leq r_{m+1} .
$$

Se, por outro lado, $a_{m+2} \neq 0$, então segue de (A.2.10), com $k=m+1$, que $r_{m+1}>0$. Além disso, por uma das fórmulas de recorrência fundamentais (A.2.4) obtemos

$$
B_{m+2}=\left(1+a_{m+1}+a_{m+2}\right) B_{m}-a_{m} a_{m+1} B_{m-2},
$$

de tal modo que, pela hipótese de indução e (A.2.10),

$$
\begin{aligned}
\left|\frac{B_{m+2}}{a_{m+2} B_{m}}\right| & =\left|\frac{1+a_{m+1}+a_{m+2}}{a_{m+2}}-\frac{a_{m+1}}{a_{m+2}} \frac{a_{m} B_{m-2}}{B_{m}}\right| \\
& \geq\left|\frac{1+a_{m+1}+a_{m+2}}{a_{m+2}}\right|-\left|\frac{a_{m+1}}{a_{m+2}}\right| r_{m-1} \geq \frac{1}{r_{m+1}}>0
\end{aligned}
$$


Portanto, $B_{m+2} \neq 0$ e $\left|\rho_{m+1}\right| \leq r_{m+1}$. Isto completa a indução e a demonstração deste teorema.

Pela fórmula determinante (A.2.6), a série infinita

$$
1+\sum_{k=1}^{\infty}\left(\frac{A_{k+1}}{B_{k+1}}-\frac{A_{k}}{B_{k}}\right)
$$

que é equivalente à fração continuada, pode ser escrita como

$$
1-\frac{a_{2}}{B_{1} B_{2}}+\frac{a_{2} a_{3}}{B_{2} B_{3}}-\frac{a_{2} a_{3} a_{4}}{B_{3} B_{4}}+\cdots
$$

que por sua vez é igual à série

$$
1+\sum_{k=1}^{\infty} \rho_{1} \rho_{2} \cdots \rho_{k}
$$

por (A.2.11). Quando então a fração continuada (A.2.9) satisfaz as desigualdades fundamentais (A.2.10), a série

$$
1+\sum_{k=1}^{\infty} r_{1} r_{2} \cdots r_{k}
$$

é um majorante para a série (A.2.19); isto é,

$$
\left|\frac{A_{k+1}}{B_{k+1}}-\frac{A_{k}}{B_{k}}\right|=\left|\rho_{1} \rho_{2} \cdots \rho_{k}\right| \leq r_{1} r_{2} \cdots r_{k}
$$

para $k=1,2,3, \ldots$. Isto é chamado por Wall de primeira interpretação das desigualdades fundamentais.

O Teorema de Worpitzky. Estamos agora em posição de enunciar e demonstrar o referido teorema: 
Teorema A.2.4 (Teorema de Worpitzky) Sejam $a_{2}, a_{3}, a_{4}, \ldots$ funções de quaisquer variáveis sobre um domínio $\mathcal{D}$ no qual

$$
\left|a_{k+1}\right| \leq \frac{1}{4}, \quad k=1,2,3, \ldots
$$

Então valem as seguintes afirmações:

(a) A fração continuada (A.2.9) converge uniformemente sobre $\mathcal{D}$.

(b) Os valores da fração continuada e de seus aproximantes estão no domínio circular

$$
\left|z-\frac{4}{3}\right| \leq \frac{2}{3}
$$

(c) A constante 1/4 é a "melhor" constante que pode ser usada em (A.2.22), e (A.2.23) é o "melhor" dominio de valores dos aproximantes.

Demonstração. (a) De (A.2.22) e da desigualdade $\left|1+\lambda_{1}+\lambda_{2}\right| \geq|1|-\left|\lambda_{1}\right|-\left|\lambda_{2}\right|$,

$$
\begin{aligned}
\frac{1}{3}\left|1+a_{2}\right| & \geq \frac{1}{3}\left(1-\frac{1}{4}\right)=\frac{1}{4} \geq\left|a_{2}\right|, \\
\frac{2}{4}\left|1+a_{2}+a_{3}\right| & \geq \frac{2}{4}\left(1-\frac{1}{4}-\frac{1}{4}\right)=\frac{1}{4} \geq\left|a_{3}\right|, \\
\frac{k}{k+2}\left|1+a_{k}+a_{k+1}\right| & \geq \frac{k}{2(k+2)} \\
& =\frac{k(k-2)}{(k+2) \frac{1}{4}}+\frac{1}{4} \\
& \geq \frac{k}{k+2} \frac{k-2}{k}\left|a_{k}\right|+\left|a_{k+1}\right|, \quad k=3,4, \ldots
\end{aligned}
$$

Conseqüentemente, a fração continuada satisfaz as desigualdades fundamentais (A.2.10) com

$$
r_{k}=\frac{k}{k+2}, \quad k=3,4,5, \ldots
$$


Assim sendo, a série majorante

$$
\begin{aligned}
1+\sum_{k=1}^{\infty} r_{2} r_{3} \cdots r_{k} & =1+\sum_{k=1}^{\infty}\left(\frac{1}{3} \cdot \frac{2}{4} \cdot \frac{3}{5} \cdot \frac{4}{6} \cdots \frac{k}{k+2}\right) \\
& =1+\sum_{k=1}^{\infty} \frac{2}{(k+1)(k+2)} \\
& =1+2 \sum_{k=1}^{\infty}\left(\frac{1}{k+1}-\frac{1}{k+2}\right)=2
\end{aligned}
$$

é convergente, e portanto concluímos que a fração continuada converge uniformemente para

$$
\left|a_{k+1}\right| \leq \frac{1}{4}
$$

$k=1,2,3, \ldots$, e que o módulo de seu valor não excede 2 . Considerando que $a_{k}$ pode ser igual a 0 , segue que os módulos dos valores dos aproximantes não excede 2.

(b) Agora, escrevemos a fração continuada na forma

$$
z=\frac{1}{1+\frac{1}{4} w} \quad \text { com } \quad w=\frac{x_{1}}{1+\frac{a_{3}}{1+\frac{a_{4}}{1+\ddots}}}
$$

com $\left|x_{1}\right| \leq 1$. É claro que $|w| \leq 2$. Qualquer aproximante da fração continuada pode, naturalmente, ser escrita na forma (A.2.30). Então

$$
|w|=4\left|\frac{z-1}{z}\right| \leq 2 \quad \text { ou } \quad\left|z-\frac{4}{3}\right| \leq \frac{2}{3}
$$

que é (A.2.23).

(c) Para vermos que 1/4 é a "melhor" constante que pode ser usada em (A.2.22), consideremos que $a_{k+1}=a$ para $k=1,2,3, \ldots$, com $a$ um número real; temos assim a fração continuada periódica 


$$
\frac{1}{1+\frac{a}{1+\frac{a}{1+\frac{a}{1+\ddots}}}}
$$

Considerando convenientemente as mudanças de variáveis $a=-u v$ e $1=u+v,(\mathrm{~A} .2 .32)$ passa a ser escrita

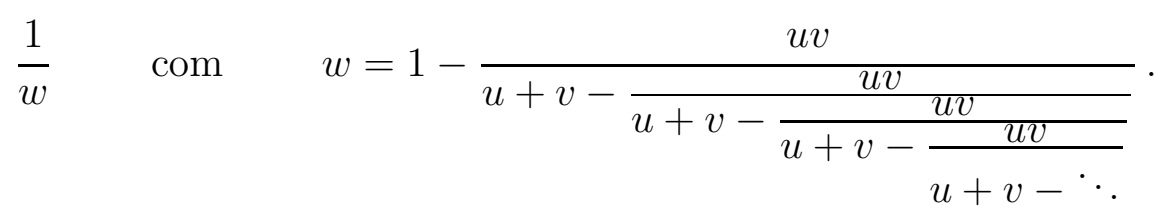

Para a fração continuada periódica $w$ não é muito difícil verificarmos que seu $k$-ésimo aproximante é dado por (vide [47], exercício 1.4 do Capítulo I)

$$
\frac{A_{k}}{B_{k}}=1-\frac{u v \sum_{j=0}^{k-1}\left(\frac{v}{u}\right)^{j}}{u+v \sum_{j=0}^{k-1}\left(\frac{v}{u}\right)^{j}}
$$

Para então sabermos o $k$-ésimo aproximante de (A.2.32), devemos escrever (A.2.34) na variável $a$. Como $v=1-u$, podemos obter $u$ como função de $a$ pela resolução da equação de segundo grau $u^{2}-u-a=0$. Denotando por $u_{1}$ e $u_{2}$ as soluções

$$
\frac{1 \pm \sqrt{1+4 a}}{2}
$$

vemos que o $k$-ésimo aproximante de (A.2.32) é igual a

$$
\frac{C_{k}}{D_{k}}=\frac{u_{1}+\left(1-u_{1}\right) \sum_{j=0}^{k-1}\left(\frac{1-u_{1}}{u_{1}}\right)^{j}}{u_{1}+\left(1-u_{1}\right)^{2} \sum_{j=0}^{k-1}\left(\frac{1-u_{1}}{u_{1}}\right)^{j}}
$$

Pela Definição A.2.1, uma fração continuada é convergente se o limite 


$$
\lim _{k \rightarrow \infty} \frac{C_{k}}{D_{k}}
$$

existe e é finito. Dessa condição conclui-se que a fração continuada periódica (A.2.32) é convergente se $a \geq-1 / 4$.

Para mostrar que (A.2.23) é o "melhor" domínio, basta que se note que os valores da fração continuada

$$
z=\frac{1}{1+\frac{a_{2}}{1-\frac{1 / 4}{1-\frac{1 / 4}{1-\ddots}}}}=\frac{1}{1+2 a_{2}}
$$

preenchem o domínio (A.2.23) conforme $a_{2}$ varia sobre o domínio $\left|a_{2}\right| \leq 1 / 4$. 



\section{Referências Bibliográficas}

[1] W. Balser. "From Divergent Power Series to Analytic Functions", Lecture Notes in Mathematics 1582, Springer, Germany (1994).

[2] W. Balser. "Formal Power Series and Linear Systems of Meromorphic Ordinary Differential Equations", Springer-Verlag, New York (2000).

[3] W. Balser e V. Kostov. "Singular Perturbation of Linear Systems with a Regular Singularity", J. Dyn. Control Syst. 8, 313-322 (2002).

[4] W. Balser e V. Kostov. "Recent Progress in the Theory of Formal Solutions for ODE and PDE", Appl. Math. Comput. 141, 113-123 (2003).

[5] Silas L. de Carvalho. "Persistência de Ordem em Modelos Ferromagnéticos na Presença de Campos Auto-Similares Quase-Aleatórios", Dissertação de Mestrado IFUSP (2007).

[6] E. A. Coddington e N. Levinson. "Theory of Ordinary Differential Equations", Krieger (1984).

[7] William R. P. Conti. "Teorema Central do Limite para o Modelo $O(N)$ de Heisenberg Hierárquico na Criticalidade e o Papel do Limite $N \rightarrow \infty$ na Dinâmica dos Zeros de Lee-Yang", Dissertação de Mestrado - IFUSP (2008).

[8] William R. P. Conti e Domingos H. U. Marchetti. "Hierarchical Spherical Model as a Viscosity Limit of Corresponding $O(N)$ Model", arXiv:0804.2704, v.1 (2008).

[9] William R. P. Conti e Domingos H. U. Marchetti. "The Inverse Function Theorem of $s$-Summable Gevrey Asymptotic Functions of a Different Variable", artigo em preparação. 
[10] R. Courant e D. Hilbert. "Methods of Mathematical Physics", Vol. 1, John Wiley \& Sons (1989).

[11] Joël De Coninck. "Infinitely Divisible Distribution Functions of Class $L$ and the Lee-Yang Theorem", Commun. Math. Phys. 96, 373-385 (1984).

[12] W. F. Donoghue Jr. . "Monotone Matrix Function and Analytic Continuation", Die Grundlehren der mathematischen Wissenschaften 207 (1974).

[13] Peter L. Duren. "Univalent Functions", Die Grundlehren der mathematischen Wissenschaften 259 (1983).

[14] Freeman J. Dyson. "Existence of a Phase-Transition in a One-Dimensional Ising Ferromagnet", Comm. Math. Phys. 12, 91-107 (1969).

[15] Freeman J. Dyson. "An Ising Ferromagnet with Discontinuous Long-Range Order" Comm. Math. Phys. 21, 269-283 (1971).

[16] Lawrence C. Evans. "Partial Differential Equations", Graduated Studies in Mathematics, Vol. 19, AMS (1998).

[17] Giovanni Felder. "Renormalization Group in the Local Potential Approximation", Comm. Math. Phys. 111, 101-121 (1987).

[18] M. S. Floater e T. Lyche. "Two Chain Rules for Divided Differences and Faà di Bruno's Formula", Mathematics of Computation 76, 867-877 (2007).

[19] G. B. Folland. "Introduction to Partial Differential Equations", Mathematical Notes, Princeton University Press (1976).

[20] J. Fröhlich, R. Israel, E. H. Lieb e B. Simon. "Phase Transitions and Reflection Positivity: I. General Theory and Long Range Lattice Models", Commun. Math. Phys. 62, 1-34 (1978).

[21] J. Fröhlich, A. Mardin e V. Rivasseau. "Borel Summability of the $1 / N$ Expansion for the $N$-Vector $[O(N)$ Non-Linear $\sigma]$ Models", Commun. Math. Phys. 86, 87-110 (1982).

[22] G. Gallavotti. "Some Aspects of the Renormalization Problems in Statistical Mechanics and Field Theory", Memorie dell'Accademia dei Lincei, S. 8, Vol. 15 (1978). 
[23] Leonardo F. Guidi e Domingos H. U. Marchetti. "Renormalization Group Flow of the Two-Dimensional Hierarchical Coulomb Gas", Commun. Math. Phys. 219, 671-702 (2001).

[24] Laurence S. Hall. "Bessel Function $J_{\nu}(z)$ of Complex Order and Its Zeros", Proc. Camb. Phil. Soc. 63, 141-146 (1967).

[25] Takashi Hara, Tetsuya Hattori e Hiroshi Watanabe. "Triviality of Hierarchical Ising Model in Four Dimensions", Commun. Math. Phys. 220, 13-40 (2001).

[26] Einar Hille. "Analytic Function Theory", Vol. 1, Chelsea, second edition (1982).

[27] L. Iliev. "Laguerre Entire Functions", Bulgarian Academy of Sciences, Sofia (1987).

[28] Mark Kac e Colin J. Thompson. "Spherical Model and Infinite Spin Dimensionality Limit”, Phys. Norvegica, Vol. 5, 163-168 (1971).

[29] Yuri V. Kozitsky. "Hierarchical Model of a Ferromagnet. Self-Similar Block-Spin Distributions and the Lee-Yang Theorem", Rep. Math. Phys. 26, 429-445 (1988).

[30] Yuri V. Kozitsky. "Hierarchical Ferromagnetic Vector Spin Model Possessing the Lee-Yang Property. Thermodynamic Limit at the Critical Point and Above", Journ. Stat. Phys. 87, 799-820 (1997).

[31] Yuri V. Kozitsky e Lech Wołowiski. "Laguerre Entire functions and Related Locally Convex Spaces", Los Alamos Eletronic Preprint arXiv:math.CV/9812111, v.1 (1998).

[32] Yuri V. Kozitsky e Lech Wołowiski. "A Nonlinear Dynamical System on the Set of Laguerre Entire Functions", Elsevier Preprint (2001).

[33] Erwin Kreyszig e John Todd. "The Radius of Univalence of Bessel Functions I", Illinois J. Math. 4, 143-149 (1960).

[34] H. Kunz e G. Zumbach. "Phase Transition in a Nematic N-Vector Model: The Large N Limit", J. Phys. A25, 6155-6162 (1992).

[35] Antti J. Kupiainen. "On the 1/n Expansion", Commun. Math. Phys. 73, 273-294 (1980). 
[36] B. J. Levin. "Distribution of Zeros of Entire Functions", American Mathematical Society (1964).

[37] Paulo C. Lima. "Renormalization Group Fixed Points in the Local Potential Approximation for $d \geq 3$ ", Commun. Math. Phys. 170, 529-539 (1995).

[38] J. E. Littlewood. "Lectures on the Theory of Functions", Oxford University Press (1944).

[39] D. A. Lutz, M. Miyake e R. Schäfke. "On the Borel Summability of Divergent Solutions of the Heat Equation", Nagoya Math. J. 154, 1-29 (1999).

[40] T. D. Lee e C. N. Yang. "Statistical Theory of Equation of States and Phase Transitions. II Lattice Gas and Ising Model", Phys. Rev. 87, 404-409 (1952).

[41] Domingos H. U. Marchetti, William R. P. Conti e Leonardo F. Guidi. "Hierarchical Spherical Model from a Geometrical Point of View", Journ. Stat. Phys. 132, 811838 (2008).

[42] Domingos H. U. Marchetti e William R. P. Conti. "Global Formal Series Solution of a Singularly Perturbed First Order Partial Differential Equation Coming from a Problem in Statistical Mechanics", artigo em preparação.

[43] Charles M. Newman. "Zeros of the Partition Function for Generalized Ising Systems", Commun. Pure Appl. Math. XXVII, 143-159 (1974).

[44] Charles M. Newman. "Inequalities for Ising Models and Field Theories wich Obey the Lee-Yang Theorem", Commun. Math. Phys. 41, 1-9 (1975).

[45] V. V. Petrov. "Sums of Independent Random Variables", Berlin, Heidelberg, New York: Springer Verlag (1975).

[46] C. K. Qu e R. Wong. ""Best Possible" Upper and Lower Bounds for the Zeros of the Bessel Function $J_{\nu}(x)$ ", Trans. Amer. Math. Soc. 351, 2833-2859 (1999).

[47] H. S. Wall. "Analytic Theory of Continued Fractions", D. Van Nostrand Co. (1948).

[48] G. N. Watson. "A Treatise on the Theory of Bessel Functions", Macmillan, New York (1944). 
[49] Hiroshi Watanabe. "Triviality of Hierarchical $O(N)$ Spin Model in Four Dimensions with Large N", Journ. Stat. Phys. 115, 1669-1713 (2004).

[50] Takesi Yamanaka. "A New Higher Order Chain Rule and Gevrey Class", Ann. Global Anal. Geom. 7, 179-203 (1989).

[51] Gil Zumbach. "The Renormalization Group in the Local Potential Approximation and Its Applications to the $O(n)$ model", Nucl. Phys. B413, 754-770 (1994). 